\title{
DEVELOPMENT OF A COMPOSITE INTRAMEDULLARY NAIL FOR TREATING FEMORAL SHAFT FRACTURES
}

\author{
by \\ Saeid Samiezadeh \\ Bachelor of Science (Mechanical Engineering- Solid Mechanics) \\ Semnan University, Semnan, Iran, 2008 \\ Master of Science (Mechanical Engineering- Applied Design) \\ Tarbiat Modares University, Tehran, Iran, 2010
}

\author{
A dissertation \\ presented to Ryerson University \\ in partial fulfillment of the requirements for \\ the degree of Doctor of Philosophy \\ in the program of Mechanical Engineering
}

Toronto, Ontario, Canada, 2016

(C) Saeid Samiezadeh, 2016 


\section{Author's Declaration for Electronic Submission of a Dissertation}

I hereby declare that I am the sole author of this dissertation. This is a true copy of the dissertation, including any required final revisions, as accepted by my examiners.

I authorize Ryerson University to lend this dissertation to other institutions or individuals for the purpose of scholarly research.

I further authorize Ryerson University to reproduce this dissertation by photocopying or by other means, in total or in part, at the request of other institutions or individuals for the purpose of scholarly research.

I understand that my dissertation may be made electronically available to the public. 


\author{
Abstract \\ Development of a Composite Intramedullary Nail for Treating Femoral Shaft Fractures \\ Saeid Samiezadeh \\ Doctor of Philosophy \\ Mechanical and Industrial Engineering \\ Ryerson University \\ 2016
}

Intramedullary nails are the primary choice for treating long bone fractures. However, the high axial rigidity of conventional nails, can significantly reduce compression at the fracture site, and thereby inhibit bone healing. It can also lead to subsequent bone loss upon fracture healing. Fibrereinforced composites have been suggested as an alternative material of choice in the design of fracture fixation implants to address these drawbacks. There are very few studies in the literature on the use of composite materials for intramedullary nails. In particular, there are no known studies which have considered the optimization of such implants to fulfill the requirements of a proper fracture healing. The purpose of the current thesis is to develop a composite intramedullary nail made of carbon-fibre/epoxy whose structure is optimized to provide a preferred mechanical environment for fracture healing. The thickness and stacking sequence of the composite tube were optimized using closed-form expressions for structural rigidities of a composite tube to minimize axial rigidity of the structure, while minimally sacrificing the bending and torsional rigidities. The actual performance of the best nail candidates inside the human femur was then examined in an experimentally validated finite element model.

It was found that a composite nail with an outer diameter of $14 \mathrm{~mm}$ and a stacking sequence of $\left[00_{2} /-45 / 45_{2} /-45 / 0 /-45 / 45_{2} /-45_{2} / 45_{2} /-45 / 90_{2}\right]$ showed an overall superiority compared to the other configurations. The expressions for rigidity yielded an axial rigidity of $3.7 \mathrm{MN}$, and bending and torsional rigidities of 70.3 and $70.9 \mathrm{~N} . \mathrm{m}^{2}$, respectively, which correlated well with the results of mechanical testing on the manufactured specimens (i.e. $3.74 \pm 0.05 M N, 66.9 \pm 1.0$ $N . m^{2}$, and $70.7 \pm 2.0 N . m^{2}$ for axial, bending, and torsional rigidities, respectively). The

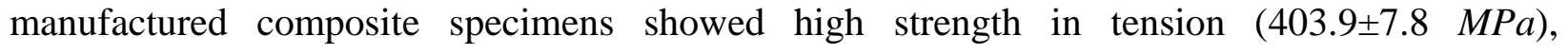
compression (316.9 $\pm 10.9 \mathrm{MPa})$, bending $(405.3 \pm 8.1 \mathrm{MPa})$, and torsion $(328.5 \pm 7.3 \mathrm{MPa})$. 
Moreover, a fatigue limit of $27 \mathrm{kN}$ was obtained for the composite nail. The stiffness of the composite nail was found to remain almost constant versus the number of cycles. Overall, the findings of this thesis suggest that the proposed intramedullary nail is a potential candidate for use as an alternative to the conventional intramedullary nails. 


\section{Acknowledgement}

I would like to express my special appreciation and thanks to my supervisors Dr. Habiba Bougherara and Dr. Zouheir Fawaz, you have been a tremendous mentor for me. Your advice on both research as well as on my career have been invaluable. Without your encouragement, support, and guidance, I never would have completed my PhD.

I would like to also thank the program coordinator, Dr. Ahmad Ghasempoor, for his guidance and support during my studies at Ryerson University.

Lastly, I recognize my beloved wife, for her unconditional support in good and bad times. Words cannot express how grateful I am to you for all of the sacrifices that you have made on my behalf. 


\section{Table of Contents}

Author's Declaration for Electronic Submission of a Dissertation.......................................... ii

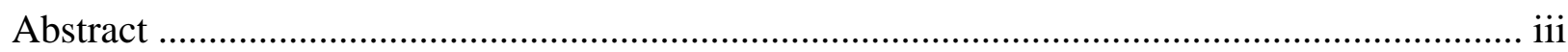

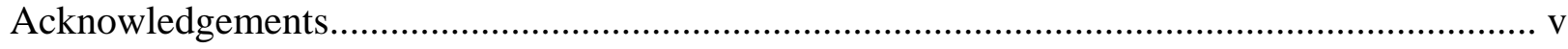

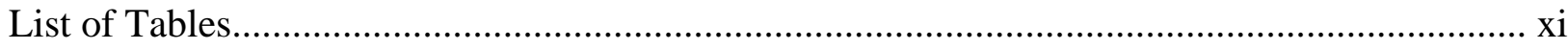

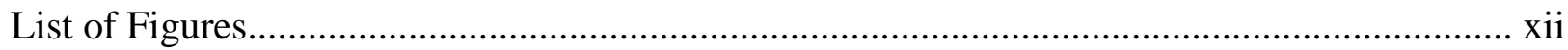

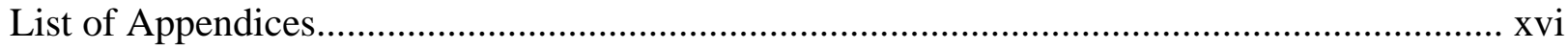

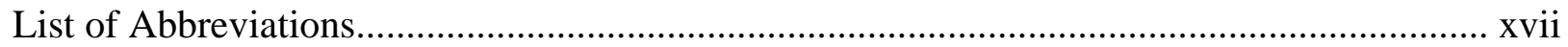

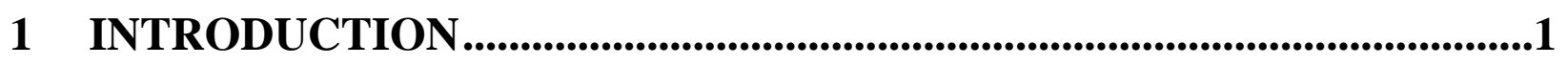

1.1 Background motivation............................................................................... 1

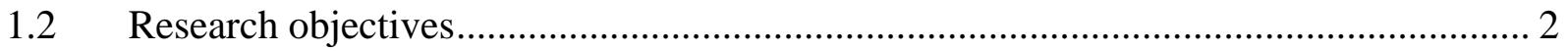

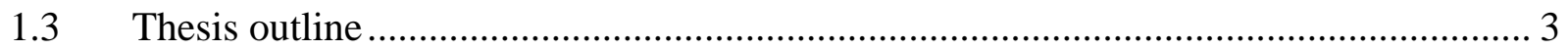

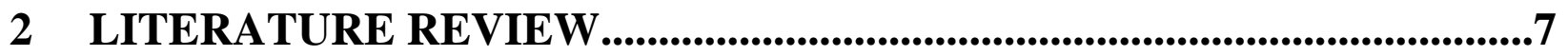

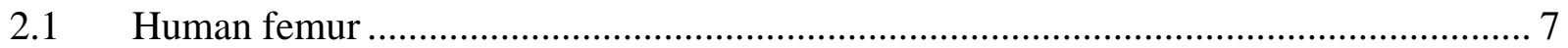

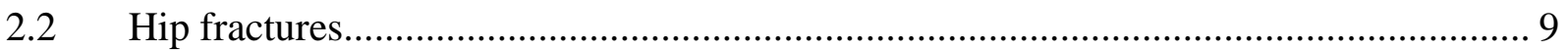

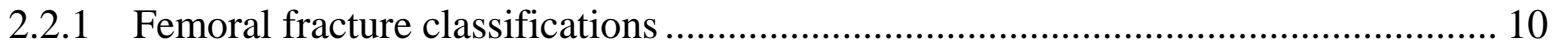

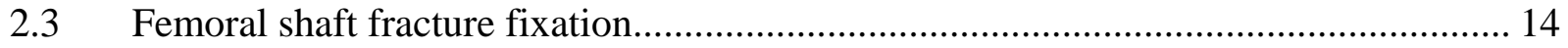

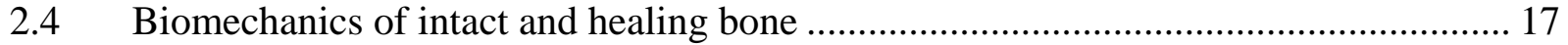

2.5 The major limitations of conventional IM nails ................................................. 24

2.6 Previous attempts to solve the problem of stress shielding ................................... 24

2.6.1 Previous studies on composite fracture fixation devices ................................... 25

2.7 Shortcomings of previous biomechanical studies on IM nailing ........................... 28

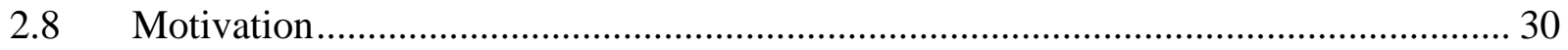

\section{OPTIMIZATION OF A COMPOSITE BONE PLATE USING THE} SELECTIVE STRESS SHIELDING APPROACH ............................................31 


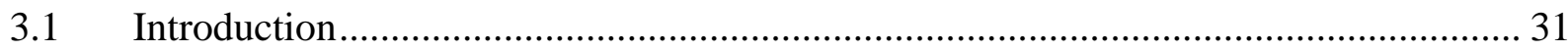

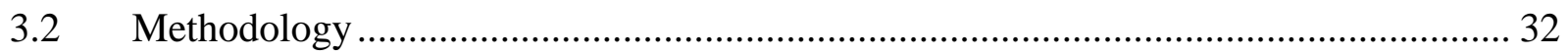

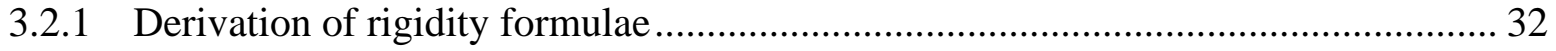

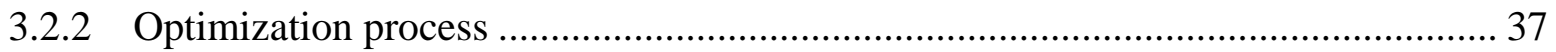

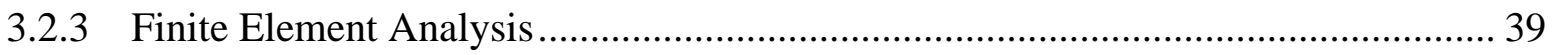

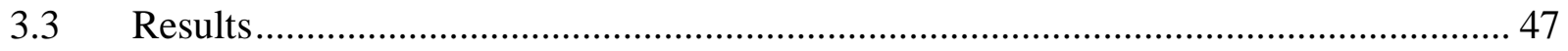

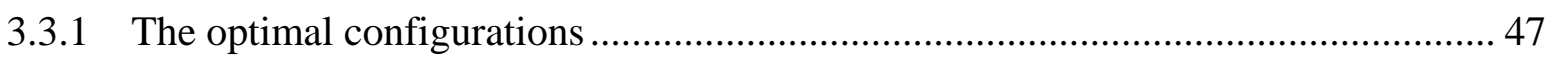

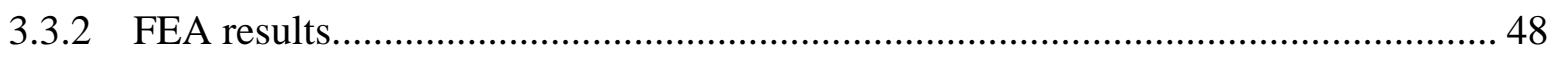

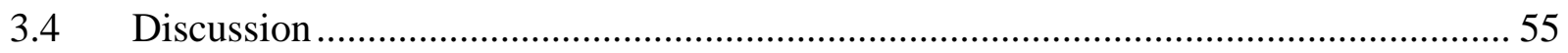

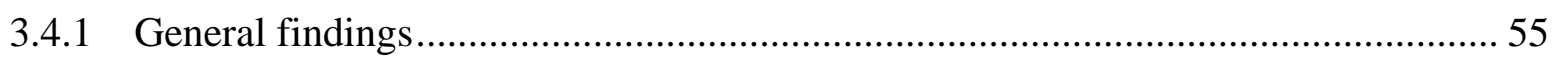

3.4.2 Selection of the best configuration …………................................................... 56

3.4.3 Comparison of the best composite configuration with conventional Ti plates..........57

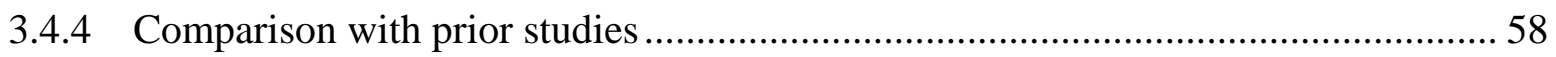

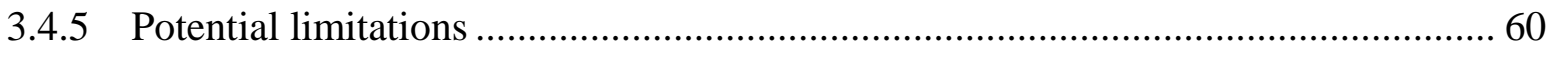

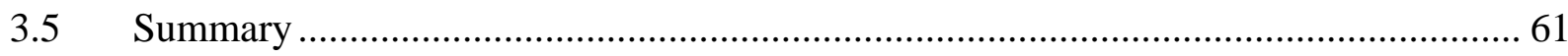

\section{STRUCTURAL RIGIDITIES OF A COMPOSITE TUBE .....................63}

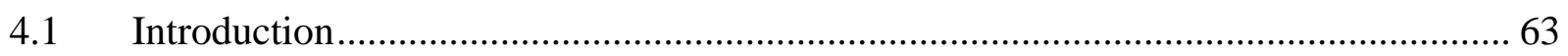

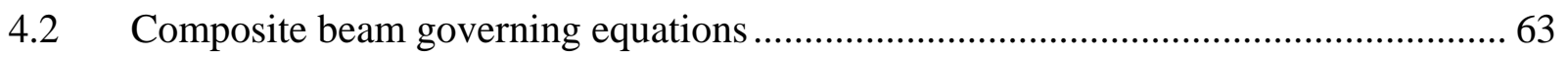

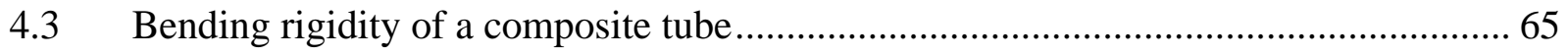

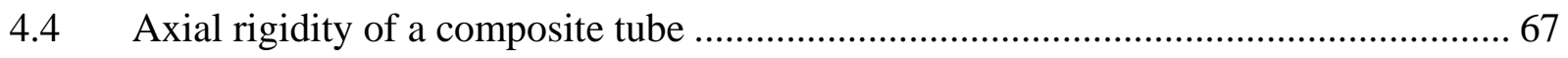

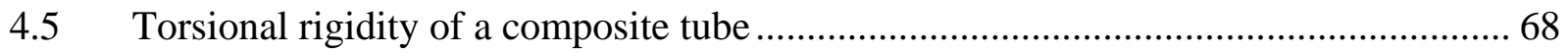

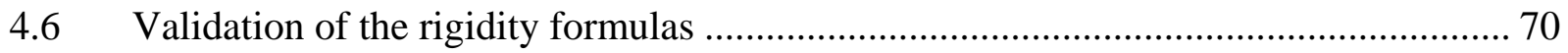

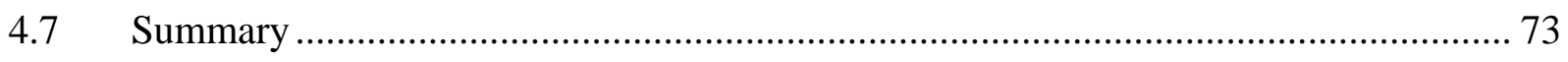

5 STRUCTURAL COUPLINGS IN A COMPOSITE TUBE .......................75

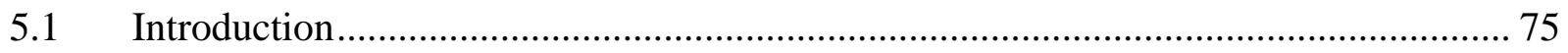




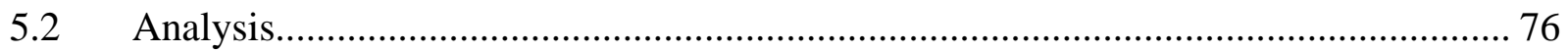

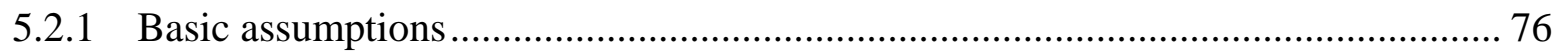

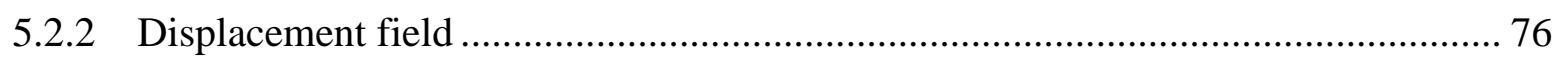

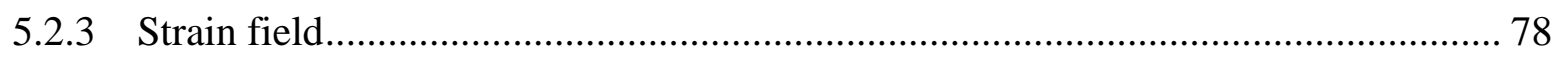

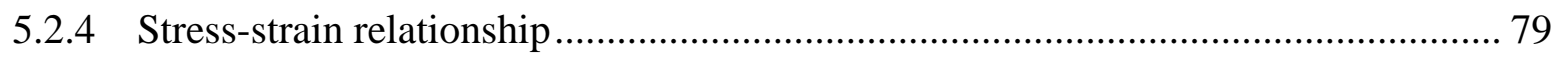

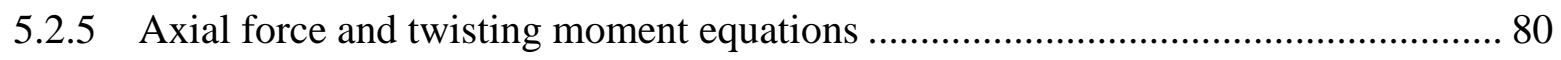

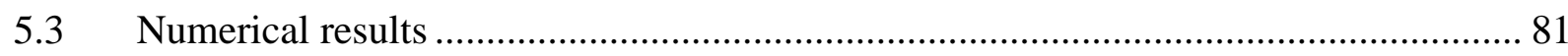

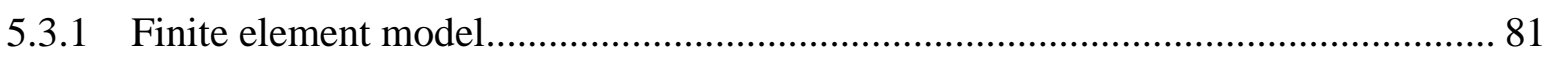

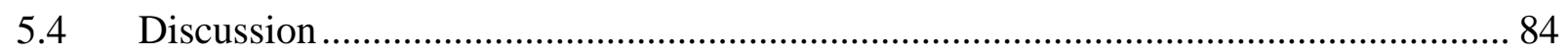

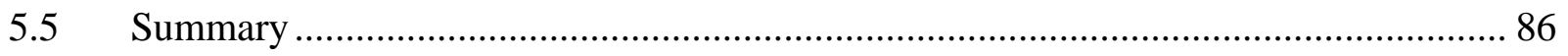

\section{OPTIMIZATION OF THE COMPOSITE IM NAIL STRUCTURE .......88}

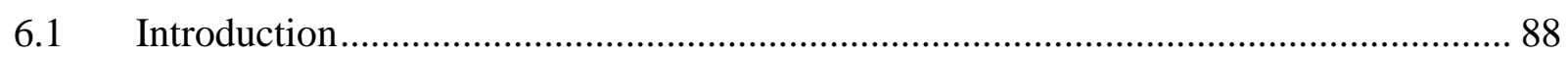

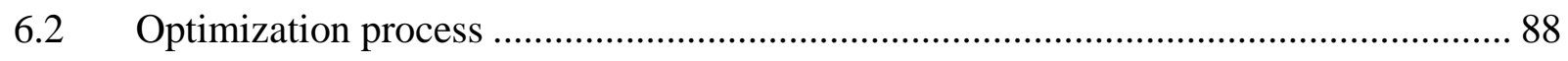

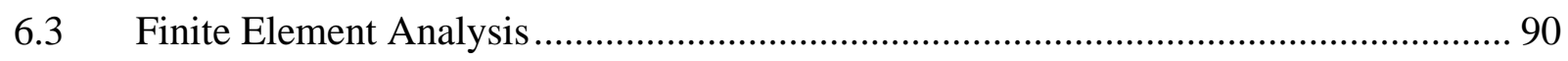

6.3.1 Mechano-biochemical (thermodynamic-based) model.............................................. 93

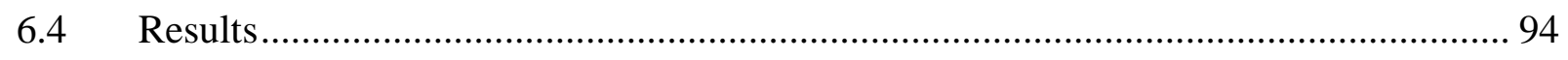

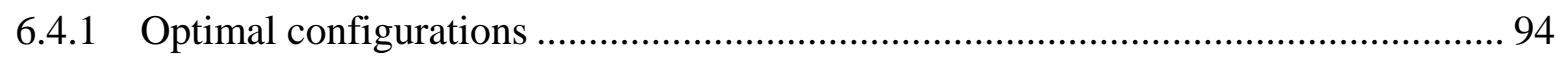

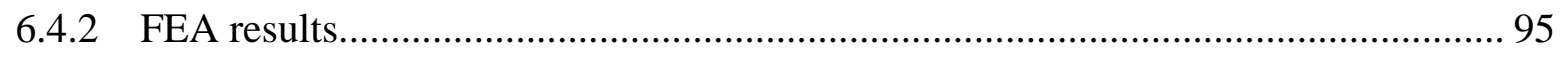

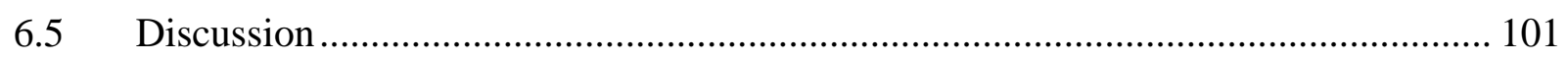

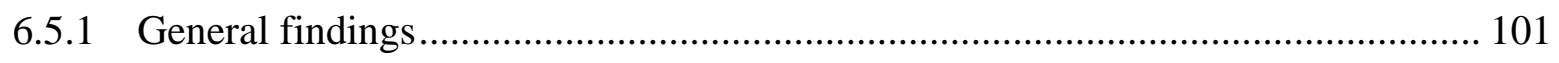

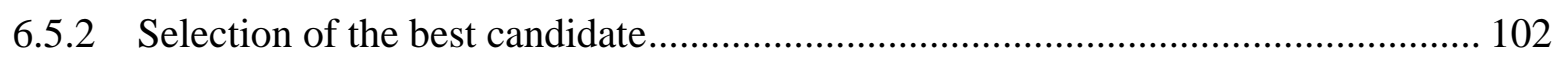

6.5.3 Comparison of the best composite candidate with a conventional Ti-alloy nail .... 102

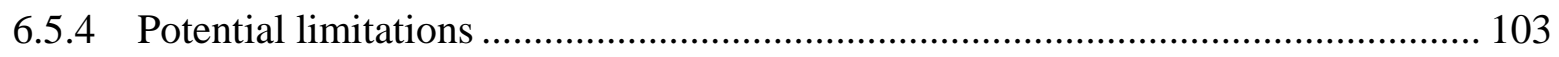

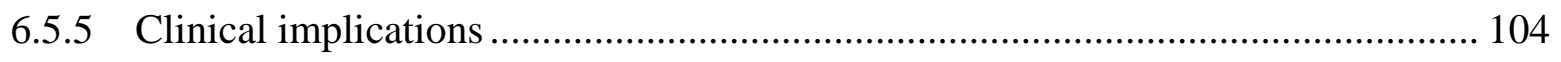

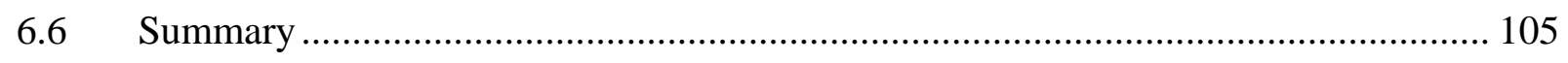




\section{BIOMECHANICAL TESTING OF CARBON-FIBRE/EPOXY COMPOSITE IM NAIL .........................................................................................106}

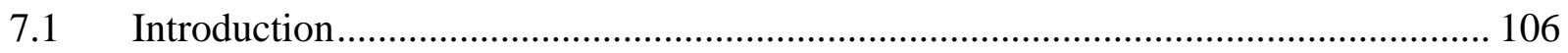

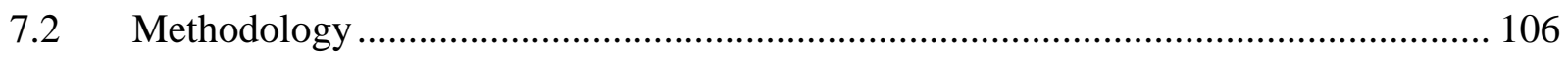

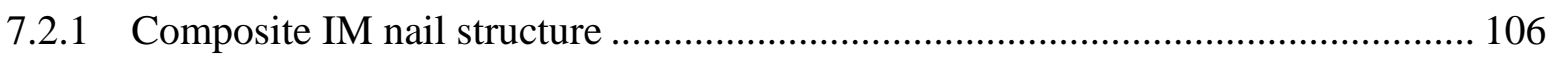

7.2.2 Void fraction measurement ................................................................................ 107

7.2.3 Moisture absorption tests ................................................................................ 107

7.2.4 Compression tests ...................................................................................... 108

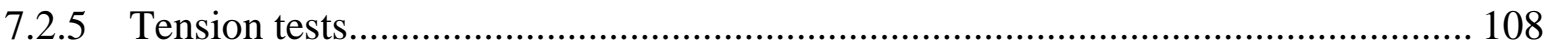

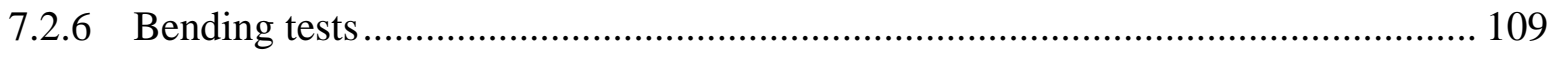

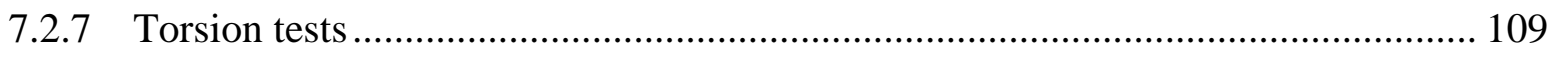

7.2.8 Rockwell hardness tests ............................................................................. 110

7.2.9 Surface roughness measurements …………….............................................. 110

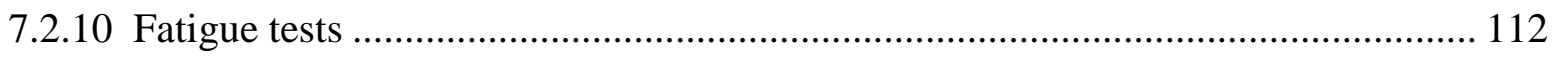

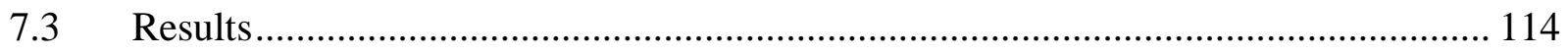

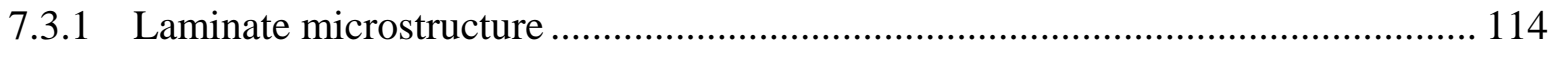

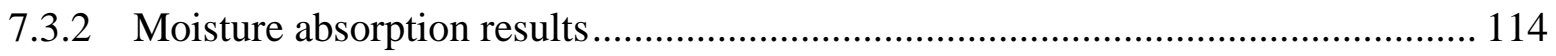

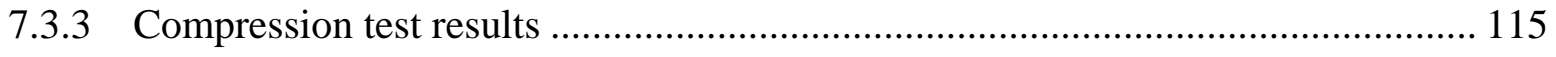

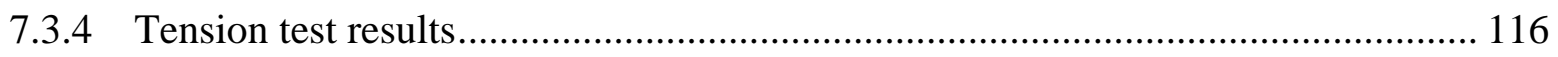

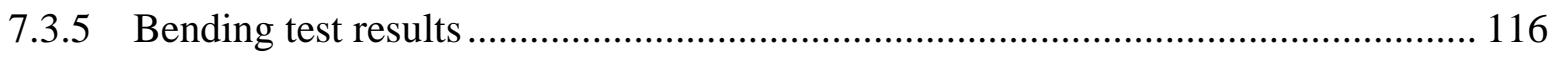

7.3.6 Elastic moduli comparison................................................................................ 117

7.3.7 Torsion test results ....................................................................................... 117

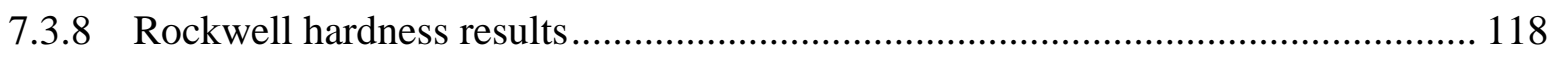

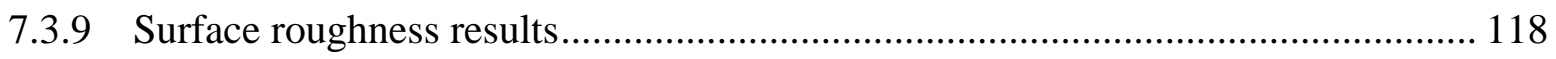

7.3.10 Fatigue test results...................................................................................... 119 


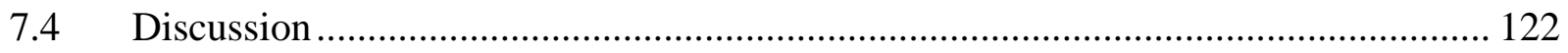

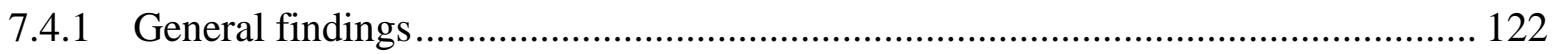

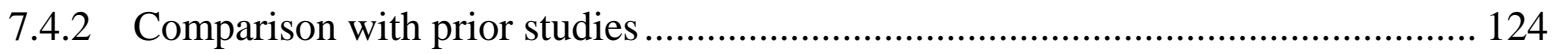

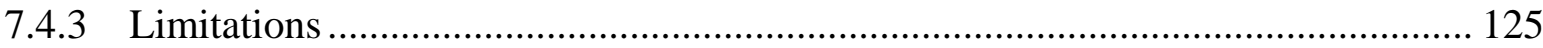

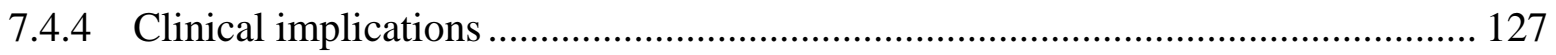

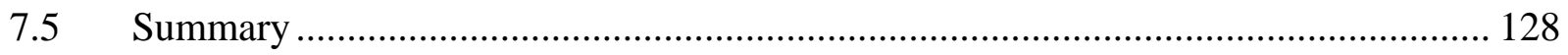

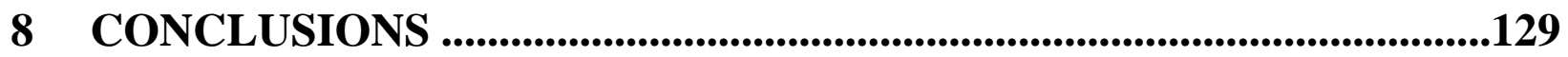

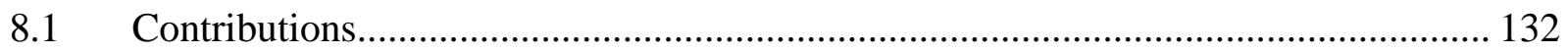

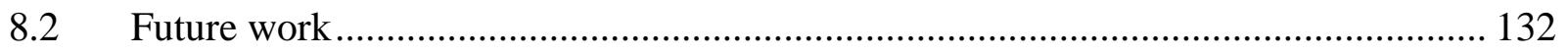

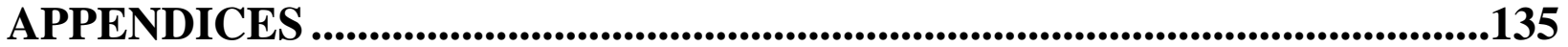

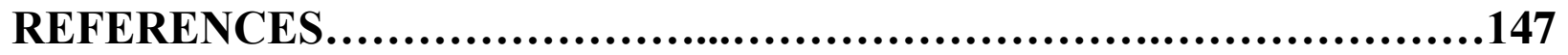




\section{List of Tables}

Table 2-1. Winquist and Hansen classification of fracture comminution ............................... 11

Table 3-1. Linear elastic material properties used in this study [104-109] ............................. 43

Table 3-2. Force components for muscle and joint reaction forces..................................... 44

Table 3-3. Optimal configurations in the intervals of bending rigidity. EA: Axial rigidity, EI:

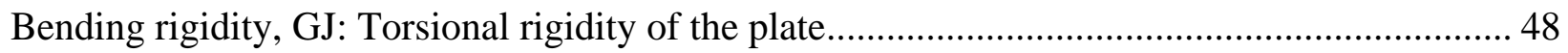

Table 4-1. Different lay-up configurations used for validation of the rigidity formulas ............. 74

Table 5-1. Linear elastic material properties of prepreg CF/epoxy plies ............................... 82

Table 5-2. Different lay-ups used in the current simulations, their corresponding $a_{12}$ coefficient, and their axial displacements following application of a 100 N.m twisting moment. ............... 84

Table 6-1. Linear elastic material properties used in this chapter [107-109] .......................... 91

Table 6-2. Optimal configurations in the intervals of bending rigidity .................................. 95

Table 6-3. Results showing axial stiffness and microstrain values at different locations of the

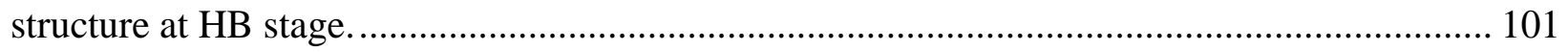

Table 7-1. Mechanical properties of Ti-alloy, the current CF/epoxy and some previously developed composite IM nails 125 


\section{List of Figures}

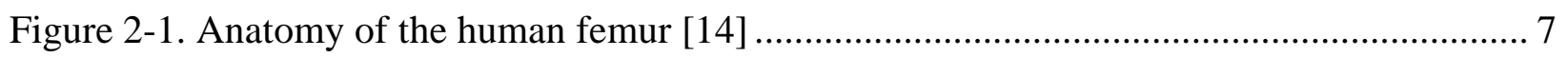

Figure 2-2. The main axes in human femur....................................................................... 8

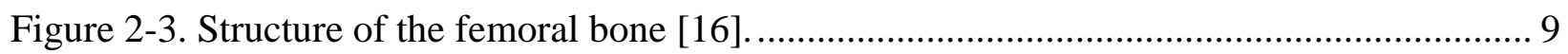

Figure 2-4. The Winquist-Hansen classification for femoral shaft fractures [2] ......................... 11

Figure 2-5. OTA classification of femoral shaft fractures [27] ................................................... 13

Figure 2-6. Femoral plating for a simple fracture pattern [2] ………………………………... 15

Figure 2-7. IM nailing for a simple fracture pattern [33 ......................................................... 16

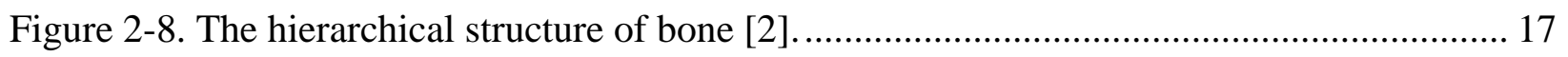

Figure 2-9. Elastic modulus of bone samples tested in tension after exposure to different

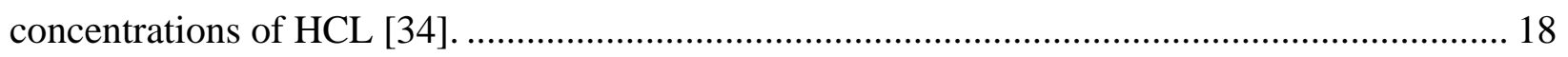

Figure 2-10. Effects of collagen fiber direction on the resistance to loads applied in different

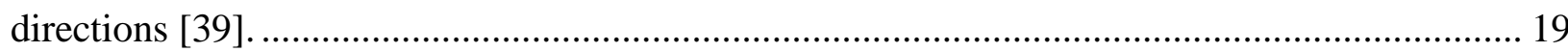

Figure 2-11. The relationship of trabecular bone density to compressive strength and modulus. 19 Figure 2-12. A comparison of the moments of inertia and resulting strengths when fracture callus is located A) on the outer surface, B) on the bone surfaces, or C) in the medullary canal [42]... 20 Figure 2-13. Changes in the cross-sectional area of a healing femoral fracture in rats [43] ....... 20 Figure 2-14. Torque versus angular displacement plots taken from experimental long bones at different stages of healing in rabbits. The numbers in the graph show time in days [44]............ 21 Figure 2-15. The effect on bone mineral content of different cyclic displacements applied daily within a healing fracture (upper curve, $0.5 \mathrm{~mm}$; middle curve, $1.0 \mathrm{~mm}$; lower curve, $2.0 \mathrm{~mm}$ for

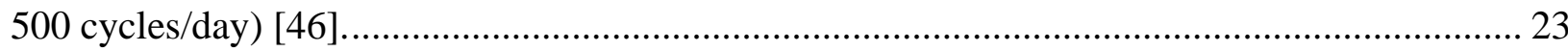

Figure 3-1. Rectangular laminated beam subjected to (a) axial load and bending moment, (b)

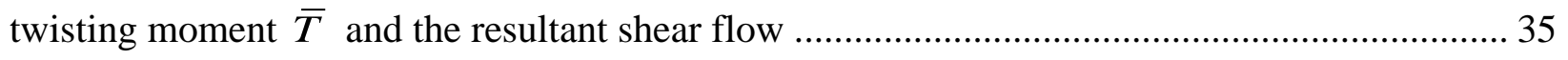

Figure 3-2. Finite element models of a femur with a transverse (a), proximal medial to distal lateral (PMDL) oblique (b), and proximal lateral to distal medial (PLDM) oblique (c) Vancouver B1 periprosthetic fracture, which was fixed with an eight-hole bone plate. 
Figure 3-3. Diagram showing the five major muscles and hip joint forces applied to the femur. 44

Figure 3-4. Schematic figure showing axial and shear movement measurements at the fracture site

Figure 3-5. Axial (a) and shear movement (b) between fracture fragments in transverse and oblique Vancouver B1 fracture fixed with different composite bone plate configurations (C1-C14) as well as the metallic plate (C15). 49

Figure 3-6. The inverse safety factor (ISF) for composite bone plate configurations (C1-C14) (based on Tsai-Hill failure criterion) and the metallic bone plate $(\mathrm{C} 15)$ (based on maximum equivalent stress failure criterion) for transverse and oblique Vancouver B1 fractures (a). The distribution of the ISF in a typical composite bone plate configuration (C7) for transverse (b), PMDL oblique (c), and PLDM oblique (d) fractures. 50

Figure 3-7. Compressive normal force (a) and contact bending moment (b) at a transverse and oblique Vancouver B1 fracture site fixed with composite (C1-C14) and metallic $(\mathrm{C} 15)$ bone plates.

Figure 3-8. Mean nodal von Mises stress and the maximum deformation in the intact femur, the healed femur fixed with the composite plate configurations (C1-C14), and the healed femur fixed

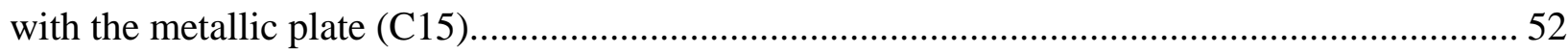

Figure 3-9. Strain distribution in the intact femur (a), the healed femur implanted with CF/epoxy plate (b) and the healed femur implanted with Ti plate (c)..... 53

Figure 3-10.Validation of FE results against IR thermography camera measurements by Bagheri et al. [91]. Values show the sum of principal stresses on the bone surface. (a) and (d) show FE results in healed and fractured femur respectively, (b) and (e) shows IR thermography camera measurements in aforementioned configurations (adopted with permission), (c) and (f) illustrate the graphs with slope and $\mathrm{R}^{2}$. The values in blue show the percentage error for each point....... 54

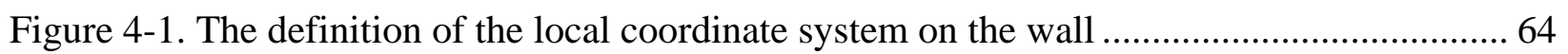
Figure 4-2. The standard testing setup for the four-point bending tests used in the simulations . 71 Figure 4-3. Axial rigidity values obtained from closed-form expression versus those obtained from 3D FEA 72 
Figure 4-4. Bending rigidity values obtained from 3D FEA versus those obtained from closedform expression.....

Figure 4-5.Torsional rigidity values obtained from 3D FEA versus those obtained from closedform expression. 73

Figure 5-1. The definition of the local coordinate system on the wall and the global coordinate system at the centre of the composite beam cross-section..... 77

Figure 5-2. Cylindrical composite tube in which each ply is meshed with 8-noded solid elements.

Figure 5-3. Axial deformation of the nodes located on a typical path on the beam wall as a result of applied twisting moment of 100 N.m. 83

Figure 6-1. Finite element models of a femur with a transverse (a), proximal medial to distal lateral (PMDL) oblique (b), and proximal lateral to distal medial (PLDM) oblique (c) fracture, which was fixed with an IM nail. 93

Figure 6-2. Fracture opening (a) and shear movement (b) at the fracture site for transverse and oblique mid-shaft fractures with the use of composite (C1-C7) and conventional metallic (C8) IM nails. 96

Figure 6-3. Nail inverse safety factor (ISF) for composite IM nail candidates (C1-C7) and the metallic IM nail (C8) in transverse and oblique mid-shaft femoral fractures...... 97

Figure 6-4. Compressive normal force at fracture (a) and average nodal stress in the bone at the vicinity of the fracture (b) for composite IM nail candidates (C1-C7) and the metallic IM nail (C8) in transverse and oblique mid-shaft femoral fractures 98

Figure 6-5. Long term average bone loss in the femoral shaft fixed with the composite IM nail candidates (C1-C7) and metallic IM nail (C8) (a). Percent change in femoral density in response to a typical IM nail candidate (b) as well as the metallic IM nail (c). 99 Figure 6-6. Experimental setup used by Bougherara et al. [56] to validate FE results. 100 Figure 7-1. (a) A typical microscopic image of a specimen's cross-section used to calculate the porosity. (b) Estimated black porous areas of the cross-section in ImageJ software. 107 
Figure 7-2. Quasi-static mechanical tests set up: (a) compression test (b) bending test (c) tension test (d) torsion test (e) Rockwell Hardness test.

Figure 7-3. Experimental setup for fatigue testing using IR camera

Figure 7-4. Schematic graph showing nonlinear response due to changing modulus and permanent inelastic strains [156]. 114

Figure 7-5. Moisture absorption results showing the weight gain of the composite tubes. 115

Figure 7-6. (a) Load-displacement diagram showing compression test results. (b) A typical specimen failed in compression and cut in half. The buckling of the outermost plies is evident.

Figure 7-7. (a) Tension test results for the specimens. (b) A typical specimen failed in tension test. 116

Figure 7-8. (a) 3-point bending test results for the specimens. (b) A typical specimen failed in the bending test. The specimen failed due to fibre breakage in the tension side and local buckling in the compression side. 117

Figure 7-9. Torsion test results for the specimens and a typical specimen failed in the torsion test. Delamination between the 0 -direction plies and the adjacent plies is evident. 118

Figure 7-10. Variation of surface temperature versus number of cycles for different stress levels in a typical specimen.

Figure 7-11. Thermographic temperature distribution contour following temperature stabilization at different stress level. UTS: ultimate tensile strength 120

Figure 7-12. Thermographic results showing the variation of maximum stabilized temperature versus the maximum applied stress. The bilinear behavior of the data is evident in the figure. 121 Figure 7-13. Static modulus versus number of cycles for a typical composite specimen 121 Figure 7-14. Dynamic modulus versus number of cycles for a typical composite specimen..... 122 


\section{List of Appendices}

A 1. MATLAB code used to calculate the structural rigidities of composite bone plates ......... 135

A 2. MATLAB code used to calculate the structural rigidities of composite IM nails ............. 139 


\section{List of Abbreviations}

\begin{tabular}{ll} 
AFP & Advanced fiber placement \\
ASTM & American society for testing and materials \\
BisGMA & Bisphenol A dimethacrylate \\
BMD & Bone mineral density \\
CAD & Computer-aided design \\
CAS & Circumferential asymmetric stiffness \\
CF & Carbon fibre \\
CUS & Circumferential uniform stiffness \\
DEXA & Dual-energy X-ray absorptiometry \\
FEA & Finite element analysis \\
HB & Healed bone \\
HCFS & High cycle fatigue strength \\
IM & Intramedullary \\
IPO & Immediate-post operative \\
IR & Infrared \\
IRT & Infrared thermography \\
ISF & Inverse safety factor \\
MRI & Magnetic resonance imaging \\
OTA & Orthopaedic trauma association \\
PEEK & Polyether ether ketone \\
PLDM & Proximal lateral to distal medial \\
PMDL & Post-operative \\
\hline
\end{tabular}

xvii 
QCT

SDF

SEF

TEGDMA

THA

TNS

UCS

UD

UFS

UTS
Quantitative computed tomography

Stiffness deviation factor

Stiffness efficiency factor

Triethylene glycol dimethacrylate

Total hip replacement

Twisting moment neutral

Ultimate compressive strength

Unidirectional

Ultimate flexural strength

Ultimate tensile strength 


\section{INTRODUCTION}

\subsection{Background motivation}

The femur (thigh bone) is the longest and strongest bone in human body. Femoral fractures are considered one of the most common bone injuries in both male and female populations [1]. These fractures are observed in all age groups. However, there tends to be an age- and gender-related bimodal distribution of fractures with injuries occurring most frequently in young males after highenergy trauma and in elderly females after falls from standing [2]. Certain medical conditions such as osteoporosis or cancer can weaken the bone and make it more susceptible to breaking. A fracture in femur takes about 3 to 6 months to completely heal, although the healing can start as early as 6 weeks [3]. Such a long healing period can result in misalignment of the fracture ends, leading to malunion or non-union. Therefore, it is crucial that these fractures are fixed using internal fracture fixation devices. There are different types of fixation devices for femoral fractures. The choice of a proper fixation device depends on the type, angle, and location of the fracture. Fractures that occur in the femoral shaft can be fixed with bone fracture plates and intramedullary (IM) nails. However, with the favourable results of IM nails, the use of fracture plates is limited to those cases where IM nailing is technically impractical. For instance, for patients with extremely narrow medullary canal or those with an existing implant, bone fracture plates remain an excellent option. Despite recent advancements in IM nail design, there are still complications associated with these implants including mal-union, non-union, and failure of the nail or screws [4]. The rigid structure of conventional IM nails, usually made of Ti-alloy, can properly stabilise the fracture site. However, an undesirable consequence of such rigid structure is peri-implant osteopenia as their high axial rigidity causes the nail to bear the majority of the load once implanted, reducing the physiologic loading on the bone [5]. The reduction of the mechanical stress on the femur, which is referred to as stress shielding, results in bone resorption over time [6]. In a fractured femur fixed with an IM nail, the stress shielding effect of the implant can cause the density in the bone to be lower than it was pre-operatively. Consequently, the risk of re-fracture in weakened bone increases

once the fixation implant is removed [7]. Although primary bone healing can occur with rigid fixation, the secondary bone healing that is the major type of bone healing requires axial movement of fracture fragments [8]. Reducing the rigidity of the fixation device could favourably increase the load levels at the fracture site, but it may also compromise the stability of the fracture by 
increasing the unfavourable (e.g. shear and torsional) interfragmentary movements, which are detrimental to fracture healing [9]. Therefore, controversies remain regarding the optimal rigidity of the fixation device [10]. On the other hand, reducing the rigidity in a metallic alloy, which is achievable by reducing the cross-sectional area, can significantly increase the stress levels in the structure of the fixation device, making it vulnerable to fatigue failure [2].

Fibre-reinforced composites have been recently used in biomedical implants because of their high strength, low rigidity, and corrosion and fatigue resistance [11]. Most importantly, they have the possibility to be tailored and adapted based on specific requirements through changing the stacking sequence or volume fraction of the fibres [12].

This thesis aims at developing a flexible yet strong composite IM nail made of carbon-fibre/epoxy. It was hypothesized that the proposed composite nail would result in a more desirable condition

for healing, as it would provide sufficient stability and reproduce the strain distribution of an intact femur in the healed stage through increasing the load transferred to the bone. This would subsequently encourage bone regrowth and prevent bone degradation.

\subsection{Research objectives}

The main objective of this thesis is to solve the limitations of current IM nails by using fibrereinforced composite materials (Carbon-fibre/epoxy) in order to provide preferred mechanical environment for fracture healing. The IM nail is supposed to be rigid enough to produce necessary immobilization at the fracture site during ambulation but should not be so rigid that the fracture is shielded from the stresses required to stimulate healing. This main objective will be achieved through the following sub-objectives:

a) Developing closed-form expressions for structural rigidities of composite circular tubes based on the material being used, the stacking sequence of the laminate, the diameter, and the wall thickness of the tube.

b) Investigating the structural couplings (e.g. extension-twist coupling in a composite tube) to be avoided in the design process. 
c) Using the derived expressions for structural optimization of a composite tube by altering the composite stacking sequence and thickness, aiming at minimizing axial rigidity, while maximizing bending and torsional rigidities.

d) Examining the preferred candidate configurations (obtained from the structural optimization process) in an experimentally validated finite element model to evaluate their mechanical performance in transverse and oblique femoral shaft fractures under extreme physiologic loading.

e) Manufacturing the optimized configuration of the composite IM nail.

f) Performing mechanical characterization of the manufactured IM nails (i.e. performing compression, bending, torsion, hardness, moisture absorption, and fatigue tests).

\subsection{Thesis outline}

The current thesis contains eight main chapters. It is comprised of a series of journal papers that were previously published or are currently under review in peer-reviewed journals.

Chapter 1 provides the motivation behind this research and states the hypothesis in the current work. It also contains the goals this thesis sets out to accomplish as well as detailed objectives.

Chapter 2 includes a comprehensive literature review on the concepts and theories related to this thesis. It starts with an introduction to the anatomy of the human femur, the types of femoral fractures, biomechanics of intact and healing bone, and fracture fixation devices that are commonly used to treat femoral shaft fractures. In particular, it includes the current complications associated with rigid metallic fixations as well as a summary of the studies that proposed less-rigid materials for bone fracture fixation purposes. Further, the shortcomings of the previous biomechanical and clinical studies on using composite materials as fixation devices are discussed. A part of the literature review in this chapter was taken from the introduction section of the following published paper that includes a preliminary study on biomechanics of composite IM nails for fixation of femoral shaft fractures.

Samiezadeh S., Tavakkoli Avval P., Fawaz Z., Bougherara H., 2014. Biomechanical assessment of composite versus metallic intramedullary nailing system in femoral shaft fractures: A finite element study. Clinical Biomechanics (Bristol, Avon) 29 (7), 803-810. 
Chapter 3 includes the optimization of a fibre-reinforced composite bone fracture plate made of carbon-fibre/flax/epoxy based on fracture healing requirements. In the first part of the chapter, closed-form expressions for axial, bending, and torsional rigidities derived from the classical laminate theory, and the composite plate was optimized by varying the laminate stacking sequence and the contribution of each material, in order to minimize the axial rigidity and maximize the torsional rigidity for a given range of bending rigidity. In the second part of the chapter, the best configurations were evaluated using a comprehensive finite element model to obtain their mechanical performance in terms of its fracture stability, load sharing, and strength in transverse and oblique fractures. The winning configuration was then selected considering the various mechanical factors that play a role in the fracture healing process. This part of the thesis was performed prior to the optimization of a composite IM nail in order to test the hypothesis that a composite fixation device could be optimized based on the selective stress shielding approach to increase loading at the fracture site while maintaining the fracture stability. The contents of this chapter were published as a journal paper in the Journal of the Mechanical Behavior of Biomedical Materials as follows:

Samiezadeh S., Tavakkoli Avval P., Fawaz Z., Bougherara H., 2015. On optimization of a composite bone plate using the selective stress shielding approach. Journal of the Mechanical Behavior of Biomedical Materials 42, 138-153.

Chapter 4 combines the classical laminate theory and the beam theory to obtain closed-form expressions for axial, bending, and torsional rigidities of a composite tube. The obtained expressions will then be verified using a three-dimensional (3D) finite element model. These expressions will be used in chapter 6 for optimization of a composite IM nail. This chapter was an Appendix to the following journal paper that was recently published in the Journal of Biomechanical Engineering:

Samiezadeh S., Tavakkoli Avval P., Fawaz Z., Bougherara H., 2015. An Effective Approach for Optimization of a Composite Intramedullary Nail for Treating Femoral Shaft Fractures. Journal of Biomechanical Engineering 137 (12), 121001.

Chapter 5 investigates the structural couplings in a composite tube that, if exist, can cause undesirable deformations and interfere with the normal functionality of the component. In particular, this chapter introduces a factor that detects extension-twist coupling in a composite 
tube. The accuracy of the proposed factor in detecting the extension-twist coupling in composite tubes with similar dimensions to IM nails is then examined using a 3D finite element model that takes into account the out-of-plane deformations and interactions among laminate plies. This chapter was taken from the following journal paper that is currently under review in the Journal of Composite Materials:

Samiezadeh S., Fawaz Z., Bougherara H., 2016. Analysis of extension-twist coupling of thickwalled composite circular tubes. Journal of Composite Materials. Under review.

In chapter 6, the structural optimization of the composite IM nail is performed using the equations derived in chapter 4 , the coupling factor introduced in chapter 5 , and a comprehensive finite element model. The optimization process starts by altering the composite stacking sequence and thickness to minimize axial rigidity, while maximizing torsional rigidity for a given range of bending rigidities using the closed-form expressions. The selected candidates are then examined in an experimentally validated finite element model to evaluate their mechanical performance in transverse and oblique femoral shaft fractures. The finite element model consists of the femur, composite IM nail, and locking screws. The best configuration is chosen based on a number of parameters that are involved in fracture healing. The content of this chapter was taken from the following journal paper:

Samiezadeh S., Tavakkoli Avval P., Fawaz Z., Bougherara H., 2015. An Effective Approach for Optimization of a Composite Intramedullary Nail for Treating Femoral Shaft Fractures. Journal of Biomechanical Engineering 137 (12), 121001.

Chapter 7 discusses the mechanical properties of the proposed composite IM nail which was optimized in chapter 6 . This chapter contains the results of a set of quasi-static mechanical testing on the manufactured composite specimens. To investigate the response of the composite nail to cyclic loading during normal daily activities, the fatigue performance of the developed composite IM nail is also investigated in this chapter using the infrared thermography approach. The majority of the materials in this chapter were taken from the following journal paper:

Samiezadeh S., Fawaz Z., Bougherara H., 2015. Biomechanical properties of a structurally optimized carbon-fibrelepoxy intramedullary nail for femoral shaft fracture fixation. Journal of the Mechanical Behavior of Biomedical Materials 56, 87-97. 
Chapter 8 concludes the important findings of the current thesis and outlines the major contributions of the work. It also discusses how the current findings support the hypothesis of the research, and contains the limitations and suggestions for future work.

Chapter 9 is the Appendix of the thesis and contains the computer codes developed in chapters 3 and 6 to perform the optimization procedure. 


\section{LITERATURE REVIEW}

\subsection{Human femur}

The human femur, also referred to as the thigh bone, is the largest and strongest bone in the human body. It is approximately one-fourth of the height of the individual [13]. At the upper (proximal) end it articulates with the pelvis forming the hip joint, and at the lower (distal) end it articulates with the tibia and patella. Figure 2-1 depicts the anatomy of the human femur. The femur primarily consists of the head, neck, greater and lesser trochanter, shaft, and the epicondyle [14]. The femoral shaft, which is so called as diaphysis, is made up of the cortical bone, and hosts the medullary canal which is associated with an interior empty space.
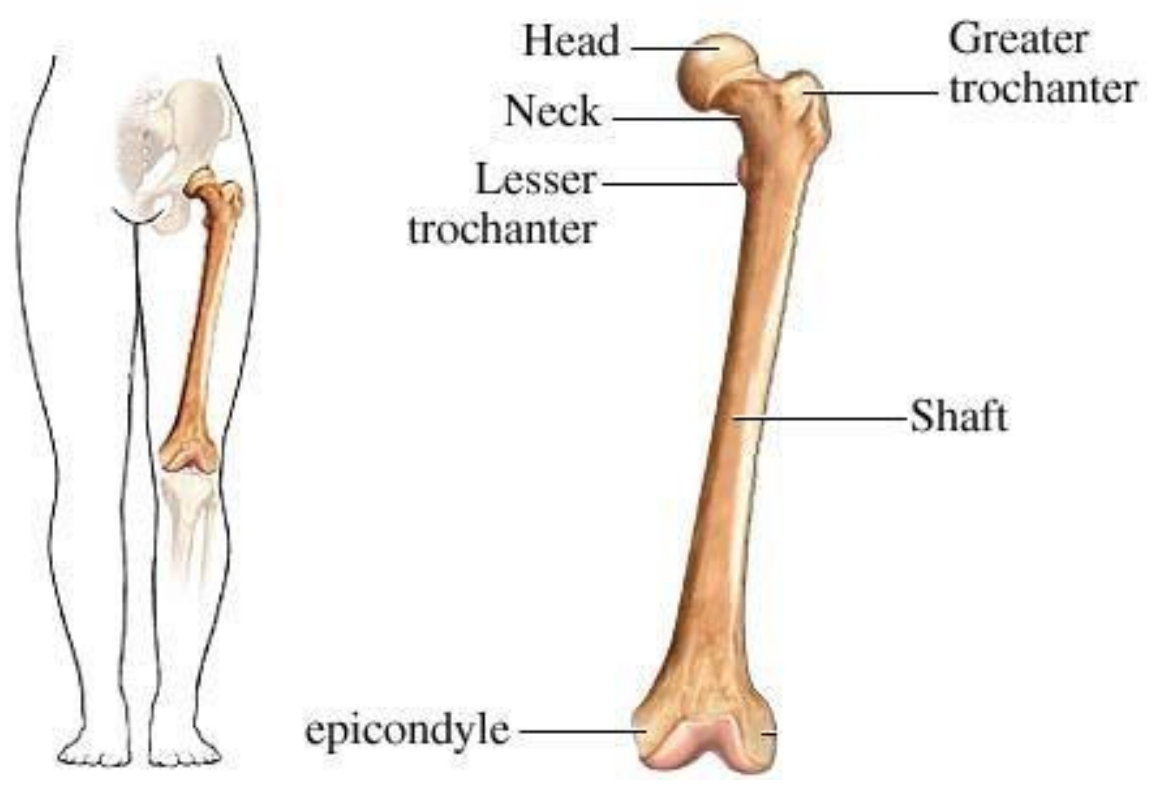

Figure 2-1. Anatomy of the human femur [14]

The femur is subjected to loads that are typically twice the weight of an adult body [2]. There are two main axes in the femur, i.e., the long axis and the neck axis (Figure 2-2). The angle between these axes is referred to as the inclination angle and is approximately $125^{\circ}$ in a healthy femur. 


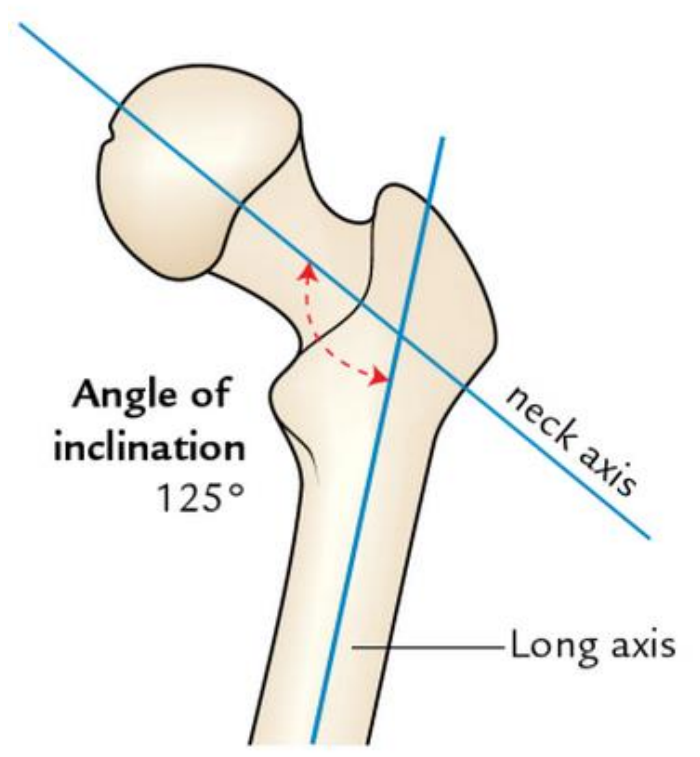

Figure 2-2. The main axes in human femur

The femur, similar to other long bones, contains two distinct morphological types of bone, i.e., cortical (compact) bone, and cancellous or trabecular bone. The former type forms a dense cylinder as the shaft of the bone surrounding the marrow cavity. The latter type is located at the ends of long bones, and has an open and spongy structure. It has a much lower elastic modulus than the cortical bone. Due to the higher density of the cortical bone structure, it counts for approximately $80 \%$ of the mass of bone in the human body [15]. Figure 2-3 illustrates the structure of the femoral bone. 


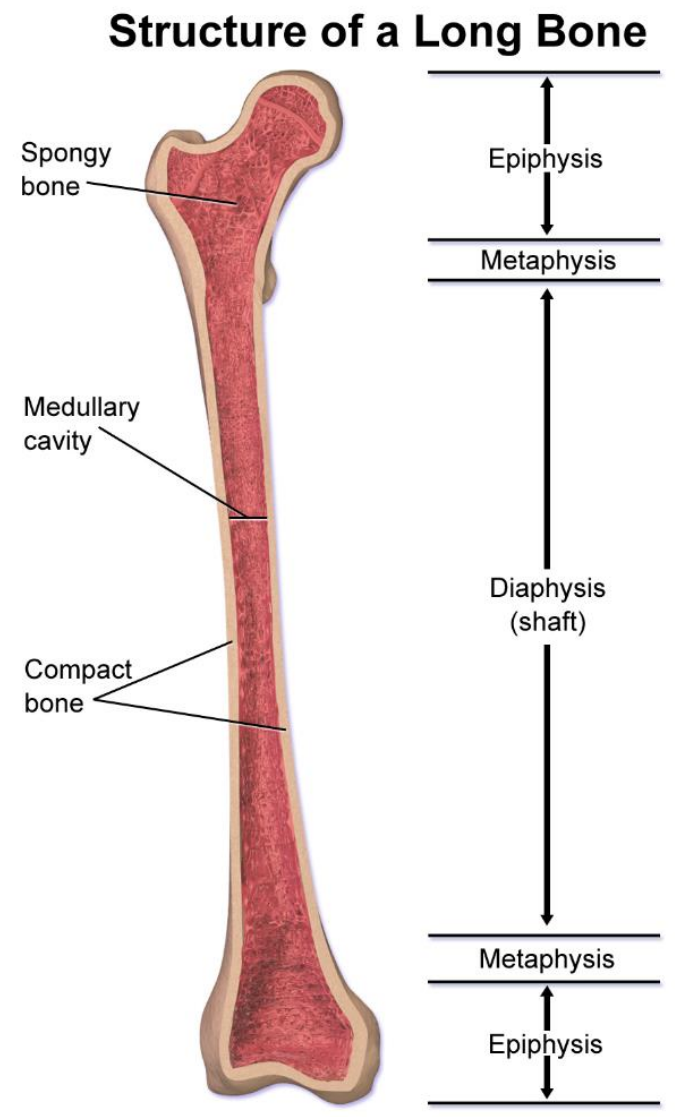

Figure 2-3. Structure of the femoral bone [16].

\subsection{Hip fractures}

Hip fracture is a main concern of the elderly population, most of whom suffer from osteoporosis in addition to various medical and mental diseases that result in them being a high-risk population. It is currently estimated that 250,000 hip fractures occur annually in the United States alone and that this number may double within 40 years [17]. Hip fractures comprise $20 \%$ of the operative workload of an orthopaedic trauma unit [18]. A recent survey [19] on the epidemiology of bone fractures in adults reviewing 5953 cases in a single trauma unit showed that femoral fractures have an overall incidence of about $13 \%$ of all body fractures. These fractures are considered one of the most common bone injuries in men and women [1]. Numerous associated injuries occur in conjunction with fractures of the femoral shaft and are more commonly observed in young patients after high-energy traumatic injuries.

Femoral fractures are observed across all age groups and are attributable to a variety of mechanisms [19-21]. The mechanisms in young patients tend to be motor vehicle crashes, 
motorcycle crashes, pedestrians struck by vehicles, or falls from height. The relative distribution of these fractures depends on multiple factors including the geographic location (urban versus rural) and country of study. In a review of 515 patients with 551 femoral shaft fractures in a typical urban U.S. city, the average age was 27.2 years and 70\% were males [22]. The mechanisms of injury were motor vehicle crashes in $78 \%$, motorcycle crashes in $9 \%$, pedestrians struck in $4 \%$, falls from height in 3\%, gunshot wounds in $2 \%$, and other miscellaneous mechanisms in $3 \%$.

\subsubsection{Femoral fracture classifications}

Femoral fractures can occur at different parts of the femoral bone. They are classified as head fracture, neck fractures, intertrochanteric fractures, subtrochanteric fractures, shaft fractures, and distal fractures.

\section{Femoral shaft fractures}

A femoral shaft fracture is a fracture of the femoral diaphysis occurring between $5 \mathrm{~cm}$ distal to the lesser trochanter and $5 \mathrm{~cm}$ proximal to the adductor tubercle [23]. These fractures have been classified by the anatomic location, fracture morphology, degree of comminution thereof. One of the world-wide accepted classification is done by Winquist-Hansen [24, 25] as depicted in Figure 2-4, and Table 2-1.

Grade 0 fractures have no associated comminution. Grade I fractures have a small chip or fragment of comminution. Grade II fractures have a small butterfly fragment, but at least $50 \%$ of the cortex remains intact. Grade III fractures have a larger butterfly fragment with minimal cortical abutment predicted. Grade IV fractures have no predicted cortical contact between the fracture fragments and are often referred to as segmentally comminuted. Grade 0 and I fractures are stable in length, whereas grade II fractures are at risk for rotational abnormalities; fractures that are grade III and IV require interlocking to prevent shortening and rotational malunion [2, 26]. 

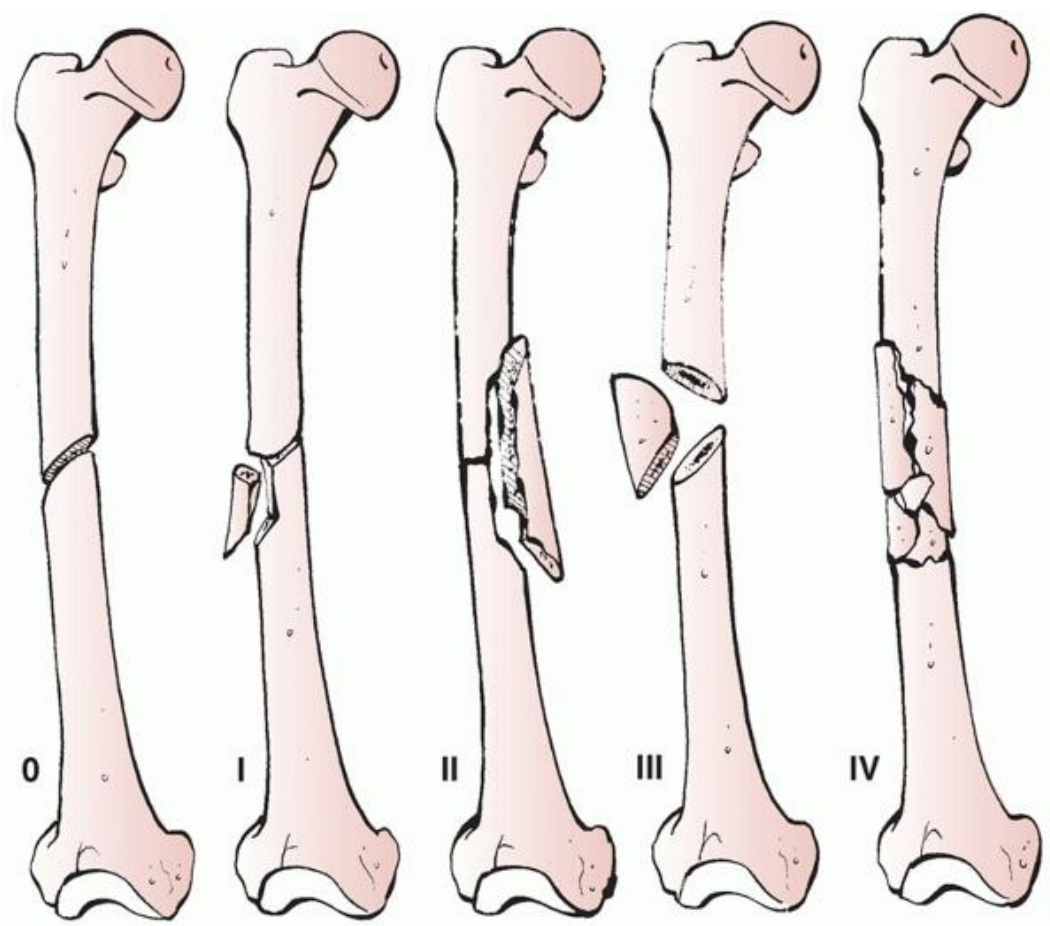

Figure 2-4. The Winquist-Hansen classification for femoral shaft fractures [2]. Refer to Table 2-1 for explanation.

Table 2-1. Winquist and Hansen classification of fracture comminution

\section{Grade Degree of Comminution}

\section{O No comminution.}

I Small butterfly fragment $(<25 \%)$ or minimally comminuted segment with at least $75 \%$ cortical contact remaining between the diaphyseal segments.

II Butterfly fragment or comminuted segment with (approximately $25 \%$ to $50 \%$ ) with at least $50 \%$ cortical contact between the diaphyseal segments.

III Large butterfly fragment or comminuted segment (approximately 50\% to $75 \%$ ) with minimal cortical contact between the diaphyseal segments.

IV Complete cortical comminution such that there is no predicted cortical contact between the diaphyseal segments. Segmentally comminuted. 
Another classification of such fractures is the Orthopedic Trauma Association (OTA) classification which is based largely on the fracture morphology and includes the fracture location as well as the degree and type of comminution (Figure 2-5). Type A fractures are considered simple and include spiral, oblique, and transverse patterns. Type B fractures are wedge fractures and include spiral wedge, bending wedge, and segmental wedge patterns. Type $\mathrm{C}$ fractures are considered complex patterns that have no predicted cortical contact between the major proximal and distal fractures. These fractures are divided on the basis of the same characteristics described for B fractures. Each of these fractures is further divided on the basis of location as subtrochanteric, middle, or distal. Although the precise alphanumeric classification assigned to each femoral fracture is of limited utility, an understanding of the fracture pattern and its location assists with surgical planning and may be useful for documenting and categorizing large numbers of femoral fractures [2]. In a review conducted by Wolinsky et al. [22] on 515 cases, the distribution of fracture comminution according to the scheme of Winquist Hansen was relatively uniform among types 0, I, II, III, and IV. The fracture patterns that could be identified were oblique in $51 \%$, transverse in $29 \%$, comminuted in $14 \%$, and spiral in $6 \%$. 

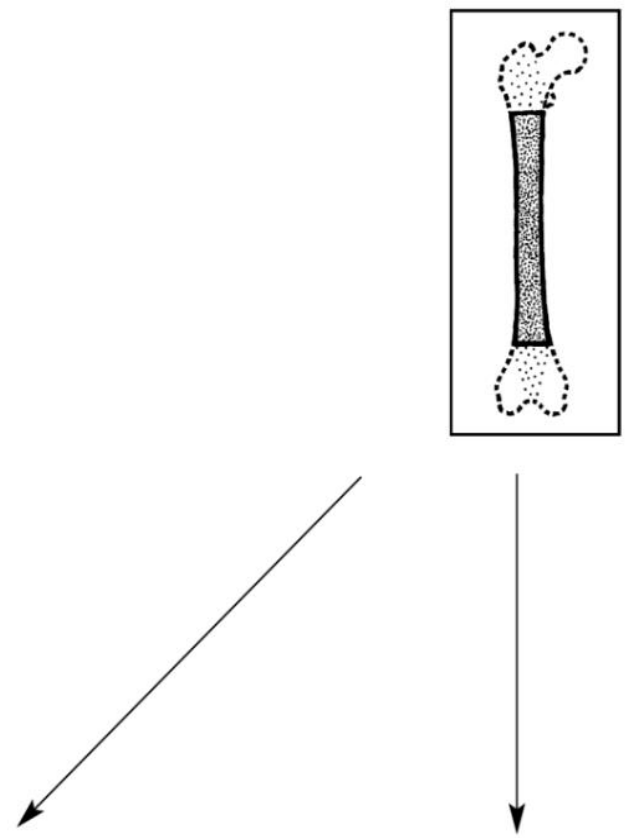

Types:

A. Simple (32-A)

B. Wedge (32-B)
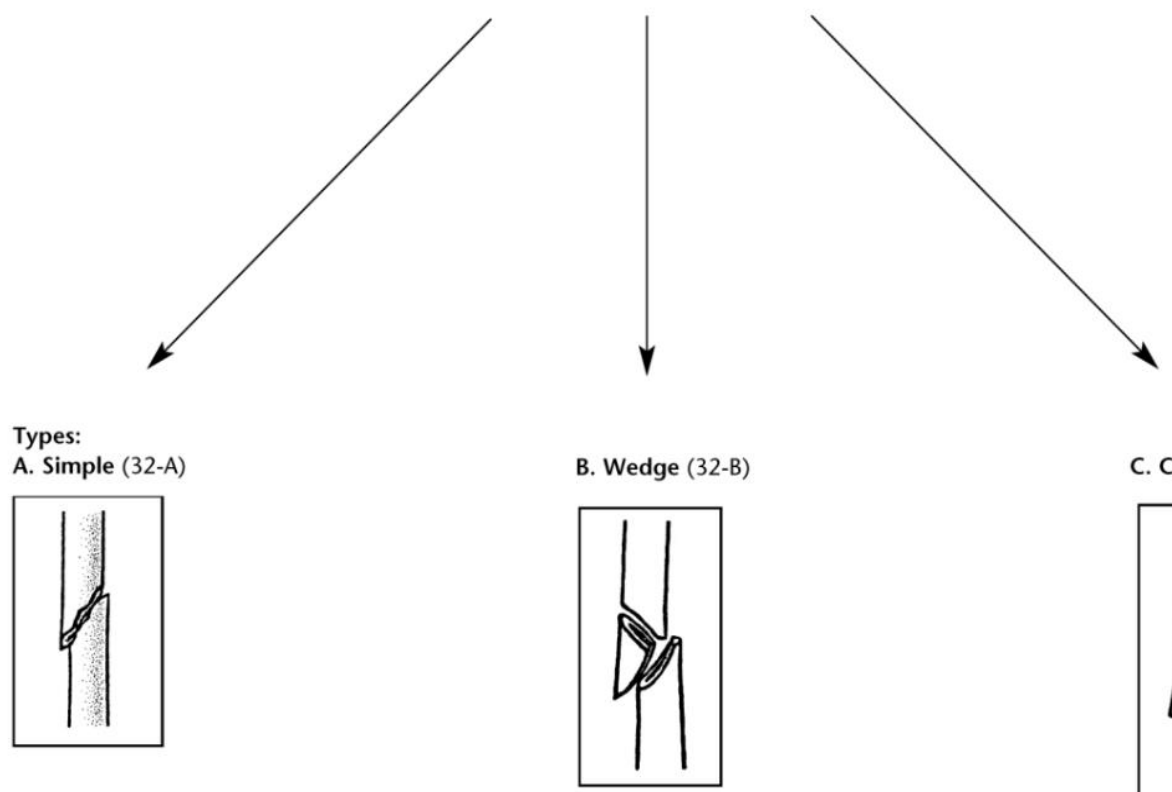

C. Complex (32-C)
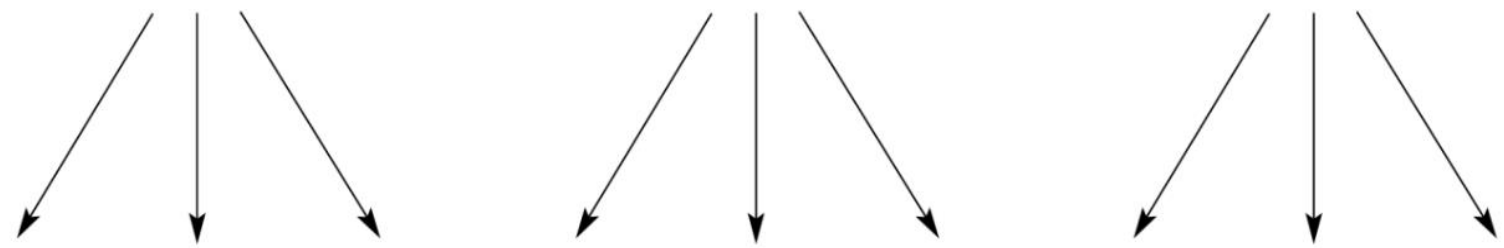

Groups:

Femur, diaphyseal, simple fracture (32-A)

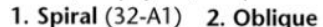

3. Transverse

1. Spiral wedge 2 . Bending wedge (32-B2)

3. Fragmented $\left(\geq 30^{\circ}\right)(32-\mathrm{A} 2) \quad\left(<30^{\circ}\right)(32-\mathrm{A} 3)$

(32-B1)

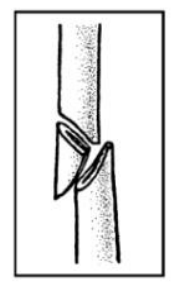

wedge (32-B3)

Femur, diaphyseal, complex (32-C)

1. Spiral (32-C1) 2. Segmental 3. Irregular
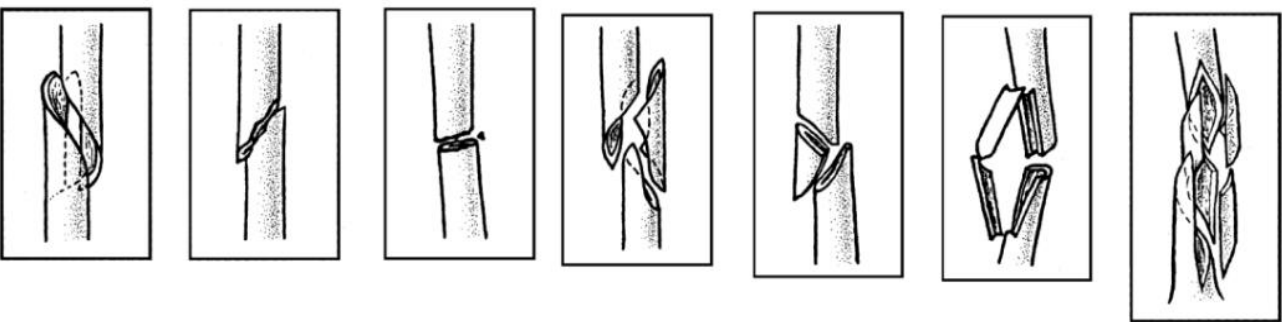
$(32-\mathrm{C} 2)$ (32-C3)

Figure 2-5. OTA classification of femoral shaft fractures [27]. Simple fractures (type A) are distinguished by the degree of obliquity of the fracture line. Wedge fractures (type B) are subclassified according to the anatomy of the wedge fracture. Complex fractures (type C) can be spiral, segmental, or irregular. 


\subsection{Femoral shaft fracture fixation}

Several methods of closed management exist for the treatment of femoral shaft fractures. These include spica casting, traction, cast bracing, or combinations thereof. Currently, closed management as definitive treatment for femoral shaft fractures is largely limited to instances in which devices for internal fixation are unavailable or in patients with significant medical comorbidities that make femoral stabilization impossible [2].

External fixation as a definitive treatment method for femoral shaft fractures has limited indications. Pin tract infections occur commonly and are related to the time the fixator is in place, the amount of soft tissues that the pins must traverse, and the sterility at the time of the initial application [28]. Loss of knee motion occurs commonly. Angular mal-union and femoral shortening occur more frequently than with other methods. There are still concerns about the potential increased infection risk associated with conversion of an external fixator to other definitive treatment methods. Finally, unilateral external fixation has limited ability to adequately stabilize the femoral shaft. This is largely because of the large weight of the leg combined with the distance between the femoral shaft and the bar of the external fixator.

Internal fracture fixation is used to provide bending and torsional rigidity to bone while the fracture heals. Operative stabilization with any technique offers numerous advantages compared with nonoperative methods and includes early patient mobilization and early functional rehabilitation of the injured extremity [2]. The two common operative techniques used in femoral shaft fixation are plating and intramedullary (IM) nailing.

\section{Bone fracture plates}

The use of plate fixation for the routine treatment of femoral shaft fractures has decreased with the increased use of IM nails [2, 26]. With the favourable results of IM nails, the indication for plates has been limited to those cases where IM nailing is impractical or difficult [2]. In patients with extremely narrow medullary canals, fractures around or adjacent to a previous malunion, fractures extending proximally or distally into the pertrochanteric or metaphyseal region, associated vascular injury requiring repair, ipsilateral neck shaft fractures, fractures at or near existing hardware (e.g., peri-prosthetic or peri-implant fractures), and skeletal immaturity, plates remain an excellent option $[2,26]$. The ultimate goal of plate fixation is to stabilize the fracture by driving 
fracture ends together, allowing for both primary and secondary bone healing [2, 29]. In this technique, a plate of appropriate length is attached usually to the lateral side of the femur, bridging the fracture (Figure 2-6). The plate is fixed with a number of screws to the proximal and distal portions of the femur.
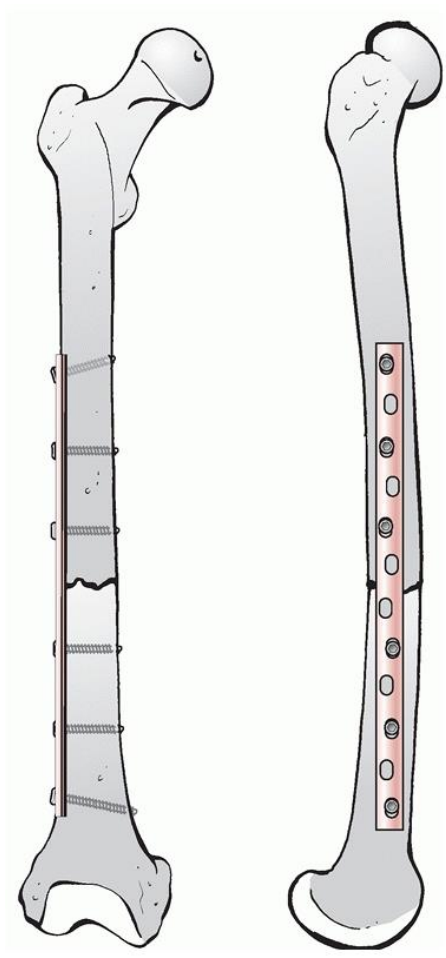

Figure 2-6. Femoral plating for a simple fracture pattern [2]

\section{Intramedullary (IM) nails}

IM nails are the gold standard internal fracture fixation tools used to treat diaphyseal fractures in long bones. They are implanted within the IM canal and fixed by interlocked screws to stabilise the bone fragments during the healing process [26]. Several advantages over other internal fixation devices, such as plates, have made IM nails the golden standard for treating long bone fractures [2]. These advantages are both clinical and mechanical. The mechanical advantages include less stress shielding, lower tensile and shear stresses on the implant and thus a lower rate of implant failure, and the possibility of obtaining a flexible fixation [23]. The clinical advantages include less extensive exposure and dissection, lower infection rate, increased vascularization, and less quadriceps scarring, early mobilization of the patient and thus a marked decrease in the cost of 
hospitalization [26, 30, 31]. Unlike rigid fixation such as plates, fracture treatment with IM nails results in indirect healing characterized by periosteal callus formation and enchondral bone formation [30].

IM nails could be implanted in antegrade or retrograde configurations with static or dynamic interlocking screws fixations (Figure 2-7). Location, angulation, number, and the size of the interlocking screws are variable. The number of distal interlocking screws necessary to maintain the proper length, alignment, and the rotation of the implant-bone construct depends on numerous factors including fracture comminution, fracture location, nail size, patient size, bone quality, and patient activity [2]. In virtually all femoral shaft fractures, however, the definitive management of choice is implanting an anterograde, reamed, locked IM nail [32]. Such placement of IM nails can facilitate transfer of the load and stress through both the fractured femur and the nail. Different metallic alloys such as stainless steel and titanium are being used in manufacturing IM nails.

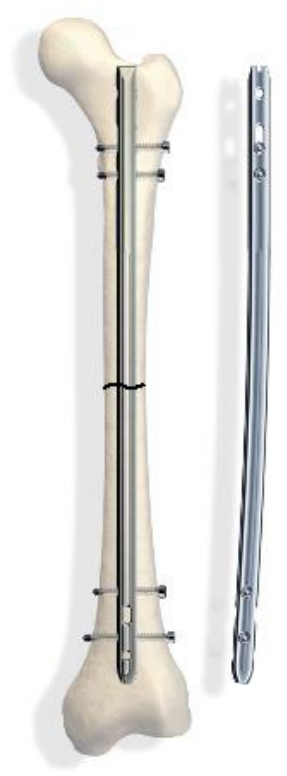

A

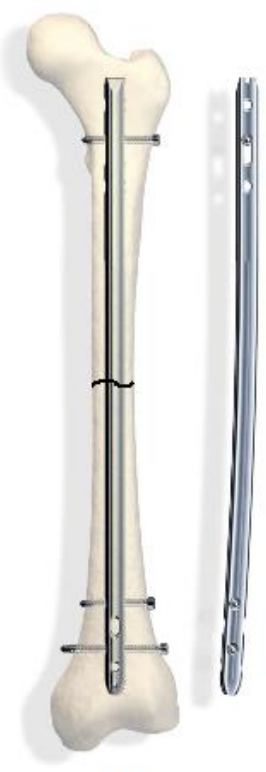

B

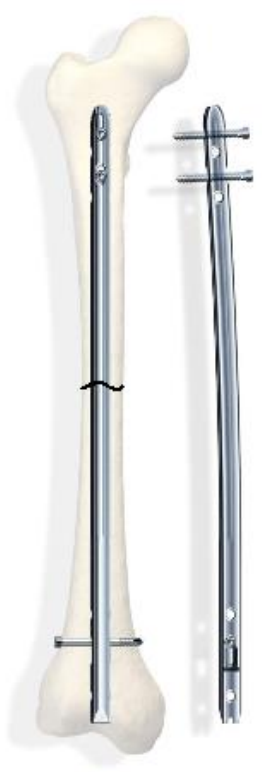

C

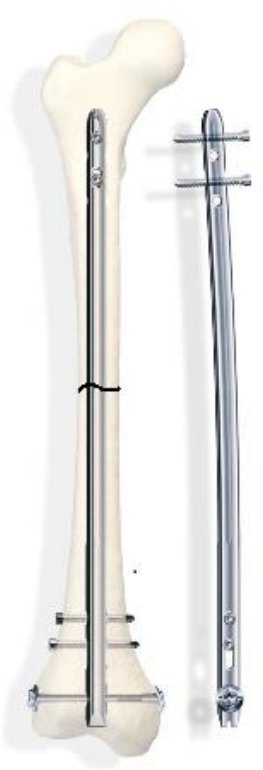

D

Figure 2-7. IM nailing for a simple fracture pattern [33]. A) static antegrade, B) dynamic antegrade, C) static retrograde, D) dynamic retrograde configurations. 


\subsection{Biomechanics of intact and healing bone}

Bone has a hierarchical structure (Figure 2-8). Its lowest level consists of single collagen fibrils with embedded apatite crystals. Changing the collagen to mineral ratio at this level significantly affects the elastic modulus of the bone as it reduces with the loss of mineral [34, 35], as depicted in Figure 2-9. This figure shows Young's modulus as a function of different concentration of HCL. The graph can be used to study the contribution of bone mineral density to the Young's modulus since bone gets demineralized by the exposure of HCL and greater HCL concentration progressively demineralizes bone, ultimately leaving only collagen. This is important from a fracture healing point of view because mineralizing healing callus goes through phases of increasing mineral density and corresponding increased modulus as healing occurs [2].

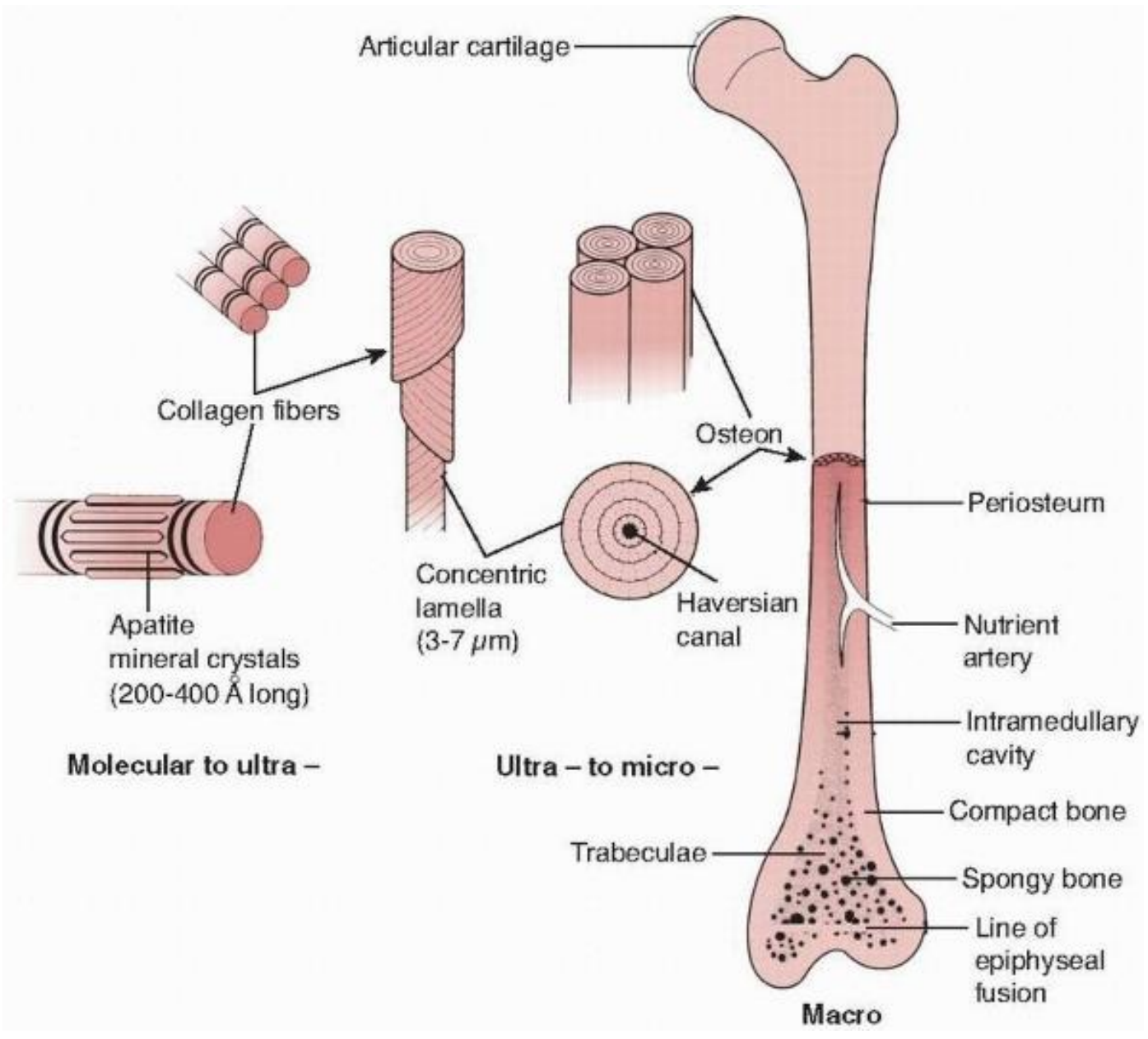

Figure 2-8. The hierarchical structure of bone [2]. 


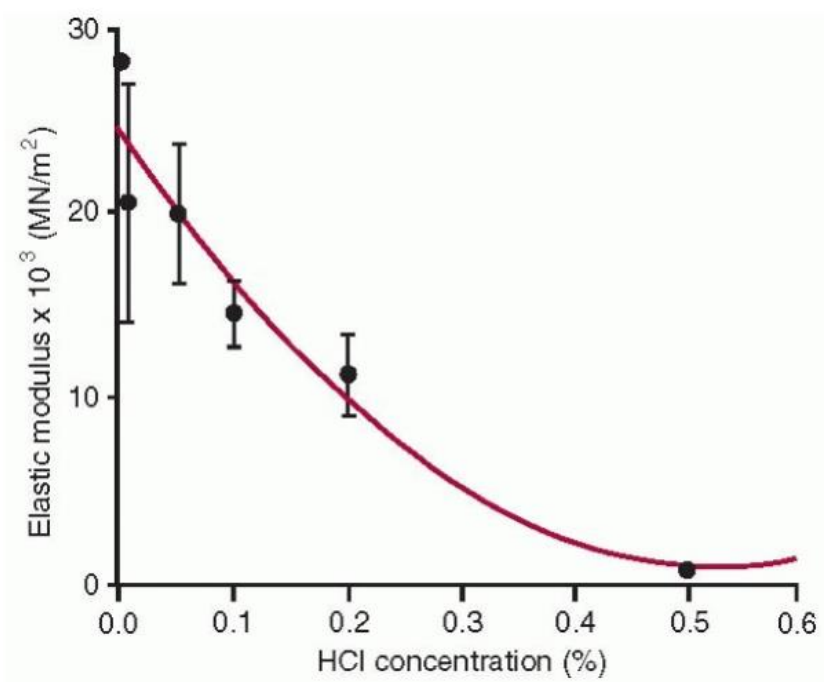

Figure 2-9. Elastic modulus of bone samples tested in tension after exposure to different concentrations of HCL [34].

At the next level of the structure, the orientation of the collagen fibrils is important [36-39], as it affects the ability of the bone to support loads in specific directions (Figure 2-10). During fracture healing process, the callus initially starts as a disorganized random array of fibres, which progressively reorganize to become stiffest along the directions of the major applied loads to which the bone is exposed [2].

At the next level, the density of the haversian systems affects bone strength. Several studies suggested that a power law relationship exists between bone density and strength at this level of structure (Figure 2-11) [35]. Similarly, the Young's modulus changes with bone density by a power of between 2 and 3 [40,41]. As a fracture heals, its strength is affected by changes in its mineral content, callus diameter, and fiber organization. The initial callus forms from the periosteal surface outward, which is beneficial mechanically, because as the outer diameter of the healing area increases, so does its moment of inertia and therefore its initial rigidity [42], as depicted in Figure 2-12. 

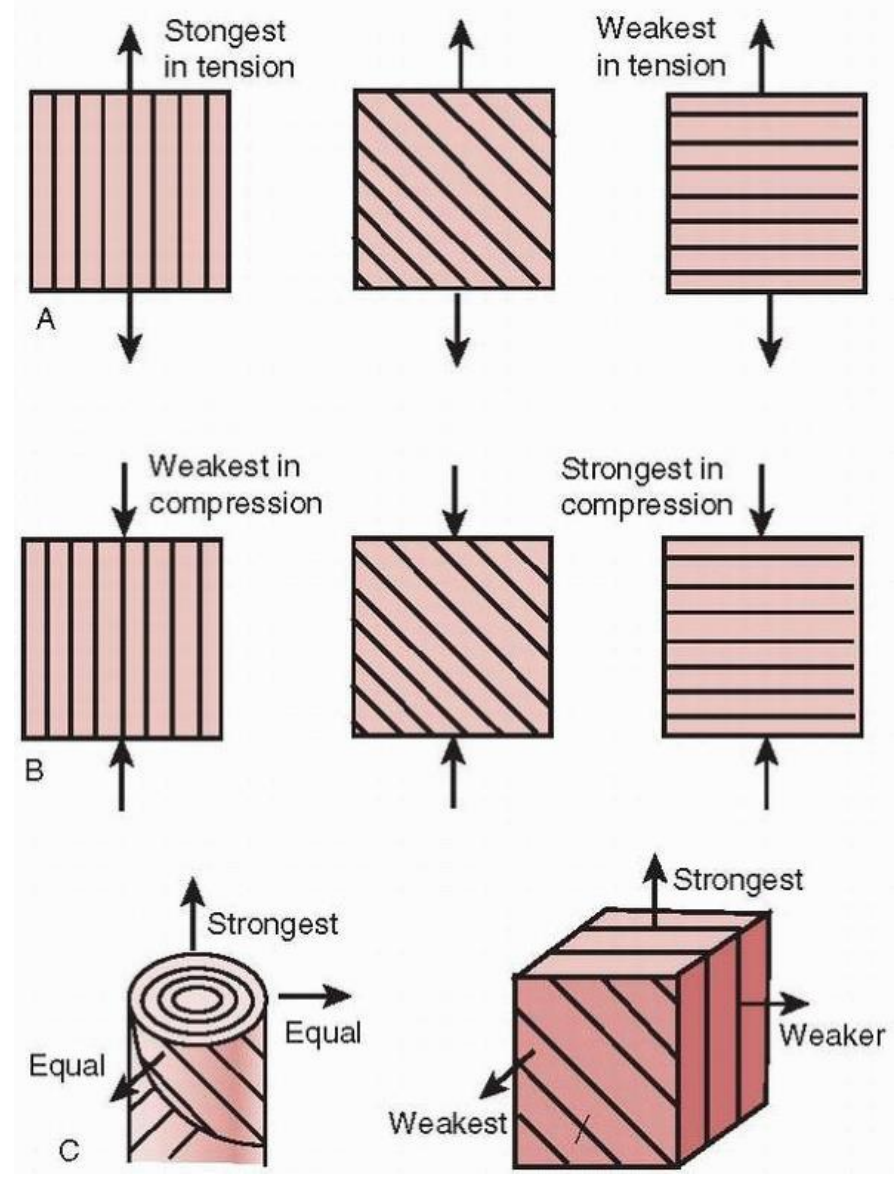

Figure 2-10. Effects of collagen fiber direction on the resistance to loads applied in different directions [39].

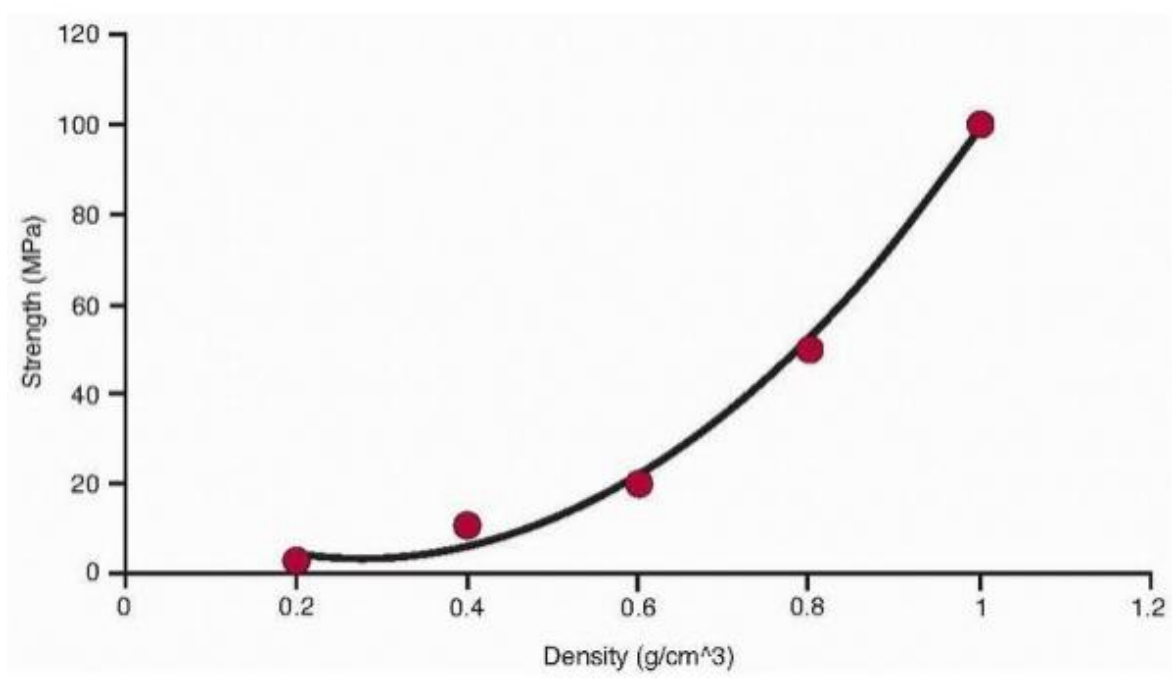

Figure 2-11. The relationship of trabecular bone density to compressive strength and modulus [35]. 

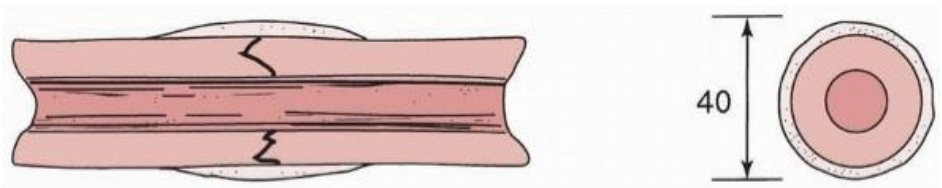

(A) Rel strength $/ \mathrm{mm}^{2} 5 / 3$

Rel rigidity $/ \mathrm{mm}^{2} 2$
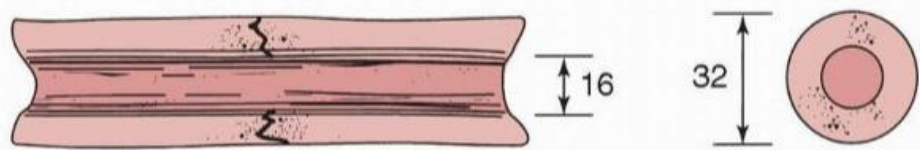

(B) Rel strength/mm² 1

Rel rigidity/mm² 1
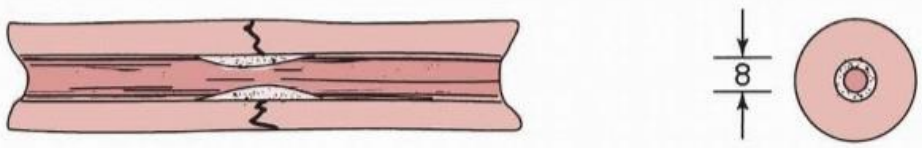

(C) Rel strength/mm $\mathrm{mm}^{2} 1 / 2$

Rel rigidity/mm² $1 / 4$

Figure 2-12. A comparison of the moments of inertia and resulting strengths when fracture callus is located A) on the outer surface, B) on the bone surfaces, or C) in the medullary canal [42].

The cross-sectional area increases progressively up to the peak point and then slowly decreases, as shown in Figure 2-13. There is a similar increase in the mineral content [43]. It is evident from the figure that beyond 4 weeks (in rats, whose bones heal rapidly), the cross-sectional area starts to decrease as the bone remodels to normal shape, while the bone tissue continues to mineralize.

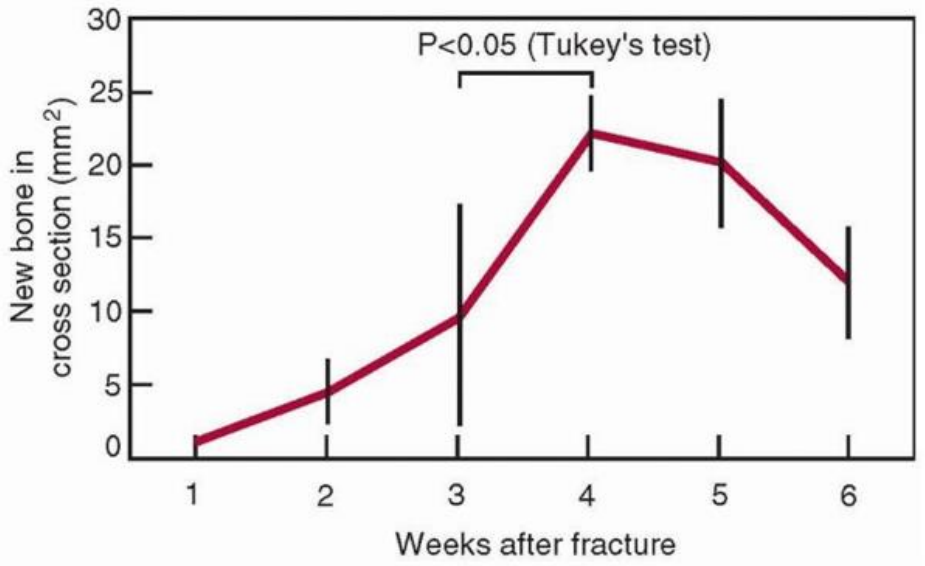

Figure 2-13. Changes in the cross-sectional area of a healing femoral fracture in rats [43]. 
Both rigidity and strength of bone increase with increased duration of healing. Figure 2-14 shows significant increase in both rigidity and peak torque to failure with increased duration of healing in rabbits [44]. In that experiment, the rigidity appeared to gain normal values before the peak torque to failure, suggesting that rigidity and strength are related but not directly.

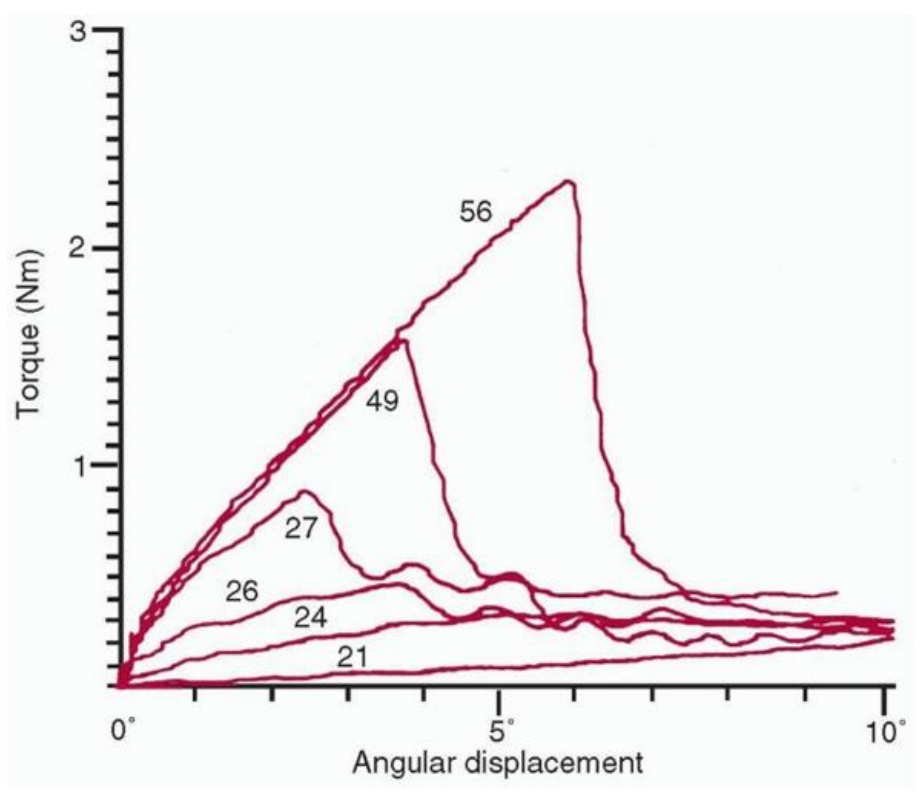

Figure 2-14. Torque versus angular displacement plots taken from experimental long bones at different stages of healing in rabbits. The numbers in the graph show time in days [44].

Fracture healing can be divided into two main categories, i.e., primary (direct) bone healing and secondary (indirect bone healing). Primary bone healing occurs with rigid stabilization (strain is < $2 \%$ ) and does not require compression of the fracture ends. It is direct bone repair without endochondral ossification, which includes cartilaginous callus formation. The main part of this process is Haversian remodeling and re-establishment of blood vessels [8]. Secondary bone healing is characterized by three overlapping stages: the inflammatory response, callus formation, initial bony union and bone remodeling [8]. It occurs in the absence of rigid fixation at the fracture site (strain is $2-10 \%$ ), and it is the more common method of bone healing [45].

The mechanical environment created by the fixation system along with the available blood supply affects the type of tissue formed in a healing fracture. The theory of interfragmentary strain 
attempts to relate the types of tissues formed to the amount of strain experienced by the tissue between the healing bone fragments [42]. This theory is a simple representation and cannot describe the complex stresses that the tissue is exposed to during actual healing. Nonetheless, within the limitations of the theory, when large strains occur in the tissues between healing bone surfaces, granulation tissue is formed. Intermediate level strains produce cartilage and small strains result in primary bone healing, or direct deposition of bone tissue with limited callus formation $[2]$.

Among the limitations of this theory, one should recognize that it does not follow that zero strain will result in maximum bone formation. Load and some resulting strain are necessary within the healing fracture to stimulate bone formation [42]. In a study by White et al. [46] on 24 adult female rabbits, controlled daily displacements in compression were applied to healing long bones using an external fixator, and the bone mineral within the healing fracture was measured with time. It was found that there was an optimal displacement above or below which less mineral was created in the fracture callus, as depicted in Figure 2-15. It is evident that some displacement (in this experiment, $0.5 \mathrm{~mm}$ ) stimulates bone formation, but that greater displacements $(1.0 \mathrm{~mm}$ and 2.0 $\mathrm{mm}$ ) do not enhance bone formation. These results point to an optimal range of displacements for maximum bone formation. Moreover, compression rather than tension is the preferred direction of loading at the fracture site. Fracture fixation constructs of different rigidities within a certain range produce healed fractures with similar mechanical properties, however, they may reach this endpoint by different routes. In a study by Woodard et al. [47] femoral fixation was performed using IM nails by of either $5 \%$ or $50 \%$ of the torsional rigidity of the intact femur. It was found that the femurs fixed with the lower rigidity rods produced an abundance of stabilizing callus, as opposed to the femurs with more rigid fixation. This is mainly due to the fact that there was less necessity for biological fracture stabilization with more rigid fixation. However, in both cases, the mechanical properties of the healed fractures were eventually similar. These studies demonstrate that some strain as a result of loading the fracture site stimulates healing, and that bone will adapt and heal within a relatively wide range of mechanical stability environments. 


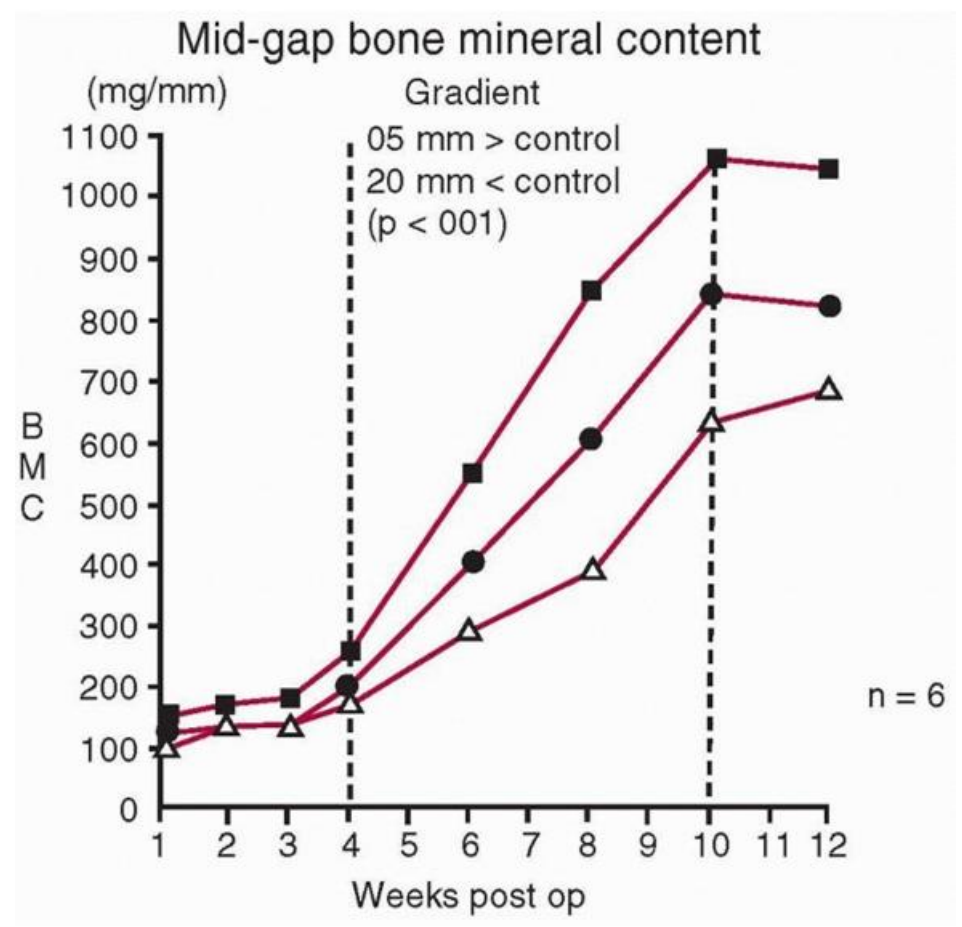

Figure 2-15. The effect on bone mineral content of different cyclic displacements applied daily within a healing fracture (upper curve, $0.5 \mathrm{~mm}$; middle curve, $1.0 \mathrm{~mm}$; lower curve, $2.0 \mathrm{~mm}$ for 500 cycles/day) [46].

In summary, several factors affect the strength of bone and healing fractures. Increasing mineral content increases fracture stiffness. Callus that forms on the periosteal surface is beneficial in increasing the moment of inertia and therefore the rigidity of the fractured region. Healing fractures exhibit several stages, with rigidity returning to normal followed later by peak load to failure. Bone will heal within a range of mechanical environments. To a certain extent, healing bone will compensate for more flexible fixation by forming a greater quantity of fracture callus. However, there is a range of loading of a healing callus, sufficient to stimulate bone formation, which increases as the callus matures [2]. Compressive normal stress at the fracture site has shown to boost fracture healing by stimulating remodelling of the callus, while excessive shear movements may result in delayed union or poor callus quality $[9,48]$. There are controversial results regarding the effect of shear movement on fracture healing. It is assumed that shear movement at fracture sites reduces vascularization and promotes fibrous tissue differentiation [49]. However, natural rapid healing has been observed in oblique fractures treated by functional bracing, where shear movement up to $4 \mathrm{~mm}$ could occur [50]. While some studies reported delayed healing with the 
presence of shear movement at fracture [9], others reported no impairments of healing [51] or even improved healing with shear [52]. The effect of shear movement might be sensitive to the presence of axial motion, timing, and/or gap size [49].

\subsection{The major limitations of conventional IM nails}

The nail's function is to stabilise the fracture fragments, allowing load transfer across the fracture site while maintaining anatomic alignment of the bone [53]. There are, however, complications associated with the use of IM nails as in other internal fracture fixation. These include pain, malalignment, functional impairment, or fracture of the nail or the bone [26, 54].

Conventional metallic nails usually made of titanium (Ti)-alloys or stainless steel can provide appropriate stability at the fracture site. However, an undesirable consequence of such sturdy implants is peri-implant osteopenia as their high axial rigidity causes the nail to bear the majority of the load once implanted, deviating the surrounding bone from the load to which it would be naturally subjected [5]. This removal of normal stress from the bone, which is known as "stress shielding" effect, causes the bone to adapt by reducing its density [6]. This condition makes the bone prone to re-fracture, which is considered a difficult-to-manage complication of plating [55]. Also, bone resorption may cause weakening of the bone-implant interface, thus potentially loosening the implant. The high rigidity of the fixation device can also delay union by reducing compressive loads at the fracture site, thereby inhibiting secondary bone healing [29].

Failure of the construct occurs as breakage of the locking screws, fracture of the nail itself, or damage of the bone around the screw holes [56]. This could be as a result of stress concentrations

and stress shielding following the surgery. Furthermore, the excessive loads carried by the nail as a result of its high rigidity could lead to the failure of the device.

\subsection{Previous attempts to solve the problem of stress shielding}

According to the Mechanostat, the reduction of mechanical stress causes the bone to adapt itself by reducing its mass either by becoming more porous (internal remodeling) or getting thinner (external remodeling) [6]. Much work has being directed into increasing the portion of the load carried by the bone to prevent the bone density from deceasing as a result of bone resorption and to encourage healing. Metal alloys that are commonly used in manufacturing orthopaedic implants, cause the nail to bear the majority of the load once implanted, shielding the bone from the stress it 
would naturally experience [5]. In addition, stress generated within the postoperative femur is often unnatural and has maximum occurring strains significantly larger than that of an intact femur. This is due to the high rigidity of the metallic nail which increases strain generated in adjacent bone as a result of the non-anatomical loading experienced along the femoral shaft. Several studies have shown that less rigid implants reduce the stress shielding and boost the healing process [57, 58]. The superiority of titanium over stainless steel IM nails has been shown in a biomechanical study conducted by Perez et al. [59]. In an animal study conducted by Sha et al. [60], a low-rigidity nail manufactured from a Ti alloy (Ti-24Nb-4Zr-7.9Sn) exhibited better external callus formation, and reduced the effects of stress shielding and bone resorption when compared to a more rigid nail. They also showed that the low-rigidity nail was sufficiently strong to maintain alignment of the fracture in the osteoporotic rat model without delayed union.

In trying to solve the problem of stress shielding, various implants made of so called "isoelastic" materials have been designed [61]. The main challenge here is to design an implant which is flexible enough to diminish stress shielding and, at the same time, rigid enough to provide sufficient level of stabilization and maintain interface micro-motion relatively low [61]. Flexible implant for use in total hip arthroplasty (THA) did not yield mid-term satisfactory results [62, 63], mainly because of the micro-motion at the interface which is believed to have a direct effect of implant failure. Composite materials were initially introduced as they have potential to be manipulated to have combinations of properties that cannot be obtained by conventional materials [64-66].

\subsubsection{Previous studies on composite fracture fixation devices}

There is a growing interest in using fiber-reinforced composites in bioengineering. Several studies have investigated the possible applications of fiber-reinforced composite materials for biomedical implants [64-75]. Factors such as high specific modulus and strength, wear properties, fatigue behaviour, chemical inertness, moisture absorption, their biologic similarities to the human tissue, less stress shielding, and cost play important roles in selecting a robust candidate for use in biomedical applications [61]. One could alter the fiber orientation and properties in fiberreinforced composites to obtain desired axial, torsional and bending rigidity. Manufacturing 3D complex shapes would also be possible by using appropriate molds. 
Carbon fibre reinforced composites have shown promising results in recent animal studies [76]. Of their main advantages is that they are radiolucent (i.e. they have greater transparency to X-ray photons) [77]. This characteristic makes them compatible with modern medical imaging like computed tomography (CT) or magnetic resonance imaging (MRI), which are not possible with current metallic implants. Yet, the complications of using composite materials include reduction of properties over time, degradation of the properties in humified atmosphere, and the risk of fiber breakage [78]. Additionally, there are potential risks to surrounding biological tissue associated with generation of carbon debris from carbon-based composite implants, such as inflammation (i.e., soft tissue thickening), osteolysis (i.e., bone cell resorption), and cytotoxicity (i.e., slowed or halted bone cell proliferation), which might eventually lead to implant loosening and/or failure [79-81].

Several studies reported enhanced healing with the use of more flexible mainly composite bone plates $[55,57,66,79]$. However, excessive flexibility results in poor fixation and subsequent complications, such as malunion or non-union [10, 82-84]. Therefore, controversies remain regarding the optimal fixation rigidity [10,32]. Reducing the rigidity of the fixation plate could favourably increase the load levels at the fracture site and stimulate remodelling of the callus, but it may also compromise the stability of the fracture by increasing unfavourable (e.g. shear and torsional) interfragmentary movements, which are detrimental to fracture healing [9]. Furthermore, stress shielding is not always disadvantageous, and can actually be beneficial in fracture healing, as if the implant adequately counters bending, torsional, and shear stresses while only fractionally resisting compressive stress, it can thereby stimulate remodelling of the callus at the fracture site [85]. This concept is referred to as "selective stress shielding" [48], and is gaining traction in the design of fixation implants as the new generation of materials become available.

In conventional Ti bone plates, axial rigidity could be lowered by reducing the thickness of the structure. However, bending and torsional rigidity will then drop proportionally to the $3^{\text {rd }}$ power of the thickness. Another way of tailoring the rigidity of a metallic bone plate is to make it tubular, as has been done previously in the literature [85]. However, this would increase the stress in the structure, making it prone to fatigue failure [2]. In contrast, a composite material has the potential to be tailored and adapted based on specific requirements through changing the arrangement or volume fraction of the fibres [12]. Studies have been performed to develop polymer-based composite materials as bone implants using natural or synthetic fibres $[81,86]$. They have mainly 
focused on developing hip prosthesis or bone plates. Ali et al. [79] conducted a comprehensive study on developing carbon fibre bone fracture plates. In the first section of their study, they compared the mechanical properties of a carbon fibre composite fracture plate with those of stainless steel and titanium, and in the second section they assessed the cytotoxicity of the proposed plate and reported clinical results from implantation of the nail in 40 human forearms. They found that the proposed carbon fibre plate provides adequate stability, and stress sharing by the bone. The composite plates enhanced callus formation and thus resulted in much earlier union. They did not find a sever reaction from the surrounding tissues, but detected carbon fragment following nail removal. Fujihara et al. [58] proposed a braided carbon/PEEK composite structure for bone plate applications and investigated the effect of plate thickness on plate bending rigidity. Later, Huang et al. [87] compared the bending rigidity of six composite bone plates (using unidirectional (UD) fibre prepregs, braided fabrics, and knitted fabrics), and concluded that a bone plate made of small angle braided fabrics can lead to the best mechanical performance.

Several studies showed increased bone stress with the use of composite bone plates through computer simulations or ex-vivo testing [88-91]. Kim et al. [92] used the finite element method to examine the fracture gap strain distribution in a fractured tibia fixed with five different composite bone plates, and compare it to that in a fractured tibia fixed with a stainless steel plate. Later, Kim et al. [93] investigated the biomechanical performance of the aforementioned composite bone plates in tibial oblique shaft fractures using the finite element method. Both studies evaluated fracture healing rates by considering interfragmentary strain and time-dependent properties for callus, and concluded that a composite bone plate can provide appropriate strain distribution at the fracture site for both transverse and oblique fractures. However, in addition to simplified assumptions about the bone geometry, these investigations did not consider the effect of non-axial loads on fracture. They furthermore did not investigate the failure behaviour of composite bone plates. Recently, Bagheri et al. [81] developed a hybrid composite bone plate made of CF/epoxy prepregs with a flax/epoxy core and evaluated its mechanical performance [91] and fatigue behaviour [94]. However, the effects of various plate rigidity (i.e. axial, bending and torsional rigidity) on fracture stability (i.e. interfragmentary motions), fracture healing, and the strength of the composite structure in physiological loading were overshadowed in previous works that simply aimed to develop a "less rigid" bone plate. Moreover, to date, no optimization has been performed on the composite plate structure to fulfill the requirements of fracture stability and fracture healing. 
There are very few studies on the use of composite materials for IM nails [95, 96]. In one recent study, Moritz et al. [95] developed composite IM nails made of E-glass fibres and bisphenol A dimethacrylate (BisGMA) and triethylene glycol dimethacrylate (TEGDMA) resin. Braided glass fibre sleeves were added to the unidirectional (UD) structure to enhance its properties in bending and torsion. The composite IM nails were then used to fix fractured femurs of 14 rabbits. They harvested the femurs after 12 weeks of follow-up and compared the torsional strength of healed femurs with that of the contralateral intact femur of same rabbit which served as the control group. They found that the healed bone had $83 \%$ of the torsional strength of the contralateral femurs, and did not observe any implant debris or adverse tissue reaction following fixation. The findings of their study suggested the possibility of using fibre-reinforced composite IM nails to fix diaphyseal fractures. However, they only considered two types of structure for their IM nail and no optimization was performed on the nail structure to fulfill the requirements of a proper healing.

To date, however, no study has considered a carbon fiber reinforced composite for use in IM nails. In 2009, a patent was proposed by Metzinger et al. [97] in which the possible geometry of a composite IM nail was presented. However, no details were provided regarding the type and the configuration of the composite material the nail is supposed to be made of. Those few studies [95] that considered the use of composite IM nails for long bone fractures did not perform any optimization on the structure of the IM nail to fulfil the mechanical requirements of fracture healing.

\subsection{Shortcomings of previous biomechanical studies on IM nailing}

Any effort in reducing stress shielding occurred in IM nailing in femoral shaft fractures requires a deep understanding of the biomechanics of fracture fixation and fracture healing in long bones. Several mechanical factors are involved in fracture healing. These include the rigidity of the fixation device, the fracture configuration, the type and the amount of the stresses occurring at both fracture ends, and the relative motion of the fracture fragments $[9,29,53]$. While several researchers have investigated the biomechanics of IM nailing in long bones through simulations and biomechanical testing, few have considered the main factors involved in fracture healing. Cheung et al. [5] conducted a finite element study in which the load sharing patterns of a retrograde IM nailing system at four different stages of gait cycle was investigated. They found that $28 \%$ and $9 \%$ of the axial force is carried by the nail in $45 \%$ and $70 \%$ of the gait cycle respectively. Moreover, 
the use of a stainless steel IM nail results in significantly lower loads in the bone when compared to titanium nail. They also found high level of stress concentrations at the vicinity of screw holes which would suggest the possibility of future fractures. Bougherara et al. [56] performed a biomechanical study on the IM nails implanted in anterograde and retrograde configurations by various locking techniques. They found a moderately good agreement (11.6-33.5\% discrepancy) between the strains obtained from FE simulations and the experimental results. Neither of the studies, however, considered the fracture in the bone and focused on fully healed stage only. Another limitation was that they both ignored the interaction between the IM nail and the inner wall of medullary canal. In the latter study, an axial load was applied on the bone and the forces coming from other muscles were ignored. Montanini et al. [1] investigated stress/strain distribution in a composite femur implanted with an anterograde IM nail by means of FE simulations and experiments. They concluded that the patient underwent IM nailing should avoid full weight bearing due to the high level of stress generated around the proximal screws. Their study had several limitations; only transverse mid-shaft fracture was studied and other fracture types and locations were ignored. Their conclusion about avoiding the full weight bearing following the surgery was based on a relatively large gap in their model $(3.2 \mathrm{~mm})$ which caused the nail to carry a large portion of the load. Their results disagree with several clinical observations [98, 99] that found early weight bearing safe when locked anterograde IM nail is used. Another study that modeled the fracture in IM nailing was conducted by Shih et al. [100], in which static and dynamic fixations in IM nailing were compared using FE analyses. They suggested the used of dynamic technique as it may increase the contact area and pressure at fracture site by looking at a two proximal and distal shaft fractures. The authors omitted the proximal screws in their dynamic fixation configuration which is not clinically the case in dynamic fixation. Moreover, they did not assess the stability of the structure at all and also did not investigate the mid-shaft fractures. Last but not least, they did not verify their numerical results by any means.

A large number of mechanical factors are involved in fracture healing. The most dominant ones are the fracture type and geometry, and the magnitude, direction, and history of the interfragmentary movements [29, 30]. To date, no comprehensive study has investigated the biomechanics of IM nailing employing realistic boundary conditions (i.e. considering muscle forces), and the major mechanical factors involved in the nail performance and fracture healing. 


\subsection{Motivation}

Despite the recent advancements in design and manufacturing of IM nails, several complications remain. The major limitation seems to be their high rigidity, which lowers the physiologic loads carried by the fractured bone, once fixed with an IM nail, as a result of stress shielding induced by the rigid fixation device.

The lack of compressive normal stress at the vicinity of the fracture could result in delayed union, and the increases level of stresses in the nail would increase the risk for nail failure. Even after healing, the lower stresses in the shielded bone increases the chance of bone resorption leading to possible bone re-fracture or implant failure.

Employing a new material which can provide enough stability and, at the same time, minimally affect the internal load patterns in the fractured bone could result in faster union, and a lower rate of implant failure and bone re-fracture. The tailorability, biocompatibility, high strength-to-weight ratio, wear resistance, corrosion resistance, fatigue life, and the radiolucency of fibre-reinforced composite materials have made them one of the best candidates for use in fracture fixation devices. The motivation of the study is to develop a composite IM nail (made of a carbon fibres) to be used in treating femoral shaft fractures. The nail is supposed to provide sufficient stability for healing and reduce the stress shielding induced by the fixation device. The idea is to increase the normal stress at the fracture site to boost healing and to keep the loading patters within the healed bone as close as possible to those in the intact one to reduce the chance of further complications such as bone re-fracture. There are very few studies that considered composite materials for IM nail applications. Moreover, to the author's knowledge, no previous study has considered the optimization of a composite IM nail based on fracture healing requirements. This is also the first study that has used a comprehensive finite element simulation in assessing fracture fixation devices, which takes into account different fracture angles and locations, and mechanical factors involved in the healing process. 


\section{OPTIMIZATION OF A COMPOSITE BONE PLATE USING THE SELECTIVE STRESS SHIELDING APPROACH}

\subsection{Introduction}

Optimization of a fibre-reinforced composite bone plate was performed prior to optimizing a composite IM nail in order to test the hypothesis that a composite fixation device could be optimized based on the selective stress shielding approach to increase loading at the fracture site while maintaining the fracture stability. Both fracture plate and IM nail are used for treating femoral shaft fractures, although the use of fracture plates is limited to those cases where nailing is technically impractical. The structural optimization procedures are similar for a composite bone plate and a composite IM nail. However, closed-form expressions for laminate axial, bending, and torsional rigidities, which are required for optimization purposes, could be derived much more easily for a composite rectangular beam compared to a composite circular tube. There are a number of guidelines that must be followed when designing a fixation device for treating long bone fracture. For instance, similar to an IM nail, the structure of a bone fracture plate must be coupling free, e.g., applying compressive load must not cause twist in the plate structure. Such guidelines can be obtained and verified in the design process of a composite bone fracture plate and then be used in the development of a composite IM nail.

Since both fracture plates and IM nails are used to treat long bone fractures, development of finite element models would be similar. For instance, obtaining muscle forces and muscle attachments, the assumptions in contact modeling, and in particular defining the output parameters in the finite element models that can relate clinical factors to measurable quantities in the model would be similar for both fixation devices. Overall, this chapter acts as a baseline for chapters 4-6, in which development of a composite IM nail will be performed.

To date, no optimization has been performed on the composite plate structure to fulfill the requirements of fracture stability and fracture healing. The purpose of this chapter was therefore to optimize a composite (carbon fibre (CF)/flax/epoxy) structure for use in bone plate applications, based on the selective stress shielding approach and data available from the literature. This combination has been proposed previously as an alternative material of choice for bone plate applications aiming to reduce the adverse effect of stress shielding induced by a rigid implant [81]. 
The optimization process was performed using equations derived from classical laminate theory. The strength of the plate and the effect of plate rigidity on the beneficial factors for healing, in addition to fracture stability, were then investigated using an experimentally validated finite element model. A Vancouver B1 fracture, classified as a stable periprosthetic femoral fracture about the tip of the hip implant following total hip replacement, was considered in the simulations. This type of fracture represents more than $80 \%$ of all periprosthetic femoral fractures, and is commonly fixed with a long bone fracture plate $[2,91]$.

\subsection{Methodology}

\subsubsection{Derivation of rigidity formulae}

The bone fracture plate was treated as a composite beam and analysis was based on the results of the classical laminate theory. The analyses are based on the laminate plate theory and contain some approximations which mainly result from plane-stress conditions. The theory assumes that the strains vary linearly across the laminate thickness and that the out-of-plane shear deformations are negligible [101].

The resultant forces and moments per unit length acting on a laminate cross section are defined as follows [102]:

$$
\begin{aligned}
& {\left[\begin{array}{l}
N_{x} \\
N_{y} \\
N_{x y}
\end{array}\right]=\left[\begin{array}{lll}
A_{11} & A_{12} & A_{16} \\
A_{12} & A_{22} & A_{26} \\
A_{16} & A_{26} & A_{66}
\end{array}\right]\left[\begin{array}{l}
\varepsilon_{x}{ }^{0} \\
\varepsilon_{y}{ }^{0} \\
\gamma_{x y}{ }^{0}
\end{array}\right]+\left[\begin{array}{lll}
B_{11} & B_{12} & B_{16} \\
B_{12} & B_{22} & B_{26} \\
B_{16} & B_{26} & B_{66}
\end{array}\right]\left[\begin{array}{l}
\kappa_{x} \\
\kappa_{y} \\
\kappa_{x y}
\end{array}\right]} \\
& {\left[\begin{array}{l}
M_{x} \\
M_{y} \\
M_{x y}
\end{array}\right]=\left[\begin{array}{lll}
B_{11} & B_{12} & B_{16} \\
B_{12} & B_{22} & B_{26} \\
B_{16} & B_{26} & B_{66}
\end{array}\right]\left[\begin{array}{l}
\varepsilon_{x}^{0} \\
\varepsilon_{y}^{0} \\
\gamma_{x y}{ }^{0}
\end{array}\right]+\left[\begin{array}{lll}
D_{11} & D_{12} & D_{16} \\
D_{12} & D_{22} & D_{26} \\
D_{16} & D_{26} & D_{66}
\end{array}\right]\left[\begin{array}{l}
\kappa_{x} \\
\kappa_{y} \\
\kappa_{x y}
\end{array}\right]}
\end{aligned}
$$

where $A, B$, and $D$ are the extensional rigidity matrix, coupling rigidity matrix, and bending rigidity matrix respectively. For symmetric laminates, forces and moments are decoupled in equations (3.1) ) and (3.2) since $B_{i j}=0$, leading to: 


$$
\begin{aligned}
& {\left[\begin{array}{l}
N_{x} \\
N_{y} \\
N_{x y}
\end{array}\right]=\left[\begin{array}{lll}
A_{11} & A_{12} & A_{16} \\
A_{12} & A_{22} & A_{26} \\
A_{16} & A_{26} & A_{66}
\end{array}\right]\left[\begin{array}{l}
\varepsilon_{x}{ }^{0} \\
\varepsilon_{y}{ }^{0} \\
\gamma_{x y}{ }^{0}
\end{array}\right]} \\
& {\left[\begin{array}{l}
M_{x} \\
M_{y} \\
M_{x y}
\end{array}\right]=\left[\begin{array}{lll}
D_{11} & D_{12} & D_{16} \\
D_{12} & D_{22} & D_{26} \\
D_{16} & D_{26} & D_{66}
\end{array}\right]\left[\begin{array}{l}
\kappa_{x} \\
\kappa_{y} \\
\kappa_{x y}
\end{array}\right]}
\end{aligned}
$$

To obtain the axial rigidity (EA), an axial force of $\bar{N}$ was applied at the centroid of the laminate (Figure 3-1a). To apply the laminate plate theory, $N_{x}$ was defined as the force per unit length (i.e. the width of the beam) by using the following expression:

$$
N_{x}=\frac{\bar{N}}{b}
$$

in which $b$ is the width of the beam. Assuming that $N_{x}$ is the only force component applied on the laminate (i.e. $N_{y}=N_{x y}=0$ ), (3.3) can be rearranged as:

$$
\left[\begin{array}{l}
\varepsilon_{x}{ }^{0} \\
\varepsilon_{y}{ }^{0} \\
\gamma_{x y}{ }^{0}
\end{array}\right]=[A]^{-1}\left[\begin{array}{l}
N_{x} \\
0 \\
0
\end{array}\right]
$$

which yields:

$$
\varepsilon_{x}^{0}=A_{11}^{*} N_{x}
$$

where $A_{i j}{ }^{*}$ are the elements of the $[A]^{-1}$ matrix. The mid-plane strain in the x-direction is:

$$
\varepsilon_{x}^{0}=\frac{\partial u_{0}}{\partial x}
$$

where $u_{0}$ is the displacement of the mid-plane in the $x$-direction, and is generally a function of $x$ and $y$. However, in the case of a narrow beam, i.e., when the length to width ratio is sufficiently high (>10), we can assume that $u_{0}=u_{0}(x)$. Equation (3.7) can then be written as follows: 


$$
\varepsilon_{x}^{0}=\frac{d u_{0}}{d x}=A_{11}^{*} N_{x}
$$

Comparing (3.8) with the axial strain of an isotropic beam (i.e. $\frac{d u}{d x}=\frac{N}{E A}$ ) yields the axial rigidity of a rectangular symmetric composite beam with orthotropic laminae, as follows:

$$
E A=\frac{b}{A_{11}{ }^{*}}
$$

To obtain the bending rigidity $\left(E I_{y y}\right)$, a pure bending moment was applied in the $x$-z plane $\left(\bar{M}_{y}\right)$ (Figure 3-1a) and the moment per unit length was defined as $M_{x}=\frac{\bar{M}_{y}}{b}$. This produces the $x$ component of the stress $\left(\sigma_{x}\right)$ :

$$
\left[\begin{array}{l}
\kappa_{x} \\
\kappa_{y} \\
\kappa_{x y}
\end{array}\right]=[D]^{-1}\left[\begin{array}{l}
M_{x} \\
0 \\
0
\end{array}\right]
$$

which yields,

$$
\kappa_{x}=D_{11}^{*} M_{x}
$$

where $D_{i j}{ }^{*}$ are the elements of $[D]^{-1}$ matrix. The mid-plane curvature in the $x-z$ plane and the outof-plane curvature are defined by Agarwal et al. [102] as:

$$
\begin{gathered}
\kappa_{x}=-\frac{\partial^{2} w_{0}}{\partial x^{2}} \\
\kappa_{x y}=-2 \frac{\partial^{2} w_{0}}{\partial x \partial y}
\end{gathered}
$$

Where $w_{0}$ is the displacement of the beam in the $z$-direction and is a function of $x$ and $y$. However, in the case of a narrow beam, we can assume that $w_{0}=w_{0}(x)$ [101]. Equation (3.12) can then be written as: 


$$
\kappa_{x}=-\frac{d^{2} w_{0}}{d x^{2}}=D_{11}^{*} M_{x}
$$

Comparing (3.14) with the curvature of an isotropic rectangular beam (i.e. $\frac{d^{2} w}{d x^{2}}=-\frac{M}{E I}$ ) yields the bending rigidity of a rectangular symmetric composite beam with orthotropic laminae, as follows:

$$
E I_{y y}=\frac{b}{D_{11}{ }^{*}}
$$
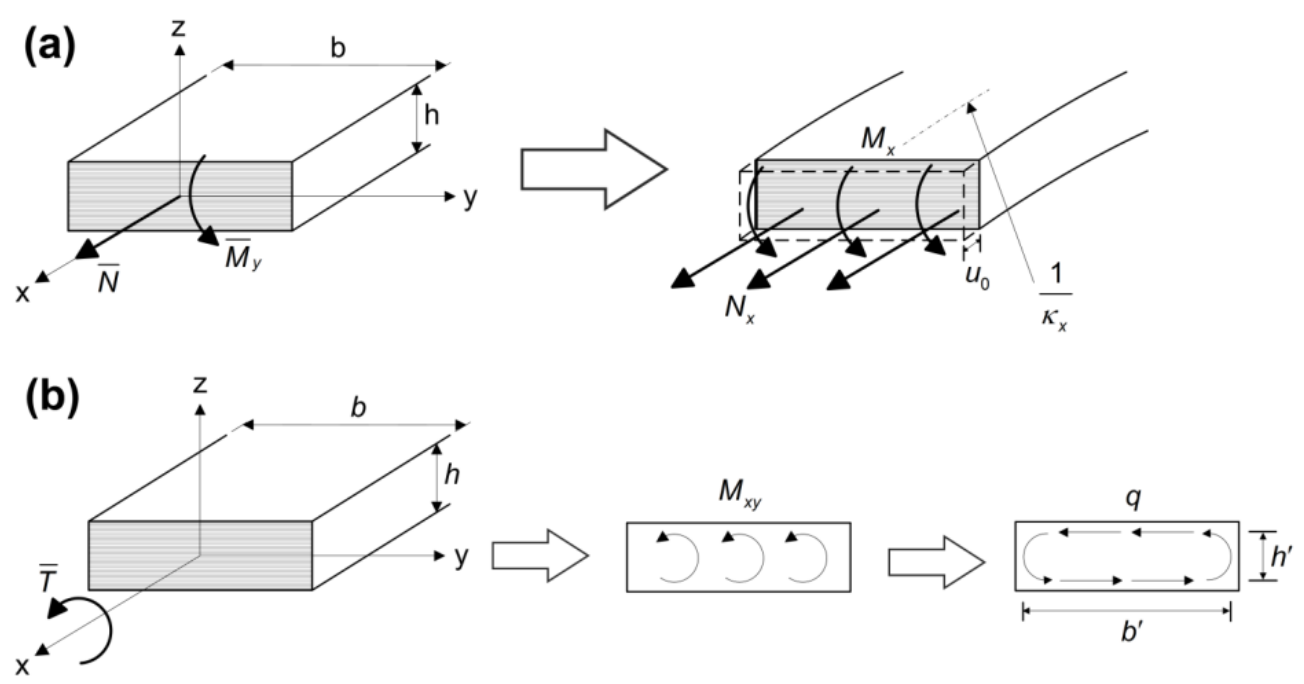

Figure 3-1. Rectangular laminated beam subjected to (a) axial load and bending moment, (b) twisting moment $\bar{T}$ and the resultant shear flow

To obtain the torsional rigidity of the rectangular cross section of the plate, we assume that any applied twisting moment $(T)$ on the beam results in a twist moment $\left(M_{x y}\right)$ and subsequent shear flow $(q)$ (Figure 3-1b), which has units of force per length. The applied twisting moment could be written in terms of the shear flow as follows [101]:

$$
\bar{T}=2 q h^{\prime} b^{\prime}
$$


where $h^{\prime}$ and $b^{\prime}$ are the length and the path of the resultant shear flow (Figure 3-1b). The latter could be considered approximately equal to the width of the plate (i.e. $\left.b^{\prime} \approx b\right)$. The twist moment $\left(M_{x y}\right)$ per unit length in the rectangular cross section is:

$$
M_{x y}=-q h^{\prime}
$$

Combining equations (3.16) and (3.17) yields:

$$
\bar{T}=-2 b M_{x y}
$$

Recalling (3.4) and considering the fact that no axial force or bending moment are applied on the beam, the out-of-plane curvature of the plate $\kappa_{x y}$ would be:

$$
\begin{aligned}
& {\left[\begin{array}{l}
\kappa_{x} \\
\kappa_{y} \\
\kappa_{x y}
\end{array}\right]=[D]^{-1}\left[\begin{array}{l}
0 \\
0 \\
M_{x y}
\end{array}\right]} \\
& \kappa_{x y}=D_{66}{ }^{*} M_{x y}
\end{aligned}
$$

where $D_{i j}{ }^{*}$ are the elements of $[D]^{-1}$ matrix. The twist of the cross section in a beam is defined by Kollár et al. [101]:

$$
\psi=\frac{\partial w_{0}}{\partial y}
$$

and the rate of twist $\xi$ is:

$$
\xi=\frac{\partial \psi}{\partial x}
$$

Combining equations (3.13) and (3.21), the out-of-plane curvature of the beam can be written in the form of:

$$
\kappa_{x y}=-2 \frac{\partial \frac{\partial w_{0}}{\partial y}}{\partial x}=-2 \frac{\partial \psi}{\partial x}
$$


Thus, we have:

$$
\xi=-\frac{1}{2} \kappa_{x y}
$$

By combining equations (3.19), (3.20), and above (3.24), one can obtain the rate of twist of a rectangular composite beam in terms of the applied twisting moment:

$$
\xi=\frac{D_{66}^{*}}{4 b} \bar{T}
$$

Comparing (3.24) with the rate of twist of an isotropic beam (i.e. $\xi=\frac{\bar{T}}{G J}$ ), the torsional rigidity of a symmetric composite beam with a rectangular cross section and sufficiently high width to thickness ratio $(>10)$ is:

$$
G J=\frac{4 b}{D_{66}^{*}}
$$

\subsubsection{Optimization process}

A sound approach to the design of an optimized composite structure for bone plate applications begins with identification of all of the factors that could affect the rigidity and strength of the structure. The most important input parameters involved in the structural rigidity of the bone plate are the type of materials, number of materials, the number of plies made of each material, ply thickness, and the stacking sequence of the laminate. The total thickness of the plate was made to be equivalent to that of a conventional metallic plate $(5.6 \mathrm{~mm})$, and the thickness of each ply was assumed to be approximately $0.2 \mathrm{~mm}$ in the cured structure [81], resulting in 28 plies in total. Carbon fibre/epoxy was used at the outermost layers to obtain superior strength and to increase bending and torsional rigidity $[64,81]$. A flax fibre/epoxy core was used to allow for the potential to obtain the desired axial and bending rigidity. The only configurations considered were those which:

1- were symmetric about the mid-surface - eliminates the bending-stretching coupling which causes an undesirable warping due to in-plane working loads, and temperature changes during manufacturing processes [102]. 
2- were balanced - this requirement indicates that angled plies (those at any angle other than $0^{\circ}$ and $90^{\circ}$ ) should occur only in balanced pairs, which uncouples in-plane normal and shear behaviour. Symmetric and balanced laminates also maximize buckling strength [103].

3- contained $\mp 45^{\circ}$ plies at their outer surfaces — maximizes buckling strength and keeps the primary load carrying plies $\left(0^{\circ}\right)$ away from the outer surfaces [103].

4- had greater than $10 \%$ of $0^{\circ}$ and $90^{\circ}$ plies - avoids excessively high thermal coefficients of expansion, which can cause undesirable warping/curvature during the manufacturing process [103].

5- had $60 \%$ or less of plies in any direction - prevents the laminate from splitting parallel to the principal loading axis at screw holes [103].

6- had at least six $90^{\circ}$ plies at the mid-surface - increases transverse rigidity while minimally affecting the bending and torsional rigidity.

All candidates which did not meet requirements 1-6 were excluded from the set. Consequently, the only design variables considered in the current optimization process were the number of lateral CF/epoxy plies and the stacking sequence of the laminate.

The ultimate goal of the current optimization process was to maximize the bending and torsional rigidity of the bone plate while keeping it axially flexible, thereby minimizing the axial rigidity. As a result of the symmetric laminate assumption, only 14 plies were taken into account. Four different angles were considered as possible orientations for each ply (i.e. $0,-45^{\circ}, 45^{\circ}$, and $90^{\circ}$ ). The number of carbon fibres $\left(n_{c}\right)$ ranged from 2 to 28 (taking only even numbers due to symmetry), resulting in 14 configurations. Considering all combinations in the number of CF/epoxy plies and ply orientations resulted in $14 \times 4^{14}$ possible configurations. Out of all of these conceivable configurations, those which did not satisfy the above-mentioned criteria were excluded from the set of configurations, yielding a total of 279,990 configurations. A code developed in MATLAB (The Mathworks Inc., MA, USA) (included in Appendix A1) was used to obtain the axial, bending, and torsional rigidity for the remaining configurations using (3.15), and (3.25) respectively.

There is no data in the literature regarding the required axial and torsional rigidity of a bone fracture plate. Bending rigidity is reported to be the leading factor that defines the fracture stability 
[87]. The minimum bending rigidity required for proper healing without significant pain in long bones was reported in a clinical trial to be 2 N.m per degree [84]. Considering their methodological setup, this is equivalent to a bending rigidity of $8 \mathrm{~N} . \mathrm{m}^{2}$. Out of all of the existing combinations, those resulting in a laminate bending rigidity lower than $8 \mathrm{~N} . \mathrm{m}^{2}$ were excluded from the analysed set. The remaining 199,905 configurations were then categorized into intervals of $1 N . m^{2}$ based on their bending rigidity. Of the configurations in each interval, the one with the highest Stiffness Efficiency Factor (SEF) was chosen. This factor is defined by:

$$
S E F_{i}=0.5\left(\frac{E A_{\min }}{E A_{i}}\right)+0.5\left(\frac{G J_{i}}{G J_{\max }}\right)
$$

where $E A_{i}$ and $G J_{i}$ are the axial and torsional rigidity of the $i^{t h}$ configuration respectively, and $E A_{\min }$ and $G J_{\max }$ represent the minimum axial rigidity and the maximum torsional rigidity amongst all configurations respectively. The $S E F$ explains how close each configuration in a given interval for bending rigidity is to the ideal case, in which the laminate is rigid in torsion while axially flexible. The 0.5 coefficients represent the weight of minimizing axial rigidity and maximizing torsional rigidity, which were assumed to be equal in this study. It is worth noting that they could be adjusted to increase or decrease the weight of each rigidity (e.g. when attaining a high torsional rigidity is more critical). Considering the aforementioned selection procedure, the number of configurations which passed to the second round were 13. For comparison purposes, a configuration that was recently proposed in the literature, and which used the same fabrics [81] and a conventional titanium alloy (Ti-6AL-4V) metallic bone plate, was also examined, leading to a total of 15 configurations.

\subsubsection{Finite Element Analysis}

\section{Development of computer-aided design (CAD) models}

A computer-aided design (CAD) model of the synthetic femur was created using a large left fourth generation composite femur (Model 3406, Sawbones, Vashon, WA) which had CT images obtained every $0.5 \mathrm{~mm}$ along the length. This artificial femur's geometry, in turn, was originally developed by the manufacturer based on an adult, male, cadaveric, human femur. The 3D model of the femur was imported into SolidWorks software (SolidWorks Corp., Dassault Systèmes, 
Concord, MA, USA). To virtually model a total hip arthroplasty (THA), the femoral head was removed by cutting at an angle of $55^{\circ}$ with respect to the femoral axial plane along the greater trochanter. A portion of the proximal cancellous bone was also removed to fit a hip implant. A Stryker Exeter hip stem (size 2, offset $37.5 \mathrm{~mm}$ ) whose geometry was obtained through laser scanning the implant's outer surface using a NextEngine 3D scanner (NextEngine, Inc., Santa Monica, CA, USA) [104], was inserted into the proximal end of the femur, and the space between the hip stem and the bone was filled with surgical Simplex P bone cement (Stryker, Mahwah, NJ, USA). To evaluate the bone plate configurations at pre- and post-healing conditions, three stages were considered: immediately post-operative (IPO), post-operative (PO), and healed bone (HB).

In the IPO stage, Vancouver B1 fractures at three different angles were considered. These included a transverse fracture, a $60^{\circ}$ proximal medial to distal lateral (PMDL) fracture, and a $60^{\circ}$ proximal lateral to distal medial (PLDM) oblique fracture. These angles were measured with respect to the axial plane of the femur. The fracture was modeled as a 4-mm bone gap at mid-shaft, and fixed with an eight-hole plate (246 $\mathrm{mm}$ long) on the lateral side. The plate was modeled as a shell to ensure it would be capable of being used as a composite laminate in later simulations. At this stage, $30 \%$ of the hip joint and muscle forces were applied to the bone, simulating partial weight bearing post-operatively. Locking screws $(4.5 \mathrm{~mm}$ dia.) were modeled as solid bodies and positioned at proximal and distal sides of the fracture. In oblique fractures, the screw passing through the fracture surface was removed, as done clinically.

To simulate the post-operative (PO) stage, which represents 4-6 weeks following plate attachment and exhibits callus formation at the fracture gap, the CAD model of the IPO stage was used with a new body located in the fracture gap to model the callus, whose properties were averaged for central, adjacent, and peripheral callus from the literature [105] and were included in Table 3-1.

In the healed bone (HB) stage, where bone continuity was assumed to be recovered, the gap was filled with cortical bone.

\section{Meshing, material properties and contact modeling}

Assemblies were imported into ANSYS Workbench 15.0 software (ANSYS Inc., Canonsburg, PA, USA) to generate finite element (FE) models (Figure 3-2). The bone, hip implant, bone cement and screws were meshed using quadratic tetrahedral elements, whereas the plate was meshed using 
quadrilateral 4-node elements. Surface-to-surface contact elements (CONTA174 and TARGE170) were used to mesh all interfaces. Mesh sensitivity was performed using the "relevance" option in ANSYS Workbench software, with a range between -100, indicating a very coarse mesh, and 100, corresponding to an extremely fine mesh. A value of $75 \%$ was chosen, as it was found that further increasing the relevance resulted in a less than $1 \%$ change in the maximum displacement and average nodal strain values in the model. The number of body elements after convergence was 313,949 and the number of contact elements was 171,784 , leading to 485,733 elements in total in the FE model.

Contact between the following contact pairs was set to "bonded": cancellous bone-cortical bone, screws-bone, screws-plate, hip stem-bone cement and bone cement-cancellous bone. However, contact between the plate and the bone was considered to be no-separation, which allows for sliding of the touching surfaces [91, 104]. The bodies were assigned linear elastic properties as tabulated in Table 1. The screws and the hip implant were made from 316L surgical-grade stainless steel and CoCrMo (cobalt-chrome-molybdenum), respectively. The metallic bone plate, made of a titanium alloy (Ti-6Al-4V), was assigned a thickness of $5.6 \mathrm{~mm}$. ANSYS Composite PrepPost module was employed to assign the stacking sequence of the laminates in the composite bone plates selected in section 3.2.2. 


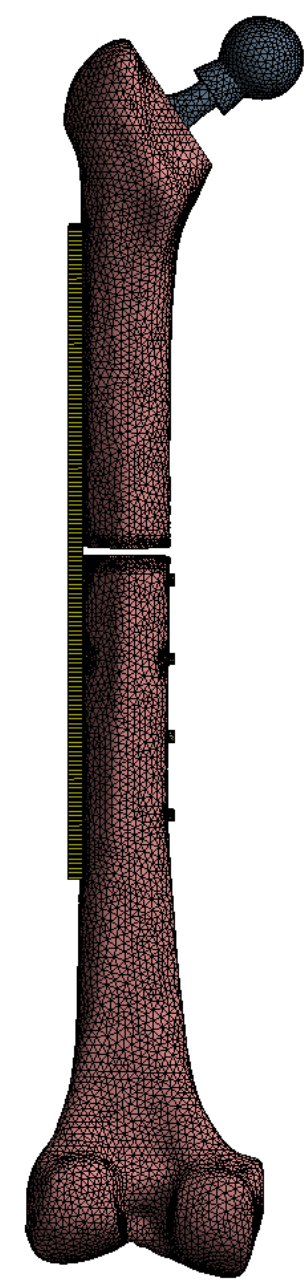

(a)

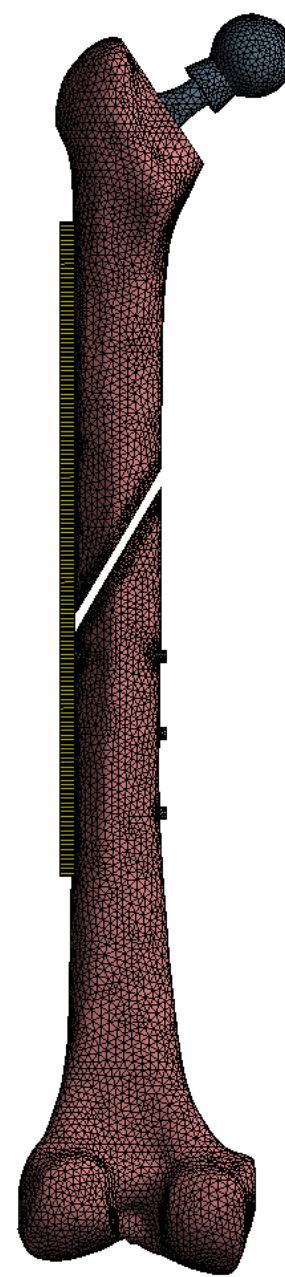

(b)

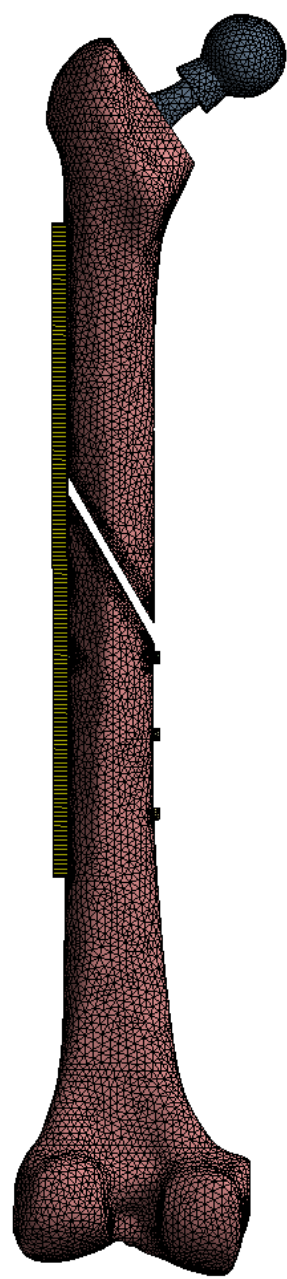

(c)

Figure 3-2. Finite element models of a femur with a transverse (a), proximal medial to distal lateral (PMDL) oblique (b), and proximal lateral to distal medial (PLDM) oblique (c) Vancouver B1 periprosthetic fracture, which was fixed with an eight-hole bone plate. 
Table 3-1. Linear elastic material properties used in this study [104-109]

$$
\begin{array}{cccccccccc}
E_{X} & E_{Y} & G_{X Y} & v_{X Y} & v_{Y Z} & \sigma_{X U} & \sigma_{Y U} & \sigma_{X U}^{\prime} & \sigma_{Y U}^{\prime} & \tau_{X Y U} \\
(G P a) & (G P a) & (G P a) & & & (M P a) & (M P a) & (M P a) & (M P a) & (M P a)
\end{array}
$$

\begin{tabular}{c|c|c|c|c|c|c|c|c|c|c|c|}
\hline $\begin{array}{c}\text { Composite } \\
\text { Prepregs }\end{array}$ & Flax/epoxy* & 35 & 4.4 & 2.1 & 0.3 & 0.4 & 330 & 31 & -150 & -80 & 79 \\
\hline \multicolumn{2}{|c|}{ CF/epoxy* } & 121 & 8.6 & 4.7 & 0.27 & 0.4 & 2231 & 29 & - & -100 & 60 \\
\hline \multicolumn{2}{|c|}{ Ti-6AL-4V } & 113.8 & - & - & 0.34 & - & 950 & - & - & - & - \\
\hline 316L stainless steel & 193 & - & - & 0.3 & - & 235 & - & - & - & - \\
\hline \multicolumn{2}{c|}{ CoCrMo } & 210 & - & - & 0.31 & - & 872 & - & - & - & - \\
\hline \multicolumn{2}{c|}{ Bone Cement } & 2.45 & - & - & 0.38 & - & 62 & - & - & - & - \\
\hline \multicolumn{2}{c}{ Callus } & 0.1 & - & - & 0.35 & - & 23 & - & - & - & - \\
\hline \multirow{2}{*}{ Bone } & Cortical & 16.7 & - & - & 0.26 & - & 106 & - & - & - & - \\
\hline & Trabecular & 0.155 & - & - & 0.3 & - & 6 & - & - & - & - \\
\hline
\end{tabular}

*The layers were made of unidirectional (UD) prepreg flax and carbon sheets, with their $X$-axis aligned with the long axis of the bone plate.

\section{Gait loads and boundary conditions}

The distal epiphysis was constrained in all directions to avoid rigid body motion. The present thesis employed a comprehensive load case at $45 \%$ gait cycle presented by Duda et al. [110], [111] and simplified by Bitsakos et al. [112]. It included contributions from five major muscles (i.e. $\mathrm{m}$. gluteus maximus, m. gluteus medius, m. gluteus minimus, m. psaos, and m. abductor magnus) and the hip joint force. This reduced muscle system was selected because previous studies $([111,112])$ have shown that the aforementioned muscles have the most significant effect on the internal loads of the femur at $45 \%$ of the gait cycle due to their magnitudes and directions. The m. abductor magnus and $\mathrm{m}$. abductor mimimus were not considered in this study as they have negligible effects at this instant of the gait cycle. The muscle attachment points obtained by Duda et al. [111] and scaled for use on the $3^{\text {rd }}$ generation composite femur by Cheung et al. [5] were employed, as there is no geometrical difference between the $3^{\text {rd }}$ generation and neration composite femurs. Force components, tabulated in Table 3-2, were applied to the femur as shown in Figure 3-3. 


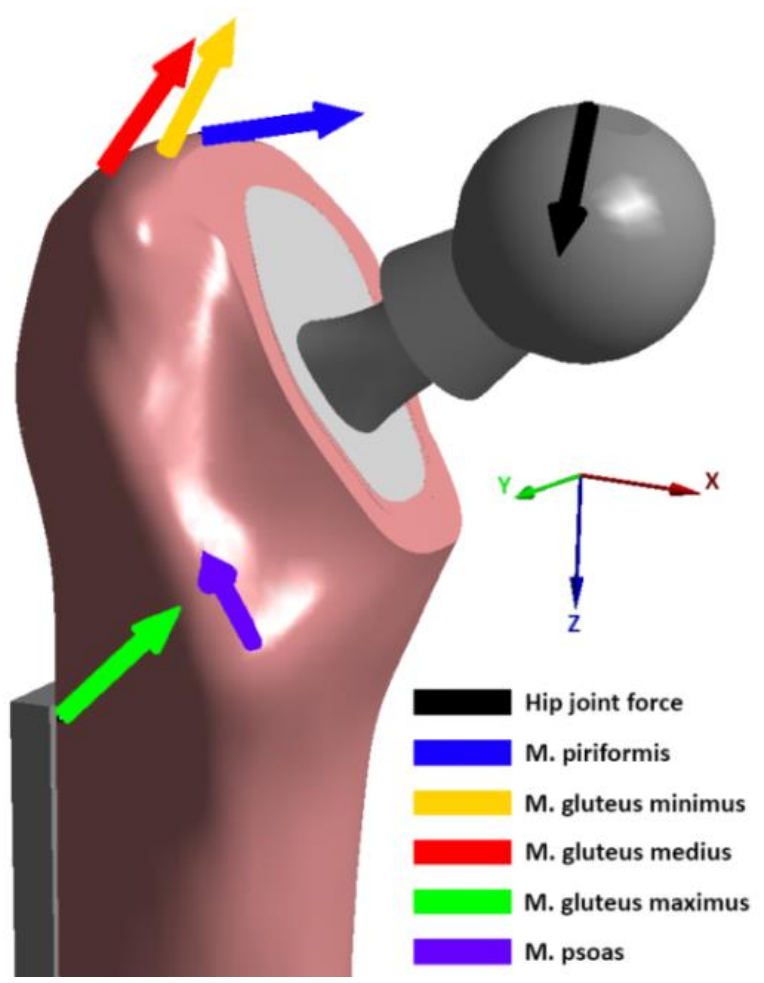

Figure 3-3. Diagram showing the five major muscles and hip joint forces applied to the femur. The coordinate system used for load application is also shown. Note that the $z$-axis aligns with the femoral shaft axis and the $x$-axis lies in the femoral coronal plane.

Table 3-2. Force components for muscle and joint reaction forces. Muscle force data taken from Duda et al. [110] and [112].

\begin{tabular}{c|c|c|c|}
\multirow{2}{*}{ Muscle name } & \multicolumn{3}{c}{ Force components $(\boldsymbol{N})$} \\
\cline { 2 - 4 } & $X$ & $Y$ & $Z$ \\
\hline Hip joint force & -613.7 & -219.3 & 2868.7 \\
\hline Piriformis & 110.5 & -70.1 & -22.4 \\
\hline Gluteus minimus & 25.4 & -0.7 & -51.6 \\
\hline Gluteus medius & 63.7 & -28.9 & -113.3 \\
\hline Gluteus maximus & 172.3 & -105 & -203.8 \\
\hline Psoas & 3.6 & 160.6 & -158.5 \\
\hline
\end{tabular}




\section{Output parameters}

In order to measure fracture stability and evaluate the healing process, seven parameters were employed as follows:

- Axial movement - relative axial movement of the fracture ends, which is one of the factors defining fracture stability has been previously employed [29, 30, 82, 85]. Moderate axial movements (less than $2 \mathrm{~mm}$ ) in diaphyseal fractures have been shown to be advantageous in stimulating periosteal callus formation [29], while excessive axial movement can degrade healing [85]. Therefore, axial movement at the fracture site was selected as an output parameter when evaluating fracture stability in the IPO stage, and was defined by the relative motions (normal to the fracture plane) between the two nodes at each fracture end located farthest from the plate (Figure 3-4).

- Shear movement - high levels of shear movement at the fracture site result in reduced stability and are believed to be the leading factor in producing a predominance of fibrous tissue at the fracture site, resulting in delayed union or non-union [9, 29]. Shear movement at the fracture site was therefore selected as an output parameter to indicate fracture stability in the IPO stage. It was defined as the relative motions (tangent to the fracture plane) between the two nodes at each fracture end located farthest from the plate (Figure 3-4).

- Inverse safety factor (ISF) — the failure of the composite bone plate is an important factor to consider, as failure in the laminate can result in difficult-to-manage complications. The strength of materials can be predicted based on different failure criteria. The safety factor indicates a margin to failure that, when multiplied by the applied load, gives the failure load. The inverse safety factor (ISF) is more commonly used in practical situations, with non-critical values ranging from $0-1$, while a value greater than 1 is considered critical. In the present study, the ISF reported is based on the Tsai-Hill failure criterion for composite laminate and the maximum equivalent stress for titanium alloys. The ISF was obtained in the IPO stage where there is a fracture gap and the plate is under excessive bending stress.

- Compressive normal force - compressive normal force at the fracture surface was used as an output parameter in the PO stage, as it stimulates proliferation of the periosteal callus and so has a beneficial effect on the healing rate $[29,48,113]$. 
- Contact bending moment - local strain distribution at the fracture site provides the mechano-biological signal for the regulation of fracture healing and causes the cellular reaction [29]. Although uniform compressive stress at the fracture site is ideal for fracture healing as it encourages even enchondral bone formation, there is always a nonuniform pattern of stress at fracture due to bending moments. To investigate the level of normal stress uniformity at the fracture site, the bending moment resulting from bone-on-bone contact was used as an output parameter in the PO stage. The moment was calculated using the normal contact stresses at the fracture surface. A zero contact moment corresponded to purely even compression, whereas a large bending moment indicated an uneven pattern of normal stress $[92,114]$.

- Mean stress - to assess the changes in the stress pattern imposed by the external implant (i.e. load sharing), the mean value for the nodal von-Mises stress in the femoral shaft after healing (in the HB stage) was selected to be a parameter. Von-Mises stress was chosen because it not only represents an accurate state of stress in each node, but it is also always positive. Negative nodal results, as in the case of maximum principal stress or strain, could bias the average results.

- Maximum deformation - several studies concerning bone plate design have introduced the rigidity of the bone-implant structure as one of the primary factors involved in assessing fracture healing and the level of stress shielding caused by the implant [90, 91]. The maximum deformation of the structure in the HB stage was obtained and compared to that of the intact bone to evaluate the effect of implant rigidity on the structural rigidity [114]. 


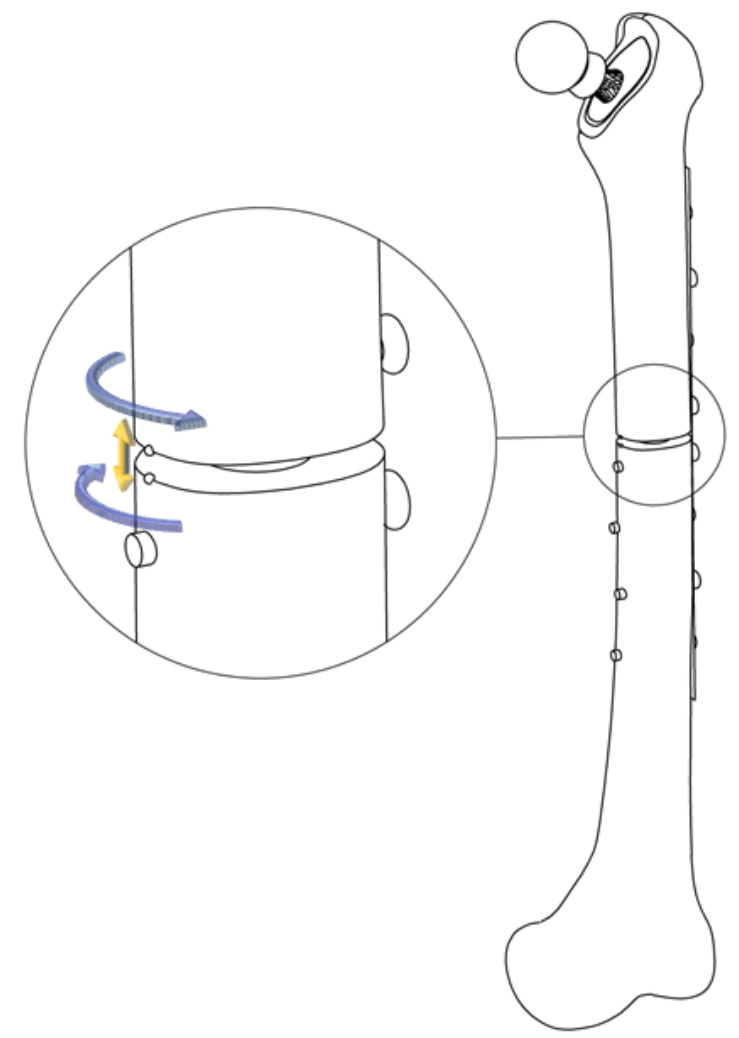

Figure 3-4. Schematic figure showing axial and shear movement measurements at the fracture site

\subsection{Results}

\subsubsection{The optimal configurations}

Axial, bending, and torsional rigidity for the configuration with the highest SEF in each interval of bending rigidity have been tabulated in Table 3-3. A configuration used for CF/flax/epoxy in the literature [81], and a Ti bone plate were also included in the table for the comparison purposes, resulting in 15 configurations in total $(\mathrm{C} 1-\mathrm{C} 15)$. 
Table 3-3. Optimal configurations in the intervals of bending rigidity. EA: Axial rigidity, EI:

Bending rigidity, GJ: Torsional rigidity of the plate

\begin{tabular}{|c|c|c|c|c|c|}
\hline Conf. & Stacking Sequence & $\begin{array}{c}\text { EI } \\
\left(N . m^{2}\right)\end{array}$ & $\begin{array}{c}\text { EA } \\
(M N)\end{array}$ & $\begin{array}{c}\text { GJ } \\
\left(N . m^{2}\right)\end{array}$ & SEF $^{*}$ \\
\hline C1 [81] & {$\left[0_{C} / 0_{8 F}\right]_{S}$} & 4.0 & 2.7 & 0.76 & - \\
\hline $\mathrm{C} 2$ & {$\left[\mp 45_{2} / 45 / 0 /-45 / 0 / 45 / 0 /-45 / 90_{3}\right]_{S}$} & 8.1 & 4.2 & 27.1 & 0.49 \\
\hline $\mathrm{C3}$ & {$\left[\mp 45 / 0 / \pm 45_{2} / \mp 45 / 0_{2} / 90_{3}\right]_{S}$} & 9.0 & 4.2 & 26.2 & 0.47 \\
\hline C4 & {$\left[\mp 45 / 0 / \mp 45 / 45 / 0 / \mp 45 /-45 / 0 / 90_{3}\right]_{S}$} & 10.0 & 4.2 & 25.1 & 0.46 \\
\hline C5 & {$\left[\mp 45 / 0 / \pm 45 / 0 / \pm 45 / 0 / \mp 45 / 90_{3}\right]_{S}$} & 11.0 & 4.2 & 24.2 & 0.44 \\
\hline C6 & {$\left[\mp 45 / 0_{2} / \pm 45_{2} / 0 / \mp 45 / 90_{3}\right]_{S}$} & 12.1 & 4.2 & 23.1 & 0.42 \\
\hline C7 & {$\left[\mp 45 / 0_{2} / 45 / 0 /-45_{2} / 45_{2} /-45 / 90_{3}\right]_{S}$} & 13.3 & 4.2 & 21.8 & 0.40 \\
\hline $\mathrm{C8}$ & {$\left[\mp 45 / 0_{2} / 45 / 0 /-45_{2} / 45 / 0 / 90_{4}\right]_{S}$} & 14.0 & 4.8 & 20.9 & 0.38 \\
\hline C9 & {$\left[\mp 45 / 0_{2} / 45 / 0_{2} /-45_{2} / 45 / 90_{4}\right]_{S}$} & 15.1 & 4.8 & 19.8 & 0.36 \\
\hline C10 & {$\left[\mp 45 / 0_{4} / \pm 45_{2} / 90_{4}\right]_{S}$} & 16.0 & 4.8 & 18.9 & 0.35 \\
\hline C11 & {$\left[\mp 45 / 0_{4} / 45 / 0 /-45_{2} / 45 / 90_{3}\right]_{S}$} & 17.1 & 5.5 & 18.0 & 0.33 \\
\hline $\mathrm{C} 12$ & {$\left[\mp 45 / 0_{4} / 45 / 0 /-45 / 0_{2} / 90_{3}\right]_{S}$} & 18.1 & 6.8 & 17.0 & 0.31 \\
\hline C13 & {$\left[\mp 45 / 0_{6} / \pm 45 / 0 / 90_{3}\right]_{S}$} & 19.1 & 6.8 & 16.0 & 0.29 \\
\hline C14 & {$\left[\mp 45 / 0_{8} / 90_{4}\right]_{S}$} & 20.3 & 7.3 & 14.5 & 0.26 \\
\hline C15 & Ti-6AL-4V & 28.3 & 10.8 & 42.2 & - \\
\hline
\end{tabular}

*SEF is calculated using Eq. (3.26).

\subsubsection{FEA results}

\section{IPO stage}

Immediately post-operative results were measured at the level of the fracture gap. Axial and shear movements between fracture fragments for a Vancouver B1 fracture fixed with composite and metallic bone plate configurations (C1-C15) is shown in Figure 3-5. Figure 3-6a shows the inverse 
safety factor (ISF) based on the Tsai-Hill failure criterion for the composite plate and the maximum equivalent stress failure criterion for the metallic plate. A typical distribution of the ISF in a composite configuration (C7) for transverse and oblique fractures has also been illustrated in Figure 3-6 b-d.
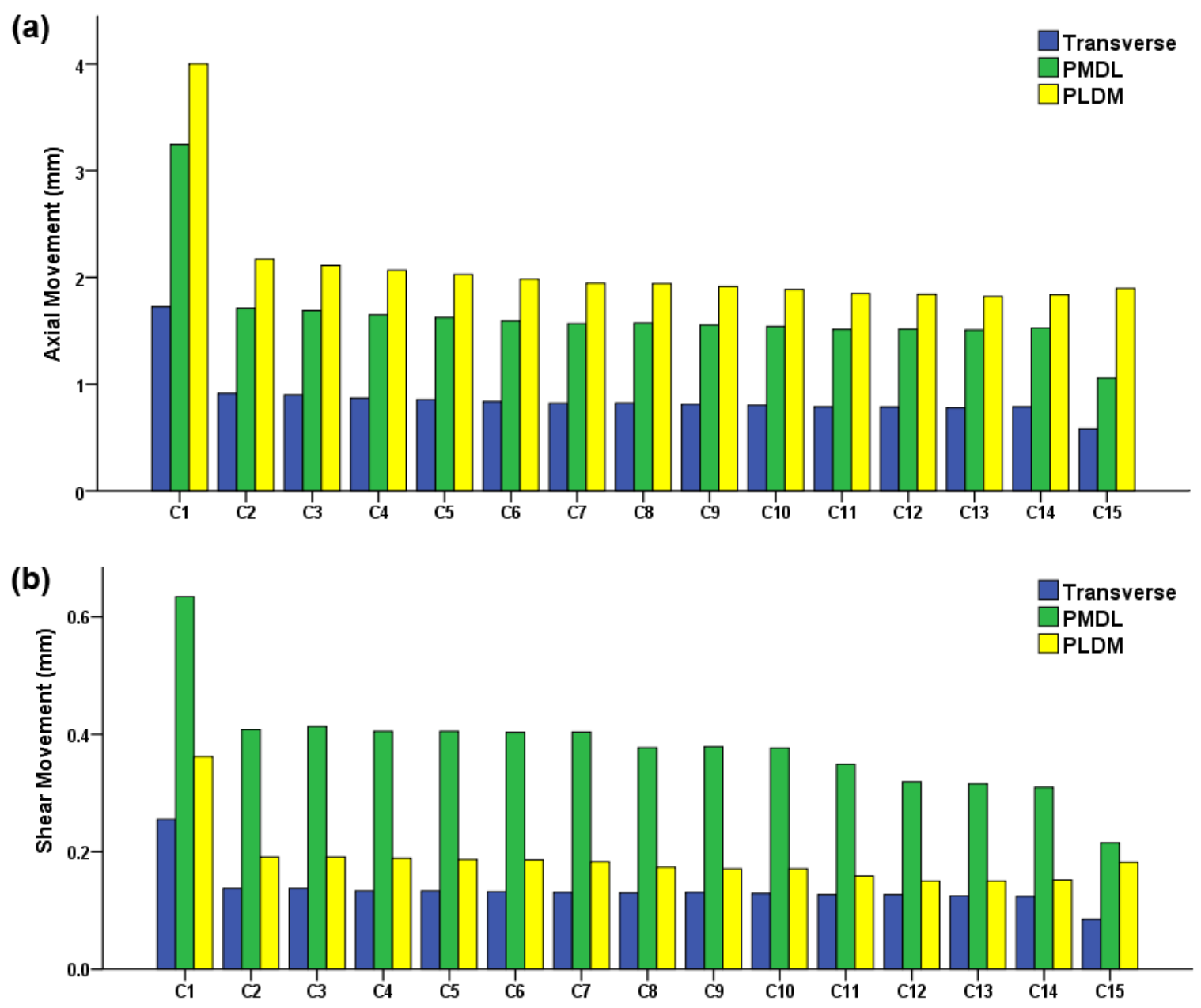

Figure 3-5. Axial (a) and shear movement (b) between fracture fragments in transverse and oblique Vancouver B1 fracture fixed with different composite bone plate configurations (C1$\mathrm{C} 14)$ as well as the metallic plate (C15). 


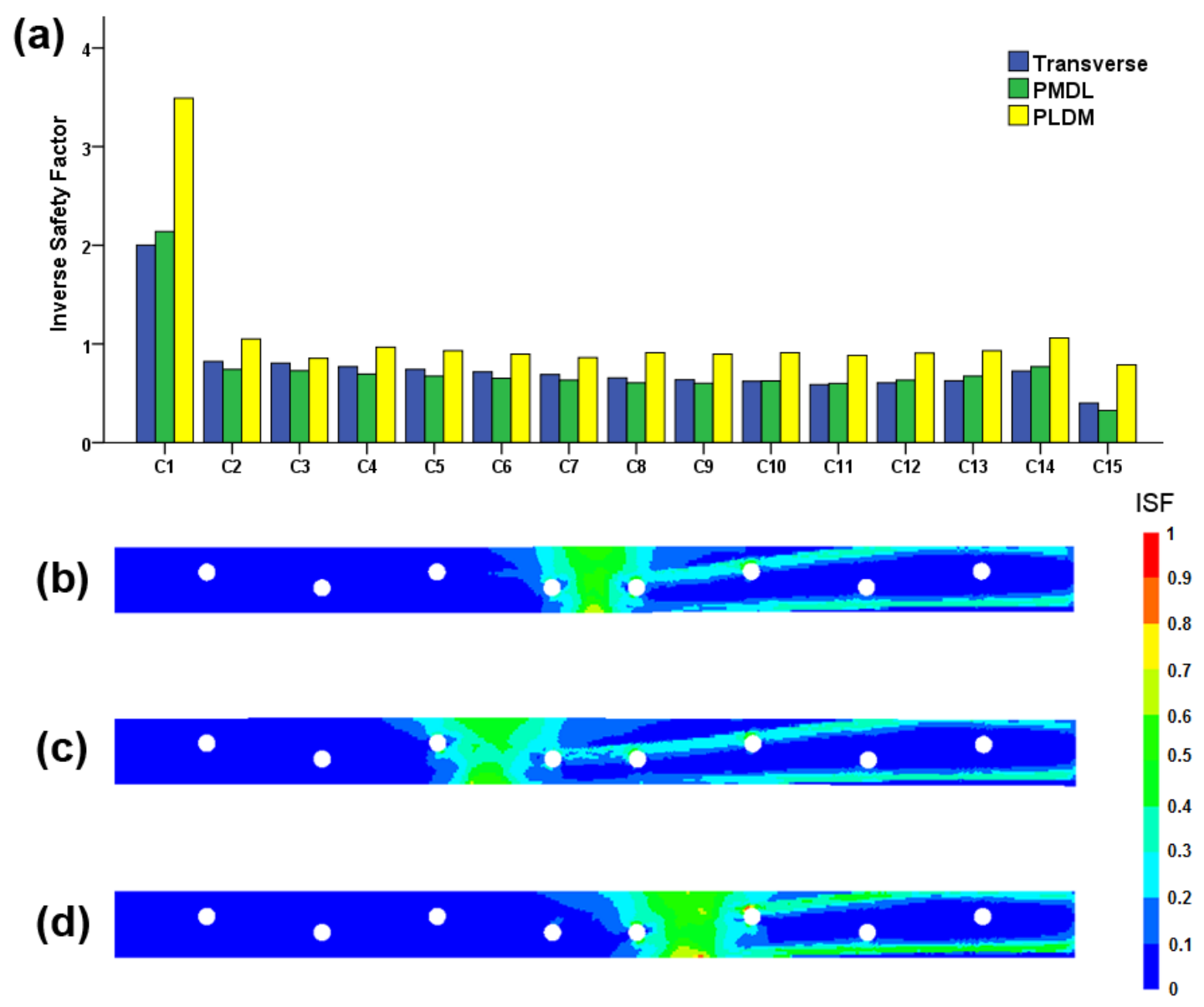

Figure 3-6. The inverse safety factor (ISF) for composite bone plate configurations (C1-C14) (based on Tsai-Hill failure criterion) and the metallic bone plate (C15) (based on maximum equivalent stress failure criterion) for transverse and oblique Vancouver B1 fractures (a). The distribution of the ISF in a typical composite bone plate configuration (C7) for transverse (b), PMDL oblique (c), and PLDM oblique (d) fractures.

\section{PO stage}

Post-operative results, simulating 4 to 6 weeks following fixation, are depicted in Figure 3-7. They include the compressive normal force and the contact bending moment at the fracture site. 

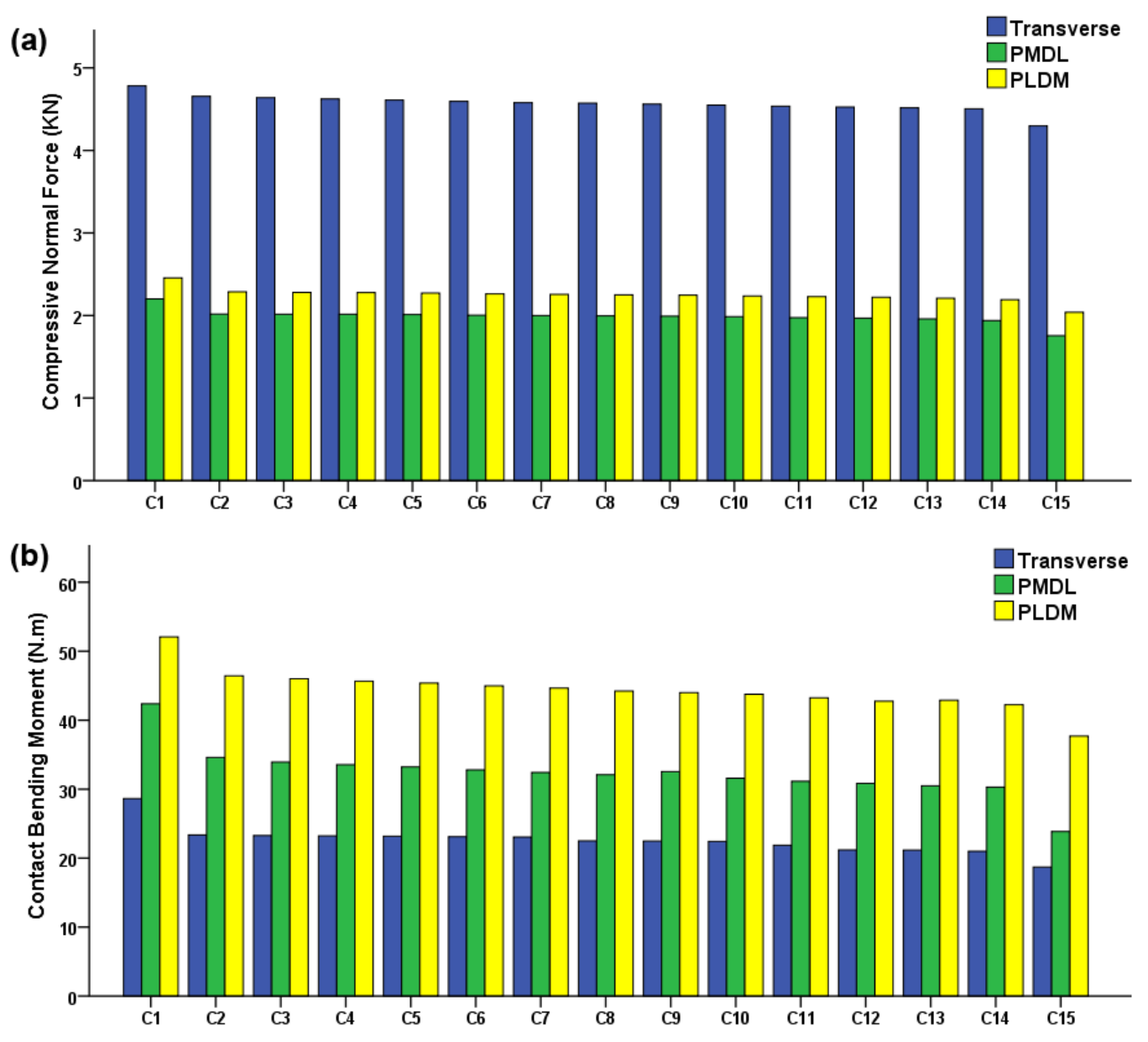

Figure 3-7. Compressive normal force (a) and contact bending moment (b) at a transverse and oblique Vancouver B1 fracture site fixed with composite $(\mathrm{C} 1-\mathrm{C} 14)$ and metallic $(\mathrm{C} 15)$ bone plates.

\section{HB stage}

The average equivalent bone stresses and the maximum deformation of the bone-implant construct obtained in the healed-bone (HB) stage were compared to those in an intact bone (Figure 3-8). Figure 3-9 shows the strain distribution in an intact femur in addition to the same femur implanted with CF/epoxy and $\mathrm{Ti}$ bone plates. 


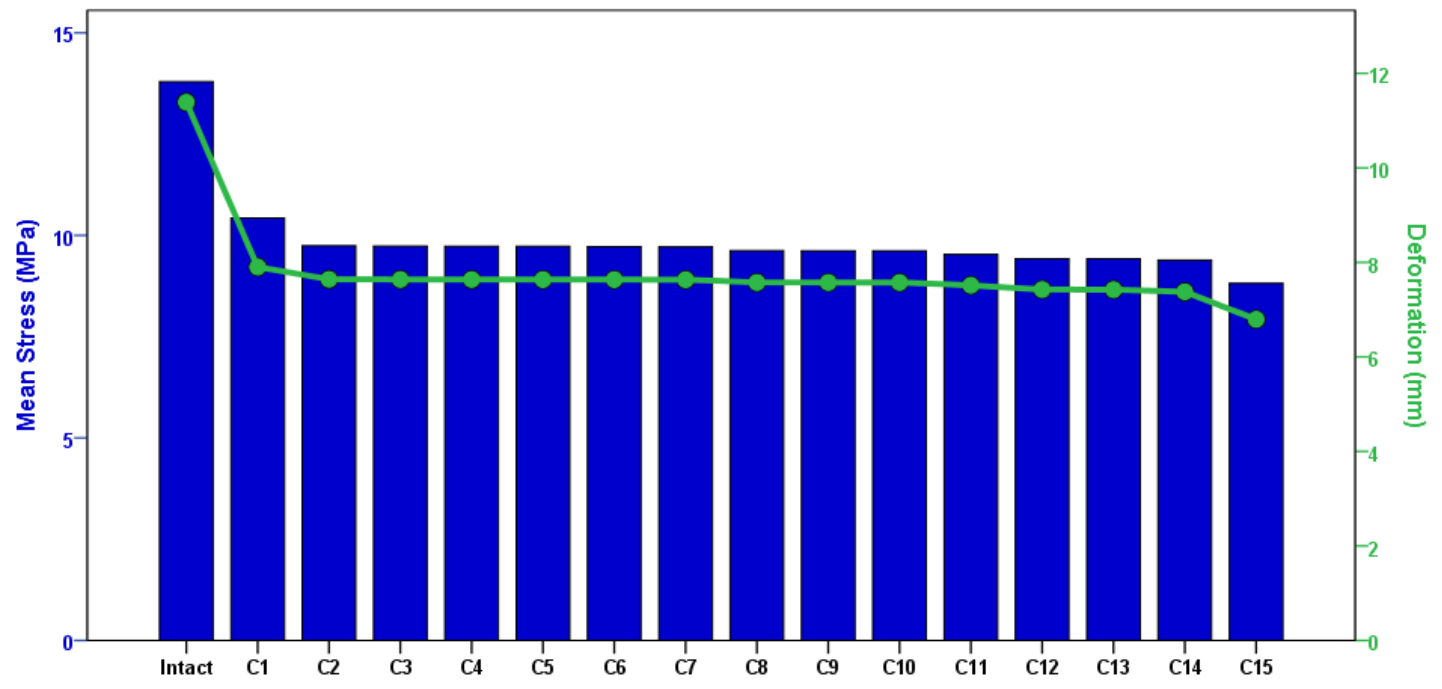

Figure 3-8. Mean nodal von Mises stress and the maximum deformation in the intact femur, the healed femur fixed with the composite plate configurations (C1-C14), and the healed femur fixed with the metallic plate $(\mathrm{C} 15)$. 


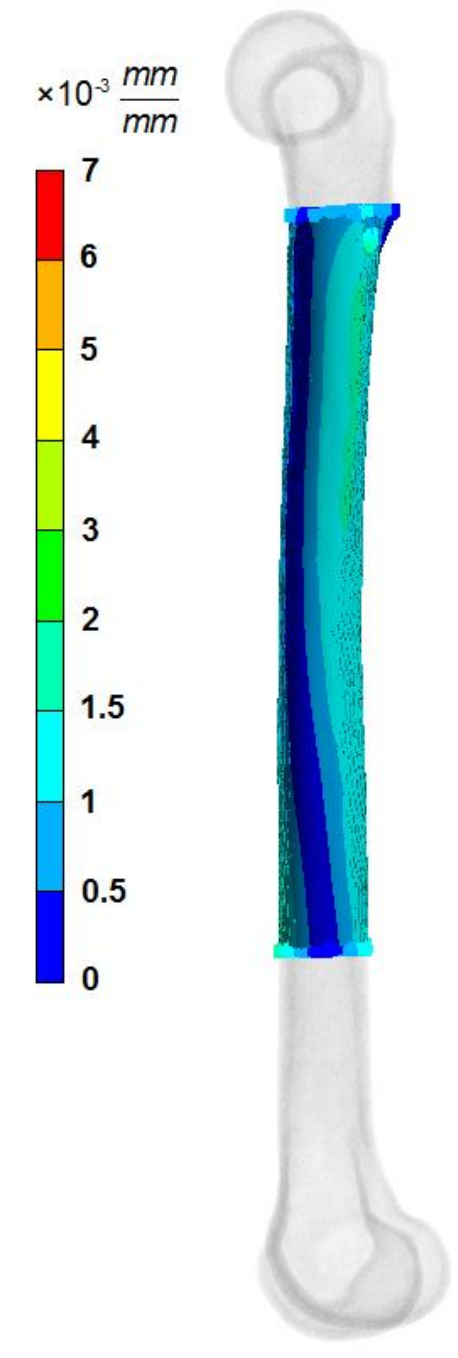

(a)

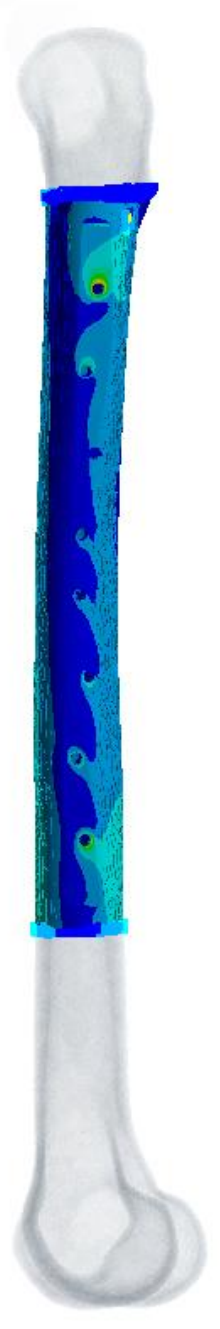

(b)

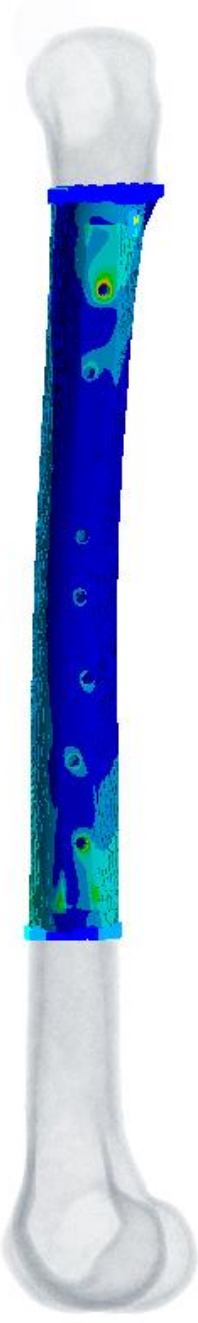

(c)

Figure 3-9. Strain distribution in the intact femur (a), the healed femur implanted with CF/epoxy plate (b) and the healed femur implanted with Ti plate (c)

\section{Validating FEA with experiments}

For the finite element results to be credible, it is crucial that they are verified against experimental data for at least one configuration [115]. FE models of the IPO and HB stages were selected to be verified against infrared (IR) thermography camera measurements, as outlined in a previous study [91]. To replicate the test setup, a compressive force of $750 \mathrm{~N}$ was applied to the hip prosthesis 
head in the femoral coronal plane at an angle of $15^{\circ}$ to the femoral axis. The sum of the principal stresses obtained from the FE models at each of 4 points on the femoral surface were compared to equivalent measurements found using an IR thermography camera, as reported in Bagheri et al. [91]. As shown in Figure 3-10, good correlations (IPO stage: $R^{2}=0.9411$, slope $=1.0347$; HB stage: $R^{2}=0.9851$, slope $\left.=0.9465\right)$ were found between the $\mathrm{FE}$ values and experimental measurements.
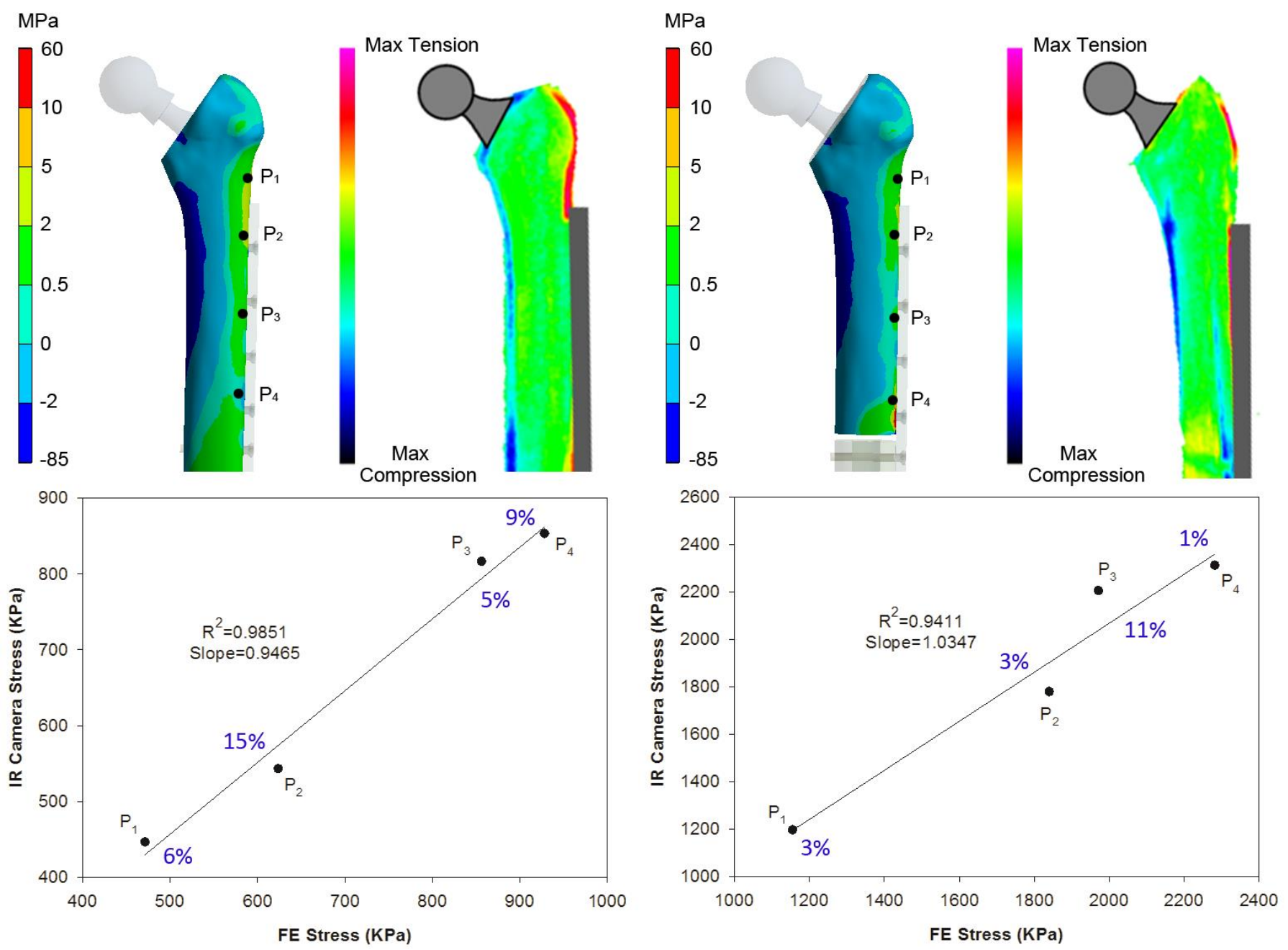

Figure 3-10.Validation of FE results against IR thermography camera measurements by Bagheri et al. [91]. Values show the sum of principal stresses on the bone surface. (a) and (d) show FE results in healed and fractured femur respectively, (b) and (e) shows IR thermography camera measurements in aforementioned configurations (adopted with permission), (c) and (f) illustrate the graphs with slope and $\mathrm{R}^{2}$. The values in blue show the percentage error for each point. 


\subsection{Discussion}

\subsubsection{General findings}

Reducing the rigidity of the fracture fixation can favourably increase the compressive normal stress at the fracture site, which can in turn boost fracture healing $[48,82,116]$. However, stability of the fracture is always a concern with a flexible fracture fixation [30,82]. One school of thought, referred to as "selective stress shielding," suggests that the fixation device should resist detrimental loads (i.e. shear and bending), yet allow for some compression of the fracture ends [48, 85]. In transverse fractures, this is equivalent to reducing the axial rigidity of the implant while keeping it rigid in bending and torsion. Conversely, in oblique fractures, this can be achieved through altering both the axial and bending rigidity. Designing a bone plate with such capabilities is technically impractical with conventional metallic alloys. Fibre reinforced composite bone plates have been introduced because of their high strength, corrosion and fatigue resistance, as well as their capability to obtain desired rigidity and strength values in different directions [11, 81, 94]. Although several studies have investigated the use of fibre reinforced composite bone plates for fixation of diaphyseal fractures $[58,65,66,79,81]$, none have considered the optimization of such implants to fulfill the requirements of an efficient fixation.

The composite concept made of CF/flax/epoxy was optimized to obtain the lowest axial rigidity with the highest bending and torsional rigidity. The optimization was performed by altering the contribution of carbon fibres and changing the stacking sequence of the laminate.

The findings (Table 3) revealed that, in contrast to metallic bone plates, increasing the bending rigidity in a composite plate does not necessarily result in an increase in its axial rigidity. It is interesting to note that configurations $\mathrm{C} 2-\mathrm{C} 7$ yielded the same axial rigidity but differing bending rigidity. This enables one to tailor the rigidity based on fixation requirements. When examining the optimal configurations in each set, one can see that no laminate containing a flax/epoxy core has been selected as the best configuration. This is mainly due to the low torsional rigidity of structures with a flax/epoxy core. The table also shows that the axial rigidity of the plate grows as the number of $0^{\circ}$ plies increases, but it is independent of the location of those $0^{\circ}$ plies. Conversely, the bending rigidity of the plate depends on the number of plies as well as their location. Therefore, one may place the existing $0^{\circ}$ plies in a laminate closer to the outer surface to increase the bending rigidity, while keeping the axial rigidity unchanged. When considering column GJ in Table 3, it 
can be concluded that the torsional rigidity of the plate is highly dependent upon the number of $45^{\circ}$ plies and their proximity to the outer surface. That said, use of additional $45^{\circ}$ plies favourably increases the torsional rigidity of the plate, but tends to simultaneously sacrifice the bending rigidity of the structure. C14 depicts a configuration in which sixteen $0^{\circ}$ plies and only four $45^{\circ}$ plies have been used, which resulted in the highest bending rigidity but the lowest torsional rigidity of all of the second round configurations.

It is worth mentioning that PMDL oblique fractures were found to be more critical when compared to transverse or PLDM oblique fractures, regardless of the plate configuration, as they resulted in higher shear movements and lower compressive forces at the fracture site. The plate was also more susceptible to failure due to a higher ISF. This was primarily due to the state of hip joint and muscular forces to the femur, which act to displace fragments and reduce stability in these fractures. The lower stability of oblique fractures as compared to transverse fractures was previously shown in a canine study [117].

\subsubsection{Selection of the best configuration}

The FE results revealed useful information regarding the actual performance of each configuration in the second round. In examining Figure 3-5, configurations C6-C14 resulted in an axial movement of $2 \mathrm{~mm}$ or less, reported as the allowable axial motion for periosteal callus formation by Woo et al. [85] in a canine study. Interestingly, the resultant axial movements in configurations C10-C14 are even less than those found with a Ti plate for PLDM oblique fractures. C13-C14 could be prone to failure in PLDM oblique fractures based on the Tsai-Hill failure criterion (ISF 1). Therefore, configurations C6-C12 could all be acceptable configurations, as they all resulted in an allowable degree of axial movement $(<2 \mathrm{~mm})$ and negligible shear movement $(<0.5$ $\mathrm{mm}$ ) at the fracture site, providing the required fracture immobilization. Furthermore, they did not experience failure, as established by their ISF $(<1)$. However, C7 was selected as the best configuration for the following reasons:

- It had the lowest axial (4.2 MN), and moderate bending and torsional rigidity $\left(21.8 \mathrm{~N} . \mathrm{m}^{2}\right.$ and $13.3 N . m^{2}$ respectively). 
- It had one of the lowest ISF in all of the fracture configurations when compared to other configurations, implying a sufficiently strong fixation structure for femoral diaphyseal fracture.

\subsubsection{Comparison of the best composite configuration with conventional Ti plates}

Configuration $\mathrm{C} 7$ has a bending rigidity well above $8 \mathrm{~N} \cdot \mathrm{m}^{2}$, but is almost $53 \%$ less rigid axially when compared to a conventional Ti plate. Furthermore, despite being $48 \%$ less rigid in torsion, it could provide sufficient torsional stability for all transverse and oblique fractures. The proposed configuration could increase the compressive normal force between the fracture fragments by $6.5 \%$ $(280.1 \mathrm{~N}), 14.0 \%(245.1 \mathrm{~N})$, and $10.7 \%(218.1 \mathrm{~N})$ as compared to a conventional Ti plate in transverse, PMDL oblique and PLDM oblique fractures respectively in the PO stage. In the IPO stage, it minimally affects fracture stability, as seen by the increase of $0.05-0.51 \mathrm{~mm}$ in the fragments' axial movements and 0.001-0.19 $\mathrm{mm}$ in the fragments' shear movements at different fracture angles. Examining Figure 3-7, one can observe that the contact bending moment increased by 4.4, 8.7, and 7.0 N.m for transverse, PMDL, and PLDM oblique fractures respectively, when the composite bone plate was used instead of the Ti bone plate. This indicates that although the average compressive stress increases at fracture through use of the composite plate, the distribution of compressive stress may not be as uniform as that seen when using the Ti plate.

The HB stage reflects the condition in which union has occurred and the plate is expected to minimally alter the amount and pattern of loads carried by the bone. When considering Figure 3-8, one can see that implantation of the Ti bone plate reduced the average von-Mises stress in the femoral shaft by $36 \%$ while a $29 \%$ reduction in average von-Mises stress was observed with use of the CF/epoxy bone plate. It is worth mentioning that these average values are overshadowed by the stress rise in the vicinity of the screw holes following plate implantation, and cannot accurately represent the load distribution in the healed bone. However, Figure 3-9 depicts the strain patterns in an intact femur and the healed femur with bone plates attached, and it is clearly shown that strain patterns in the femur implanted with the composite plate more closely replicate those in an intact femur. This similarity, in turn, could possibly result in less peri-implant bone loss over time as a result of bone remodelling, and could be considered another advantage of the composite bone plate. Furthermore, comparing the healed bone stage to an intact femur revealed that implantation 
of the Ti plate for a Vancouver B1 fracture caused a 40.3\% increase in the structural rigidity, while the composite nail raised the rigidity by only $32.9 \%$.

\subsubsection{Comparison with prior studies}

An increase was found in the stress levels of the bone when a composite bone plate was used to fix the fracture as opposed to a metallic plate, which is consistent with the findings of previous studies $[90,91]$. It was found that implantation of a Ti plate caused a $40.3 \%$ reduction in the maximum deformation of the bone-plate construct as compared to an intact femur, which concurs with the findings of a former study [91] in which a 39.3\% increase in overall rigidity was reported when a Vancouver B1 fracture was fixed using a Ti plate. The current results also support the rationale behind why overly flexible implants have resulted in complications during healing, as reported in the literature $[82,84]$. Excessively flexible bone plates can cause large structural deformations, particularly in the presence of a fracture gap, resulting in pain and excessive bending stress on the plate itself.

A number of studies [64, 90, 92, 93] considered unidirectional laminates for bone plate applications as they resulted in the highest bending rigidity when compared to other stacking sequences with the same laminate thickness. However, selection of UD laminates significantly reduces the torsional rigidity of the structure, which can lead to torsional instability at the fracture site. UD laminates are also inferior in supporting load components transverse to the fibre direction, and are prone to failure near the screw holes, as the laminate can easily be split in these regions [87]. The stacking sequence obtained in the current optimization process benefited from $\mp 45^{\circ}$ plies, which not only increased the buckling strength of the plate but also the torsional rigidity of the laminate, and ensured the primary load carrying plies $\left(0^{\circ}\right)$ were located away from the structure surface. Given that the minimum required bending rigidity could easily be achieved, the $\mp 45^{\circ}$ plies were used at the outermost layers to yield maximum torsional rigidity. Furthermore, the $90^{\circ}$ plies used around the midsurface increased the transverse modulus and strength and resisted laminate split around the screw holes [103]. Bagheri et al. [81] recently developed a UD sandwich structure made of CF/flax/epoxy for treating the same type of fracture as examined in this study, and showed that their design could boost the load sharing of the implant, thereby reducing its adverse stress shielding effects [91]. However, they investigated the stability of fracture by measuring the rigidity of the bone-plate construct rather than the interfragmentary 
motions at the fracture site. As depicted in Figure 3-5a, their design resulted in excessive axial movement of the fracture (>4 mm), especially in PLDM oblique Vancouver B1 fractures, which can interfere with callus formation, as reported by Woo et al. [85]. Moreover, the low torsional rigidity of their design $\left(0.76 \mathrm{~N} . \mathrm{m}^{2}\right)$ resulted in shear instability of the fracture (i.e. overriding fracture ends) (Figure 3-5b). The Tsai-Hill inverse safety factor of 2, 2.14, and 3.49 in transverse, oblique PMDL and oblique PLDM fractures indicated failure of the structure in the IPO stage of fracture healing (Figure 3-6a). The current design yielded bending and torsional rigidity more than 3 and 28 times higher, respectively, than those obtained by their design, while producing an axial rigidity twice as high as that reported. Furthermore, the current design took advantage of the tailorability of composite structures and thus optimized it for flexibility in desired directions and rigidity in others.

When comparing the findings of the current study with those reported previously concerning the design of composite bone plates, one can see that the critical role of fracture stability in designing bone plates may have been underestimated to date. Any bone plate whose rigidity is less than that of a conventional metallic one could reduce the stress shielding effect of the implant; however, one should bear in mind that the fracture stability and strength of the structure should not be overlooked when designing bone plates, as they can result in adverse effects such as excessive pain, delayed union, non-union, or failure of the fixation construct $[82,84,85]$.

Simple bending tests, which have been employed in previous studies [58, 81, 84] to evaluate the performance of fixation devices, fail to consider several critical factors in bone plate design, including the behaviour of the plate around screw holes, the deformation of the plate, and the relative motions at the fracture site in complex muscle loading or in the presence on non-transverse fractures.

The proposed design is superior to those in which flax fibres were used as the hydrophilic nature of these fibres promotes high moisture absorption rate, which has been shown to significantly degrade the mechanical properties of the bone plate over time [118]. This paper is unique in that it is the first to consider the optimization of a composite bone plate based on fracture healing requirements. It is also the first study to evaluate the susceptibility to failure of composite bone plates in Vancouver B1 fractures. The proposed composite plate can potentially be used in other long bones such as tibial shaft fractures since the tibia is subjected to a similar load level to the 
femur. However, separate simulations are required to investigate whether the plate is strong enough in the vicinity of the screw holes in the tibia.

\subsubsection{Potential limitations}

The present chapter has certain limitations. Firstly, the derivation of closed-form expressions for structural rigidities of the laminate were based on the classical laminate theory which assumed the plane-stress conditions for the laminate. For instance, the out-of-plane normal and shear stress were considered small compared with in-plane stresses. While these assumptions may be less accurate for thick laminates or those subjected to certain loads, the finite element models did not have such simplifying assumptions, and in fact, both out-of-plane response of the laminate and the interactions amongst the plies were considered in the simulations.

The performance of the proposed bone plate was evaluated in Vancouver B1 periprosthetic fractures only. Further investigations are therefore required in other femoral fracture types or other diaphyseal fractures.

Moreover, there were some limitations associated with the FE model. Linear isotropic material properties were assumed for the bone, while nonlinear, anisotropic, and viscoelastic properties might be more characteristic of the mechanical behaviour of real bones. However, previous FE comparisons of synthetic femurs with human cadaveric femurs suggest that linear material behaviour is a reasonable approximation for real femurs [119, 120]. Furthermore, different callus types (i.e. central, adjacent, and peripheral) were not modeled separately in this study, and their properties were averaged at 4 to 6 weeks after surgery. The present model did not consider the relative motions between the bone-bone cement, bone cement-hip implant and screws-bone constructs as it assumed bonded contacts between the interfaces. However, these assumptions simulate perfect interdigitation of the cortical bone with the threads of the screws, complete adhesion of the bone cement to the hip stem, and full bony ingrowth around the cement mantle and have been used in a number of biomechanical studies [91, 104]. Additionally, the model considered 5 major muscles and the joint reaction force at $45 \%$ of the gait cycle, while other stages of the gait cycle were not included. However, this should not affect the conclusion as this instance of the gait cycle has been shown previously to produce maximum loading of the femur $[112,121]$. 
Due to the repetitive nature of physiological loads, the fatigue behaviour of the proposed design should be evaluated, which could be the focus of future studies. Finally, the in-vivo performance of the proposed composite plate must be evaluated using animal studies and ultimately clinical trials before it can be used as an alternative to conventional metallic plates.

\subsection{Summary}

In this chapter, laminate theory and the finite element method were employed for optimization of a composite bone plate. A hybrid composite made of carbon fibre/epoxy with a flax/epoxy core, which was introduced previously, was optimized by varying the laminate stacking sequence and the contribution of each material, in order to minimize the axial rigidity and maximize the torsional rigidity for a given range of bending rigidity. The initial $14 \times 44^{14}$ possible configurations were reduced to 13 after applying various design criteria. A comprehensive finite element model, validated against a previous experimental study, was used to evaluate the mechanical performance of each composite configuration in terms of its fracture stability, load sharing, and strength in transverse and oblique Vancouver B1 fracture configurations at immediately post-operative, postoperative, and healed bone stages. It was found that a carbon fibre/epoxy plate with an axial rigidity of 4.6 MN, and bending and torsional rigidity of 13 and $14 \mathrm{~N} . \mathrm{m}^{2}$ respectively, showed an overall superiority compared with other laminate configurations. It increased the compressive force at the fracture site up to $14 \%$ when compared to a conventional metallic plate, and maintained fracture stability by ensuring the fracture fragments' relative motions were comparable to those found during metallic plate fixation. The healed stage results revealed that implantation of the titanium plate caused a $40.3 \%$ reduction in bone stiffness, while the composite plate lowered the rigidity by $32.9 \%$ as compared to the intact femur. This study proposed a number of guidelines for the design of composite bone plates. The findings suggest that a composite bone plate could be customized to allow for moderate compressive force on the fracture ends, while remaining relatively rigid in bending and torsion and strong enough to withstand external loads when a fracture gap is present. The results indicate that the proposed composite bone plate could be a potential candidate for bone fracture plate applications. This is the first study to consider the optimization of a composite bone fracture plate using the selective stress shielding approach. This study suggests a number of guidelines when designing composite bone plates. The proposed composite design is superior to those previously developed in that it takes advantage of the tailorability of composite structures to 
achieve desired properties in the required directions, with the aim of developing a flexible yet strong fracture fixation. Beneficial and detrimental mechanical factors that are directly or indirectly involved in fracture healing were employed to evaluate the composite plate's performance in both transverse and oblique fractures. The CF/epoxy composite bone plate increased the compression at fracture ends while minimally affecting the axial and torsional stability of the fracture. The susceptibility of the plate to failure was also assessed using the Tsaihill failure criterion. Strain patterns that were more similar to the intact femur were found with use of the composite bone plate as opposed to the Ti plate following healing which can result in less peri-implant bone loss over time as a result of bone remodelling. The findings also revealed that using a flax/epoxy core for the composite bone plate regardless of its contribution and stacking sequence sacrifices the torsional rigidity and strength of the bone plate and is not superior to a pure carbon/epoxy plate for femoral shaft fracture fixation. The proposed methodology can be used to design composite bone plates for treating fractures of long bones other than the femur, such as the tibia and humerus. However, the required rigidity and strength for the bone plate might be different for weight-bearing bones as opposed to non-weight-bearing ones, and the thus actual loading on the bone should be considered in the design process. The results of this study suggest that the proposed composite bone plate could be a potential candidate for bone plate applications in the femur. 


\section{STRUCTURAL RIGIDITIES OF A COMPOSITE TUBE}

\subsection{Introduction}

To start the optimization process for a composite IM nail (i.e. to minimize the axial rigidity while maximizing bending and torsional rigidities), it is necessary to obtain closed-form expressions for axial, bending, and torsional rigidities in a composite tube. In this chapter, a combination of the classical laminate theory and beam theory was used for derivation of the rigidity formulas.

\subsection{Composite beam governing equations}

In a circular hollow orthotropic beam subjected to an axial force, bending and twist moments (Figure 4-1), the forces-strain relationships are:

$$
\left\{\begin{array}{c}
\bar{N} \\
\bar{M}_{y} \\
\bar{M}_{z} \\
\bar{T}
\end{array}\right\}=\left[\begin{array}{cccc}
E A & 0 & 0 & 0 \\
0 & E I_{y y} & E I_{y z} & 0 \\
0 & E I_{y z} & E I_{z z} & 0 \\
0 & 0 & 0 & G J
\end{array}\right]\left\{\begin{array}{c}
\varepsilon_{x} \\
1 \\
\rho_{y} \\
1 \\
\rho_{z} \\
\xi
\end{array}\right\}
$$

where $\varepsilon_{x}{ }^{0}$ is the axial strain, $\frac{1}{\rho_{y}}$ and $\frac{1}{\rho_{z}}$ are curvatures about $y$ and $z$ axes respectively, and $\xi$ is the rate of twist about the $x$ axis. In order to relate the external forces and moments on the beam to the beam wall, a local coordinate system $(s-n-x)$ is defined (Figure 4-1) with its origin at the laminate mid-surface and its $x$-axis parallel to the $\mathrm{x}$-axis of the beam and its $s$-axis tangent to the beam circular cross section. Thus, $n$-axis is always normal to the beam wall. Based on BernoulliNavier hypothesis [101], the axial strain varies linearly with the curvature of the beam. That said, the axial strain on an arbitrary point across the beam cross section can be written as:

$$
\varepsilon_{x}=\varepsilon_{x}^{0}+z \frac{1}{\rho_{y}}+y \frac{1}{\rho_{z}}
$$




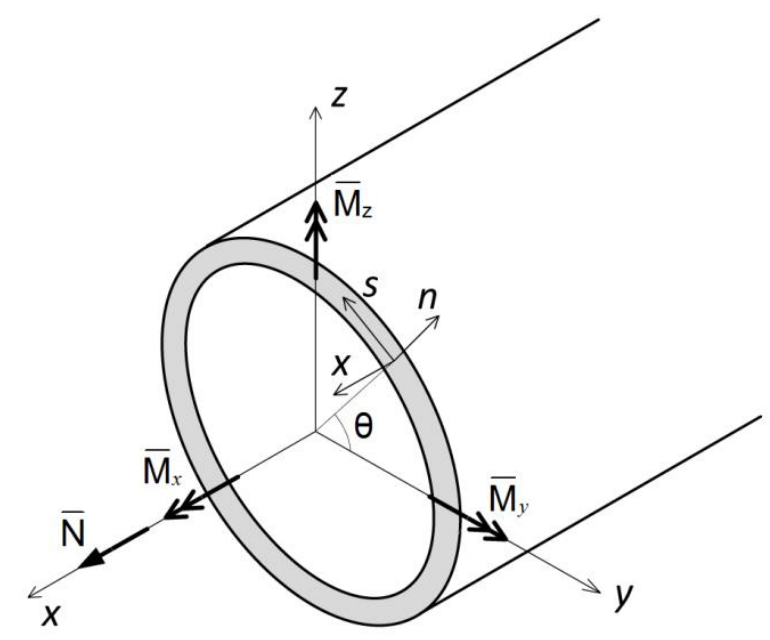

Figure 4-1. The definition of the local coordinate system on the wall

Also, the wall curvature (in $x$ - $n$ plane) can be obtained as a function of beam curvature:

$$
\kappa_{x}=\frac{1}{\rho_{z}} \cos \theta-\frac{1}{\rho_{y}} \sin \theta
$$

where $\theta$ is the angle between the $n$ - and $y$-coordinate axes (Figure 4-1). With the preceding definition of $s-n-x$ coordinate system, one can obtain expressions for the in-plane forces and moments in the laminate as follows:

$$
\left\{\begin{array}{l}
N_{x} \\
N_{s} \\
N_{x s} \\
M_{x} \\
M_{s} \\
M_{x s}
\end{array}\right\}=\left[\begin{array}{llllll}
A_{11} & A_{12} & A_{16} & B_{11} & B_{12} & B_{16} \\
A_{12} & A_{22} & A_{26} & B_{12} & B_{22} & B_{26} \\
A_{16} & A_{26} & A_{66} & B_{16} & B_{26} & B_{66} \\
B_{11} & B_{12} & B_{16} & D_{11} & D_{12} & D_{16} \\
B_{12} & B_{22} & B_{26} & D_{12} & D_{22} & D_{26} \\
B_{16} & B_{26} & B_{66} & D_{16} & D_{26} & D_{66}
\end{array}\right]\left\{\begin{array}{c}
\varepsilon_{x} \\
\varepsilon_{s} \\
\gamma_{x s} \\
\kappa_{x} \\
\kappa_{s} \\
\kappa_{x s}
\end{array}\right\}
$$

in which $A_{i j}, B_{i j}$, and $D_{i j}$ are extensional, coupling, and bending rigidity matrices respectively. Solving for strain components, and considering an unsymmetrical orthotropic laminate yield: 


$$
\left\{\begin{array}{c}
\varepsilon_{x} \\
\varepsilon_{s} \\
\gamma_{x s} \\
\kappa_{x} \\
\kappa_{s} \\
\kappa_{x s}
\end{array}\right\}=\left[\begin{array}{cccccc}
\alpha_{11} & \alpha_{12} & 0 & \beta_{11} & \beta_{12} & 0 \\
\alpha_{12} & \alpha_{22} & 0 & \beta_{12} & \beta_{22} & 0 \\
0 & 0 & \alpha_{66} & 0 & 0 & \beta_{66} \\
\beta_{11} & \beta_{12} & 0 & \delta_{11} & \delta_{12} & 0 \\
\beta_{12} & \beta_{22} & 0 & \delta_{12} & \delta_{22} & 0 \\
0 & 0 & \beta_{66} & 0 & 0 & \delta_{66}
\end{array}\right]\left\{\begin{array}{c}
N_{x} \\
N_{s} \\
N_{x s} \\
M_{x} \\
M_{s} \\
M_{x s}
\end{array}\right\}
$$

where which $\alpha_{i j}, \beta_{i j}$, and $\delta_{i j}$ are elements of $[A]^{-1},[B]^{-1}$, and $[D]^{-1}$ respectively.

Resultant forces and moments applied on the beam can be obtained in terms of in-plane forces and moments as follows:

$$
\begin{aligned}
& \bar{N}=\int_{0}^{2 \pi} N_{x} R d \theta \\
& \bar{M}_{y}=-\int_{0}^{2 \pi} M_{x} R \sin \theta d \theta+\int_{0}^{2 \pi} N_{x} R^{2} \sin \theta d \theta \\
& \bar{M}_{z}=\int_{0}^{2 \pi} M_{x} R \cos \theta d \theta-\int_{0}^{2 \pi} N_{x} R^{2} \cos \theta d \theta \\
& \bar{T}=2 \int_{0}^{2 \pi} M_{x s} R d \theta
\end{aligned}
$$

\subsection{Bending rigidity of a composite tube}

To obtain the bending rigidity, it is assumed that no axial force and twist moment are applied on the beam and that the beam is only subjected to $\bar{M}_{y}$. As a result, (4.2) becomes:

$$
\varepsilon_{x}=z \frac{1}{\rho_{y}}+y \frac{1}{\rho_{z}}
$$

To simplify the formulation, the laminate rigidity elements are evaluated at the laminate neutral surface, in which a bending moment $\left(M_{x}\right)$ does not result in axial strain $\left(\varepsilon_{x}\right)$ in the laminate. Using this definition, the deviation of the laminate neutral surface and the mid-surface is obtained as [101]: 


$$
d=-\frac{\beta_{11}}{\delta_{11}}
$$

Transferring $\alpha_{i j}, \beta_{i j}$, and $\delta_{i j}$ to the neutral surface yields [101]:

$$
\begin{aligned}
& \alpha_{i j}^{\prime}=\alpha_{i j}+d\left(\beta_{i j}+\beta_{i j}\right)+d^{2} \delta_{i j} \\
& \beta_{i j}^{\prime}=\beta_{i j}+d \delta_{i j} \\
& \delta_{i j}^{\prime}=\delta_{i j}
\end{aligned}
$$

As a result of this transformation:

$$
\beta_{11}^{\prime}=0
$$

If the dimensions of the beam cross section are relatively small compared to the beam's length, and the beam layup is orthotropic, the following in-plane forces and moments are infinitesimally small and can be assumed zero [101];

$$
N_{s}=N_{x s}=M_{s}=M_{x s}=0
$$

Also, in the case that the composite beam is manufactured by wrapping or advanced fiber placement (AFP) which is usually the case for composite circular tubes, the layup configurations is referred to as circumferential uniform stiffness (CUS). In such structure, a bending moment in the $y$-direction does not cause a curvature in the $z$-direction [122]. This uncoupling results in:

$$
\frac{1}{\rho_{z}}=0
$$

Combining (4.10), (4.15), (4.16), (4.17), (4.5), and (4.7) yields:

$$
\bar{M}_{y}=\int_{0}^{2 \pi} \frac{1}{\delta_{11}^{\prime}} \sin ^{2} \theta d \theta \frac{1}{\rho_{y}}+\int_{0}^{2 \pi} \frac{1}{\alpha_{11}^{\prime}} R^{\prime 3} \sin ^{2} \theta d \theta \frac{1}{\rho_{y}}
$$

and the coefficient of $\frac{1}{\rho_{y}}$ in the above equation gives the bending rigidity of the orthotropic composite beam with circular cross section. 


$$
E I=\pi\left(\frac{R^{\prime}}{\delta_{11}^{\prime}}+\frac{R^{\prime 3}}{\alpha_{11}^{\prime}}\right)
$$

In symmetric layup, the neutral surface coincides with the laminate mid-surface and (4.19) can be simplified to:

$$
E I=\pi\left(\frac{R}{\delta_{11}}+\frac{R^{3}}{\alpha_{11}}\right)
$$

\subsection{Axial rigidity of a composite tube}

To obtain the axial stiffness, it is assumed that the beam is only subjected to an axial force and there is no moments applied on it. In a circular cross section beam with CUS layup, an axial force does not cause any bending [122, 123].This uncoupling reduces (4.2) and (4.3) to:

$$
\begin{aligned}
& \varepsilon_{x}=\varepsilon_{x}{ }^{0} \\
& \kappa_{x}=0
\end{aligned}
$$

Also, in such beam the curvature does not change along the circumference [101]

$$
\kappa_{s}=0
$$

Thus, and (4.5) yields:

$$
\begin{aligned}
& \varepsilon_{x}=\alpha_{11} N_{x}+\beta_{11} M_{x}+\beta_{12} M_{s} \\
& \kappa_{x}=\beta_{11} N_{x}+\delta_{11} M_{x}+\delta_{12} M_{s} \\
& \kappa_{s}=\beta_{12} N_{x}+\delta_{12} M_{x}+\delta_{22} M_{s}
\end{aligned}
$$

Combining (4.23), (4.24), and solving for $N_{x}$ and $M_{x}$ :

$$
N_{x}=\frac{1}{D^{*}}\left(\delta_{11}-\frac{\delta_{12}^{2}}{\delta_{22}}\right) \varepsilon_{x}-\frac{1}{D^{*}}\left(\beta_{11}-\frac{\beta_{12} \delta_{12}}{\delta_{22}}\right) \kappa_{x}
$$




$$
M_{x}=\frac{1}{D^{*}}\left(\frac{\beta_{12} \delta_{12}}{\delta_{22}}-\beta_{11}\right) \varepsilon_{x}-\frac{1}{D^{*}}\left(\alpha_{11}-\frac{\beta_{12}^{2}}{\delta_{22}}\right) \kappa_{x}
$$

where

$$
D^{*}=\left(\delta_{11}-\frac{\delta_{12}^{2}}{\delta_{22}}\right)\left(\alpha_{11}-\frac{\beta_{12}^{2}}{\delta_{22}}\right)-\left(\beta_{11}-\frac{\beta_{12} \delta_{12}}{\delta_{22}}\right)^{2}
$$

Combining (4.26) with (4.6), the coefficient of axial strain yields the axial rigidity of composite tube with circular cross section and an orthotropic layup as

$$
E A=2 \pi R \frac{1}{D^{*}}\left(\delta_{11}-\frac{\delta_{12}^{2}}{\delta_{22}}\right)
$$

In case of a symmetric layup, the above equation reduces to:

$$
E A=\frac{2 \pi R}{\alpha_{11}}
$$

\subsection{Torsional rigidity of a composite tube}

To calculate the torsional stiffness, it is assumed that the beam is subjected to a twist moment only. In a composite beam with CUS configurations, this twist moment doe not cause any bending [122]. In fact, it induces shear flow $q$ in the wall thickness defined as:

$$
q=\int_{0}^{t} \tau_{x s} d n=N_{x s}
$$

where $t$ is the wall thickness. The external twist moment can be related to the beam shear follow as:

$$
\bar{T}=\oint q R d s=2 q A
$$

in which $A$ is the swept area of the wall mid-surface. (4.5) yields:

$$
\gamma_{x s}=\alpha_{66} N_{x}+\beta_{66} M_{x s}
$$


This equation can be simplified by selecting a "twisting moment neutral" surface (TNS) at which $\beta_{66}$ is zero. Such surface can be found using equation (4.13). The distance between the wall midsurface and the TNS would be:

$$
e=-\frac{\beta_{66}}{\delta_{66}}
$$

Obtaining $\gamma_{x s}$ on the TNS yields:

$$
\gamma_{x s}=\alpha_{66}^{\prime \prime} N_{x s}
$$

where $\alpha_{66}^{\prime \prime}$ is the transferred $\alpha_{66}$ to the TNS. For a circular composite tube with uniform walls thickness, the warping is zero. This results in the rate of twist to be:

$$
\xi=\oint_{S^{\prime \prime}} \frac{\gamma_{x s}}{2 A^{\prime \prime}} d s
$$

in which $A^{\prime \prime}$ is the swept area of the TNS. Combining (4.37), (4.36), and (4.33), and integrating over the TNS yields:

$$
\xi=\frac{2 \pi R^{\prime \prime 3}}{\alpha_{66}^{\prime \prime}} \bar{T}
$$

From which the torsional rigidity of a composite tube with circular cross section is obtained as:

$$
G J=\frac{2 \pi R^{\prime \prime 3}}{\alpha_{66}^{\prime \prime}}
$$

When considering a symmetric layup for the composite tube, TNS coincides with the laminate mid-surface and the above equation reduces to:

$$
G J=\frac{2 \pi R^{3}}{\alpha_{66}}
$$




\subsection{Validation of the rigidity formulas}

In order to verify the validity of assumptions in derivation of the rigidity formulas, a 3-dimensional (3D) finite element (FE) model was employed. This model took into account the out-of-plane response of the laminate and the interactions amongst the plies.

The computer-aided design (CAD) model of a cylinder with an outer diameter of $14 \mathrm{~mm}$ (similar to that of a conventional Ti-alloy IM nail) was created in ANSYS Workbench software (ANSYS Inc., Canonsburg, PA, USA). ANSYS Composite PrepPost module was employed to assign the stacking sequence of the laminates. Each layer was meshed with solid (SOLID 187) elements. Ten different lay-ups with 16 plies of CF/epoxy were considered, as tabulated in Table 4-1, and the structural rigidities were obtained in the FE model as follows:

To obtain the axial rigidity, an axial force of $1000 \mathrm{~N}$ was applied on the beam cross-section and the axial displacement of the beam was obtained from the FE model. Using Eq. (4.41), the axial rigidity of the beam was calculated.

$$
E A=\frac{F L}{D_{\max }}
$$

In this equation, $F$ is the applied axial force, $L$ is the length of the beam and $D_{\max }$ is the maximum axial displacement of the beam which occurs where the axial force is applied.

A standard four-point bending test was simulated to obtain the bending rigidity of the composite beam. In this test, two normal equidistant forces (with a total magnitude of $1000 \mathrm{~N}$ ) were applied on a beam which was simply supported as depicted in Figure 4-2. The maximum displacement of the beam, occurred and the middle, was measured for each laminate configurations and the bending rigidity of the beam was calculated using Eq. (4.42)

$$
E I=\frac{F L^{3}}{64 D_{\max }}
$$

where $F$ is the total force, $L$ is the length of the beam (between the two supports), and $D_{\max }$ is the maximum displacement of the beam.

A standard torsion test was simulated to obtain the torsional rigidity of the beam. In this test, a twisting moment of $50 \mathrm{~N} . \mathrm{m}^{2}$ was applied to one end of the beam, while the other end was 
prevented from twisting. The angle of twist as a result of the applied moment was measured in the FE model and the torsional rigidity of the beam was obtained using Eq. (4.43)

$$
G J=\frac{T L}{\theta_{\max }}
$$

where $T$ is the applied twisting moment, $L$ is the length of the beam and $\theta_{\max }$ is the maximum twist angle.

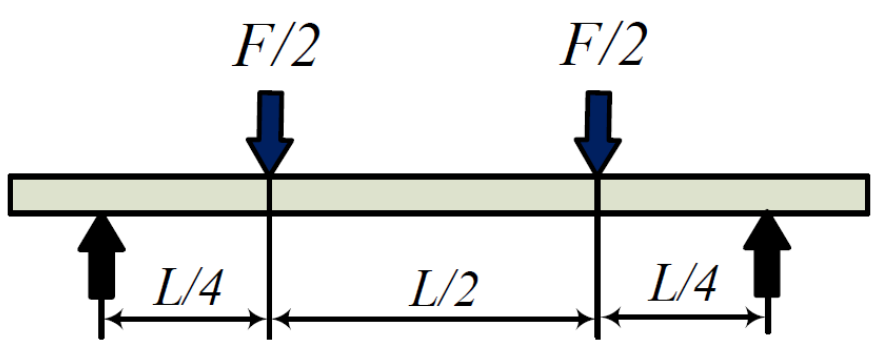

Figure 4-2. The standard testing setup for the four-point bending tests used in the simulations

The rigidity values obtained from the closed-form expressions were compared to those obtained through 3D FE models in Figure 4-3 and 4-5. The slopes of the graphs (1.015, 1.1385, and 0.9486) and correlation coefficients $(0.998,0.989$, and 0.989$)$ were both close to the ideal value of 1 , indicating excellent agreement between the predicted values of rigidity by the close-form expressions and 3D FE simulations. 


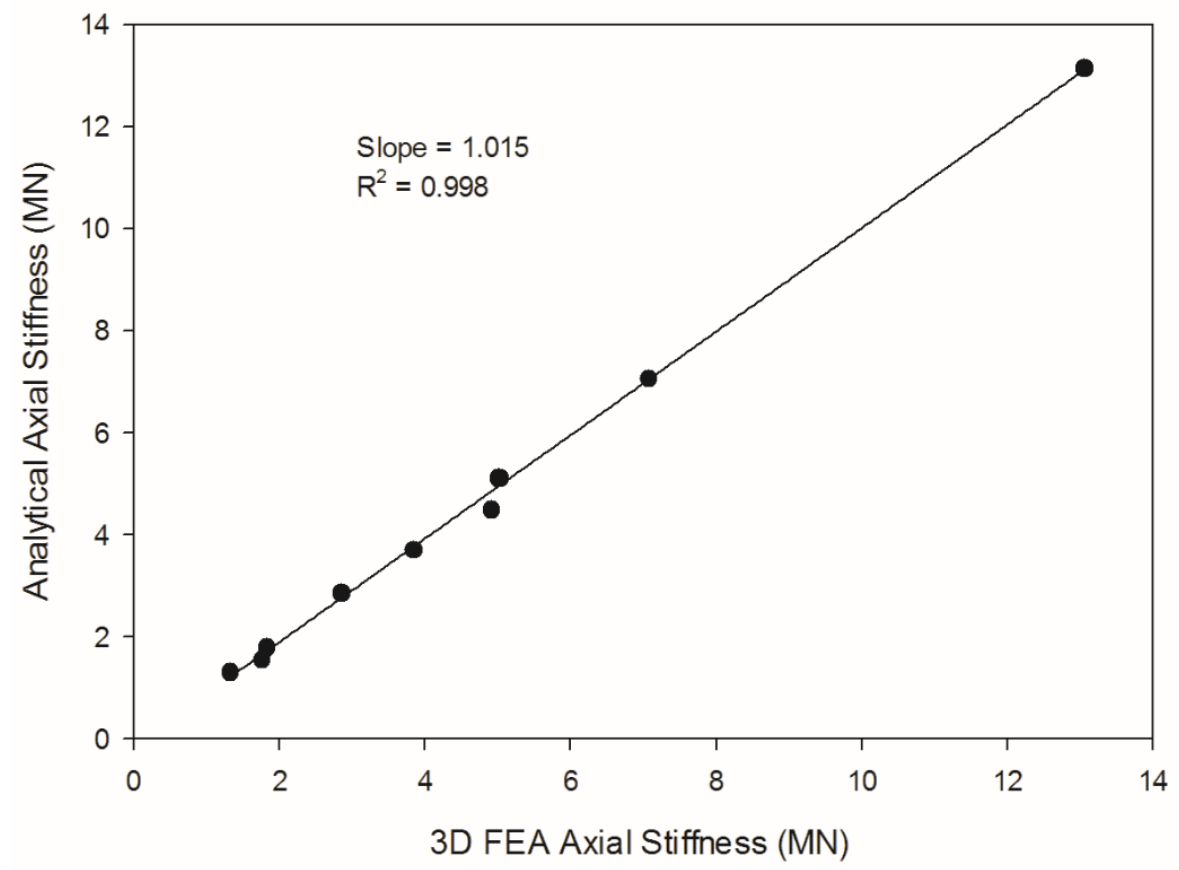

Figure 4-3. Axial rigidity values obtained from closed-form expression versus those obtained from 3D FEA

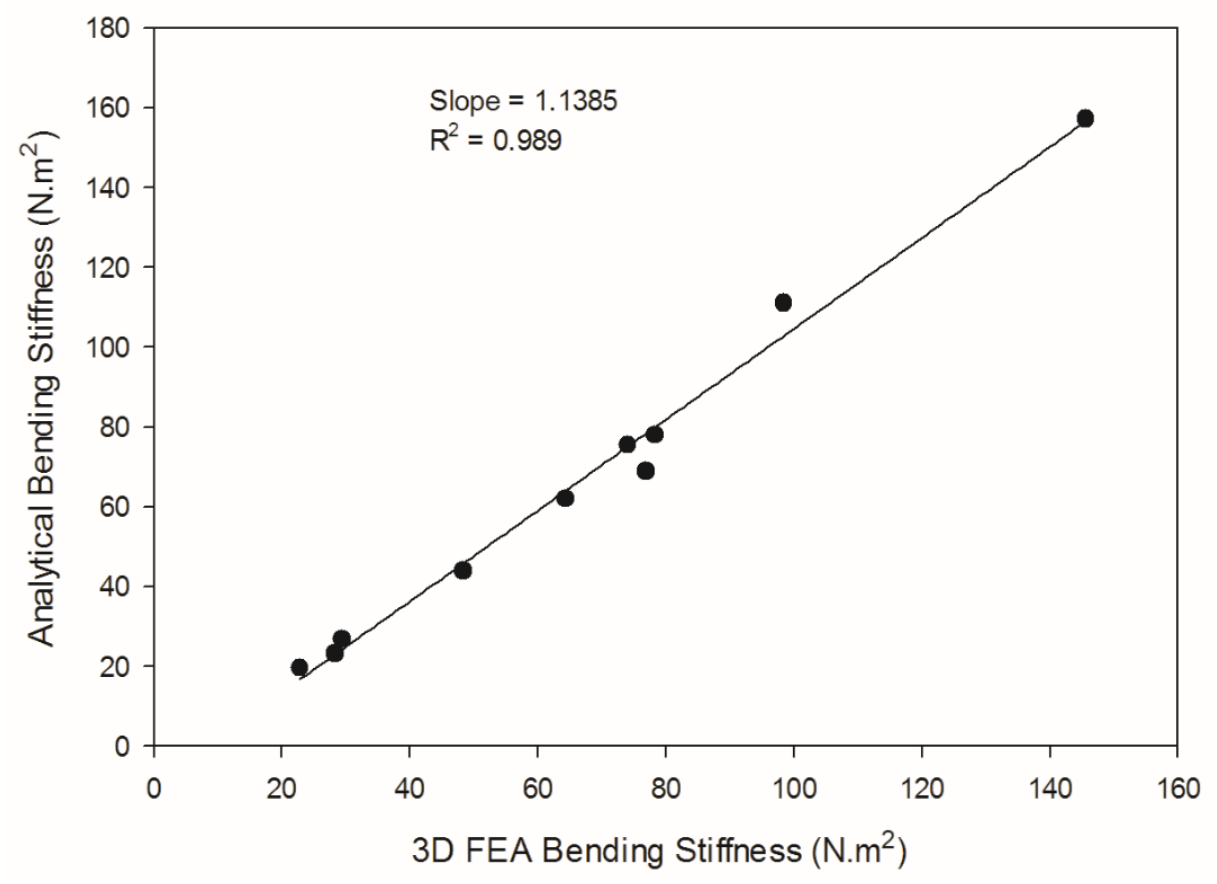

Figure 4-4. Bending rigidity values obtained from 3D FEA versus those obtained from closedform expression 


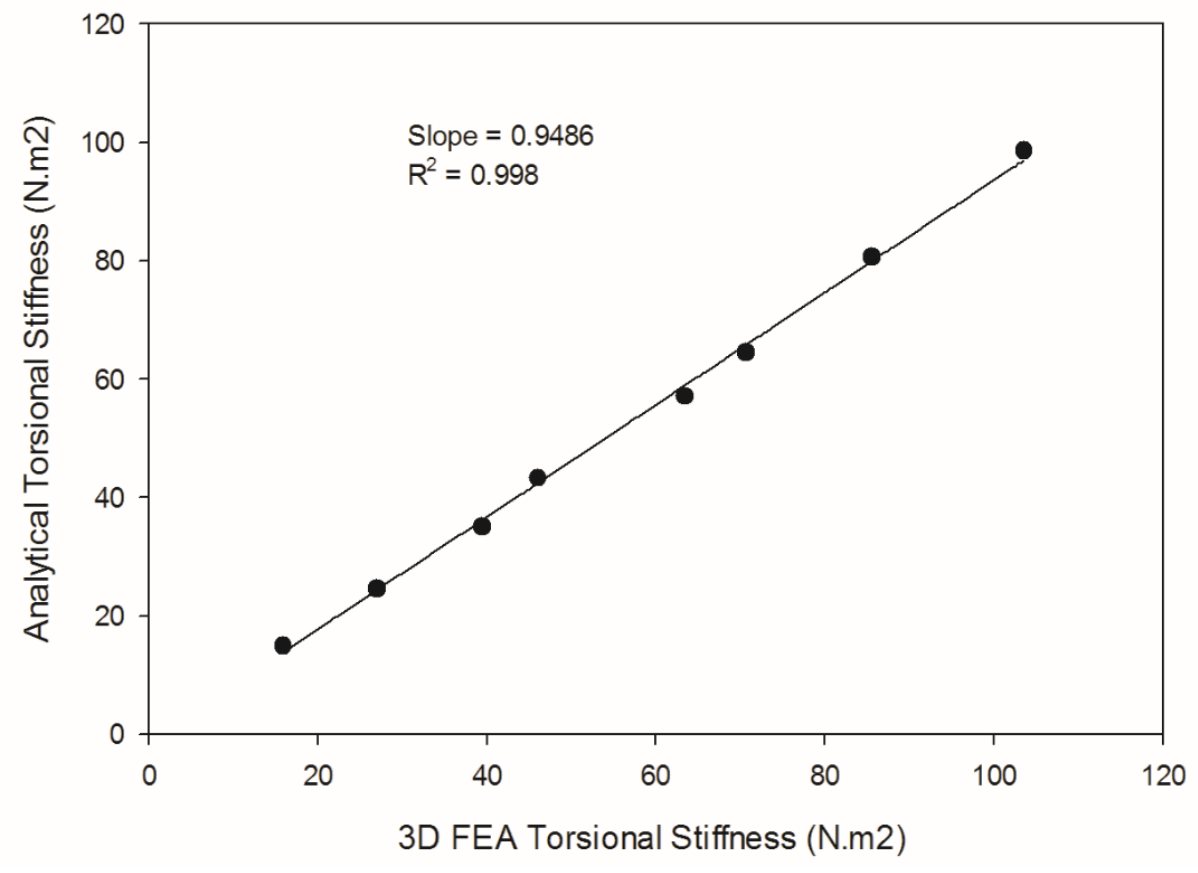

Figure 4-5.Torsional rigidity values obtained from 3D FEA versus those obtained from closedform expression

\subsection{Summary}

Closed-form expressions for axial, bending, and torsional rigidities of a composite tube were derived by combining the classical laminate theory and the beam theory. The obtained expressions were validated against numerical results obtained from finite element analysis. Good correlations were found between the theoretical and numerical values. The formulas can be used in the optimization process of the composite IM nail structure. 
Table 4-1. Different lay-up configurations used for validation of the rigidity formulas

\begin{tabular}{l|ll} 
Lay-up & Stacking Sequence & Laminate Type \\
\hline C1 & {$[45]_{16}$} & Unidirectional, Symmetric \\
\hline C2 & {$\left[45_{8} /-45_{8}\right]$} & Angle-Ply, Balanced \\
\hline C3 & {$[ \pm 45]_{8}$} & Angle-Ply, Balanced \\
\hline C4 & {$\left[0_{2} / 45_{4} / 90_{4} /-45_{4} / 0_{2}\right]$} & Balanced \\
\hline C5 & {$\left[0 / 45_{6} / 90\right]_{S}$} & Symmetric \\
\hline C6 & {$[0]_{16}$} & $\begin{array}{l}\text { Unidirectional, Cross-Ply, } \\
\text { Symmetric }\end{array}$ \\
\hline C7 & {$[0 / 90]_{8}$} & Cross-Ply, Balanced \\
\hline C8 & {$\left[0 / 45_{2} / 90_{2} /-45_{2}\right]_{S}$} & $\begin{array}{l}\text { Quasi-isotropic, } \\
\text { Symmetric }\end{array}$ \\
\hline C9 & {$\left[-45 / 45 / 0_{2} / 45 /-45 / 90_{2} / 45 /-45_{2} / 45 / 0_{2} / 90_{2}\right]$} & Balanced \\
\hline C10 & {$\left[0 /-45 / 45_{2} /-45 / 0 /-45 / 45_{2} /-45 / 45 /-45_{2} / 45 / 90_{2}\right]$} & Balanced \\
\hline
\end{tabular}




\section{STRUCTURAL COUPLINGS IN A COMPOSITE TUBE}

\subsection{Introduction}

Despite the advantages of composite IM nails, the couplings in the tube structure which can arise with particular stacking sequences, can cause undesirable deformations and interfere with its performance as a fracture fixation device. For instance, an extension-bending coupling can bend the IM nail even when the structure experiences pure compressive load, resulting in uneven compression at the fracture site, which in turn can be detrimental to fracture healing.

Hollow composite beams can be manufactured with circumferentially asymmetric stiffness (CAS) or circumferentially uniform stiffness (CUS) configurations. In the former configuration, extension-bending and bending-twist couplings can be produced even with a symmetric beam cross-section [124]. As a result, an applied axial load can produce coupled axial deflection and bending of the beam, and a bending moment can twist the beam structure. In the latter configuration, there is no extension-bending or bending-twist coupling present. However, a composite tube with CUS configuration can still produce extension-twist coupling [125]. In a composite IM nail, such coupling can twist the fracture ends when the fracture site is in compression and can increase the relative shear movement of both ends. The situation can occur in daily physiologic activities and may delay the healing process.

Circular composite tubes are commonly manufactured by automated fiber placement (AFP) or filament winding techniques which lead to a CUS configuration. The ratio of the wall thickness to the laminate mid-plane diameter in a composite IM nail is usually greater than 0.1 [126], and thus the thin-wall theory assumptions for the beam structure may not be valid.

There are several studies in the literature which investigated the structural behavior of thin-walled composite tubes $[101,123,127,128]$, but there are few studies on the structural response of thickwalled circular composite tubes [122, 129]. In particular, there seem to be no criteria (e.g. a factor) that can quantitatively express the extension-twist coupling in thick-walled circular composite tubes which have numerous practical applications.

This chapter aims at introducing a quantitative criteria (i.e., a factor) to detect and measure extension-twist coupling in circular composite tubes with CUS configuration. Such factor could then be employed in the design process of composite IM nails to uncouple extension and twist in 
the structure. In the first part of this chapter, the derivation of constitutive equations for the beam was performed considering out-of-plane displacements of the beam cross-section due to bending and shear. The assumptions took into account the transverse shear effects of the wall which also makes the formulation valid for thick-walled composite tubes. A closed-form solution for the extension-twist coupling factor was derived by obtaining resultant forces and moments over the beam cross-section and then by finding force-displacement relations. In the second part of the chapter, the extension-twist coupling factor was obtained for ten composite tubes with different stacking sequences, and a 3D finite element model was employed to capture such coupling.

\subsection{Analysis}

\subsubsection{Basic assumptions}

The system under study was a hollow composite beam, with a thickness of $h$, and a length of $L$ made of homogenous elastic orthotropic material. The beam had a circular cross-section and the wall consisted of a composite laminate. The radius of the mid-plane of the wall was $R$ and it was assumed that $R<<$ making the 1D beam theory assumptions valid. Also there was no restriction on the wall thickness of the beam. The beam was subjected to an axial force $(N)$ and a twisting moment (twisting moment) $(T)$. Analysis was started by defining the displacement field of a general point on the beam cross-section.

\subsubsection{Displacement field}

The circular beam geometry is normally described by two coordinate systems, namely $s-z-n$ and $x-y-z$. The former was a local curvilinear coordinate system located on the mid-plane of the wall thickness whose $s$-axis was tangent to the mid-plane of the wall at any point. The normal coordinate $n$ was measured along the normal to the mid-plane towards the outer side (Figure 5-1). The latter system was a Cartesian coordinate system placed at the centre of the beam cross-section [127]. As depicted in Figure 5-1, the z-axes of both coordinate systems coincided. The 3D displacement field of a general point on the cross-section was expressed with respect to these coordinate systems considering the following assumptions [123, 130-132]:

- The out-of-plane displacement of the beam cross-section as a result of bending and shear was described by a cubic function of the cross-sectional coordinates $x$ or $y$. This indicates 
that the transverse shear effects of the beam were considered and that the transverse shear strains vary parabolically across the thickness.

- The beam cross-section did not deform in its own plane.

- All deformations were small and within linear elasticity.

Using the kinematic assumptions, the beam displacement field was assumed to be $[122,130]$ :

$$
\begin{aligned}
& w(z, \alpha, n)=w_{0}(z)-r_{x}\left[\theta_{x}(z)+\frac{4(R+n)^{3} \cos ^{3} \alpha}{3 R_{o}^{3}}\left(u_{0}^{\prime}(z)-\theta_{x}(z)\right)\right] \\
& -r_{y}\left[\theta_{y}(z)+\frac{4(R+n)^{3} \sin ^{3} \alpha}{3 R_{o}^{3}}\left(v_{0}^{\prime}(z)-\theta_{y}(z)\right)\right]-\psi(\alpha, n) \phi^{\prime}(z)
\end{aligned}
$$

$u_{s}(z, \alpha, n)=-u_{0}(z) \sin \alpha+v_{0}(z) \cos \alpha+(R+n) \phi(z)$

$u_{n}(z, \alpha, n)=u_{0}(z) \cos \alpha+v_{0}(z) \sin \alpha$

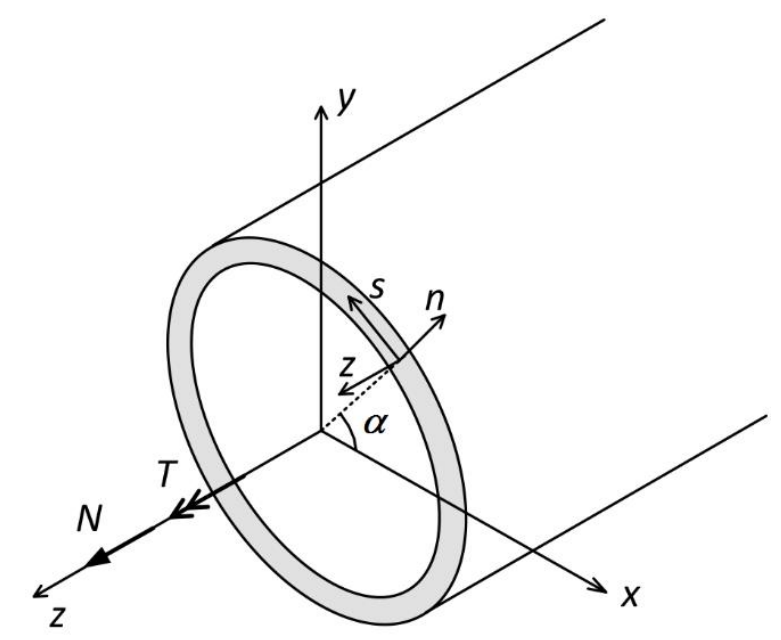

Figure 5-1. The definition of the local coordinate system on the wall and the global coordinate system at the centre of the composite beam cross-section 
The functions $u_{0}(z), v_{0}(z)$, and $w_{0}(z)$ represent the rigid body translations along the $x, y$, and $z$ axes, while the rigid body rotation about $x$ and $y$ axes are denoted by $\theta_{x}(z), \theta_{y}(z)$. The warping function is denoted by $\psi(\alpha, n)$, and $u_{s}, u_{n}$, and $w$ are curvilinear tangential, normal, and axial displacements respectively, and $\phi(z)$ is the angle of twist. The prime denotes derivatives with respect to the span of the beam (z-coordinate). In Eqs. (5.1-3), the angle $\alpha$ was used to represent the circumferential coordinate $s$ since the radius of the mid-plane of the wall $(R)$ was constant in a circular tube. In these equations, $R_{o}$ is the outer radius of the beam. The functions $r_{x}$ and $r_{y}$ denote the $x$ and $y$ components of the position with respect to the global coordinate system and are defined as:

$$
\begin{aligned}
& r_{x}=(R+n) \cos \alpha \\
& r_{y}=(R+n) \sin \alpha
\end{aligned}
$$

It is worth noting that the term $(R+n) \phi(z)$ in Eq. (5.2) is not considered in thin-walled composite beam theories $[123,127]$.

\subsubsection{Strain field}

The beam strain field can be derived by differentiating the displacement field. Based on the assumption of a cross-section without in-plane deformations as well as the 1D formulation in beam theory, the strain filed was expressed by:

$$
\begin{aligned}
& \varepsilon_{z z}=\frac{\partial w}{\partial z} \\
& \gamma_{z s}=\frac{1}{R} \frac{\partial w}{\partial \alpha}+\frac{\partial u_{s}}{\partial z} \\
& \gamma_{z n}=\frac{\partial w}{\partial \alpha} n+\frac{\partial u_{n}}{\partial z}
\end{aligned}
$$




\subsubsection{Stress-strain relationship}

For a generally orthotropic $i^{\text {th }}$ layer of a laminate, the stresses and the strains are related through the following equation in which $\bar{Q}_{a b}$ are the elements of the transformed stiffness matrix for an orthotropic material [101].

$$
\left[\begin{array}{c}
\sigma_{z z} \\
\sigma_{s s} \\
\sigma_{n n} \\
\tau_{s n} \\
\tau_{z n} \\
\tau_{z s}
\end{array}\right]_{i}\left[\begin{array}{cccccc}
\bar{Q}_{11} & \bar{Q}_{12} & \bar{Q}_{13} & 0 & 0 & \bar{Q}_{16} \\
\bar{Q}_{12} & \bar{Q}_{22} & \bar{Q}_{23} & 0 & 0 & \bar{Q}_{26} \\
\bar{Q}_{13} & \bar{Q}_{23} & \bar{Q}_{33} & 0 & 0 & \bar{Q}_{36} \\
0 & 0 & 0 & \bar{Q}_{44} & \bar{Q}_{45} & 0 \\
0 & 0 & 0 & \bar{Q}_{45} & \bar{Q}_{55} & 0 \\
\bar{Q}_{16} & \bar{Q}_{26} & \bar{Q}_{36} & 0 & 0 & \bar{Q}_{66}
\end{array}\right]_{i}\left[\begin{array}{c}
\varepsilon_{z z} \\
\varepsilon_{s s} \\
\varepsilon_{n n} \\
\varepsilon_{s n} \\
\varepsilon_{z n} \\
\varepsilon_{z s}
\end{array}\right]_{i}
$$

In $1 \mathrm{D}$ beam theory, the off-cross sectional components of stress (i.e. $\sigma_{s s}, \sigma_{n n}$, and $\tau_{s n}$ ) are considered negligibly small compared to the remaining stress components [122]. However, the contribution of off-cross sectional strains to the beam deformation may become significant as the wall thickness increases [133]. This is why the contribution of strain components $\varepsilon_{s s}, \varepsilon_{n n}$, and $\gamma_{s n}$ were taken into account in the current formulation.

Setting $\sigma_{s s}, \sigma_{n n}$, and $\tau_{s n}$ equal to zero in Eq. (5.9), in-plane normal strain $\left(\varepsilon_{s s}\right)$, transverse normal strain $\left(\varepsilon_{n n}\right)$ and transverse shear strain $\left(\gamma_{s n}\right)$ can be calculated in terms of $\varepsilon_{z z}$ and $\gamma_{z s}$ as follows:

$$
\begin{aligned}
& \varepsilon_{s s}=\frac{\bar{Q}_{13} \bar{Q}_{23}-\bar{Q}_{12} \bar{Q}_{33}}{\bar{Q}_{22} \bar{Q}_{33}-\bar{Q}_{23}^{2}} \varepsilon_{z z}+\frac{\bar{Q}_{23} \bar{Q}_{36}-\bar{Q}_{26} \bar{Q}_{33}}{\bar{Q}_{22} \bar{Q}_{33}-\bar{Q}_{23}^{2}} \gamma_{z s} \\
& \varepsilon_{n n}=\frac{\bar{Q}_{23} \bar{Q}_{12}-\bar{Q}_{22} \bar{Q}_{13}}{\bar{Q}_{22} \bar{Q}_{33}-\bar{Q}_{23}^{2}} \varepsilon_{z z}+\frac{\bar{Q}_{26} \bar{Q}_{23}-\bar{Q}_{22} \bar{Q}_{36}}{\bar{Q}_{22} \bar{Q}_{33}-\bar{Q}_{23}^{2}} \gamma_{z s} \\
& \gamma_{s n}=-\frac{\bar{Q}_{45}}{\bar{Q}_{44}}
\end{aligned}
$$

The three remaining stress components for the $i^{\text {th }}$ layer can be written as follows: 


$$
\left[\begin{array}{l}
\sigma_{z z} \\
\sigma_{z s} \\
\sigma_{z n}
\end{array}\right]_{i}=\left[\begin{array}{ccc}
C_{11} & C_{12} & 0 \\
C_{12} & C_{22} & 0 \\
0 & 0 & C_{33}
\end{array}\right]_{i}\left[\begin{array}{l}
\varepsilon_{z z} \\
\varepsilon_{z s} \\
\varepsilon_{z n}
\end{array}\right]_{i}
$$

in which

$$
\begin{aligned}
& C_{11}=\bar{Q}_{11}+\frac{2 \bar{Q}_{12} \bar{Q}_{13} \bar{Q}_{23}-\bar{Q}_{12}^{2} \bar{Q}_{33}-\bar{Q}_{13}^{2} \bar{Q}_{22}}{\bar{Q}_{22} \bar{Q}_{33}-\bar{Q}_{23}^{2}} \\
& C_{12}=\bar{Q}_{16}+\frac{\bar{Q}_{12} \bar{Q}_{23} \bar{Q}_{36}-\bar{Q}_{12} \bar{Q}_{26} \bar{Q}_{33}+\bar{Q}_{13} \bar{Q}_{23} \bar{Q}_{26}-\bar{Q}_{13} \bar{Q}_{22} \bar{Q}_{36}}{\bar{Q}_{22} \bar{Q}_{33}-\bar{Q}_{23}^{2}} \\
& C_{22}=\bar{Q}_{66}+\frac{2 \bar{Q}_{23} \bar{Q}_{26} \bar{Q}_{36}-\bar{Q}_{26}^{2} \bar{Q}_{33}-\bar{Q}_{36}^{2} \bar{Q}_{22}}{\bar{Q}_{22} \bar{Q}_{33}-\bar{Q}_{23}^{2}} \\
& C_{33}=\bar{Q}_{55}-\frac{\bar{Q}_{45}^{2}}{\bar{Q}_{44}}
\end{aligned}
$$

\subsubsection{Axial force and twisting moment equations}

The resultant axial force in the $z$-direction and the twisting moment on the beam could be obtained by integrating related stresses cover the beam cross-section as follows:

$$
\begin{aligned}
& N(z)=\int_{0}^{2 \pi} \int_{-h / 2}^{h / 2} R \sigma_{z z} d n d \alpha \\
& T(z)=\int_{0}^{2 \pi} \int_{-h / 2}^{h / 2} R(R+n) \tau_{s z} d n d \alpha
\end{aligned}
$$

in which $h$ is the wall thickness of the beam. Combining Eqs. (5.1), (5.6), and (5.13) with Eq. (5.18) for the axial force and Eqs. (5.1-2), (5.6-7), and (5.13) with Eqs. (5.19) for the twisting moment result in:

$$
\left[\begin{array}{l}
N(z) \\
T(z)
\end{array}\right]=\left[\begin{array}{ll}
a_{11} & a_{12} \\
a_{21} & a_{22}
\end{array}\right]\left[\begin{array}{l}
w_{0}{ }^{\prime}(z) \\
\phi(z)
\end{array}\right]
$$

where 


$$
\begin{aligned}
& a_{11}=2 \pi R \sum_{i=1}^{k}\left(C_{11}\right)_{i}\left(h_{i}-h_{i-1}\right) \\
& a_{12}=\pi R \sum_{i=1}^{k}\left(C_{12}\right)_{i}\left[\left(R+h_{i}\right)^{2}-\left(R+h_{i-1}\right)^{2}\right]=a_{21} \\
& a_{22}=\frac{2 \pi R}{3} \sum_{i=1}^{k}\left(C_{22}\right)_{i}\left[\left(R+h_{i}\right)^{3}-\left(R+h_{i-1}\right)^{3}\right]
\end{aligned}
$$

The coefficient $a_{12}$ resulted in a coupling between extension and twist. To eliminate such coupling, the following expression must be satisfied:

$$
\lambda=\sum_{i=1}^{k}\left(C_{12}\right)_{i}\left[\left(R+h_{i}\right)^{2}-\left(R+h_{i-1}\right)^{2}\right]=0
$$

The coefficient $\lambda$ is not a function of plies' material properties and only depends on the lay-up stacking sequence provided that the thickness and the material properties of all plies are identical.

\subsection{Numerical results}

\subsubsection{Finite element model}

To examine the coupling between extension and twist in a composite tube, a 3D finite element model was developed which considered the out-of-plane response of the laminate and the interactions amongst the plies. In this regard, ANSYS Workbench 15.0 software (ANSYS Inc., Canonsburg, PA, USA) was utilized to generate the computer-aided design (CAD) models of the cylindrical tubes and ANSYS Composite Prep-Post module was employed to assign the stacking sequence of the laminates using 3D solid elements (Figure 5-2). In this context, each ply was meshed using 8-noded solid (i.e. SOLID185) elements with three degrees of freedom at each node, and was assigned orthotropic material properties taking into account the ply's angle and thickness. 


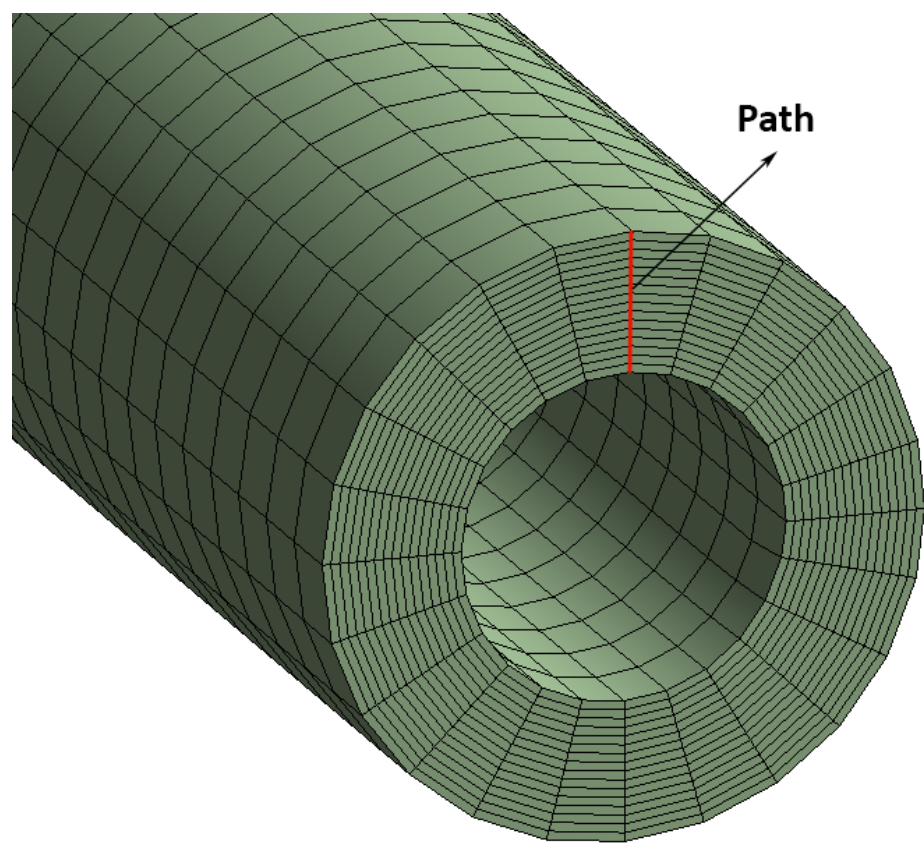

Figure 5-2. Cylindrical composite tube in which each ply is meshed with 8-noded solid elements. The path on which the axial displacement was measured is shown.

The outer diameter of the tubes was selected based on the typical outer diameter of conventional IM nails (i.e. $14 \mathrm{~mm}$ ), and the length of tubes was selected as $250 \mathrm{~mm}$ to make the 1D beam theory assumption valid. This is also because conventional metallic IM nails are made 250-480 $\mathrm{mm}$ long. Ten lay-ups (C1-C10) made of carbon fiber (CF)/epoxy with the same number of plies but different stacking sequences were examined. The elastic material properties of CF/epoxy sheets used in this chapter were tabulated in Table 5-1. The lay-ups with their stacking sequences were included in Table 5-2. A twisting moment of $100 \mathrm{~N} . \mathrm{m}$ was applied on one end and the nodes on the other end were restrained from rotation around the tube axis.

Table 5-1. Linear elastic material properties of prepreg CF/epoxy plies

\begin{tabular}{ccccc}
$E_{X}$ & $E_{Y}$ & $G_{X Y}$ & $v_{X Y}$ & $v_{Y Z}$ \\
$(G P a)$ & $(G P a)$ & $(G P a)$ & & \\
\hline 121 & 8.6 & 4.7 & 0.27 & 0.4
\end{tabular}

*The plies were made of unidirectional (UD) and carbon sheets, with their $X$-axis aligned with the axis of the IM nail. 
To examine the extension-twist coupling in the tubes, the axial displacement of the set of nodes located on a typical line passing the centre of the tube on the surface where the twisting moment was applied (Figure 5-2), was obtained from the FE model. Figure 5-3 depicts the axial nodal displacements for each lay-up. The mean value and standard deviation of the axial displacements were also included in Table 5-2. Using a computer code developed in MATLAB (Mathworks Inc., MA, USA), the coupling factor was calculated for the aforementioned lay-ups and was also included in Table 5-2.

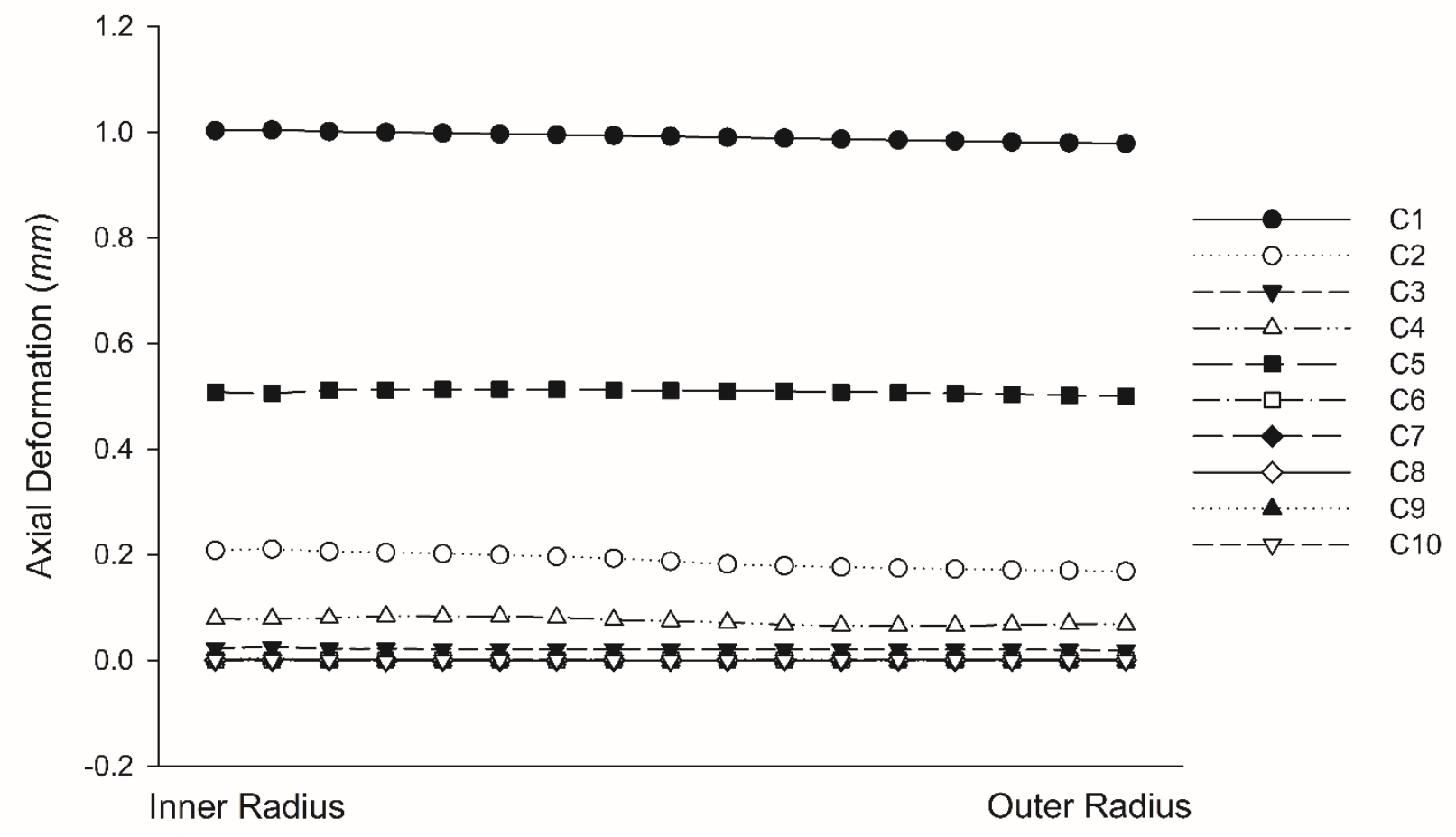

Figure 5-3. Axial deformation of the nodes located on a typical path on the beam wall as a result of applied twisting moment of 100 N.m. 
Table 5-2. Different lay-ups used in the current simulations, their corresponding $a_{12}$ coefficient, and their axial displacements following application of a $100 \mathrm{~N} . \mathrm{m}$ twisting moment.

\begin{tabular}{l|l|l|l|l}
$\begin{array}{l}\text { Lay- } \\
\text { up }\end{array}$ & Stacking Sequence & $\begin{array}{l}\text { Laminate } \\
\text { Type }\end{array}$ & $|\lambda|(\mathbf{N})$ & $\begin{array}{l}\text { Axial Disp. } \\
(\mathbf{m m})\end{array}$ \\
\hline C1 & {$[45]_{16}$} & UD, S & $1.3 \times 10^{6}$ & $0.99 \pm 0.008$ \\
\hline C2 & {$\left[45_{8} /-45_{8}\right]$} & AP, B & $1.4 \times 10^{5}$ & $0.19 \pm 0.01$ \\
\hline C3 & {$[ \pm 45]_{8}$} & AP, B & $1.7 \times 10^{4}$ & $0.02 \pm 0.001$ \\
\hline C4 & {$\left[0_{2} / 45_{4} / 90_{4} /-45_{4} / 0_{2}\right]$} & B & $6.8 \times 10^{4}$ & $0.07 \pm 0.006$ \\
\hline C5 & {$\left[0 / 45_{6} / 90\right]_{S}$} & S & $9.3 \times 10^{5}$ & $0.51 \pm 0.003$ \\
\hline C6 & {$[0]_{16}$} & UD, CP, S & 0 & $(1.1 \pm 2.3) \times 10^{-5}$ \\
\hline C7 & {$[0 / 90]_{8}$} & CP, B & 0 & $(2.0 \pm 2.0) \times 10^{-5}$ \\
\hline C8 & {$\left[0_{2} / 45_{2} / 90_{2} /-45_{2}\right]_{S}$} & QI, S & 0 & $(2.9 \pm 3.8) \times 10^{-4}$ \\
\hline C9 & {$\left[-45 / 45 / 0_{2} / 45 /-45 / 90_{2} / 45 /-45_{2} / 45 / 0_{2} / 90_{2}\right]$} & B & 0 & $(4.8 \pm 9.9) \times 10^{-4}$ \\
\hline C10 & {$\left[0 /-45 / 45_{2} /-45 / 0 /-45 / 45_{2} /-45 / 45 /-45_{2} / 45 / 90_{2}\right]$} & B & 0 & $(1.6 \pm 8) \times 10^{-4}$ \\
\hline
\end{tabular}

* UD (Unidirectional), AP (Angle-ply), S (Symmetric), CP (Cross-ply), B (Balanced), QI

(Quasi-isotropic)

\subsection{Discussion}

Existing couplings in a composite beam can cause undesirable deformations, which may in turn interfere with its normal performance. Examination of the laminate stiffness matrices (i.e. [A], [B], and $[\mathrm{C}]$ ) reveals that different types of coupling may occur in a composite laminate. In particular, when the elements $B_{16}$ and $B_{26}$ are zero in a composite laminate, in-plane normal force $N_{z}$ do not cause twist $\kappa_{z s}$ and nor does a twisting moment $M_{z s}$ cause elongation in the $z$-direction (i.e. $\varepsilon_{z}^{0}$ ). That said, a symmetric lay-up ensures that there is no extension and twist coupling in a composite laminate since in case of having angled plies (i.e., those at any angle other than $0^{\circ}$ and $90^{\circ}$ ), the 
twisting effect of such plies would be neutralized by those plies located at the same angle but on the other side of the neutral axis. However, in a composite beam with circular cross-section, this requirement is not sufficient to uncouple extension and twist in the beam structure since plies are located at different distances (with respect to the beam centroid). A circular CUS composite tube with a laminate containing plies in any angle other than $0^{\circ}$ and $90^{\circ}$ tends to twist when subjected to an axial load. To prevent the twisting effect of any ply at $+\beta$ (where $\beta$ is the angle between the fibre direction and the $z$-axis at any point on the wall), a ply of the same thickness wrapped at $-\beta$ is required but still not sufficient since the $-\beta$ ply would be located at a different radius. This indicates that having a balanced laminate does not guarantee the decoupling of extension and twist in a composite tube. The focus of this chapter was on composite tubes with circular crosssections due to their applications in biomedical implant (e.g. IM nail) design. In order to facilitate examining a composite tube for existing extension-twist coupling, a coupling factor $(\lambda)$ was employed which was a function of the laminate stacking sequence and the tube geometry provided that the thickness and the material properties of each ply would be the same. The factor can be used to examine the coupling in thin-walled as well as thick-walled composite tubes since the contribution of off cross-sectional strains to the beam deformation have been considered in the formulation. Examining the proposed factor in ten composite tubes with different lay-ups and investigating the extension-twist coupling in the same tubes using a 3D finite element model revealed interesting findings on laminated composite tubes structures.

Even though configuration $\mathrm{C} 1$ in Table 5-2 had a unidirectional laminate, it showed a noticeable coupling between extension and twist since applying a twisting moment of $100 \mathrm{~N} . \mathrm{m}$ led to an axial displacement of $0.99 \pm 0.008 \mathrm{~mm}$ in the plies forming the wall of the tube.

Examining configurations C2-C4 in Table 5-2 confirms the earlier statement that there could still exist an extension-twist coupling in hollow beams with balanced laminates. In other words, even though the elements $A_{16}$ and $A_{26}$ are zero in the laminates forming configurations $\mathrm{C} 2-\mathrm{C} 4$, which in turn uncouples extension and shear in the laminate, there still exists a coupling between extension and twist in the beam structure due to different distances of $+\beta$ and $-\beta$ from the centroid. Examining C5, one can conclude that unlike rectangular solid composite beams, hollow composite beams with a CUS structure can have extension-twist coupling while having a symmetric laminate. This is mainly due the fact that, in a CUS structure, $\beta(y)=\beta(-y)$ in which $y$ is measured from 
the centroid. As a result, the twisting effect of every angled ply would not simply be negated by the same ply on the opposite side of the centroid, whereas in a solid rectangular beam with a symmetric laminate, angled plies on both sides of the neutral axis would neutralize the twisting effect of each other. Having a cross-ply laminate (i.e. made of only $0^{\circ}$ and/or $90^{\circ}$ plies) can uncouple extension-twist in a composite beam as can be observed in configurations C6 and C7. In any case, having a zero factor of $\lambda$ can result in complete decoupling of extension and twist. In the case of a cross-ply laminate, the coupling factor $\lambda$ is zero since the coefficient $C_{12}$ is zero for all plies in the laminate. In all other cases, the coefficient $C_{12}$ would have equal magnitudes and opposite signs for plies places at $+\beta$ and $-\beta$ assuming identical thickness and material for all plies. The term $\left(R+h_{i}\right)^{2}-\left(R+h_{i-1}\right)^{2}$ takes into account the effect of each ply's location with respect to the centroid, and the summation of $\left(C_{12}\right)_{i}\left[\left(R+h_{i}\right)^{2}-\left(R+h_{i-1}\right)^{2}\right]$ over the laminate can indicate whether or not the $+\beta$ and $-\beta$ plies would neutralize the effect of one another in the laminate structure. Although the coupling factor $\lambda$ is zero for hollow beams with symmetric quasi-isotropic laminate (as in C8), this is not a mandatory condition to eliminate extension-twist coupling as one can see that $\mathrm{C} 9$ and $\mathrm{C} 10$ do not possess a quasi-isotropic laminate but are still free of extension-twist coupling.

\subsection{Summary}

In a circular composite tube with a CUS structure, where $x$-axis an $y$-axis lie in the cross-sectional plane, a bending moment in the $x$-direction does not cause a curvature in the $y$-direction and vice versa [123]. However, there exist a coupling between extension and twist in the beam. Such coupling can lead to undesired deformation of the structure which may interfere with its normal performance. In this chapter, a quantitative factor was introduced for extension-twist coupling in circular composite tubes. Given that the transverse shear effects and 3D elastic effects in the beam walls were considered in the derivation of the coupling factor, it can be used in both thin-walled and thick walled composite tubes. Moreover, this quantitative coupling factor can be used to examine the existence of extension-twist coupling, and also to compare the strength of such coupling in composite tubes. Validations were performed on ten circular composite tubes with different stacking sequences using a 3D finite element model which considered the interactions amongst plies and the out-of-plane displacement of the beam. Comparisons revealed that the 
coupling factor could accurately predict the extension-twist coupling in a composite tube with a circular cross-section. The presented factor could be used in the design process of structures containing circular composite tubes, in order to uncouple extension and twist. 


\section{OPTIMIZATION OF THE COMPOSITE IM NAIL STRUCTURE}

\subsection{Introduction}

The aim of this chapter was to optimize a composite (CF/epoxy) structure for use in IM nail applications based on the selective stress shielding approach. The optimization process was performed using equations derived in chapter 4 from the classical laminate theory and beam theory for composite hollow tubes. The mechanical performance of the nail in terms of fracture stability, load sharing, and strength in transverse and oblique shaft fractures was then investigated using an experimentally validated finite element model. Finally, a validated mechano-biochemical model for bone remodelling was employed to compare the long-term response of the bone to the conventional Ti-based alloy and composite IM nails.

\subsection{Optimization process}

The optimization process started with the identification of the major mechanical parameters influencing the rigidity and strength of the structure. CF/epoxy was used as the material of choice due to its superior strength and confirmed biocompatibility [64, 81]. Since IM nails have a hollow structure, the most important input parameters were the thickness and stacking sequence of the laminate. The output diameter of the nail was selected based on the femoral canal diameter and could not be altered as a design parameter. As such, this diameter was set to $14 \mathrm{~mm}$. The wall thickness, which depends on the number of layers of the laminate, was changed to customize the rigidity and strength of the structure. A range of 10 to 20 layers was considered in the optimization process, leading to a range of 1.56 to $3.12 \mathrm{~mm}$ for the wall thickness. A set of simulations showed that a thickness of less than $1.56 \mathrm{~mm}$ for the composite nail would result in failure under physiological loadings regardless of the stacking sequence. With regards to the angles, four different values were considered as possible orientations for each ply (i.e. $0,-45^{\circ}, 45^{\circ}$, and $90^{\circ}$ ). Taking into account the aforementioned orientations, all possible angular configurations for each thickness were obtained. The number of configurations varies based on the thickness. For instance, a thickness of $1.56 \mathrm{~mm}$ with 10 layers would have $4^{10}$ configurations, while a $3.12 \mathrm{~mm}$-thick laminate with 20 layers could be assembled in $4^{20}$ different configurations. 
The configurations were then filtered, and only those candidates which met the following criteria were considered:

1. Had no extension-twist coupling - this criteria required that the factor $\lambda$ be zero in the configuration. Full descriptions regarding the derivation and validation of this factor were included in Chapter 5.

2. Had a greater than $10 \%$ composition of $0^{\circ}$ and $90^{\circ}$ plies - minimized undesirable warping/curvature during the manufacturing process [103].

3. Had a $60 \%$ or less composition of plies in any direction-prevented the laminate from splitting parallel to the principal loading axis at screw holes [103].

4. Had at least two $90^{\circ}$ plies at the inner surface-increased laminate transverse rigidity while minimally affecting the bending and torsional rigidity.

All other configurations were excluded from the set, reducing the number of applicable configurations for each thickness. For instance, this number dropped from $4^{10}$ and $4^{15}$ to 11,327 and 9,189,467 for the $1.56 \mathrm{~mm}$-thick and the $2.34 \mathrm{~mm}$-thick nails respectively. The ultimate goal of the current optimization process was to maximize the bending and torsional rigidities of the nail while keeping it axially flexible, thereby minimizing the axial rigidity. Using a homemade computer code developed in MATLAB (Mathworks Inc., MA, USA), axial, bending, and torsional rigidities were obtained for the remaining configurations using the following equations:

$$
\begin{aligned}
& E A=2 \pi R \frac{1}{D^{*}}\left(\delta_{11}-\frac{\delta_{12}^{2}}{\delta_{22}}\right) \\
& E I=\pi\left(\frac{R^{\prime}}{\delta_{11}^{\prime}}+\frac{R^{\prime 3}}{\alpha_{11}^{\prime}}\right) \\
& G J=\frac{2 \pi R^{\prime \prime 3}}{\alpha_{66}^{\prime \prime}}
\end{aligned}
$$

where $R$ is the mid-surface radius of the nail, and $\alpha_{i j}$ and $\delta_{i j}$ are obtained from the inverse stiffness matrices of the laminate. Full descriptions and details concerning the derivation of rigidity formulae were explained in Chapter 4. Each configuration yielded a set of axial, bending, and torsional rigidities. There is no data in the literature regarding the required axial, bending, and 
torsional rigidities of an IM nail for a proper fracture healing. However, bending rigidity is believed to be the leading factor that influences the fracture stability in long bone fractures with internal fixation [84]. The bending rigidity of a conventional Ti-alloy nail is $132.8 \mathrm{~N} . \mathrm{m}^{2}$. Out of all possible combinations, those resulting in a bending rigidity lower than $40 \mathrm{~N} \cdot \mathrm{m}^{2}$ (30\% of that in the Ti-alloy nail) were excluded from the set of candidates. The remaining configurations were then categorized into sequentially increasing intervals of $10 \mathrm{~N} . \mathrm{m}^{2}$ based on their bending rigidity. Of the configurations in each interval, which differed by stacking sequence and thickness, the one with the lowest Stiffness Deviation Factor (SDF) was chosen. This factor is defined by:

$$
S D F_{i}=\left(\frac{E A_{i}-E A_{T i}}{E A_{T i}}\right)+\left(\frac{G J_{T i}-G J_{i}}{G J_{T i}}\right)
$$

where $E A_{i}$ and $G J_{i}$ are the axial and torsional rigidities of the $i^{\text {th }}$ configuration respectively, and $E A_{T i}$ and $G J_{T i}$ represent the axial and the torsional rigidities of a conventional Ti-alloy nail respectively. The $S D F$ describes how close each configuration in a given interval is to the ideal case, in which the laminate is rigid in torsion while axially flexible. Utilizing the aforementioned selection procedure, there were 7 candidates which passed to the second round (i.e. one from each interval of bending rigidity). For comparison purposes, a conventional IM nail made of Ti-alloy (Ti-6AL-4V) with an outer diameter of $14 \mathrm{~mm}$ and a thickness of $3.2 \mathrm{~mm}$ was also examined, leading to a total of 8 configurations.

\subsection{Finite Element Analysis}

A three dimensional model of a large left fourth-generation composite femur (model 3406, Sawbones, Vashon, WA, USA) was employed in the present chapter. The FE model of the bone was previously developed and validated by comparing its axial and torsional rigidities with those measured experimentally [119]. The new generation of synthetic bones have been shown to have no significant differences in elastic or failure properties when compared to cadaveric bone [134]. A $420 \mathrm{~mm}$ Stryker T2 femoral nail (Stryker, Mahwah, NJ, USA) was modeled and virtually implanted into the femur in accordance with the manufacturer's instructions using SolidWorks software (Dassualt Systèmes, Concord, MA, USA). The nail was initially modeled as a shell to ensure it would be capable of being used as a composite laminate later in simulations. The shell structure was later used as a reference and the nail was eventually meshed with solid elements. 
Four $5 \mathrm{~mm}$ locking screws were modeled as solid bodies and positioned to protrude approximately $2 \mathrm{~mm}$ out of the femur [5]. Assemblies were imported into ANSYS Workbench 15.0 software (ANSYS Inc., Canonsburg, PA, USA) to generate finite element (FE) models. ANSYS Composite PrepPost module was employed to assign the stacking sequence of the laminates in the composite IM nails selected in section 6.2. In this context, each layer was modeled using solid elements (i.e. SOLID185), and was assigned orthotropic material properties considering the ply angle. The Ti6AL-4V nail was also meshed with the same solid elements after assigning the thickness and converting it to a solid body. The number of body elements after convergence was 444,137 and the number of contact elements was 83,476 , leading to 527,613 elements in total in the FE model. The elastic material properties used in this chapter were tabulated in Table 6-1. The bone-nail assembly was studied at post-operative (PO) and healed bone (HB) stages.

Table 6-1. Linear elastic material properties used in this chapter [107-109]

$$
\begin{array}{cccccccccc}
E_{X} & E_{Y} & G_{X Y} & v_{X Y} & v_{Y Z} & \sigma_{X U} & \sigma_{Y U} & \sigma_{X U}^{\prime} & \sigma_{Y U}^{\prime} & \tau_{X Y U} \\
(G P a) & (G P a) & (G P a) & & & (M P a) & (M P a) & (M P a) & (M P a) & (M P a)
\end{array}
$$

\begin{tabular}{l|l|c|c|c|c|c|c|c|c|c|c|}
\hline \multicolumn{2}{|l|}{ Prepreg CF/epoxy* } & 121 & 8.6 & 4.7 & 0.27 & 0.4 & 2231 & 29 & -1082 & -100 & 60 \\
\hline \multicolumn{2}{|l|}{ Ti-6AL-4V } & 113.8 & - & - & 0.34 & - & 950 & - & - & - & - \\
\hline \multirow{2}{*}{ Bone } & Cortical & 16.7 & - & - & 0.26 & - & 106 & - & - & - & - \\
\cline { 2 - 12 } & Trabecular & 0.155 & - & - & 0.3 & - & 6 & - & - & - & - \\
\hline
\end{tabular}

*The layers were made of unidirectional (UD) and carbon sheets, with their $X$-axis aligned with the axis of the IM nail.

In the PO stage, mid-shaft femoral fractures at three different angles were considered (Figure 6-1). These included a transverse fracture, a $60^{\circ}$ proximal medial to distal lateral (PMDL) oblique fracture, and a $60^{\circ}$ proximal lateral to distal medial (PLDM) oblique fracture. The bone and the nail were assumed to have nonlinear frictionless contact[1][1][1][1]. The same type of contact was assumed at the fracture site to account for sliding, separation, and force transmission between fracture fragments. The screws were assumed to be bonded to the bone, simulating perfect 
interdigitation of the cortical bone with the threads of the screws, and the nail and the screws were assumed to have a no-separation contact in which sliding of the contacting surfaces is allowed. A total of seven parameters were employed during this stage to capture the biomechanics of fracture healing as in the author's previous studies $[114,135]$. They included the: compressive normal force at fracture, average von-Mises stress in the vicinity of the fracture site, fracture opening, shear movement at fracture, and the inverse safety factor (based on Tsai-Hill failure criterion) in the nail.

The distal epiphysis was fully constrained in all stages to avoid rigid body motion. In order to simulate physiological loading, the model was loaded with muscle and hip joint reaction forces corresponding to $45 \%$ of the gait cycle, as determined in previous studies $[110,112]$ as this instance of the gait produces maximum loads on the femur [121]. Five major muscles (i.e. m. gluteus minimus, m. gluteus medius, m. gluteus maximus, m. abductor magnus, and m. psoas) were considered in this muscle system. The muscle forces were applied to the femur as explained in section 3.2.3. 


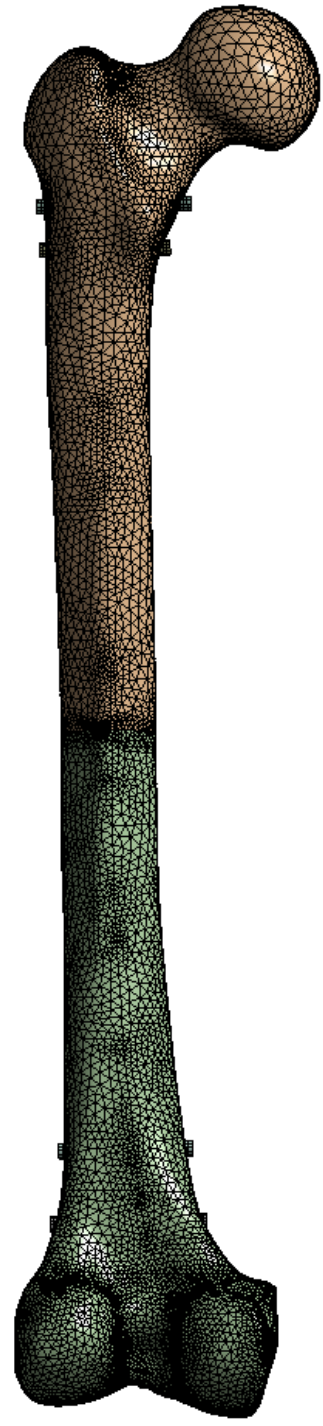

(a)

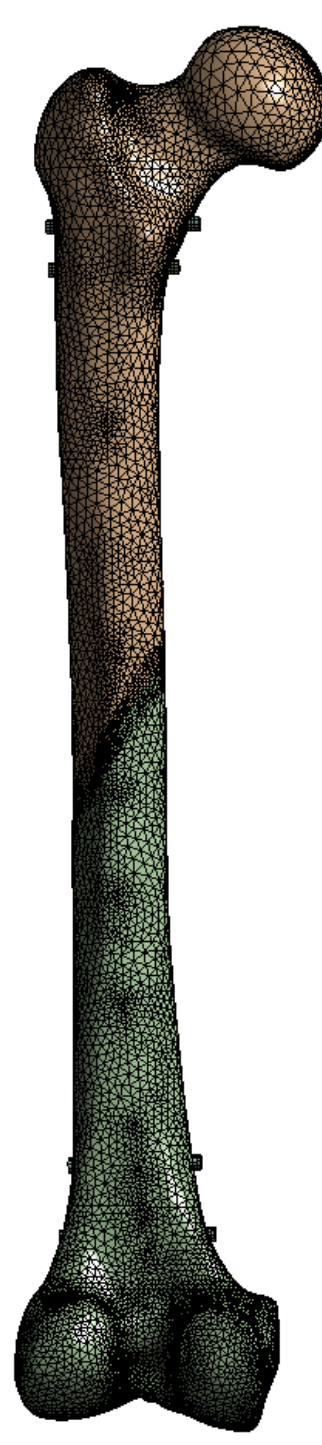

(b)

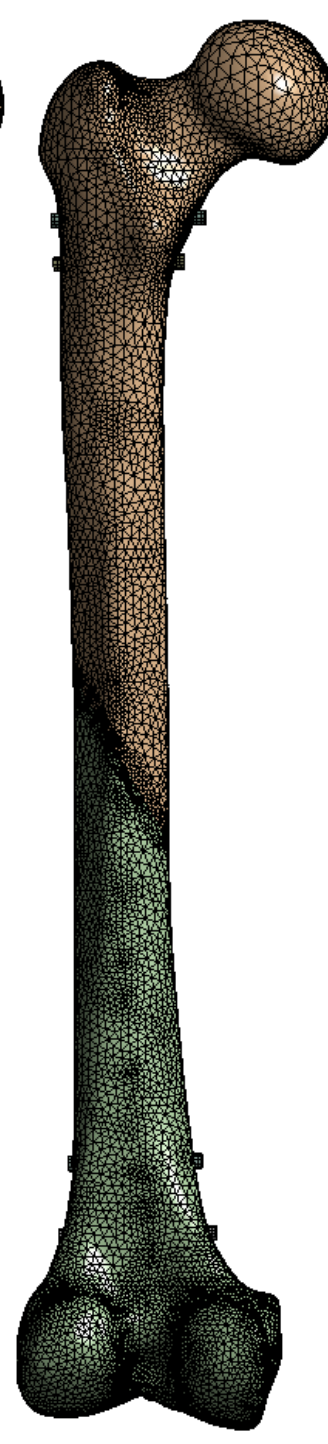

(c)

Figure 6-1. Finite element models of a femur with a transverse (a), proximal medial to distal lateral (PMDL) oblique (b), and proximal lateral to distal medial (PLDM) oblique (c) fracture, which was fixed with an IM nail.

\subsubsection{Mechano-biochemical (thermodynamic-based) model}

In the healed bone (HB) stage, the nail was supposed to minimally affect the loading of the bone and thus does not cause severe bone loss. To obtain the degree of bone loss in the femur associated with the internal fixation implant, a mechano-biochemical model was employed in this chapter. This bone remodeling model was previously validated by Tavakkoli Avval et al. [136]. Validation 
was performed by comparing the predicted long-term femoral density changes in response to total hip arthroplasty (THA) with those reported in a clinical study by Li et al. [137] who measured the periprosthetic changes in BMD in response to THA using DEXA. The bone remodeling model treated the bone as a thermodynamic system, exchanging energy matter and entropy with its surroundings. In this model, it was assumed that bone remodeling mechanisms were executed by bone resorption and formation phases through five biochemical reactions (i.e. formation of multinucleated osteoclasts, old bone decomposition, production of osteoblast activator, osteoid production, and calcification). The model assumed that the coupling between mechanical and biochemical fluxes or forces drives bone remodeling mechanisms. The details pertaining to the model were explained in a study by Tavakkoli Avval et al. [136].

The assumptions in the FE model in the HB stage were identical to those made in the PO stage except for those regarding the fracture contact, as bone continuity was assumed to be recovered at this stage.

\subsection{Results}

\subsubsection{Optimal configurations}

The best configuration in each interval of bending rigidity, identified as the one with the lowest SDF, along with their axial, bending, and torsional rigidities are tabulated in Table 6-2. 
Table 6-2. Optimal configurations in the intervals of bending rigidity. EA, EI, and GJ denote axial, bending, and torsional rigidities respectively.

\begin{tabular}{|c|c|c|c|c|c|}
\hline$\dot{\Xi}$ & Stacking Sequence & $\begin{array}{c}\text { EI } \\
\left(N . m^{2}\right)\end{array}$ & $\begin{array}{c}\text { EA } \\
(M N)\end{array}$ & $\begin{array}{c}\text { GJ } \\
\left(N . m^{2}\right)\end{array}$ & $\mathbf{S D F}^{*}$ \\
\hline C1 & {$\left[-45 / 45_{2} /-45_{2} / 45_{2} /-45_{2} / 45 / 0 / 45 /-45 / 0 / 90_{4}\right]$} & 39.9 & 3.31 & 83.4 & -0.35 \\
\hline $\mathrm{C} 2$ & {$\left[-45 / 45_{2} /-45 / 0 /-45 / 45_{2} /-45 / 0 / 45 /-45_{2} / 45 / 90_{4}\right]$} & 48 & 3.31 & 79.2 & -0.307 \\
\hline C3 & {$\left[-45 / 45 / 0_{2} / 45 /-45_{2} / 45_{2} /-45_{2} / 45_{2} /-45 / 90_{4}\right]$} & 55.3 & 3.31 & 75.9 & -0.274 \\
\hline C4 & {$\left[0_{2} /-45 / 45_{2} /-45_{2} / 45_{2} /-45_{2} / 45_{2} /-45 / 0 / 90_{2}\right]$} & 63.5 & 3.7 & 74.1 & -0.196 \\
\hline C5 & {$\left[0_{2} /-45 / 45_{2} /-45 / 0 /-45 / 45_{2} /-45_{2} / 45_{2} /-45 / 90_{2}\right]$} & 70.3 & 3.7 & 70.9 & -0.164 \\
\hline C6 & {$\left[0_{2} /-45 / 45 / 0 /-45 / 45_{2} /-45 / 0 /-45 / 45_{2} /-45 / 90_{2}\right]$} & 80.2 & 4.21 & 63 & -0.008 \\
\hline C7 & {$\left[0_{4} /-45 / 45_{2} /-45_{2} / 45_{2} /-45_{2} / 45 / 90_{2}\right]$} & 87.9 & 4.22 & 59.8 & 0.024 \\
\hline $\mathrm{C8}$ & Ti-6AL-4V & 132.8 & 6.7 & 98.9 & - \\
\hline
\end{tabular}

*SDF is calculated using Eq. (6.4).

\subsubsection{FEA results}

\section{PO stage}

Fracture opening and shear movement at the fracture site in transverse and oblique mid-shaft fractures fixed with metallic and composite IM nail candidates has been shown in Figure 6-2. The inverse safety factor (ISF) was measured at this stage to determine the susceptibility of the nail to failure (Figure 6-3). Moreover, the compressive normal force at the fracture site and the average von-Mises nodal stress in the vicinity of the fracture were also calculated, and have been depicted in Figure 6-4. The maximum von-Mises stress in the bone found in all fracture angles with the use of different composite IM nail configurations ranged from $42.5 \mathrm{MPa}$ to $57.9 \mathrm{MPa}$. 

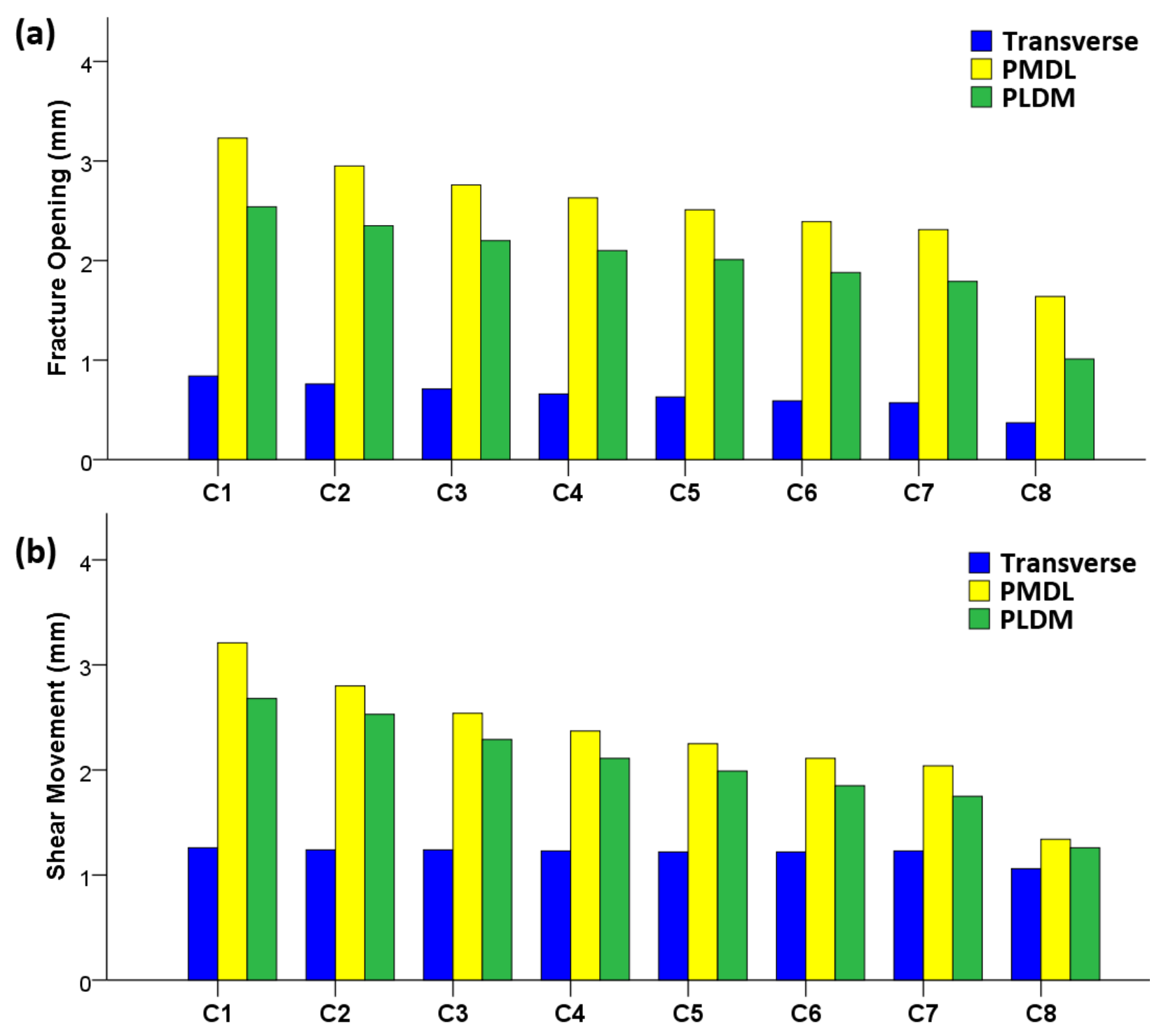

Figure 6-2. Fracture opening (a) and shear movement (b) at the fracture site for transverse and oblique mid-shaft fractures with the use of composite (C1-C7) and conventional metallic (C8) IM nails. 


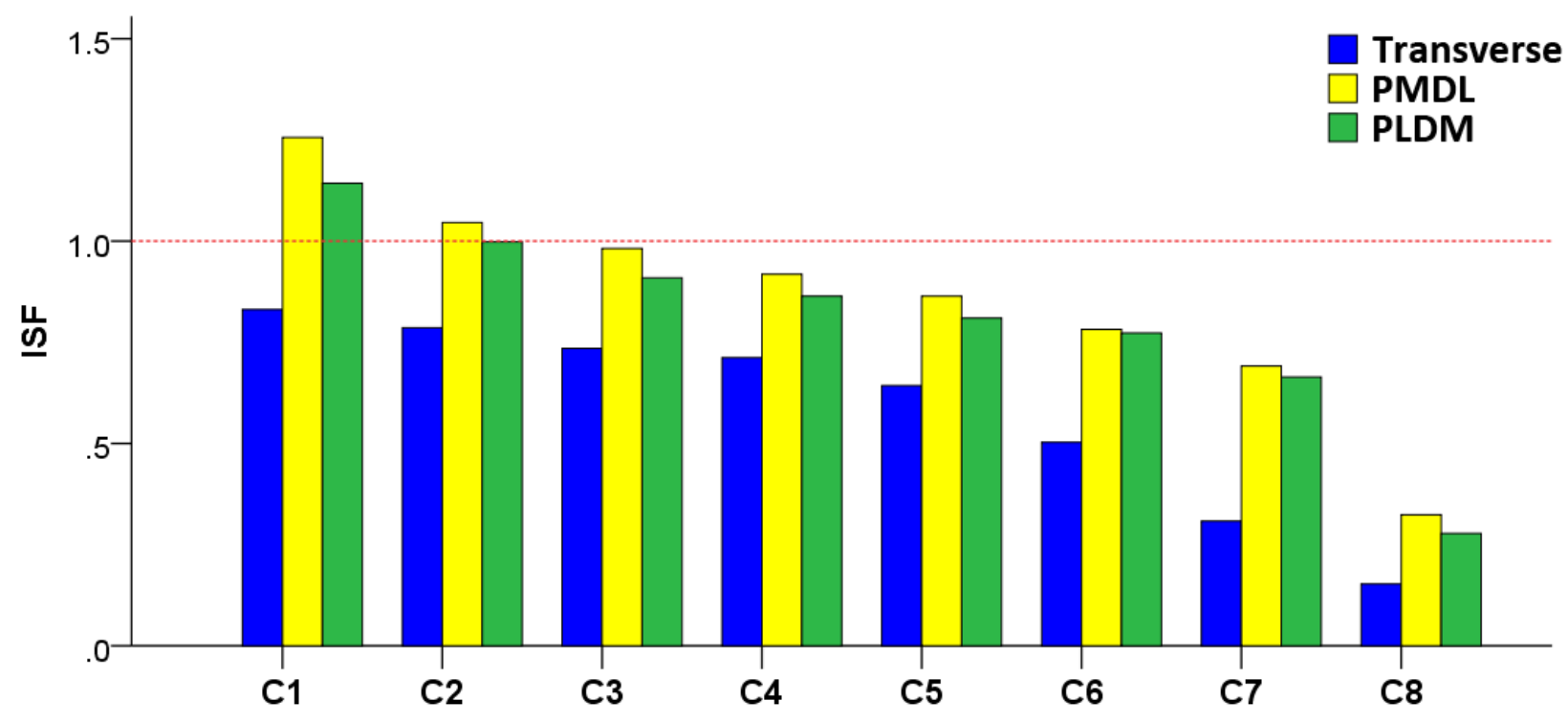

Figure 6-3. Nail inverse safety factor (ISF) for composite IM nail candidates (C1-C7) and the metallic IM nail (C8) in transverse and oblique mid-shaft femoral fractures. 


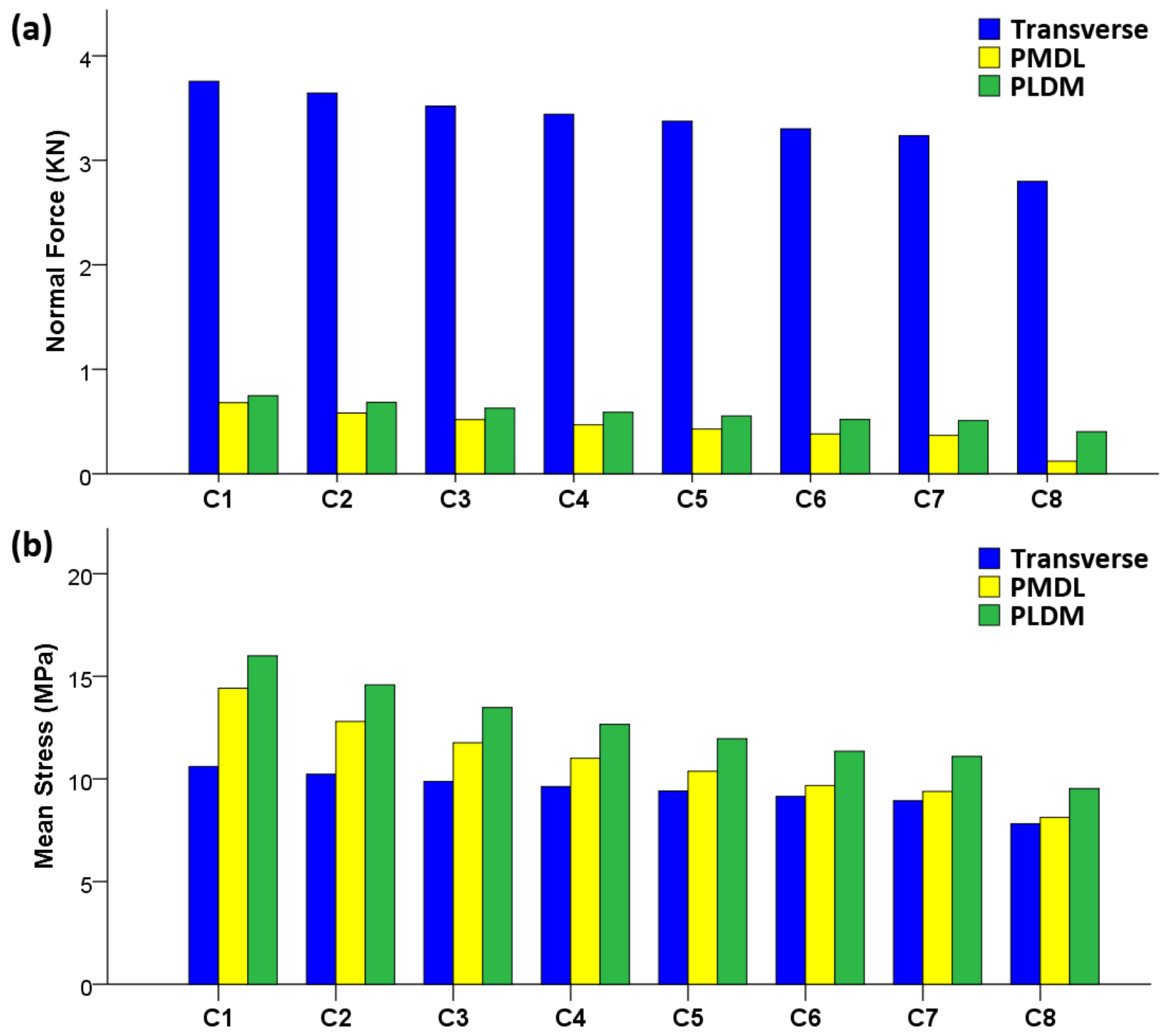

Figure 6-4. Compressive normal force at fracture (a) and average nodal stress in the bone at the vicinity of the fracture (b) for composite IM nail candidates (C1-C7) and the metallic IM nail (C8) in transverse and oblique mid-shaft femoral fractures.

\section{HB stage}

Healed bone results, simulating the long-term response of the bone, have been depicted in Figure 6-5a. Figure 6-5b illustrates the distribution of bone loss across a femur fixed with a typical composite IM nail candidate, in addition to a conventional Ti-alloy IM nail. 


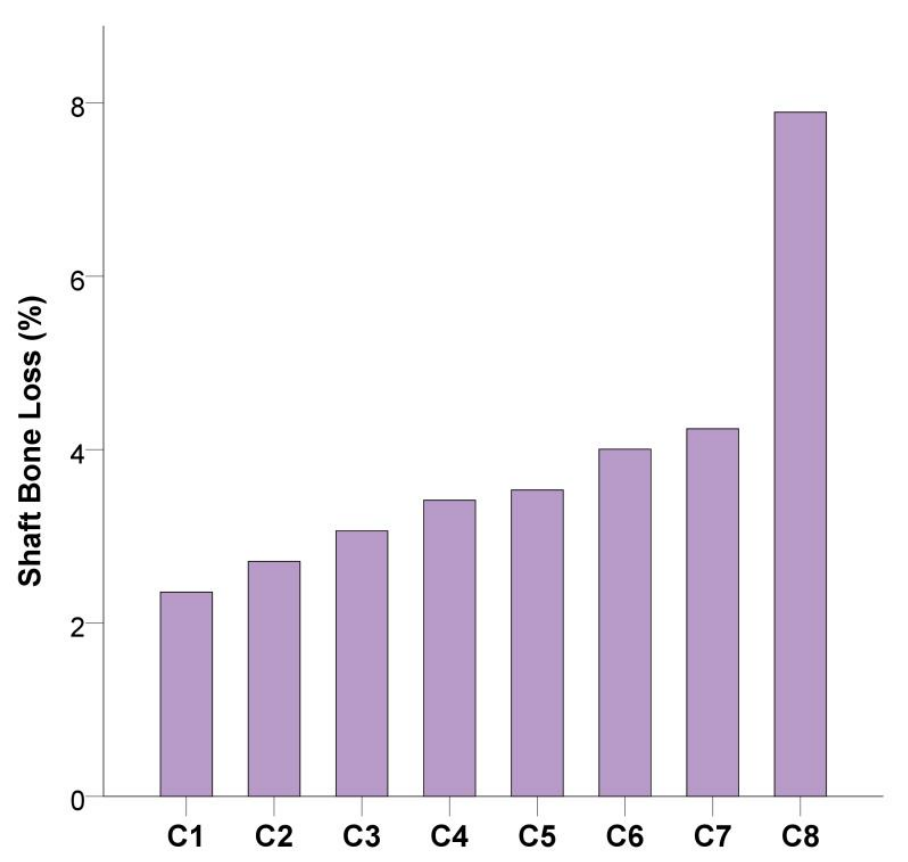

(a)

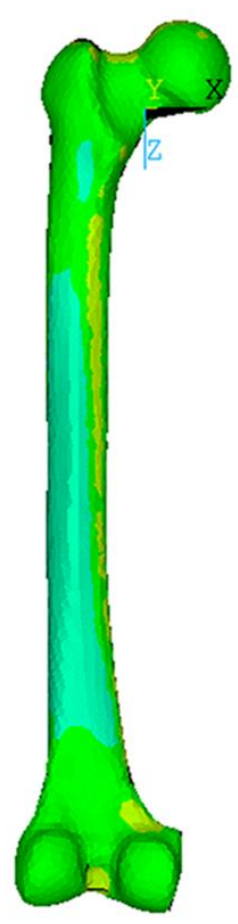

(b)

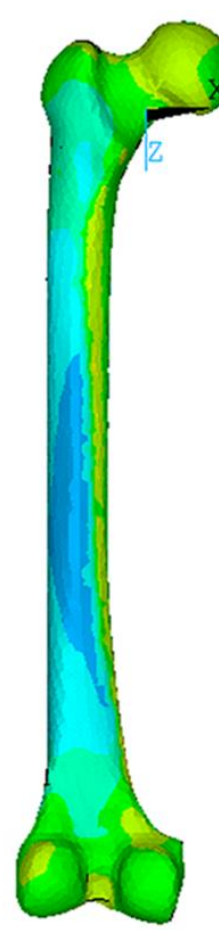

(c)

Figure 6-5. Long term average bone loss in the femoral shaft fixed with the composite IM nail candidates (C1-C7) and metallic IM nail (C8) (a). Percent change in femoral density in response to a typical IM nail candidate (b) as well as the metallic IM nail (c).

\section{Validating FEA with experimental measurements}

For the finite element results to be reliable, it is crucial to check the convergence of the solution (i.e. mesh sensitivity) and to verify the results against experimental data.

The convergence of mesh refinement was checked with "relevance" option in ANSYS Workbench with a value of -100 indicating a very coarse mesh and 100 corresponding to an extremely fine mesh. A set of simulations showed that a value of 75 will result in convergence and further increasing the relevance will not change the strain values more that $1 \%$.

Experimental result from Bougherara et al. [56] were used to verify the current simulation results since the same IM nail and composite femur were used in their mechanical testing (Figure 6-6). Axial stiffness as well as strain values at 5 different locations on medial surface were obtained for titanium nail in HB stage and compared to their measurements. An axial load of $580 \mathrm{~N}$ was applied on the femoral head to mimic their experimental test setup. Moreover, since they have used the $3^{\text {rd }}$ generation composite femurs in their study, the material properties of the current model were 
changed to those of the $3^{\text {rd }}$ generation bone. It is worth noting that the $3^{\text {rd }}$ generation and $4^{\text {th }}$ generation composite femurs (used in the current thesis) are completely identical in terms of geometry and that their only difference is their mechanical properties. The average displacement of the nodes on femoral head in the direction of the applied load was used to obtain the stiffness of the structure.

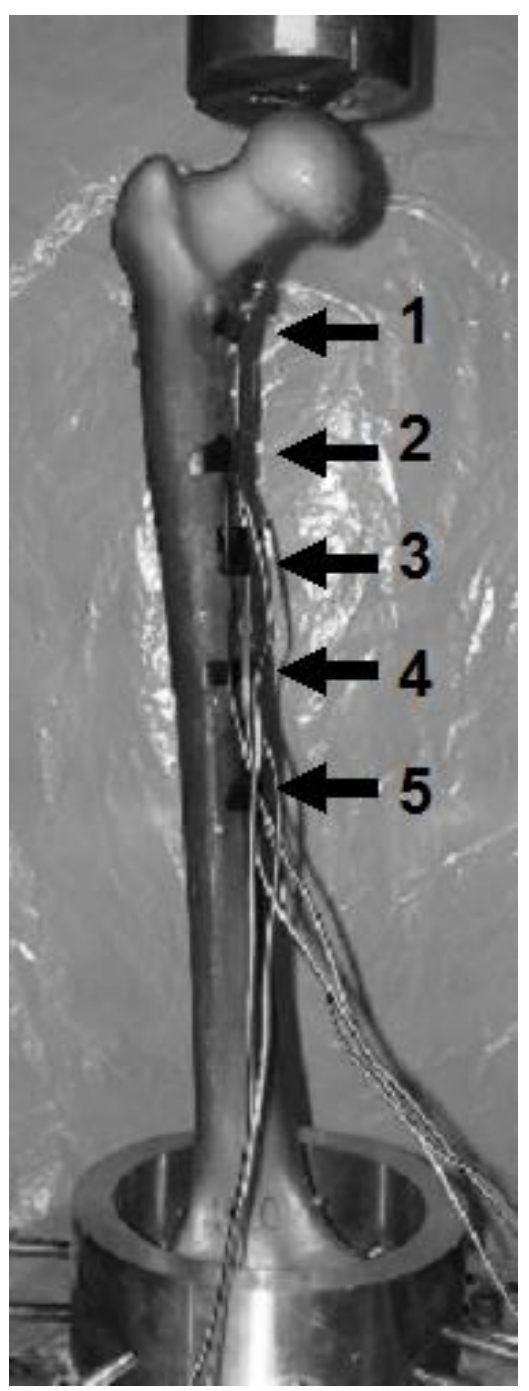

Figure 6-6. Experimental setup used by Bougherara et al. [56] to validate FE results.

The HB results were compared to similar studies in the literature $[56,119]$. FE strain readings taken at the same strain gage locations as experimentally (Table 6-3). A good correlation $\left(\mathrm{R}^{2}=\right.$ 0.926) was found between the strain values obtained from the current FE model and those experimentally measured by Bougherara et al. [56]. The obtained stiffness (1378.8 N/mm) also 
compares favourably with that reported by the prior investigation for retrograde IM nail implantation on synthetic femurs $(1168.8 \mathrm{~N} / \mathrm{mm})$ [56], and also with those reported for intact synthetic femurs $(1290 \pm 30 \mathrm{~N} / \mathrm{mm})$ [119]. The slightly higher stiffness found in the current study compared to that reported by Bougherara et al. [56], which resulted in lower strain values at the 5 locations, might be due to the load application method (i.e. the flat plate) used in the experiment and the difference in the nail thickness in the IM nails used in the two studies.

Table 6-3. Results showing axial stiffness and microstrain values at different locations of the structure at HB stage. L1-L5 refers to location 1-5 as in Bougherara et al. [56].

\begin{tabular}{ccc}
\hline & $\begin{array}{c}\text { Current } \\
\text { chapter }\end{array}$ & $\begin{array}{c}\text { Bougherara et al. } \\
{[\mathbf{5 6}]}\end{array}$ \\
\hline L1 Strain & 817 & 856 \\
L2 Stain & 708 & 904 \\
L3 Stain & 531 & 647 \\
L4 Stain & 441 & 470 \\
L5 Stain & 300 & 309 \\
$\mathbf{R}^{\mathbf{2}}$ & & \\
\hline $\begin{array}{c}\text { Axial stiffness } \\
\text { (N/mm) }\end{array}$ & 1378.8 & \\
\hline
\end{tabular}

\subsection{Discussion}

\subsubsection{General findings}

In this chapter, a fibre reinforced composite IM nail made of CF/epoxy was optimized to obtain the lowest axial rigidity with the highest bending and torsional rigidities by altering the stacking sequence and the thickness of the laminate. The findings in Table 6-2 revealed that, in contrast to metallic nails, increasing the bending rigidity in a composite nail does not necessarily result in an increase in its axial rigidity. It is interesting to note that candidates $\mathrm{C} 1-\mathrm{C} 3$ yielded the same axial 
rigidity but differing bending rigidities. This enables one to tailor the rigidity based on fracture fixation requirements. The table also shows that the axial rigidity of the nail grows as the number of $0^{\circ}$ plies increases. Therefore, one may place the existing $0^{\circ}$ plies in a laminate closer to the outer surface to increase the bending rigidity, while minimally affecting the axial rigidity. When considering column GJ in Table 6-2, it can be concluded that the torsional rigidity of the nail is highly dependent upon the number of $45^{\circ}$ plies and their proximity to the outer surface. That is said, while use of additional $45^{\circ}$ plies favourably increases the torsional rigidity of the nail, it tends to simultaneously sacrifice the bending rigidity of the structure. It is worth mentioning that PMDL oblique fractures were found to be more critical when compared to transverse or PLDM oblique fractures, regardless of the laminate configuration, as they resulted in larger gap opening and shear movements, and lower compressive forces and average stress at the fracture site. The nail was also found to be more susceptible to failure due to a higher ISF. The lower stability of oblique fractures compared to transverse fractures was previously shown in a canine study [117].

\subsubsection{Selection of the best candidate}

FE results were used to evaluate the performance of each candidate under actual boundary conditions. The range of the maximum von-Mises stress in the bone (42.5 MPa to 57.9 $\mathrm{MPa}$ ) was found to be much lower that the cortical bone strength ( 106 MPa). As depicted in Figure 6-3, candidates $\mathrm{C} 1-\mathrm{C} 3$ resulted in an ISF of 1 or higher, indicating a vulnerability to failure. Thus, strength-wise, configurations C4-C7 could all be acceptable. These candidates also resulted in comparable gap openings and shear movements to those of a Ti-alloy nail. However, as seen in Figure 6-4 and Figure 6-5, as the bending rigidity of the nail increases, the factors deemed to be beneficial to fracture healing drop. Therefore, C5 was selected as the best candidate because in addition to its low axial rigidity $(3.70 \mathrm{MN})$, it had moderate bending and torsional rigidity (70.3 $N . m^{2}$ and $70.9 N . m^{2}$ respectively). Furthermore, it showed a significant increase in the amount of compression between fracture ends and the stress level in the vicinity of the fracture site, whilst still being sufficiently strong fixation structure for femoral diaphyseal fracture.

\subsubsection{Comparison of the best composite candidate with a conventional Ti-alloy nail}

The Ti-alloy IM nail had an axial rigidity of $6.7 \mathrm{MN}$, a bending rigidity of $132.8 \mathrm{~N} \cdot \mathrm{m}^{2}$ and a torsional rigidity of $98.9 \mathrm{~N} . \mathrm{m}^{2}$. The selected composite nail (C5) was $44.7 \%$ less stiff axially 
when compared to a conventional Ti-alloy nail. Furthermore, despite being $28.3 \%$ less rigid in torsion and $47 \%$ less rigid in bending, it would still provide sufficient stability for all transverse and oblique fractures, as shear movement at the fracture site increased by only $0.16 \mathrm{~mm}, 0.91 \mathrm{~mm}$, and $0.73 \mathrm{~mm}$ in transverse, PMDL, and PLDM oblique mid-shaft fractures respectively. It is worth mentioning that for oblique fractures, both torsional and bending rigidities that define the degree of shear movement. The proposed configuration would increase the compressive normal force between the fracture fragments by $20.6 \%(575.9 \mathrm{~N}), 255 \%(307.9 \mathrm{~N})$, and 37.5\% $(151.0 \mathrm{~N})$ when compared to a conventional Ti-alloy nail in transverse, PMDL oblique and PLDM oblique fractures respectively in the PO stage. In oblique fractures, a more flexible nail would maintain contact between the fragments, which would in turn increase compression.

The HB stage reflects the condition in which union has occurred and the implant is expected to minimally alter the amount and pattern of the loads carried by the bone. The main concern with the use of rigid implants is long-term bone loss resulting from the removal of physiologic loads from the bone. Examining Figure 6-5, one can see that implantation of the Ti-alloy nail results in an average bone loss of $7.9 \%$ in the femoral shaft while the selected composite nail reduces the bone loss to $3.5 \%$ in the same region. The value predicted by the mechano-biochemical model as the average bone loss due to the long-term presence of Ti-6Al-4V nail is in the range of those reported by Wang et al. [138] (1\% to $21 \%$ bone loss in the zones enveloping the mid-shaft). Additionally, in a clinical study by Allen et al. [139], it was reported that in response to the longterm (29 months) presence of an IM nail, BMD in a human tibia treated with a retained reamed nail decreased by $7 \%$ compared to that in the intact contra-lateral tibia which is consistent with our results.

\subsubsection{Potential limitations}

The present chapter has certain limitations. Firstly, the performance of the proposed IM nail was evaluated in mid-shaft femoral fractures only. Further investigations are therefore required before it can be used in other femoral fracture types or other diaphyseal fractures. Secondly, the composite nail design was evaluated in a single loading scenario ( $45 \%$ of the gait cycle). Due to the repetitive nature of physiological loads, the fatigue behaviour of the proposed design should be evaluated, which could be the focus of future studies. The mechano-biological model has also some limitations and simplifications. The concept of coupling is based on linear non-equilibrium 
thermodynamics and thus is phenomenological. Therefore, it cannot be directly related to the actual mechanosensing or mechanotransduction processes in cells. Additionally, the mechanical stimuli in the simulations do not include any viscous effects. It was also assumed that the trace of strains regulates bone cells response. However, other mechanical stimuli such as fluid flow and transport of nutrients were considered. In the proposed model, the computational steps were unrelated to real-time processes. Nonetheless, convergence of the iterative process was assumed when no significant change in the density of the elements was observed which, from a clinical point of view, is considered as the long-term response when the femoral density attains a stable condition. Finally, the in-vivo performance of the proposed composite nail must be evaluated using animal studies and ultimately clinical trials before it can be used as an alternative to conventional metallic nails.

\subsubsection{Clinical implications}

Several clinical investigations have shown reduction in bone mineral density due to stress shielding around IM nails [139-141]. A clinical study demonstrated that retained IM nailing results in statistically significant bone loss in the healed tibia [139]. Another clinical follow up by Eyres et al. [140] on five patients with mid-shaft tibial fracture reported significant bone loss 5-11 year after the time of fracture. Bone loss can be associated with a risk of re-fracture [139]. The proposed composite IM nail can significantly reduce the amount of bone loss by minimally affecting the strain pattern in the bone. Moreover, a flexible fixation device which stabilises the fracture can boost the fracture healing process. In an animal study, Sha et al. [60] showed that a flexible nail made of Ti-alloy exhibits better callus formation and reduces the stress shielding effect when compared to a more rigid nail. The current findings suggest an increase in the axial compression across the fracture site and in the average stress in the vicinity of the fracture when a more flexible nail is used for fixation purposes, which is consistent with the findings of previous studies [59, 142]. There are numerous studies which have considered flexible nailing in pediatric femoral shaft fractures [143-145] but only a few studies have investigated flexible nailing in diaphyseal fractures in adults [114], mainly due to insufficient stability of the fracture and increased risk of failure associated with those flexible implants.

This chapter suggests a number of guidelines when designing composite IM nails and is unique in a sense that it is the first to consider the optimization of a composite IM nail based on the selective 
stress shielding approach. The new methodology could potentially be used to improve the design of IM nails for diaphyseal fracture fixation.

\subsection{Summary}

This chapter introduced a new approach for the optimization of a fibre-reinforced composite nail made of carbon fibre/epoxy based on a combination of the classical laminate theory, beam theory, finite element method, and bone remodeling model using irreversible thermodynamics. The optimization began by altering the composite stacking sequence and thickness to minimize axial rigidity, while maximizing torsional rigidity for a given range of bending rigidities. The selected candidates for the 7 intervals of bending rigidity were then examined in an experimentally validated finite element model to evaluate their mechanical performance in transverse and oblique femoral shaft fractures. It was found that the composite nail having an axial rigidity of $3.7 \mathrm{MN}$, and bending and torsional rigidities of 70.3 and $70.9 \mathrm{~N} . \mathrm{m}^{2}$ respectively showed an overall superiority compared to the other configurations. It increased compression at the fracture site by $344.9 \mathrm{~N}$ on average, while maintaining fracture stability through an average increase of only 0.6$\mathrm{mm}$ in fracture shear movement in transverse and oblique fractures when compared to a conventional titanium-alloy nail. The long-term results obtained from the bone remodeling model suggest that the proposed composite intramedullary nail reduces bone loss in the femoral shaft from $7.9 \%$ to $3.5 \%$ when compared to a conventional Ti-alloy IM nail. This chapter also proposed a number of practical guidelines for the design of composite IM nails. 


\section{BIOMECHANICAL TESTING OF CARBON-FIBRE/EPOXY COMPOSITE IM NAIL}

\subsection{Introduction}

The purpose of this chapter was to determine the most influential mechanical properties of the $\mathrm{CF} /$ epoxy composite IM nail whose structure was previously customized to fulfill fracture healing requirements. In particular, the composite nail was tested in tension, compression, 3-point bending, and torsion to simulate clinical-type loading the nail is subjected to when placed in the femoral canal. In addition, the moisture absorption behavior of the composite nail was investigated to seek the possibility of using such structure in-vivo, and the surface hardness was obtained through the Rockwell Hardness B test. Finally, the response of the CF/Epoxy composite IM nail to cyclic loading was investigated through fatigue testing combined with infrared thermography analysis. It was hypothesized that the composite nail would have a high cycle fatigue strength (HCFS) that is much higher than the clinical-type loads experienced by an IM nail in physiologic activities.

\subsection{Methodology}

\subsubsection{Composite IM nail structure}

The composite IM nail was manufactured as a circular tube with an outer diameter of $14 \mathrm{~mm}$, which was selected based on the femoral canal diameter, using 17 plies. The stacking sequence of the laminate was selected as $\left[0_{2} /-45 / 45_{2} /-45 / 0 /-45 / 45_{2} /-45_{2} / 45_{2} /-45 / 90_{2}\right]$, with $0^{\circ}$ plies as the outermost plies. This stacking sequence was previously obtained through a comprehensive optimization process consisting of the classical laminate theory, beam theory, finite element method, and an irreversible thermodynamic model, as outlined in Chapter 6 and in one of the author's previous studies [146]. The composite tubes were manufactured from standard $24 \mathrm{~K}$ carbon-fibre/epoxy prepreg sheets (35\% resin content) by wrapping the prepregs around a mandrill and then oven curing the assembly at $135{ }^{\circ} \mathrm{C}$ for almost 1 hour. The inner diameter of the tubes was approximately $8.7 \mathrm{~mm}$. However, exact dimensions were measured for each specimen prior to testing. 


\subsubsection{Void fraction measurement}

In order to evaluate the quality of the consolidated structure and the porosity of the laminate, four specimens, each 1 in $(25.4 \mathrm{~mm})$ long, were cut randomly out of different manufactured tubes, resulting in eight surfaces to examine. The cutting surfaces were polished and examined under an optical microscope. Four images were taken of each cutting surface (one of each quadrant) yielding a total of 32 images. The images were then converted to 8-bit grey scale images and then the porous regions were identified as black islands using a threshold function in ImageJ software (National Institute of Health, USA) (Figure 7-1). The void percentage in the laminate was calculated by the ratio of dark contrast area to the total area in each image.

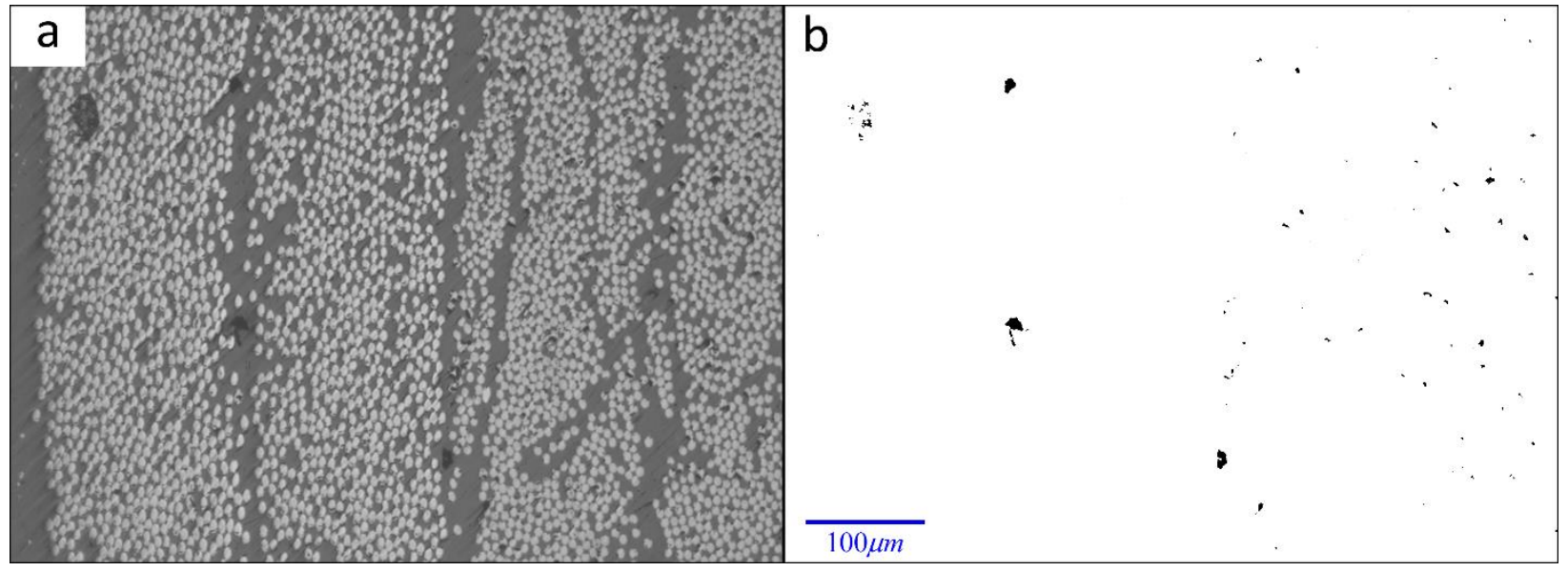

Figure 7-1. (a) A typical microscopic image of a specimen's cross-section used to calculate the porosity. (b) Estimated black porous areas of the cross-section in ImageJ software.

\subsubsection{Moisture absorption tests}

Standard saturated moisture absorption tests were conducted in accordance with ASTM D570 standard [147]. Five composite tubes, each $25.4 \mathrm{~mm}$ long, were cut randomly from manufactured specimens. The specimens were dried in an oven for $1 \mathrm{~h}$ at $110^{\circ} \mathrm{C}$ and immediately weighed to the nearest $0.001 \mathrm{~g}$ using a precision balance (Sartorius M-prove series, Sartorius Inc., NY, USA). They were then entirely immersed in distilled water maintained at room temperature. At the end of every $24 h r$ period, $+1 / 2$, the specimens were removed from the water and wiped off one at a time with a low lint cloth and weighed immediately to the nearest $0.001 \mathrm{gr}$ and then replaced in the water. Percentage increases in weight during immersion were calculated to the nearest $0.01 \%$ as follows [147]: 


\section{Increase in weight, $\%=\frac{\text { wet weight }- \text { conditioned weight }}{\text { conditioned weight }}$}

\subsubsection{Compression tests}

Compression tests were performed on specimens in accordance with ASTM standard for compressive properties of rigid plastics [148]. Five specimens, each $25.4 \mathrm{~mm}$ long, were cut randomly from the manufactured tubes using a table saw. The specimens were placed between two compression plates on a testing machine (STM series, United Calibration Corp., Huntington Beach, CA) with a $50 \mathrm{kN}$ load cell (Figure 7-2a). The testing speed was set to $1.3 \mathrm{~mm}$ for the whole duration of testing. The compressive modulus of elasticity was calculated by applying linear regression to the linear portion of the stress-strain curve. The ultimate compressive strength was determined by dividing the maximum compressive load carried by the specimen during the test by the original cross-sectional area. The failed specimens were then cut along the cylindrical axis and the cutting surfaces were examined.

\subsubsection{Tension tests}

Tubes were cut into pieces of $250 \mathrm{~mm}$ in length using a table saw based on recommended dimensions from the ASTM standard for tensile properties of polymer-matrix composites [149]. The ends of specimens had to be gripped firmly between the jaws of the testing machine. For this purpose, v-shaped grips were used to grab the specimens. In order to prevent crushing the specimens and to produce a state of uniform tension within the gauge length, two rigid plugs made of stainless steel were inserted in the ends as suggested by ASTM [150]. A tensile machine (STM series, United Calibration Corp., Huntington Beach, CA) with a constant head speed of $2 \mathrm{~mm} / \mathrm{min}$ and a $50 \mathrm{kN}$ load cell was employed to perform the experiments (Figure 7-2c). An extensometer (United Calibration Corp., Huntington Beach, CA, USA) was placed at the middle of the gripped specimens to measure elongation. Five specimens were loaded to failure at room temperature and load and elongation were recorded simultaneously by the testing machine. Tensile modulus of elasticity, obtained by applying linear regression to the linear portion of the graph, and ultimate tensile strength of the specimens, determined by dividing the maximum load by the specimen cross-sectional area, were calculated for each specimen. Mean value and standard deviation of the 
obtained were then calculated. The failed specimens were cut along the cylindrical axis and the cutting surfaces were examined to investigate the failure mode.

\subsubsection{Bending tests}

Five specimens with a length of $250 \mathrm{~mm}$ were tested in three-point bending according to the guidelines provided by ASTM [151]. The testing was performed on the same testing machine used for tension and compression tests. A standard fixture with a span length of $230 \mathrm{~mm}$ was mounted on the testing machine to support the specimens (Figure 7-2b). The specimens were loaded at the middle of the supports through a loading nose at a constant speed of $1 \mathrm{~mm} / \mathrm{min}$. The flexural modulus of elasticity and the ultimate flexural strength were calculated using Eqs. (7.2) and (7.3) respectively which were taken from ASTM [151] and were modified for circular tubes.

$E_{f}=\frac{4 M L^{3}}{3 \pi\left(d_{o}^{4}-d_{i}^{4}\right)}$

$\sigma_{\max }=\frac{8 d_{o} F_{\max } L}{\pi\left(d_{o}^{4}-d_{i}^{4}\right)}$

where $M$ is the slope of the load deflection curve obtained from the linear portion of the graph, $L$ is the support span, and $d_{o}$ and $d_{i}$ are the outer and inner diameters of the tube.

\subsubsection{Torsion tests}

Five specimens with an overall length of $225 \mathrm{~mm}$ were tested in a torsion testing machine (CCT series, Shenzhen Sans Testing Machine Co. Ltd., Shenzhen, China) with a maximum twisting moment of $200 \mathrm{~N} . \mathrm{m}$. The testing procedure was in accordance with the ASTM standard guidelines for standard torsion test [152]. Two aluminum discs were attached to the tubes using custom-made semi-rings making a gauge length of $100 \mathrm{~mm}$, and were used to measure the twist angle (Figure 7-2d). The torsion tests were performed at a constant speed of $15 \mathrm{deg} / \mathrm{min}$ and the specimens were loaded up to failure. The shear modulus and the shear strength were calculated using the following equations [152]:

$G=\frac{32 m l}{\pi\left(d_{o}^{4}-d_{i}^{4}\right)}$ 
$\tau_{\max }=\frac{16 d_{o} T_{\max }}{\pi\left(d_{o}^{4}-d_{i}^{4}\right)}$

in which $m$ is the slope of the twisting moment versus twist angle curve obtained from the linear portion of the graph, $l$ is the gauge length, and $d_{o}$ and $d_{i}$ are the outer and inner diameters of the tube.

\subsubsection{Rockwell hardness tests}

To measure the surface hardness of the specimens, Rockwell B hardness tests were conducted pursuant to ASTM Standard Test Method for Rockwell Hardness of Plastics and Electrical Insulating Materials [153]. This standard was chosen since there was no ASTM standard for Rockwell hardness test of polymer-matrix composites [81]. Eight specimens were cut from composite tubes in 1 in $(25.4 \mathrm{~mm})$ pieces. Each specimen was supported by the same steel plug which was used in tension tests, and the convex surface of the specimen was loaded by a steel ball with a 1/16 in diameter at a load of $100 \mathrm{~kg}$. Fifteen readings were performed on each sample and the mean value for these readings was considered as the hardness of each specimen. Tests were conducted at ambient room temperature using a Rockwell hardness tester (LR300TD, LECO Corporation, St. Joseph, MI, USA) (Figure 7-2e). The readings were corrected by a correction factor for convex surfaces according to the chart provided by the manufacturer.

\subsubsection{Surface roughness measurements}

Surface roughness of the composite tubes was measured by profilometry using a NANOVEA ST400 non-contact optical profilometer (Micro Photonics Inc., Allentown, PA, USA) with a 150$\mathrm{mm} \mathrm{X}-\mathrm{Y}$ stage. Measurements were made on three specimens on $5 \mathrm{~mm} \times 1 \mathrm{~mm}$ regions with $10 \mu \mathrm{m}$ step size. The average surface roughness $\left(R_{a}\right)$ was then obtained along three individual paths along the fibres direction and perpendicular to them. 

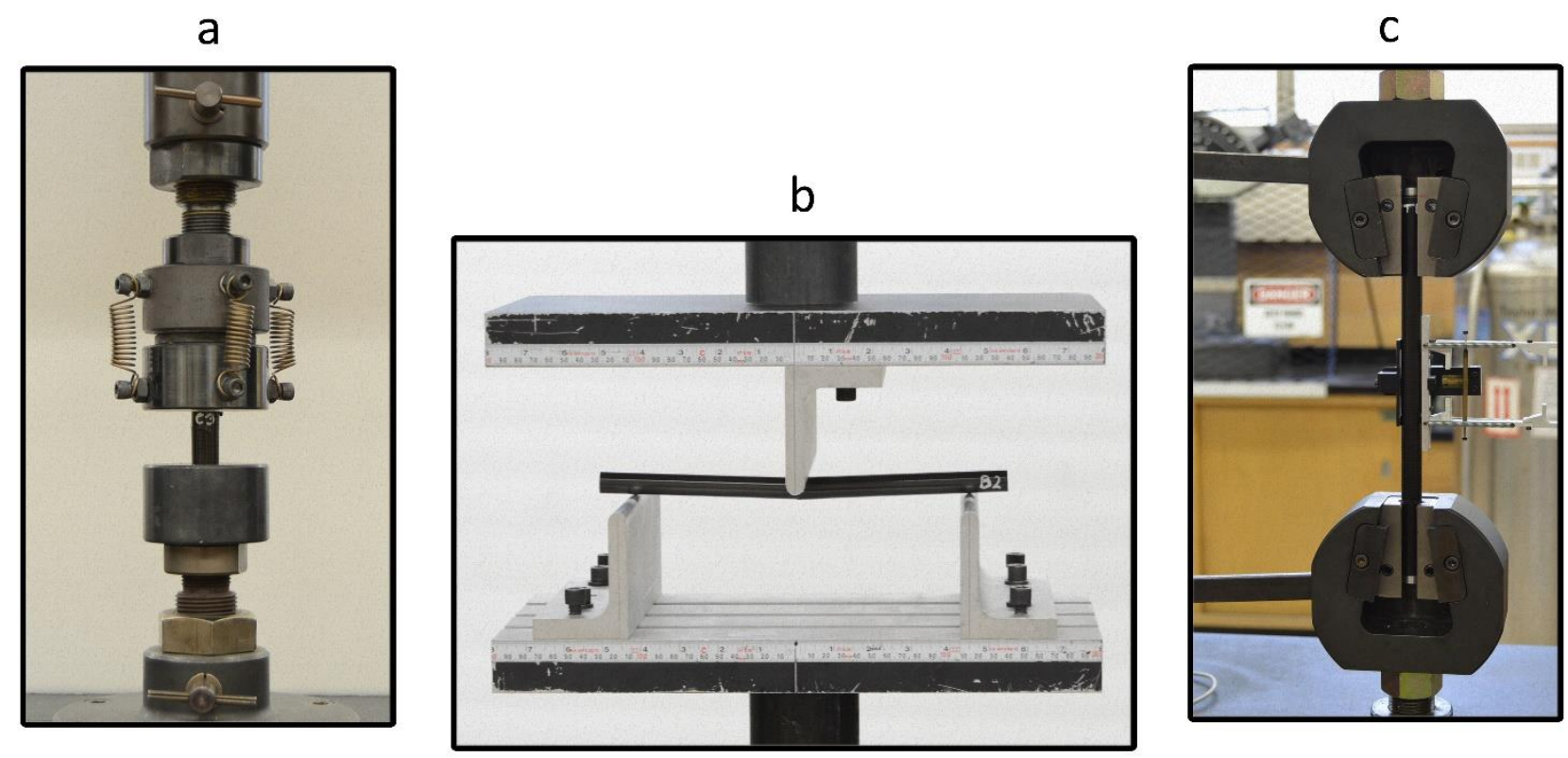

d

e
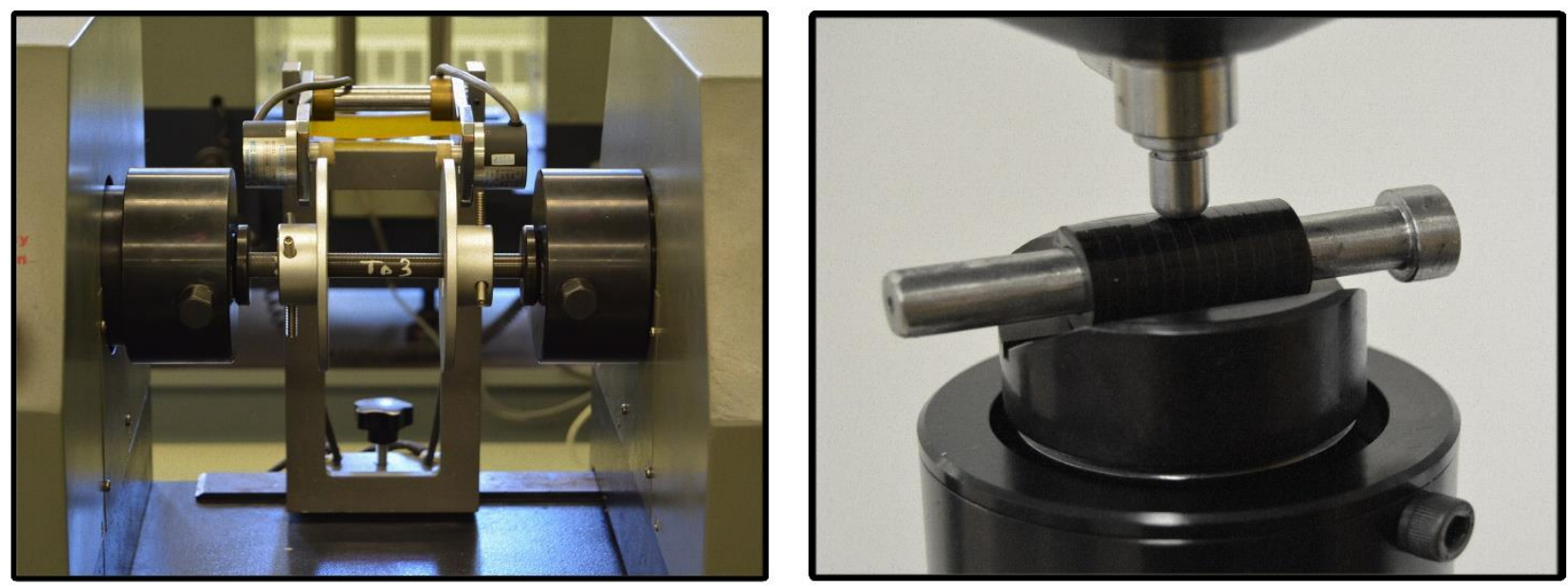

Figure 7-2. Quasi-static mechanical tests set up: (a) compression test (b) bending test (c) tension test (d) torsion test (e) Rockwell Hardness test. 


\subsubsection{Fatigue tests}

\section{Test setup}

Fatigue tests were performed on the CF/epoxy IM nails to determine the fatigue strength of the nail structure under clinical-type cyclic loading condition. Infrared thermography (IRT) approach was used rather than conventional fatigue testing approach to determine the HCFS (high cycle fatigue strength) of the composite nails. The main power point of using this technique is that the HCFS of the material can be obtained by testing few specimens in a few hours with sufficient accuracy [154]. Thermography is a measurement technique that involves the use of an infrared (IR) camera to provide a time-dependant contour map of the specimen surface temperature. A similar approach to that used by La Rosa et al. [155] to find the fatigue limit of various metallic alloys and was later used and validated by Montesano et al. [154] to determine the HCFS of carbon fibre-reinforced composites was followed in this chapter. In this approach, the energy dissipated at various stress levels was monitored by measuring the difference in surface temperature. This difference was an increasing function of the applied stress amplitude, which was initially slow and then suddenly accelerated. The fatigue limit was found by this thermomechanical threshold.

Tests were performed on three specimens, each $250 \mathrm{~mm}$ long using a servo-hydraulic testing machine (Model 322, MTS System Corporation, Eden Prairie, MN, USA), with $100 \mathrm{kN}$ load cell capacity. With the same plugs used in tension tests inserted in both ends, the specimens were mounted in the v-shaped grips and were grabbed with a pressure of 70 bar. An extensometer (Model 634.12F-24, MTS System Corporation, Eden Prairie, MN, USA) was placed at the center of the specimens to record the strain. Using the value obtained for the ultimate tensile strength (UTS) in tension tests, each specimen was subjected to a tension-tension cyclic loading starting at $30 \%$ UTS. The loading continued for 7000 cycles, which has been shown to be sufficient to produce temperature stabilisation [94, 154]. Specimens experienced a constant amplitude sinusoidal wave-form loading with a frequency of $5 \mathrm{~Hz}$ and a stress ratio of 0.1 . The maximum stress amplitude was then increase by 5\% UTS and the test specimen was subjected to another 7000 cycles. This procedure was repeated until the specimen failed.

An IRT camera (Silver 420, FLIR Systems Canada, Burlington, ON, Canada) was used to record the variations of temperature at each applied stress amplitude. The camera came with a spatial resolution of $32 \times 240$ pixels and a temperature resolution of $20 \mathrm{mK}$. The camera was synchronized 
to the controller of the mechanical testing machine in order to trigger image recording at the highest stress level in each loading step and to eliminate the effect of any temperature fluctuations due to thermoelastic heating $[94,154]$. Figure 7-3 illustrates the fatigue testing setup with the use of IR camera.

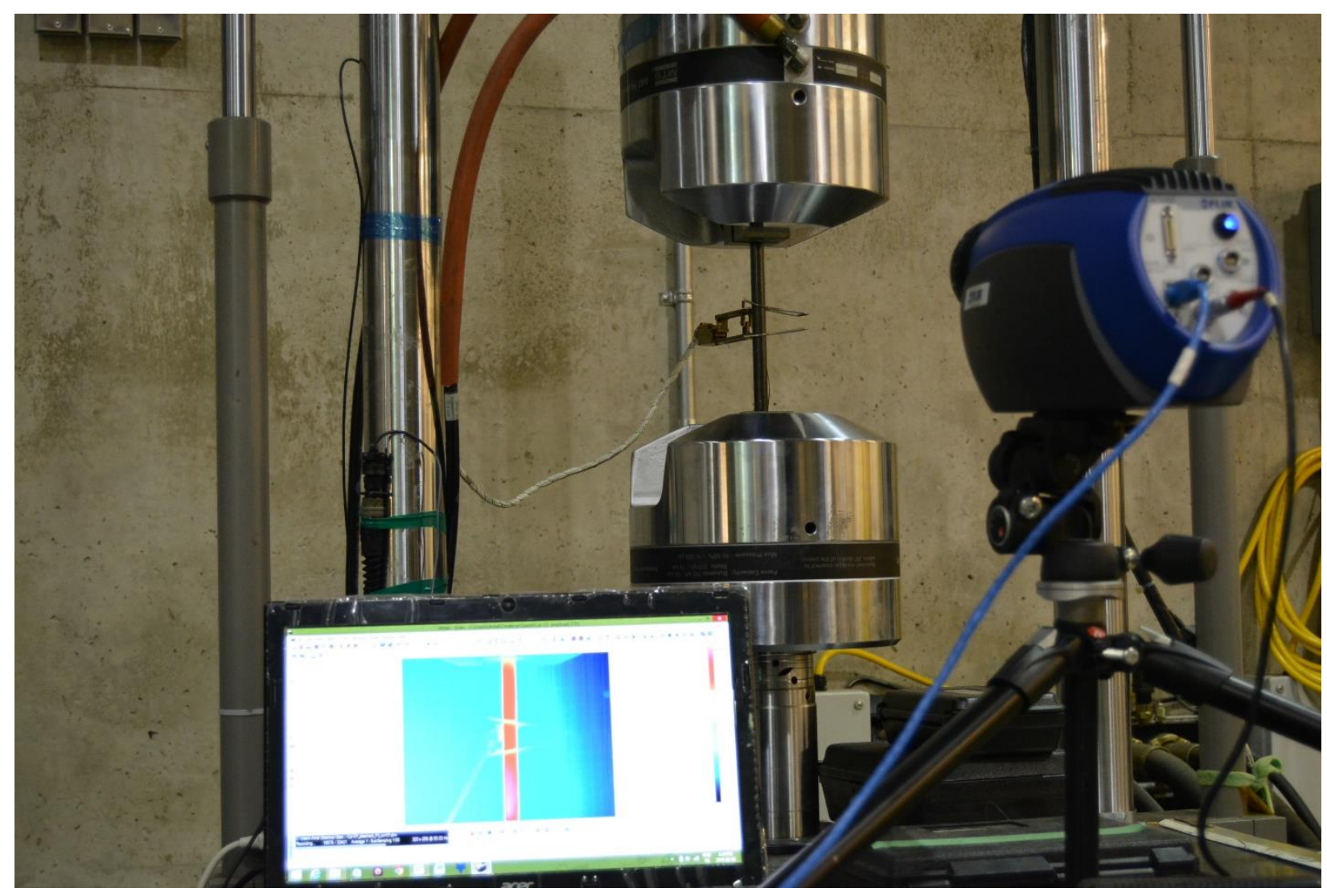

Figure 7-3. Experimental setup for fatigue testing using IR camera

\section{Measuring stiffness degradation}

To capture the evolution of damage (stiffness degradation), each specimen was quasi-statically loaded to the maximum stress at each level prior to applying the cyclic load. The data was then used to obtain the static damaged modulus $(E)$ (which will be referred to as static modulus) at each stress level. The schematic graph in Figure 7-4 shows how the elastic modulus was measured. The dynamic damaged modulus $\left(E^{*}\right)$ (which will be referred to as dynamic modulus) was also measured in each cycle from the linear portion of the stress-strain curve. 


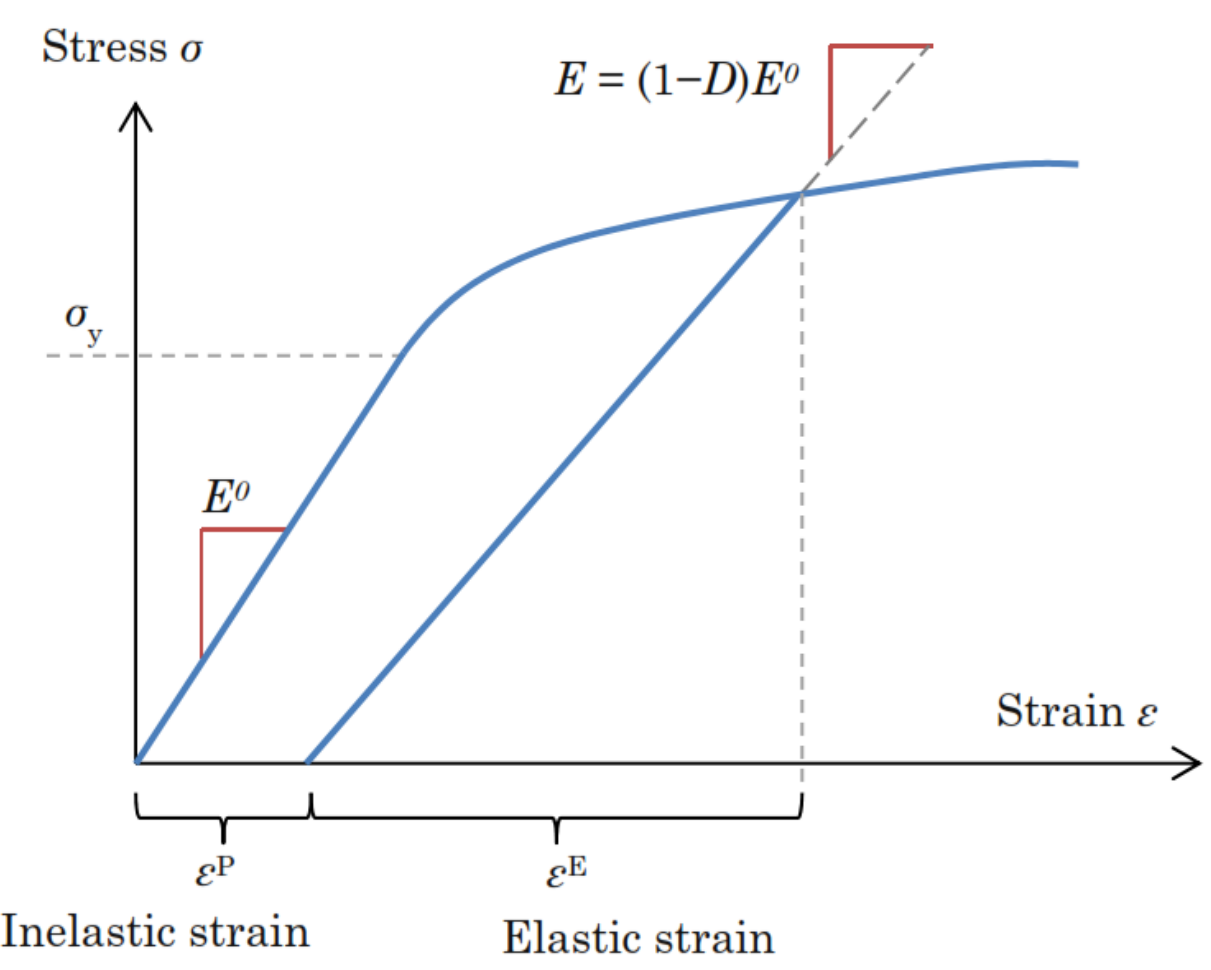

Figure 7-4. Schematic graph showing nonlinear response due to changing modulus and permanent inelastic strains [156].

\subsection{Results}

\subsubsection{Laminate microstructure}

The specimens' cross-sections were examined under an optical microscope to obtain the laminate porosity and the existing voids' sizes. The void fraction was found to be $2.49 \pm 1.44 \%$ and the largest void found on the examined surfaces was about $85 \mu \mathrm{m}$ wide.

\subsubsection{Moisture absorption results}

The results of the moisture absorption tests were shown in Figure 7-5. The measured weight gain of the composite tubes reached a steady state after about 35 days and was found to be $0.50 \pm 0.09 \%$ 


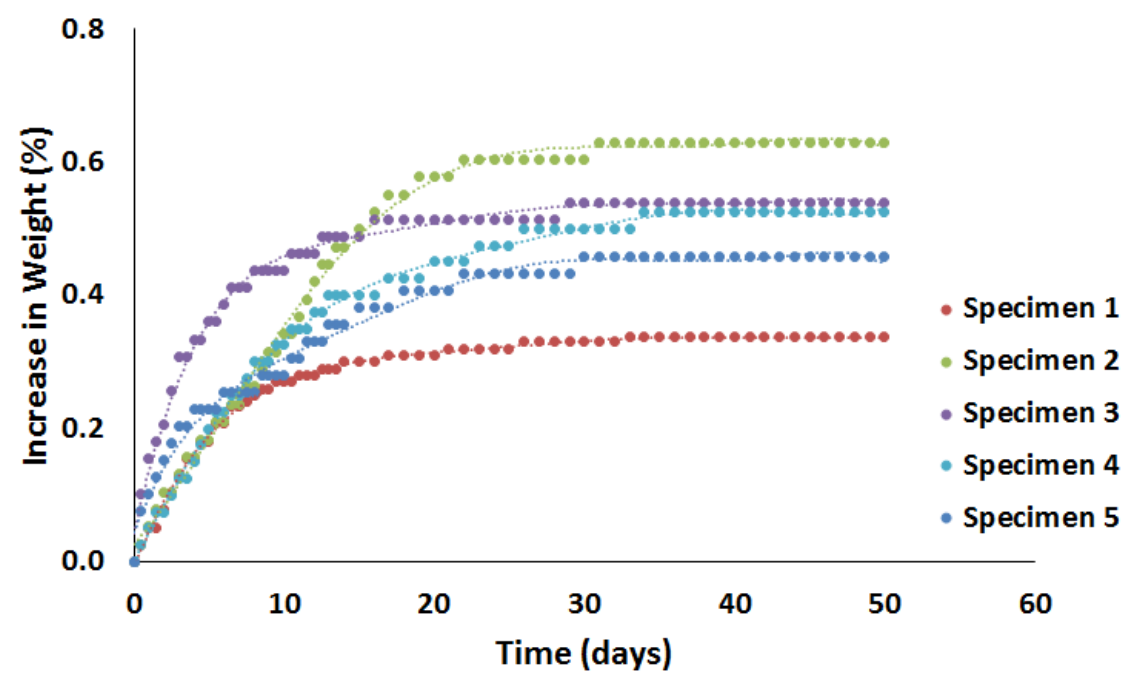

Figure 7-5. Moisture absorption results showing the weight gain of the composite tubes.

\subsubsection{Compression test results}

The load-displacement curves showing the behaviour of the composite tubes in compression are shown in Figure 7-6a. The compressive modulus and the compressive rigidity were found to be 10.0 $\pm 0.2 \mathrm{GPa}$ and $0.95 \pm 1.1 \mathrm{MN}$ respectively, and the ultimate compressive strength (UCS) was 316.9 $\pm 10.9 \mathrm{MPa}$. The maximum compressive load was found as 30.2 $\pm 1.1 \mathrm{kN}$. All tested specimens experienced a brittle failure in compression mainly due to local buckling of the outermost plies. Figure 7-6b illustrates a typical sample failed in compression.
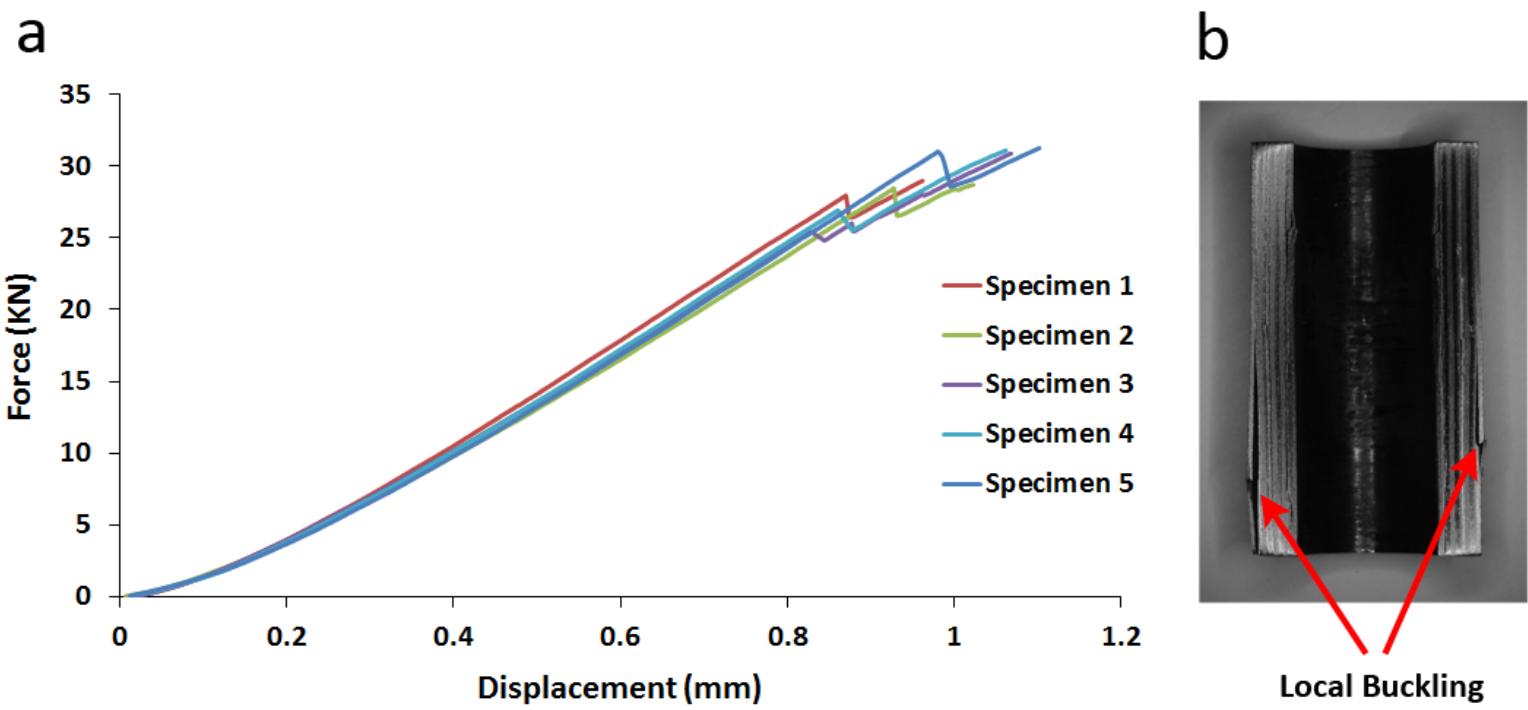

Figure 7-6. (a) Load-displacement diagram showing compression test results. (b) A typical specimen failed in compression and cut in half. The buckling of the outermost plies is evident. 


\subsubsection{Tension test results}

To capture both pre-failure and post-failure behavior of the composite specimens in tension, loaddisplacement diagrams were plotted (Figure 7-7). All the composite specimens showed linear elastic behaviour up to the point of failure where the two outermost layers (made of 0-direction fibres) ruptured and the whole load was transferred to the inner layers (Figure 7-7a). The specimens then experienced a few brittle fractures upon failure of the inner layers. The tensile modulus and the tensile rigidity were found to be $38.9 \pm 1.0 \mathrm{GPa}$ and $3.74 \pm 0.05 \mathrm{MN}$ respectively, and the ultimate tensile strength (UTS) was 403.9 $\pm 7.8 \mathrm{MPa}$. The maximum tensile load the specimens carried was $38.9 \pm 0.7 \mathrm{kN}$. Figure $7-7 \mathrm{~b}$ shows a typical specimen which failed in tension.

a

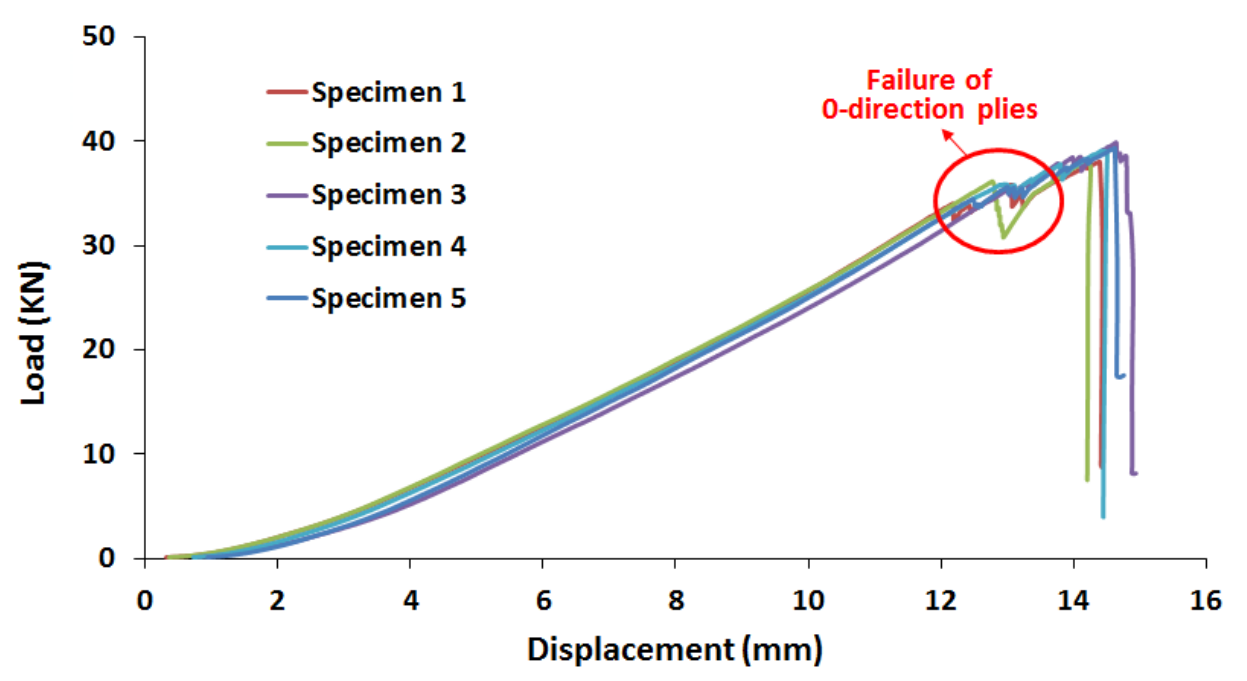

b

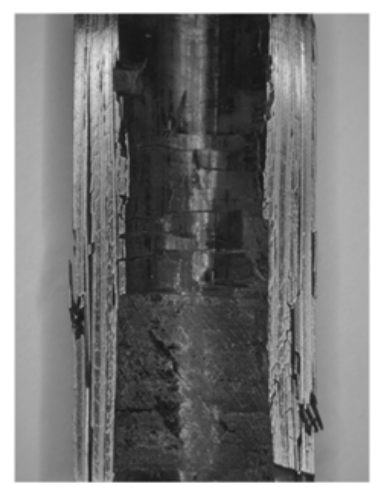

Figure 7-7. (a) Tension test results for the specimens. (b) A typical specimen failed in tension test.

\subsubsection{Bending test results}

The load-displacement diagram for three-point bending tests was shown in Figure 7-8a. The specimens showed linear behaviour prior to failure. Using Eq. (7.2), the flexural modulus of the specimens was calculated as $41.1 \pm 0.9 \mathrm{GPa}$. The bending rigidity (EI) was found to be $66.9 \pm 1.0$ $N . m^{2}$ and the ultimate flexural strength (UFS) was 405.3 $\pm 8.1 \mathrm{MPa}$. The failure started with rupture of 0 -direction fibres in the tension side and bucking of them in the compression side. 
Figure 7-8b illustrates a typical specimen that failed in bending and was cut parallel to the longitudinal axis to show the failure site.

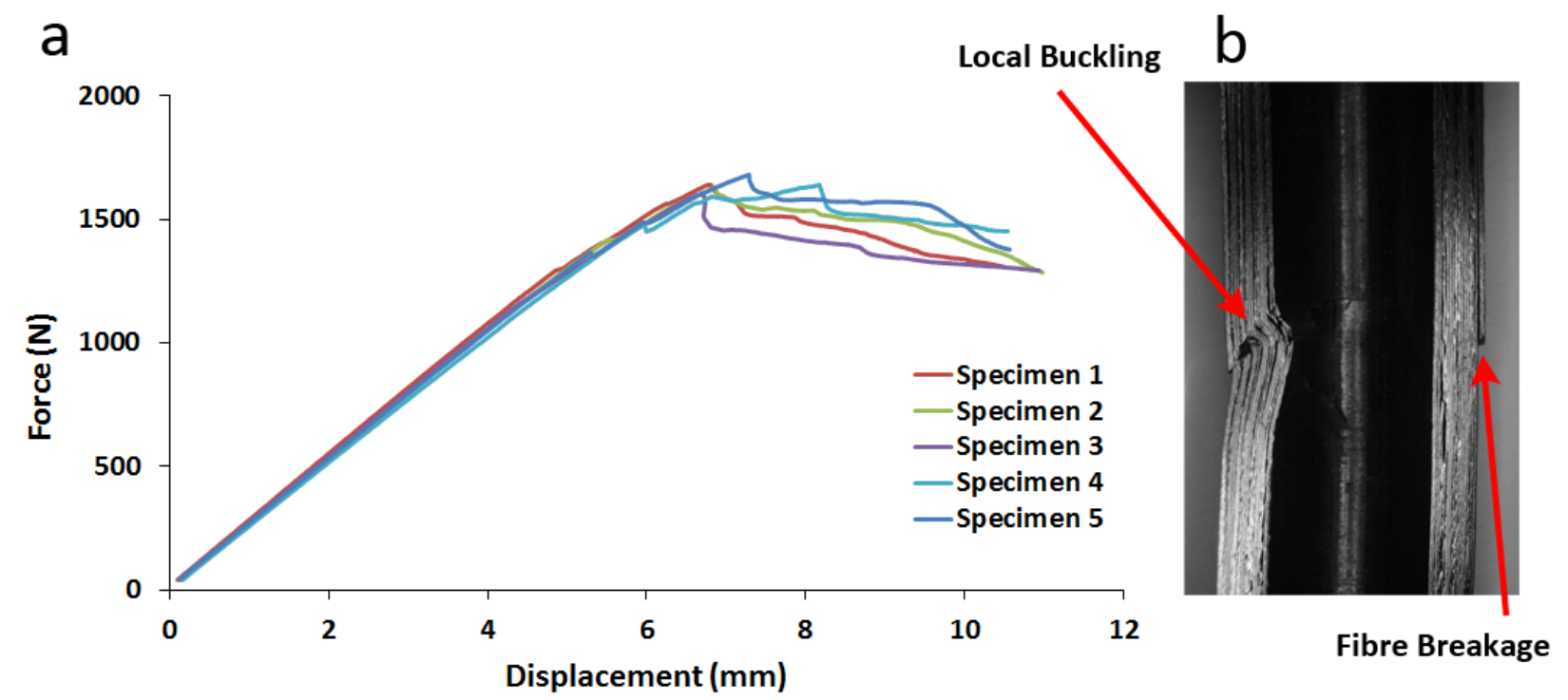

Figure 7-8. (a) 3-point bending test results for the specimens. (b) A typical specimen failed in the bending test. The specimen failed due to fibre breakage in the tension side and local buckling in the compression side.

\subsubsection{Elastic moduli comparison}

Statistical analysis revealed that there is a significant difference between the compressive and flexural moduli of the composite tubes $(\mathrm{P}<0.05)$. The average flexural modulus was $31.2 \mathrm{GPa}$ higher than the average compressive modulus of the tubes (95\% CI, 30.1 to $32.2 \mathrm{GPa}$ ).

A significant difference was also found between the tensile and compressive moduli of the composite tube $(\mathrm{P}<0.05)$. The average tensile modulus was 28.9 GPa higher than the average compressive modulus of the composite tubes (95\% CI, 27.7 to $30.1 \mathrm{GPa}$ ).

\subsubsection{Torsion test results}

Figure 7-9 shows the behaviour of the composite tubes in torsion by depicting twisting moment versus twist angle. The shear modulus and the torsional rigidity of the composite tubes were $22.4 \pm 0.5 \mathrm{GPa}$ and $70.7 \pm 2.0 \mathrm{~N} . \mathrm{m}^{2}$, respectively. The shear strength and the maximum twisting moment of the tubes were found to be $328.5 \pm 7.3 \mathrm{MPa}$ and 149.1 $\pm 6.3 \mathrm{~N} . \mathrm{m}$ respectively. All 
tested specimens showed linear elastic behaviors up the point of failure where delamination of outermost longitudinal plies and $45^{\circ}$-plies occurred. A typical failed specimen is shown in Figure 7-9, where the aforementioned delamination is visible.

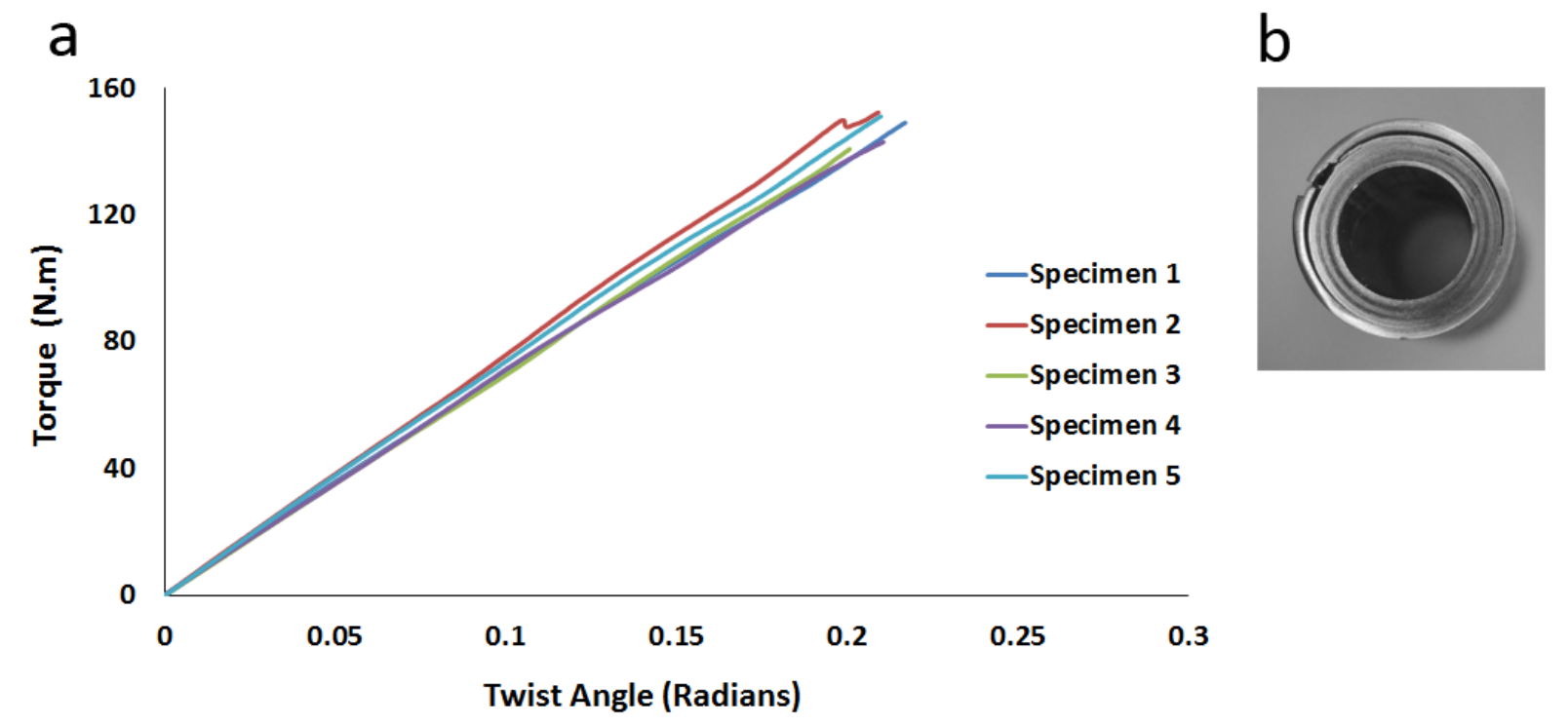

Figure 7-9. Torsion test results for the specimens and a typical specimen failed in the torsion test. Delamination between the 0 -direction plies and the adjacent plies is evident.

\subsubsection{Rockwell hardness results}

The average Rockwell B hardness value for the composite specimens was found to be $43.01 \pm 1.17$ HRB. All specimens showed circular-shaped indentations at the testing locations normal to the testing direction.

\subsubsection{Surface roughness results}

The average surface roughness of the specimens $\left(R_{a}\right)$ was found to be $0.636 \pm 0.077 \mu m$ and $0.387 \pm 0.042 \mu \mathrm{m}$ in the fibres direction and perpendicular to the fibres respectively. The surface texture in the fibres direction resulted in a higher roughness in the fibres direction. 


\subsubsection{Fatigue test results}

Surface temperature variation as a function of the number of loading cycles for a typical specimen was plotted in Figure 7-10, which was captured with the IR camera. Figure 7-11 shows a sequence of surface temperature, after stabilisation, for the same specimen. Up to a cyclic load of $65 \%$ UTS, the temperature contour was uniform across the specimen surface. As the load was increased, there was a region with a slightly higher temperature, where the specimen was eventually failed. This region is referred to as "hot zone" [154] and was marked in Figure 7-11. Using the stabilised temperature at each load level, the temperature increase was plotted against the maximum applied stress for the specimens (Figure 7-12). The plot depicts a bilinear behavior that results from the rapid change in the surface temperature rise. The fatigue limit was determined by finding the intersection of the two lines, which was found to be $70.3 \%$ UTS.

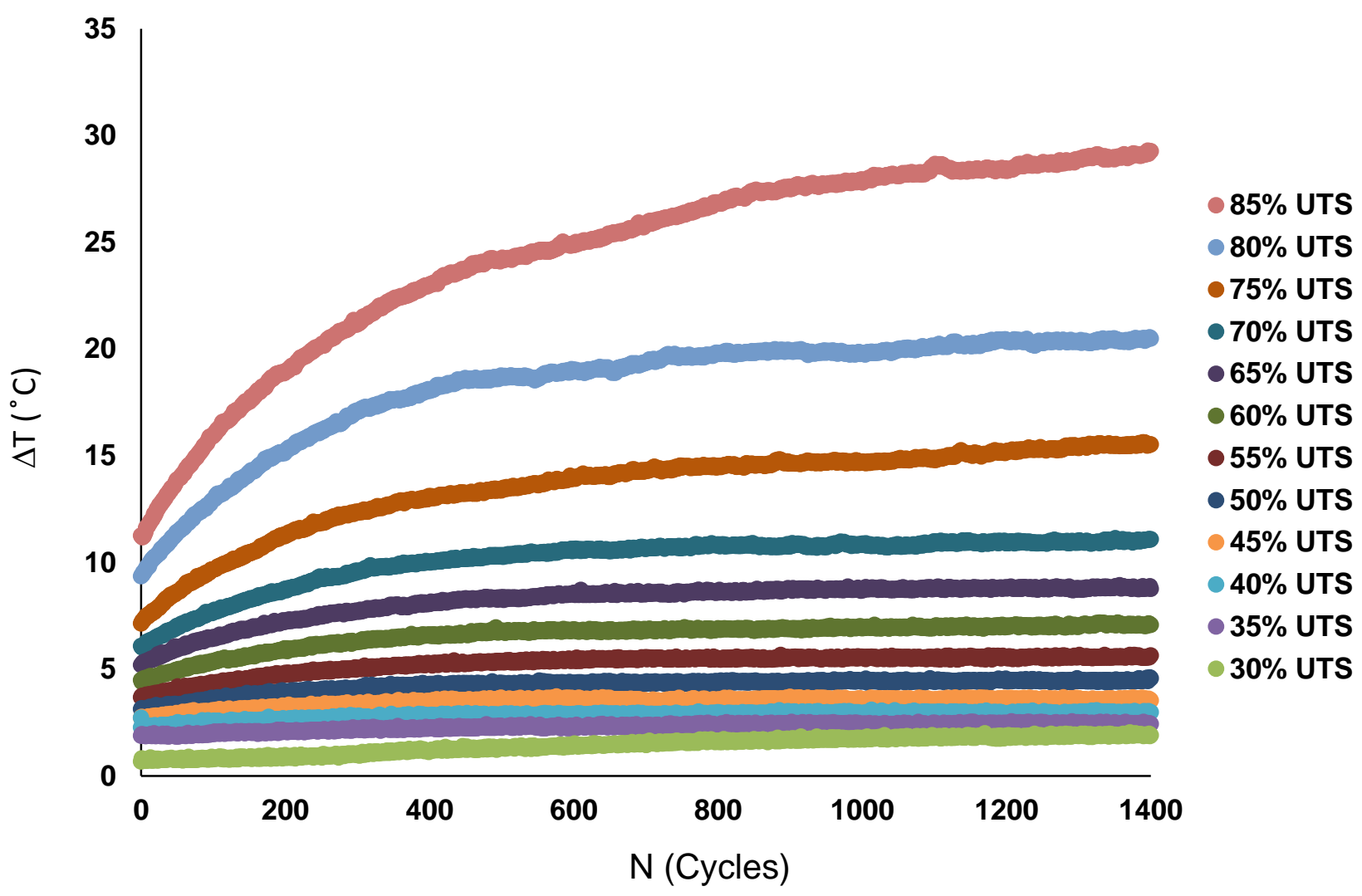

Figure 7-10. Variation of surface temperature versus number of cycles for different stress levels in a typical specimen 
The evolution of damage was investigated by obtaining the static and dynamic moduli as a function of the number of cycles. Figure 7-13 and Figure 7-14 show the static and dynamic moduli respectively versus the number of cycles for a typical composite specimen.

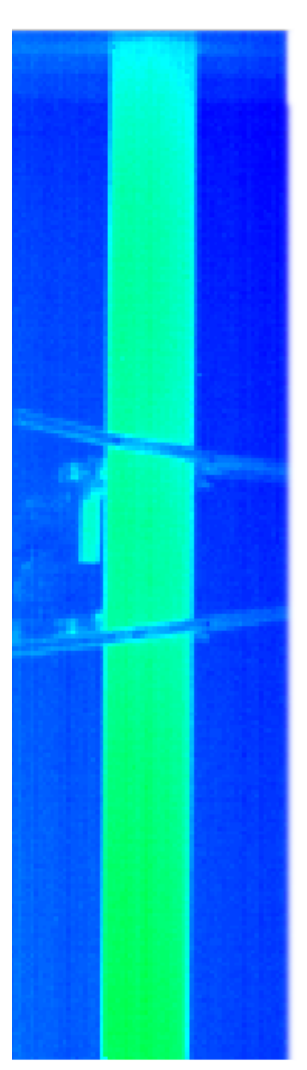

$60 \%$ UTS

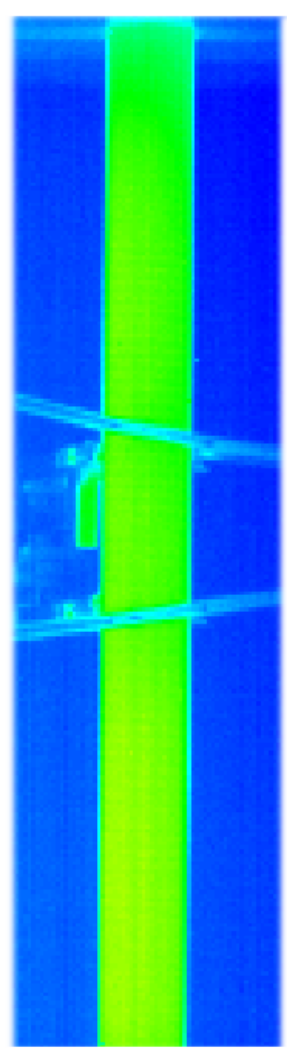

$70 \%$ UTS
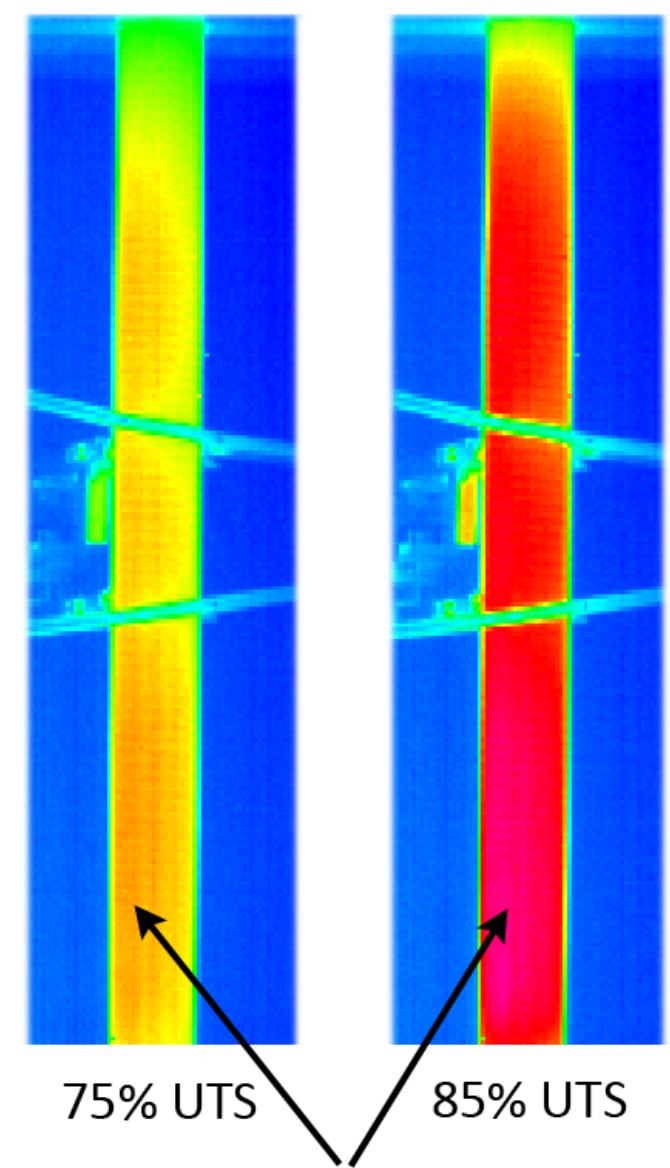

$\mathrm{T}\left({ }^{\circ} \mathrm{C}\right)$

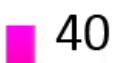

35

30

25

20

Figure 7-11. Thermographic temperature distribution contour following temperature stabilization at different stress level. UTS: ultimate tensile strength 


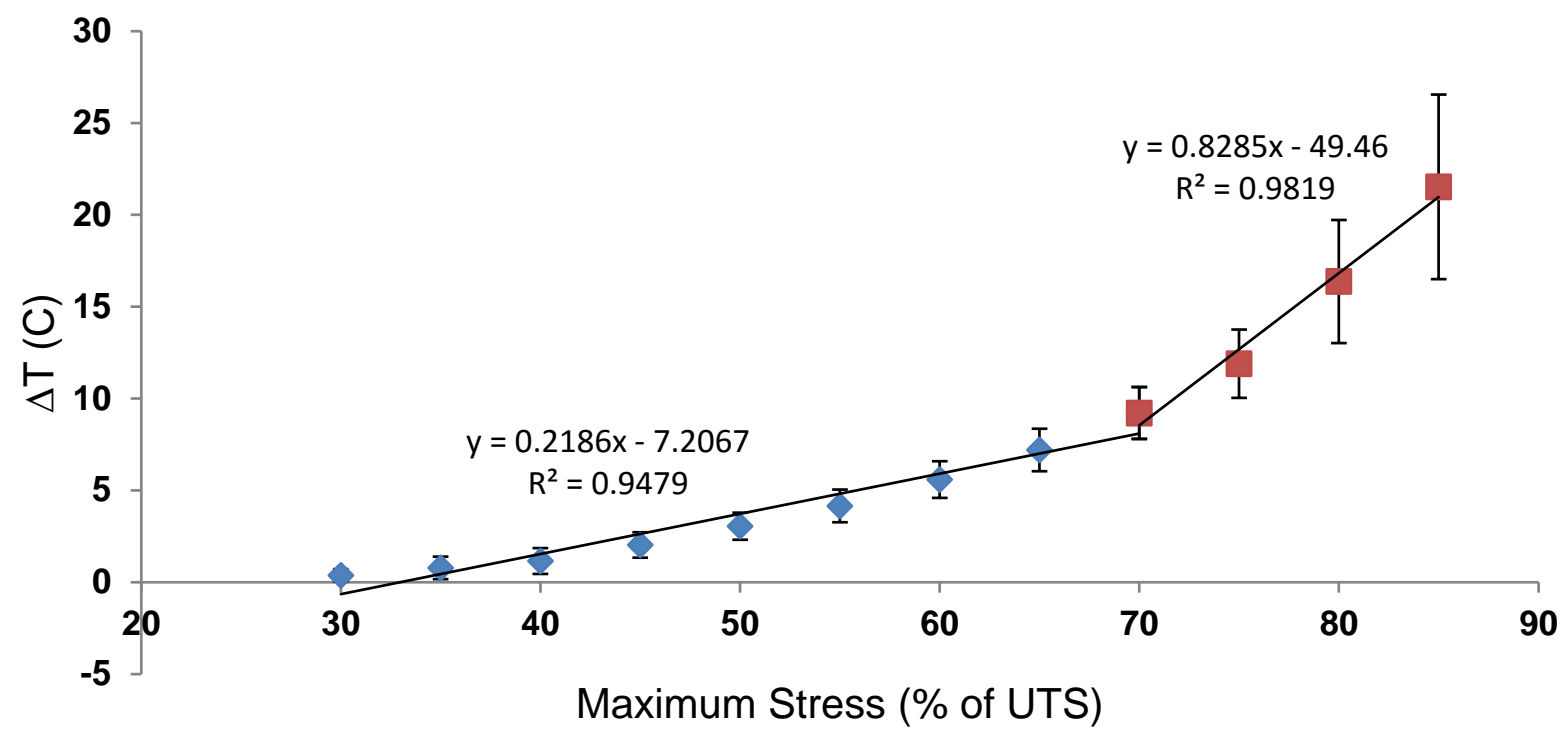

Figure 7-12. Thermographic results showing the variation of maximum stabilized temperature versus the maximum applied stress. The bilinear behavior of the data is evident in the figure.

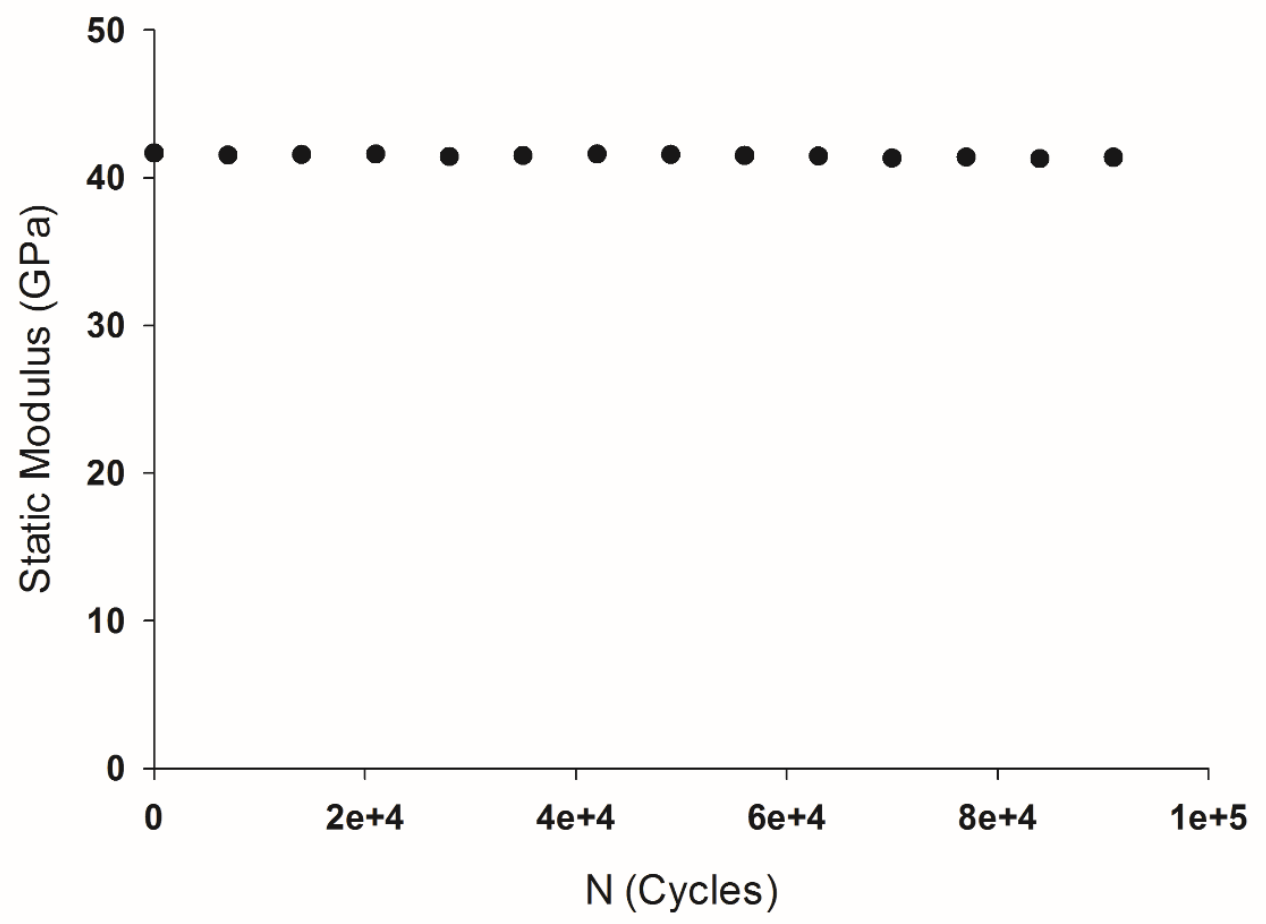

Figure 7-13. Static modulus versus number of cycles for a typical composite specimen 


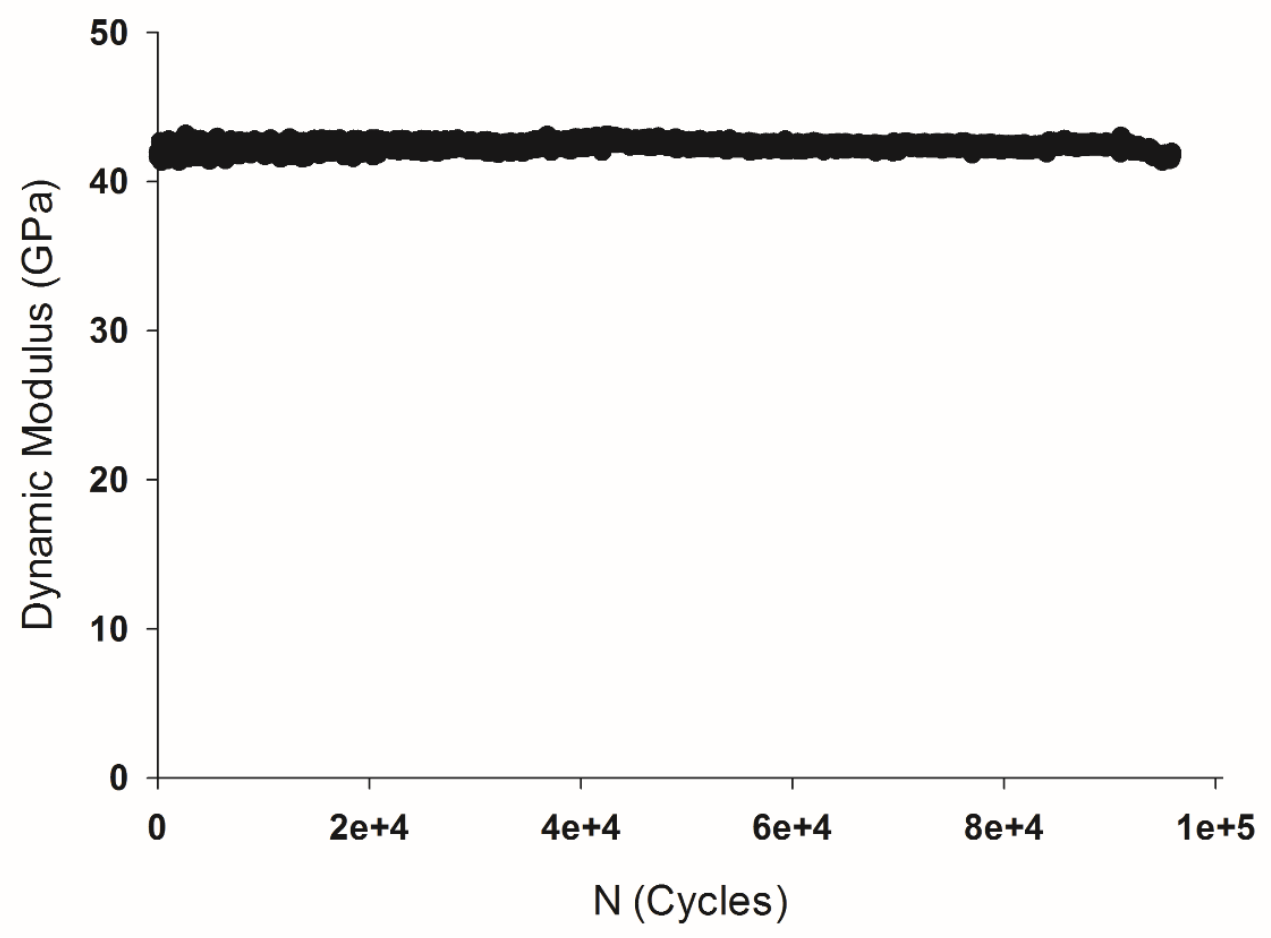

Figure 7-14. Dynamic modulus versus number of cycles for a typical composite specimen

\subsection{Discussion}

\subsubsection{General findings}

The proposed composite IM nail whose stacking sequence was optimized based on fracture healing requirements [146] was mechanically tested to obtain its stiffness, strength and modes of failure under different clinical types of load. Since an IM nail is subjected to various types of loading namely axial, flexural, and torsional following implantation, axial (tension and compression), bending, and torsion tests were carried out on the optimized composite IM nail structure to determine its mechanical properties. In addition, the failed specimens were cut normal to the tube axis and carefully examined to investigate the failure modes.

The test results revealed that the composite IM nail is strong enough for various types of physiologic loading that it is subjected to. Although the composite IM nail experienced a brittle fracture in almost all types of testing, the load levels that resulted in failure were extremely higher than what an IM nail would experience inside the human bone. For instance, the failure load in compression was $30.2 \pm 1.1 \mathrm{kN}$ which is more than ten times higher than the maximum 
compressive load applied on the nail in a bone-nail system assuming that the whole load is carried by the IM nail. In terms of stiffness, the composite IM nail is relatively rigid in bending and torsion while axially flexible. A conventional Ti-alloy IM nail has an axial rigidity of $6.7 M N$, a bending rigidity of $132.8 N . \mathrm{m}^{2}$ and a torsional rigidity of $98.9 \mathrm{~N} . \mathrm{m}^{2}$ [146]. The composite IM nail was $85 \%$ less rigid axially, while being only $49 \%$ and $28 \%$ more flexible in bending and torsion when compared to a conventional Ti-alloy IM nail. Comparing flexural and compressive moduli of the composite tubes revealed that the flexural modulus of the composite nail was more than 4 times its compressive modulus. This statistically significant difference $(P<0.05)$ proved the customizability of composite materials that could be used towards designing optimal structures for fracture fixation applications. Microscopic images of intact specimens showed a uniform laminate microstructure with a low porosity $(<3 \%)$. Moreover, the linear behaviour of the specimens prior to failure in different types of mechanical testing (Figure 7-6-7) revealed that no local failure (e.g. delamination of plies) occurred up until failure. Water absorption test results confirmed that the laminate does not absorb significant amount of moisture (weight gain $\sim 0.5 \%$ ) when placed in a humid environment making it desirable for in-vivo applications. The flexible yet strong structure of the composite nails that were customized based on fracture healing requirements has made it attractive for fracture fixation applications.

When subjected to cyclic loading, a composite structure can prematurely fail at load levels that are significantly lower that its ultimate strength, which can in turn limit its service life [157]. The CF/epoxy IM nail had a fatigue limit of $70.3 \%$ UTS. Considering the cross-sectional dimensions of the IM nail, such limit would require a load of approximately $27 \mathrm{kN}$ which is much more than clinical-type loads (normally 2.5-3 times body weight) to which a human long bone is subjected [113]. The HCFS of the current composite structure was about $283 \mathrm{MPa}$ which is comparable to 316L stainless steel materials that are clinically used (i.e. around $200 \mathrm{MPa}$ and $300 \mathrm{MPa}$ for cast and forged 316L stainless steel, respectively) [158].

The ribbed outer surface of the composite tubes had a smooth finish. The average surface roughness in the fibres direction (i.e. $0.636 \pm 0.077 \mu \mathrm{m}$ ) and perpendicular to the fibres (i.e. $0.387 \pm 0.042 \mu \mathrm{m}$ ) were comparable to that of Ti-6Al-4V (i.e. $0.320-0.870 \mu \mathrm{m}$ ) that are used in manufacturing conventional IM nails [159]. The adhesion and spreading ability of cells (fibroblasts) on implant surfaces affect tissue integration. Cell behaviour on biomaterial surfaces depends on cell-implant interactions. Surface texture, roughness, chemical composition strongly 
affect cellular responses in contact with the implants [160]. Cell attachment has a direct relationship with surface roughness [159, 160]. In a cementless hip implant for instance, osseointegration (bone ongrowth) is required for successful outcome of the hip replacement. Therefore, a surface roughness of 3-5 $\mu \mathrm{m}$ is generated by bombarding the titanium implant with small abrasive particles such as aluminum oxide (corundum) [161]. However, no bonding is required between the bone and the implant surface in an IM nail. In fact, secondary fracture healing is promoted at the presence of axial movement of fracture fragments. It is not unreasonable to expect a similar cell attachment rate between the composite IM nail and a conventional Ti-alloy nail due to their similar surface roughness. However, more investigations are required to study the actual cell behavior on the textured surface of the proposed composite nail.

The results of the fatigue tests also showed that the static and dynamic moduli of the specimens were almost constant versus the number of cycles and did not change compared to their initial values. The unique stacking sequence of the composite structure helped to maintain an almost constant level of stiffness up until failure. Changes in stiffness during cycling loading may indicate substantial fatigue damage, even though the polymer matrix composite may appear to be structurally intact overall [94]. Consequently, one of the major complications of using composite biomaterials is the gradual loss of their mechanical properties (i.e. stiffness) during cyclic loading caused by fatigue damage $[79,80]$. The constant stiffness of the proposed composite IM nail can therefore make it a candidate for use in treating long bone fracture since it indicates negligible fatigue damage in the structure prior to failure.

\subsubsection{Comparison with prior studies}

There are very few studies that considered the use of composite IM nails for diaphyseal fracture fixation. One recent study reported mechanical properties of an E-glass fibres in bending and torsion [95]. The flexural and shear moduli, and the ultimate flexural and shear strength found in the present work were compared to corresponding values reported by Moritz et al. [95] in Table 7-1. The current ultimate flexural strength was lower than that reported in their study. However, flexural and shear moduli obtained for the E-glass IM nail were significantly lower than the current values. In particular, the reported shear modulus is $8.5 \%$ of that obtained for the current CF/epoxy IM nail and only $4.3 \%$ of that of Ti-alloy IM nail. This low shear modulus of the IM nail which results in a low torsional stiffness raises concern that the nail may cause fracture 
instability once it is used to fix human diaphyseal fractures. In addition, the shear strength reported in the mentioned study is $18 \%$ of that of a Ti-alloy nail, while the shear strength of the current CF/epoxy nail was found to be $60 \%$ of that in a Ti-alloy IM nail suggesting that the proposed $\mathrm{CF} / \mathrm{epoxy}$ nail is superior in fracture stabilisation.

Table 7-1. Mechanical properties of Ti-alloy, the current CF/epoxy and some previously developed composite IM nails. $E_{f}$ and $G$ are elastic and shear moduli, and $\sigma_{U}{ }^{f}$ and $\tau_{U}$ are ultimate flexural and shear strength respectively.

\begin{tabular}{c|c|c|c|c|c} 
Materials & \multicolumn{1}{c}{$\boldsymbol{E}_{f(\mathbf{G P a})}$} & $\mathbf{G}(\mathrm{GPa})$ & $\sigma_{U}^{f}(\mathbf{M P a})$ & $\tau_{U}(\mathbf{M P a})$ & Reference \\
\hline $\begin{array}{c}\text { Ti-alloy } \\
\text { Current optimized } \\
\text { CF/epoxy }\end{array}$ & 113.8 & 44 & 950 & 550 & - \\
\hline $\begin{array}{c}\text { UD E-glass/ } \\
\text { TEGDMA/ }\end{array}$ & $17.1 \pm 0.9$ & $22.4 \pm 0.5$ & $405.3 \pm 8.1$ & $328.5 \pm 7.3$ & - \\
\hline BisGMA & $0.5 \pm 0.1$ & $802.0 \pm 112.7$ & $39.2 \pm 5.0$ & $\begin{array}{c}\text { Moritz et al. } \\
{[95]}\end{array}$ \\
\hline $\begin{array}{c}\text { UD-Braided E-glass/ } \\
\text { TEGDMA/ BisGMA }\end{array}$ & $15.3 \pm 1.8$ & $1.9 \pm 0.6$ & $602.0 \pm 81.6$ & $99.5 \pm 14.7$ & $\begin{array}{c}\text { Moritz et al. } \\
{[95]}\end{array}$ \\
\hline
\end{tabular}

\subsubsection{Limitations}

This chapter has few limitations. First, all the tests were performed under quasi-static loading conditions to find the basic mechanical properties of the composite nails. Cyclic loading of various types are required to determine fatigue resistance of the proposed composite nail. Second, the composite IM nail was manufactured straight, while an actual IM nail has a radius of curvature ranging from 1.5 to $3 \mathrm{~m}$ in the sagittal plane [162]. While all the ASTM tests performed required straight rather than curved specimens, the ignorance of the curvature can slightly affect the rigidity and the load-to-failure of the composite tubes. For example, the compressive load-to-failure can 
be reduced as a result of specimen's curvature since this would make the tube prone to buckling. Therefore, further mechanical testing is required on curved composite tubes to determine their mechanical performance.

In this thesis, Rockwell hardness test was chosen instead of Shore (durameter) hardness test which is another method for measuring hardness of plastics, because it is more generally used for harder plastics such as nylon, epoxy, and polycarbonate [81]. Rockwell hardness tests were performed to compare the hardness of the proposed composite specimens to that of conventional Ti-alloys used for manufacturing IM nails. It should be noted that there is no known relationship between Rockwell hardness values and the strength of fibre-reinforced composites and the obtained hardness values may not be used to measure the strength of the composite specimens.

The relatively large coefficient of variation (18\%) in the water absorption rate could be due to the manufacturing process (i.e. roll wrapping) which resulted in a variation in the micro-structure of the laminate. The relatively large coefficient of variation in the void percent (57.8\%) can be a result of this variation as well. However, such variation at the micro-structure level did not seem to significantly affect the mechanical properties of the composite specimens as confirmed by the results of tension, compression, bending, and torsion tests. The variation in the micro-structure can be minimized by choosing a more advanced manufacturing processes such as advanced fibre placement (AFP). This may slightly add up to the cost of the product but will result in a higher quality and more uniformness in the structure of the composite nails.

Fatigue tests were performed at a frequency of $5 \mathrm{~Hz}$ which was above the range of walking frequency (i.e. $3-5 \mathrm{~Hz}[163,164])$, since this frequency is the minimum required working frequency for the IR camera. Any frequency lower than $5 \mathrm{~Hz}$ can violate the adiabatic condition and result in poor quality images. Moreover, the cyclic loads were applied as a constant sinusoidal wave which does not fully simulate the hip joint force in the gait cycle. This hip joint force has two peak values in the stance phase of each cycle and does not have a pure sinusoidal pattern. Separate fatigue tests with a loading regime similar the hip joint force during the gait cycle must be performed on the specimens to confirm the obtained fatigue limit for this application. Further, specimens were tested at room temperature. Higher temperatures (e.g. body temperature) may have an effect on the polymer matrix properties as the matrix viscoelastic response characteristics are temperature-dependant. This is especially important when the specimen is subjected to cyclic 
loading. Therefore, fatigue tests at higher temperatures must be performed on the specimens to obtain their fatigue limit in elevated temperatures.

Finally, the current work is a biomechanical study aiming to obtain mechanical properties and to assess the modes of failure of the proposed composite IM nail whose structure was customized based on fracture healing requirements in chapter 6 of this thesis. The in-vivo performance of the proposed composite IM nail must be evaluated using animal studies and ultimately clinical trials before it can be used as an alternative to conventional metallic IM nails.

\subsubsection{Clinical implications}

Several studies reported reduction in bone mineral density due to stress shielding around IM nails [60, 139, 165]. Allen et al. [139] reported a significant overall bone mineral density decrease in healed tibiae with retained IM nails in a clinical study. Sha et al. [60] showed in an animal study that a low-rigidity nail manufactured from a titanium alloy (Ti-24Nb-4Zr-7.9Sn) exhibited better external callus formation, and reduced the effects of stress shielding and bone resorption when compared to a more rigid nail. They also showed that the low-rigidity nail was sufficiently strong enough to maintain alignment of the fracture in the osteoporotic rat model without delaying union.

Stress shielding phenomenon is not necessarily disadvantageous and can be beneficial in fracture healing. If the implant adequately counters bending, torsional, and shear stresses while only fractionally resisting compressive stress, it can thereby stimulate remodelling of the callus at the fracture site [85]. This concept is referred to as "selective stress shielding" [48], and is gaining attention in the design of fixation implants as the new generation of materials become available [135]. The current composite IM nail is significantly more flexible axially compared to the conventional metallic nails, while having comparable bending and torsional rigidities to those of a Ti-alloy IM nail. Reducing the axial rigidity without directly reducing the bending and torsional rigidity is technically impractical using conventional metallic alloys as axial, bending, and torsional rigidity directly depend on the nail geometry only for an isotropic material.

Conventional IM nails made of Ti-alloy absorb between $70-74 \%$ of the total axial force during the stance phase and $91 \%$ during the swing phase of gait cycle [5], shielding the fracture from compressive loads and resulting in bone resorption in post healing stages. The proposed composite IM nail with its unique structure which has been customized to yield the best mechanical 
performance as a fracture fixation device (i.e. to immobilize fracture fragments while allowing for compressive stress) can allow more load sharing compared to Ti-based nails.

Mechanical testing simulating the different types of loading the implant would experience revealed that the implant is strong enough to carry clinical-type loads. Successful use of fracture fixation devices made of carbon fibres in a number of animal studies and clinical trials confirm the biocompatibility of this material $[64,79]$.

Based on the current mechanical testing results of the previously optimized IM nail, the proposed composite nail has the potential to be used as an alternative to conventional Ti-based IM nails.

\subsection{Summary}

The purpose of this chapter was to evaluate the mechanical properties of a previously developed composite IM nail made of carbon-fibre/epoxy whose structure was optimized based on fracture healing requirements. Following manufacturing, the cross-section of the composite nail was examined under an optical microscope to find the porosity of the structure. Mechanical properties of the proposed composite intramedullary nail were determined using standard tension, compression, bending, and torsion tests. The failed specimens were then examined to obtain the modes of failure. The material showed high strength in tension (403.9 \pm 7.8 $M P a$ ), compression (316.9 $\pm 10.9 \mathrm{MPa})$, bending $(405.3 \pm 8.1 \mathrm{MPa})$, and torsion $(328.5 \pm 7.3 \mathrm{MPa})$. Comparing the flexural modulus $(41.1 \pm 0.9 \mathrm{GPa})$ with the compressive modulus $(10.0 \pm 0.2 \mathrm{GPa}$ ) yielded that the material was significantly more flexible in compression than in bending. This customized flexibility along with the high torsional rigidity of the nail $\left(70.7 \pm 2.0 \mathrm{~N} . \mathrm{m}^{2}\right)$ have made it ideal as a fracture fixation device since this unique structure can stabilize the fracture while allowing for compression of fracture ends. Negligible moisture absorption $(\sim 0.5 \%)$ and low porosity of the laminate structure $(<3 \%)$ are other power points of the proposed structure. The composite IM nail showed a fatigue limit of $70.3 \%$ of its ultimate tensile strength $(\sim 27 \mathrm{kN})$. Moreover, the rigidity of the structure was relatively constant up to the failure. The findings suggested that the carbonfibre/epoxy IM nail is flexible axially while being relatively rigid in bending and torsion and is strong enough in all types of physiologic loading; making it a potential candidate for use as an alternative to the conventional titanium-alloy intramedullary nails. 


\section{CONCLUSIONS}

Intramedullary (IM) nails are used as internal fracture fixation tools to treat diaphyseal fractures in long bones. They are implanted within the IM canal and are fixed by interlocked screws to stabilise the bone fragments during the healing process. Despite all the advancements in IM nail design used for treating femoral diaphyseal fractures, complications remain such as failure of the nail or screws, delayed union, non-union, and bone re-fracture [4]. In virtually all femoral shaft fractures, the definitive treatment protocol is to implant an antegrade, reamed, locked IM nail [32]. Such placement of nails can facilitate transfer of the load and stress through both the fractured femur and/or the nail itself. Titanium (Ti)-alloys commonly used in manufacturing IM nails, provide appropriate stability at the fracture site. However, their high rigidity causes the nail to bear the majority of the load once implanted, shielding the bone from the stress it would naturally experience [5]. The reduction of the mechanical stress on the femur results in bone resorption at the vicinity of the implant over time which is referred to as stress shielding [6]. This condition makes the bone prone to re-fracture. Low rigidity fracture fixation devices can favourably increase the compressive normal force at the fracture site, which in turn can boost fracture healing [85]. However, a fixation that is very flexible can lead to fracture instability with subsequent complications such as delayed- or mal-union [9, 82]. An ideal fixation implant must resist the shear movement of fracture ends while allowing for some level of compression at the fracture site. Designing an IM nail with such capabilities is technically impractical with conventional metallic alloys, since bending, and torsional rigidities directly depend on the nail geometry for an isotropic material. Fibre-reinforced composites have been used as an alternative material of choice in the design of internal fracture fixation implants, primarily bone fracture plates because of their high strength, low rigidity, and corrosion and fatigue resistance. However, there are very few studies that have considered composite materials for IM nail design. Moreover, to the author's knowledge, no study in the literature has considered the optimization of such composite structures to fulfil the requirement of fracture healing.

The main objective of this thesis was to develop a fibre-reinforced composite IM nail made of $\mathrm{CF} /$ ppoxy for treatment of femoral shaft fractures. Since the aim of the optimization process was to lower the axial rigidity of the IM nail while keeping it relatively rigid in bending and torsion, the process started by obtaining closed-form expressions for axial, bending, and torsional rigidities 
of a composite tube. The derivation of the rigidity formulas was done in chapter 4 using the combination of classical laminate theory and beam theory. The obtained formulas were validated against the rigidity values obtained through 3-dimensional (3D) finite element models which took into account the out-of-plane response of the composite laminate as well as the interaction amongst the layers (e.g. normal and shear interlaminar stresses). One crucial factor that must be considered when designing a composite structure is the presence of structural couplings. These couplings can lead to undesirable deformations and may interfere with the component functionality. The conditions (e.g. particular stacking sequences of the laminate) that result in such couplings were discussed in chapter 5 . In particular, a coupling factor $(\lambda)$ was introduced in this chapter that detects extension-twist coupling in composite tubes.

The optimization process was performed in chapter 6 in two steps. In the first step, the expressions derived in chapter 4 were utilized to optimize the composite nail in order to obtain the lowest axial rigidity with the highest bending and torsional rigidities by altering the stacking sequence and the thickness of the laminate. The top seven candidates grouped based on their bending rigidity, were then examined in an experimentally validated finite element model to evaluate the performance of the IM nail candidates in realistic boundary conditions. In this regard, the finite element model of bone-nail assembly was developed and major muscle loads were applied on the femur. Two stages were simulated, i.e., post-operative and healed bone stages. In the first stage, the composite IM nail was assessed in the presence of transverse and oblique shaft fractures, whereas in the second stage, in which bone continuity was assumed to be recovered, the long-term response of the bone to the composite implant was of interest and thus the bone loss due to the presence of the composite implant was compared to that with conventional Ti-alloy IM nails.

It was found that a composite nail with an outer diameter of $14 \mathrm{~mm}$, an overall thickness of 2.65 mm, 17 layers of carbon fibre (CF)/epoxy made of $24 \mathrm{~K}$ standard modulus carbon fibres, and a stacking sequence of $\left[0_{2} /-45 / 45_{2} /-45 / 0 /-45 / 45_{2} /-45_{2} / 45_{2} /-45 / 90_{2}\right]$ with the 0 -direction plies as the outermost plies, showed an overall superiority compared to the other configurations. The configuration showed an axial rigidity of 3.7 $M N$, and bending and torsional rigidities of 70.3 and $70.9 N . m^{2}$ respectively. It increased compression at the fracture site by $344.9 \mathrm{~N}$ on average, while maintaining fracture stability through an average increase of only $0.6 \mathrm{~mm}$ in fracture shear movement in transverse and oblique fractures when compared to a conventional Ti-alloy nail. The 
long-term results suggested that the proposed composite IM nail reduces bone loss in the femoral shaft from $7.9 \%$ to $3.5 \%$ when compared to a conventional Ti-alloy nail.

In the next stage of the thesis, the optimized structure was manufactured by roll-wrapping the prepreg sheets around a mandrill and then spiral wrapping the material for compression. Mechanical characterization of the circular tubes was performed through standard quasi-static and fatigue testing. As predicted by the developed closed form expressions in chapter 6 , the optimized structure was essentially more rigid in bending that in tension and compression. The average bending modulus was $31.2 \mathrm{GPa}$ higher than the average compressive modulus of the tubes $(95 \%$ CI, 30.1 to $32.2 \mathrm{GPa}$ ). Further, the values for axial, bending, and torsional rigidities of the composite tubes (i.e., 3.74 $\pm 0.05 \mathrm{MN}, 66.9 \pm 1.0 \mathrm{~N} . \mathrm{m}^{2}, 70.7 \pm 2.0 \mathrm{~N} . \mathrm{m}^{2}$ respectively) obtained through mechanical testing agreed well with those obtained from analytical formulas (i.e., 3.70$M N, 70.3 N . m^{2}, 70.9 N . m^{2}$ respectively), indicating the validity of the assumptions in the analytical formulation in chapter 6 . The optimized nail showed high strength in tension $(403.9 \pm 7.8 \mathrm{MPa})$,

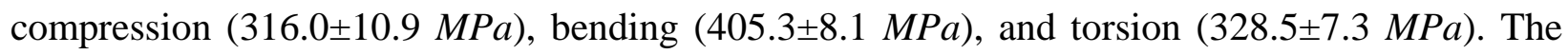
load levels that resulted in failure of the composite specimens were extremely higher than what an IM nail would experience inside the human bone. For instance, the failure load in compression was 30.2 $\pm 1.1 \mathrm{kN}$ which is more than ten times higher than the maximum compressive load applied on the nail in a bone-nail system assuming that the whole load is carried by the IM nail. Negligible moisture absorption $(\sim 0.5 \%)$ and low porosity of the laminate structure $(<3 \%)$ were other power points of the proposed structure. The proposed composite IM nail showed a fatigue limit of $70.3 \%$ UTS (ultimate tensile strength). Considering the cross-sectional dimensions of nail, such limit would require a load of approximately $27 \mathrm{kN}$ which is much more than clinical-type loads (normally 2.5-3 times body weight) to which a human long bone is subjected. Moreover, the high cycle fatigue limit of the proposed structure was about $283 \mathrm{MPa}$ which is comparable to $316 \mathrm{~L}$ stainless steel materials that are clinically used (i.e. around $200 \mathrm{MPa}$ and $300 \mathrm{MPa}$ for cast and forged 316L stainless steel, respectively). The structure also showed relatively constant static and dynamic moduli with the application of cyclic loading. The unique stacking sequence of the composite structure helped to maintain an almost constant level of stiffness up until failure. The constant stiffness of the proposed composite IM nail can therefore make it a candidate for use in treating long bone fracture since it indicates negligible fatigue damage in the structure prior to 
failure. Overall, the findings of this thesis suggest that the CF/epoxy IM nail is a potential candidate for use as an alternative to the conventional Ti-alloy IM nails.

\subsection{Contributions}

- Employed closed-form expressions for structural rigidities of a composite plate derived from the classical laminate theory, along with the finite element (FE) method to design a composite bone fracture plate (made of carbon fibre/epoxy) customized to fulfill the mechanical requirements of bone fracture healing.

- Employed validated closed-form expressions for structural rigidities of circular composite tubes as a function of their geometry, material properties, and stacking sequence of the laminate for optimization of composite IM nails.

- Introduced a factor for detecting extension-twist coupling in circular composite tubes with circumferentially uniform stiffness (CUS) configuration.

- Optimized a composite IM nail using the obtained closed-form expressions and a comprehensive FE model based on the selective stress shielding approach (i.e. to make the fixation device rigid in bending and torsion while making in axially flexible).

- Related significant factors involved in fracture healing to mechanical parameters measurable in finite element analysis.

- Proposed guidelines for design of composite IM nails for treating femoral shaft fractures.

- Proposed a composite IM nail which is sufficiently strong to withstand all clinical-type loading (based on mechanical testing results).

- Proposed a composite IM nail whose stiffness stays constant until failure when subjected to cyclic loading.

\subsection{Future work}

The manufactured prototypes did not have any curvature along the long axis of the nail as to make them capable of being tested using standard testing setup. The actual IM nail normally has a radius of curvature of approximately $1.5 \mathrm{~m}$ to mimic the curvature of the human femur. Therefore, manufacturing the curved composite IM nail and using it in synthetic femurs with shaft fractures 
could be the topic of a new biomechanical study. The results of such biomechanical testing can be compared to those from a similar testing setup in which the fracture is fixed with a conventional Ti-alloy IM nail.

A different type of fatigue testing with the presence of holes and locking screws can be performed to investigate the response of the composite IM nail in the vicinity of the holes to cyclic loading. Different designs of bushing inside the screw holes could be considered in case the composite structure needs to be strengthen in those areas.

Although the composite nail has a comparable average surface roughness to that of a conventional Ti-alloy nail, the ribbed surface of the composite nail can result in a different cell response. Moreover, from an orthopaedic surgeon's point of view, the surface texture of the composite nail resulting from the manufacturing process can make insertion and removal of the device difficult. Thus, the possibility of a biocompatible coating on the nail surface which has comparable cell response to that of $\mathrm{Ti}-6 \mathrm{Al}-4 \mathrm{~V}$ can be considered.

The use of medical grade epoxy and its possible effect of the material properties of the composite IM nail needs to be investigated. Carbon-fibre implants have an established history of clinical use with excellent biocompatibility and no cytotoxicity. However, separate biocompatibility tests need to be performed on the proposed structure to evaluate its effect on the osteogenesis (i.e. new bone formation by osteoblast cells) process in comparison with a medical grade metal material obtained from a commercially-used clinical IM nail. Finally, the performance of the proposed IM nail must be evaluated in-vivo. Thus, animal studies and ultimately clinical trials are future phases of this project.

As for the animal study, since the dimensions, number of layers, and stacking sequence of the IM nail have been optimized for treating shaft fractures in the human femur, it cannot be used in small animals such as rats. Briefly, a series of dogs could be randomly assigned into two groups. Following creating mid-shaft femoral fractures in the right femur of all animals in the study, the fractures in one group would be fixed with the composite nail, while fractures in the other group would be repaired with a conventional IM nail. The contralateral femur in each group would serve as control. The same number of dogs from both groups would be sacrificed at different time points for a period of about 2 years, and the implanted and control femurs would be harvested and mechanically tested in torsion. The quality and the amount of callus formed and the corticalization 
of the regenerated bone can be assessed by comparing the rigidity and maximum torque in both groups. 


\section{APPENDICES}

\section{A 1. MATLAB code used to calculate the structural rigidities of composite bone plates}

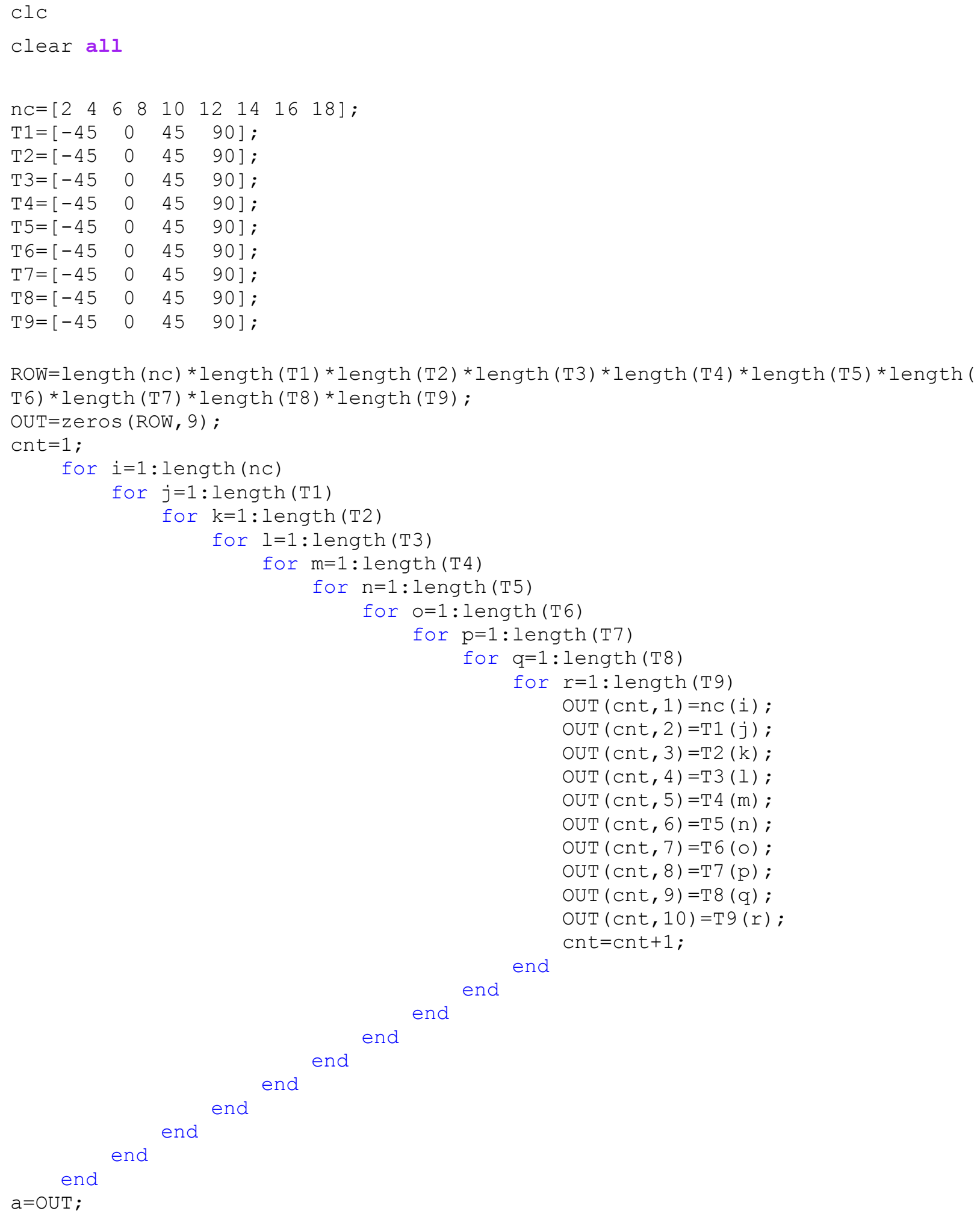




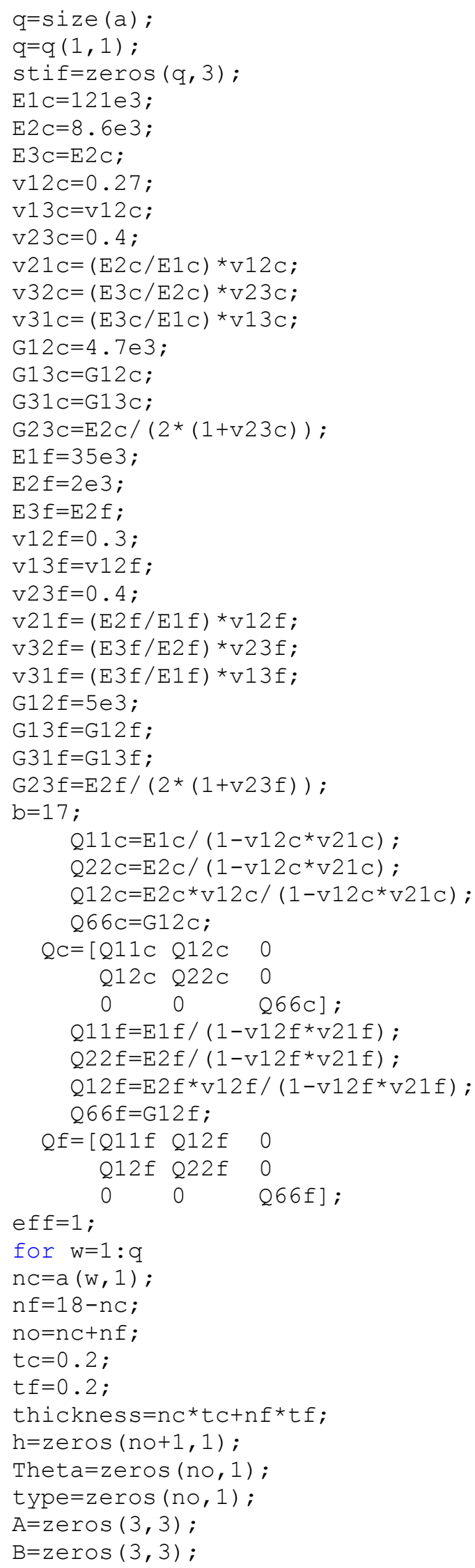




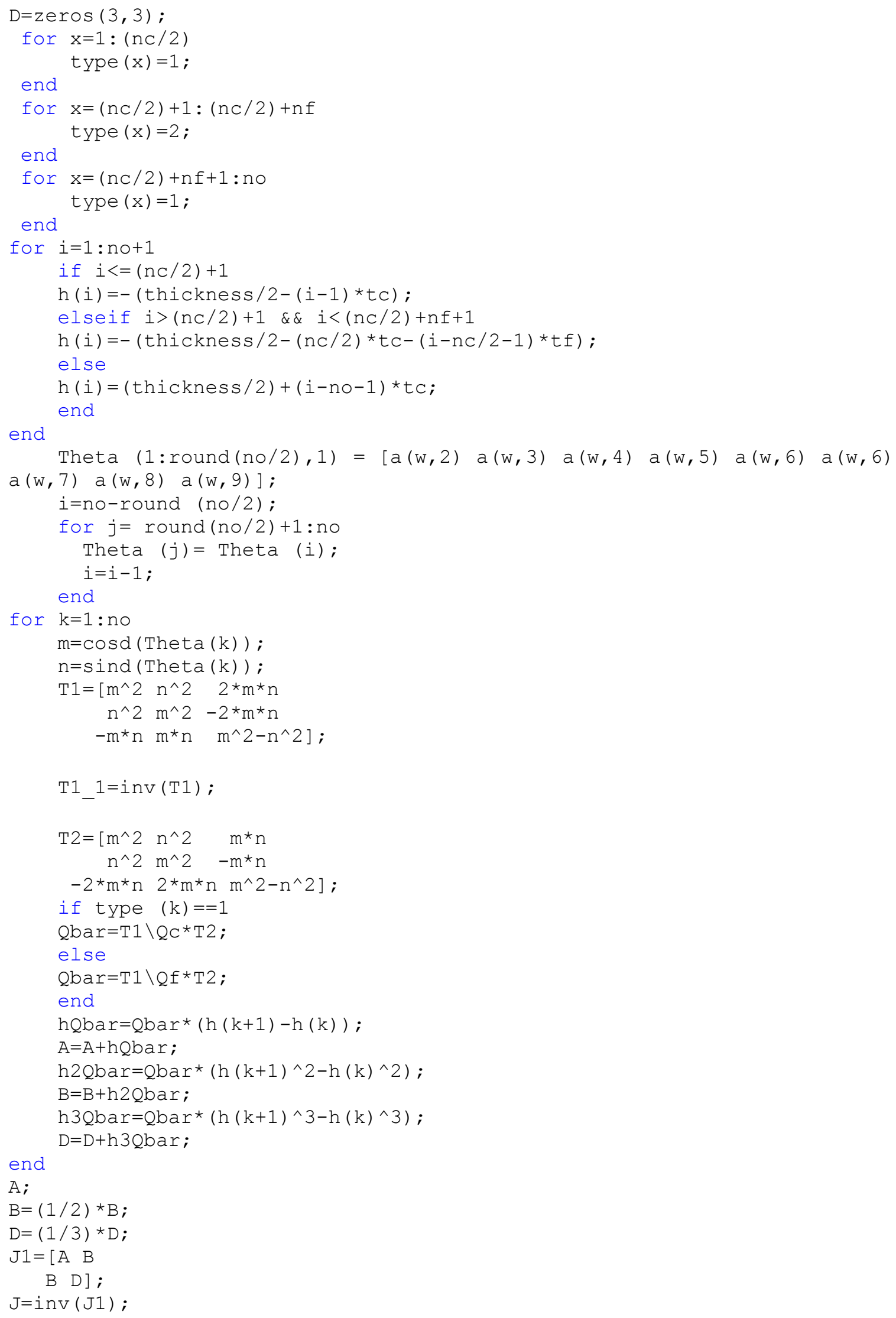




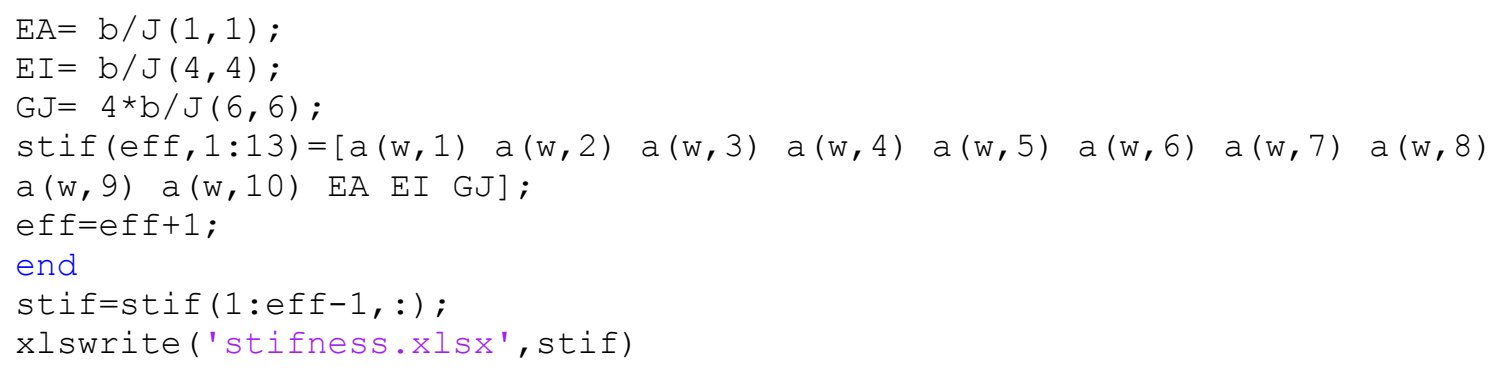




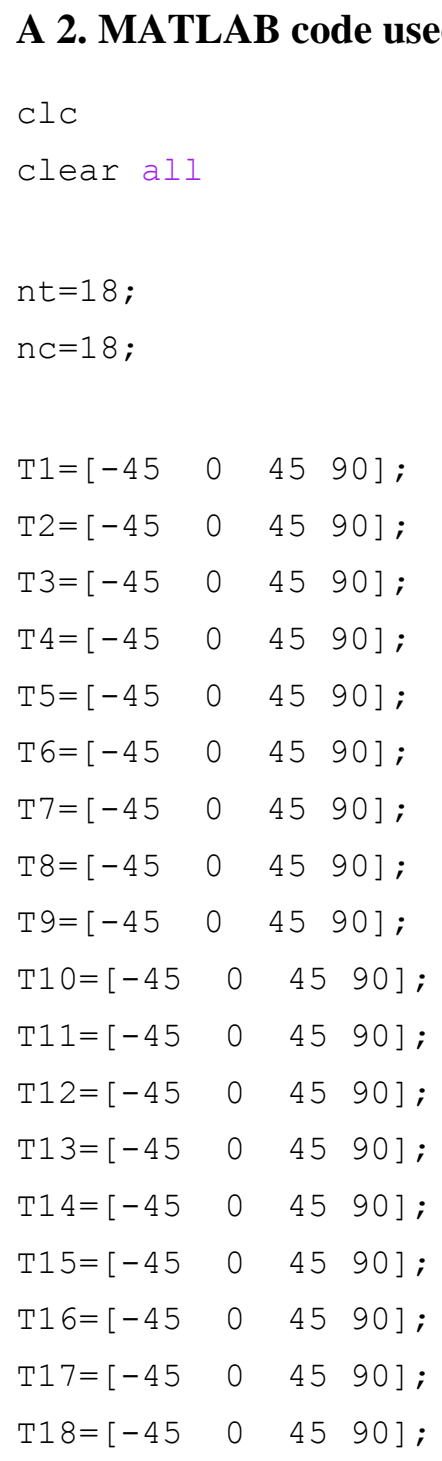

Theta2=zeros $(\mathrm{nc}, 1)$;

ROW=length ( $\mathrm{nc})$ *length $(\mathrm{T} 1)$ *length $(\mathrm{T} 2)$ *length $(\mathrm{T} 3)$ *length $(\mathrm{T} 4)$ *length (T5) *length ( $\mathrm{T} 6)$ *length $(\mathrm{T} 7)$ *length $(\mathrm{T} 8)$ *length $(\mathrm{T} 9)$ *length $(\mathrm{T} 10)$ *length $(\mathrm{T} 11) *$ length $(\mathrm{T} 12)$ *leng th $(\mathrm{T} 13) *$ length $(\mathrm{T} 14) *$ length $(\mathrm{T} 15) *$ length $(\mathrm{T} 16) *$ length $(\mathrm{T} 17) *$ length $(\mathrm{T} 18)$

OUT=zeros $(R O W, n t+1)$;

$\mathrm{cnt}=1$;

for $i=1$ : length (nc)

for $j=1$ : length (T1)

for $\mathrm{k}=1$ : length (T2)

for $l=1$ : length (T3)

for $\mathrm{m}=1$ : length (T4)

for $\mathrm{n}=1$ : length (T5) 


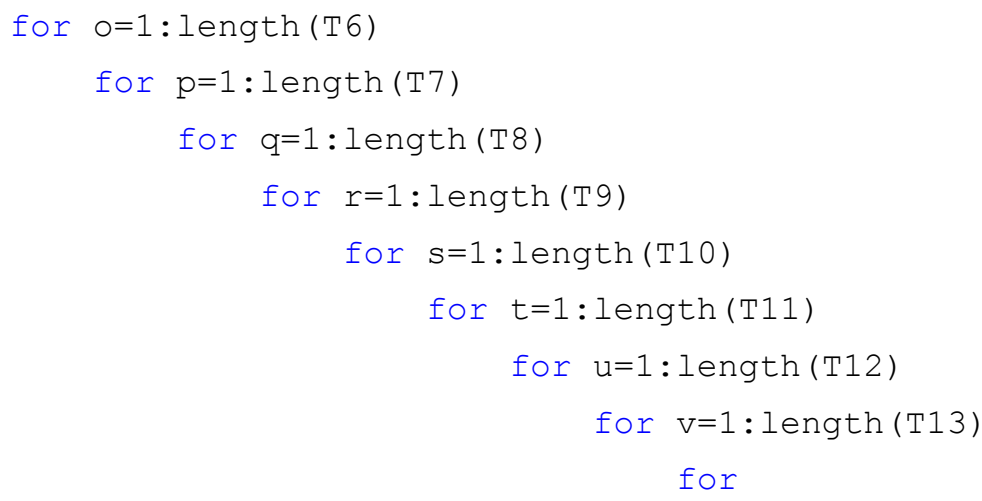




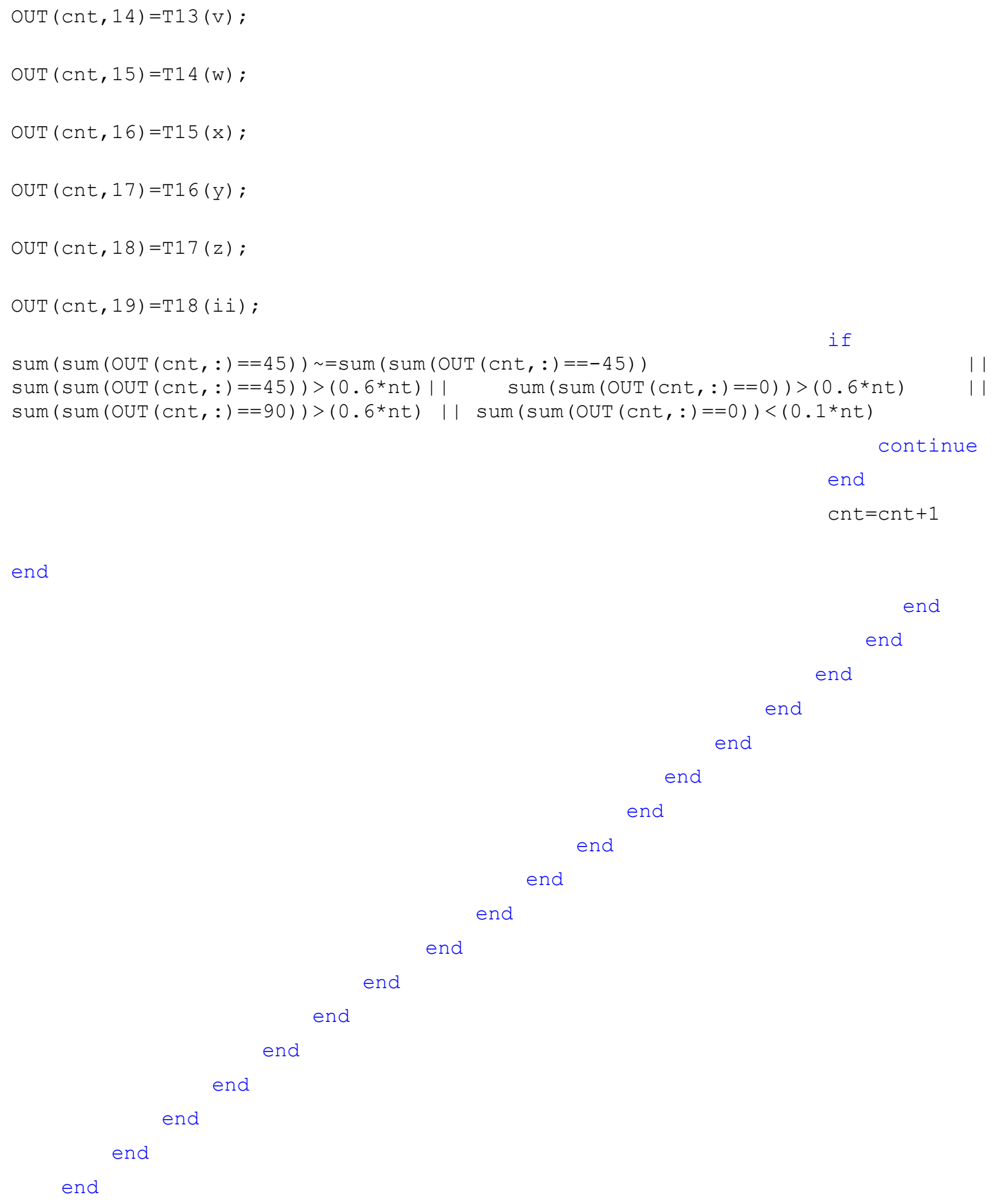




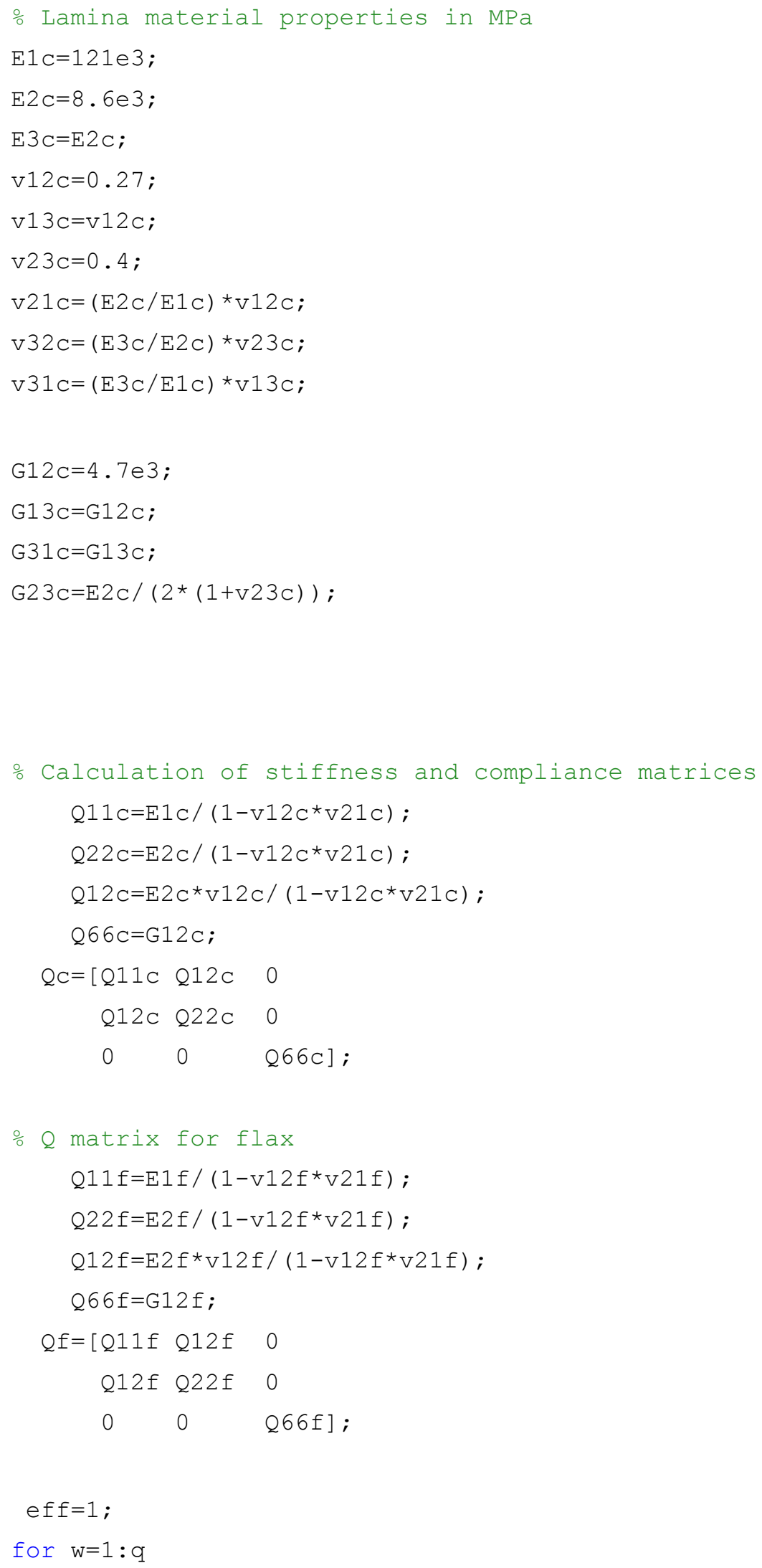




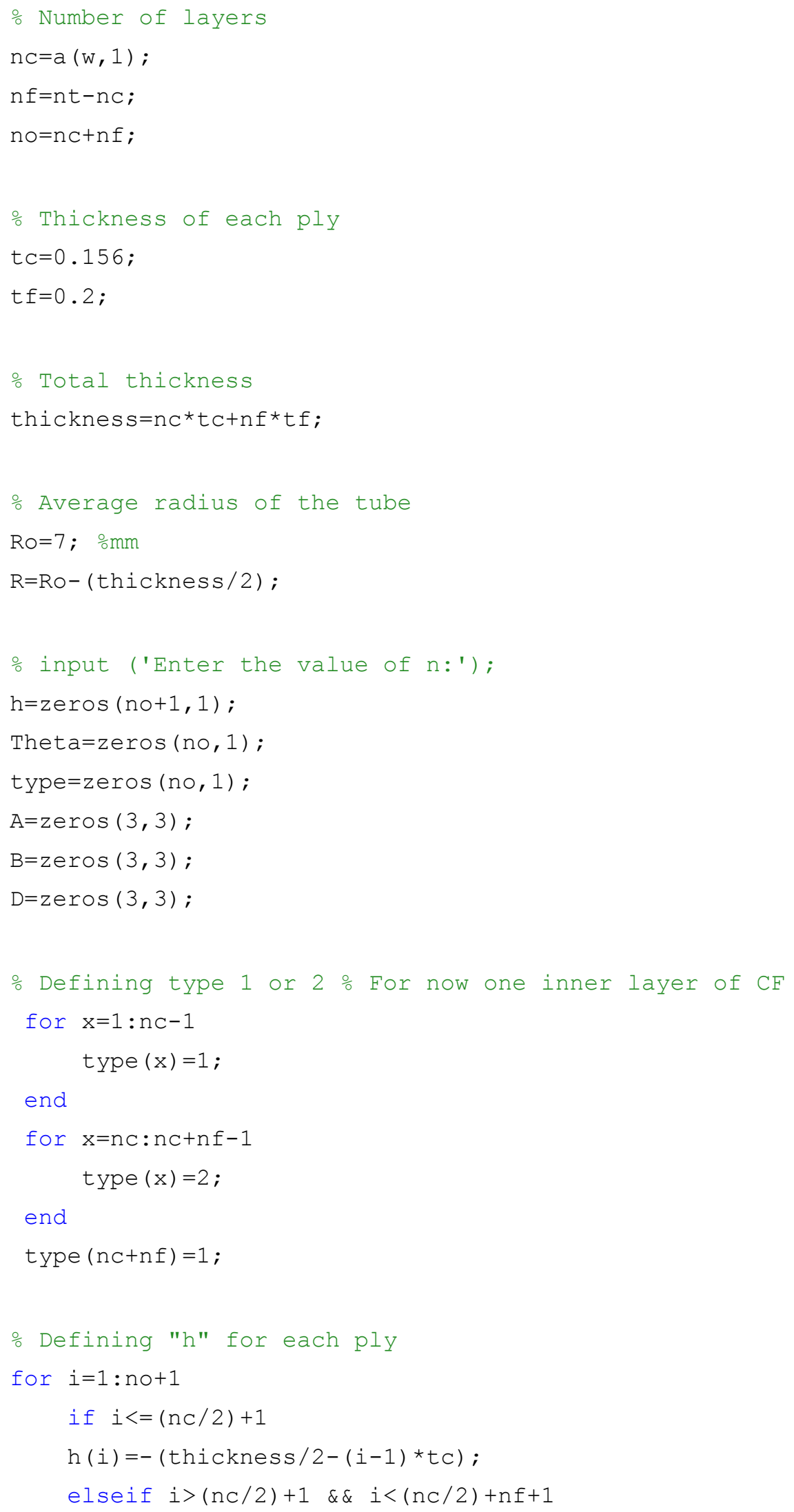




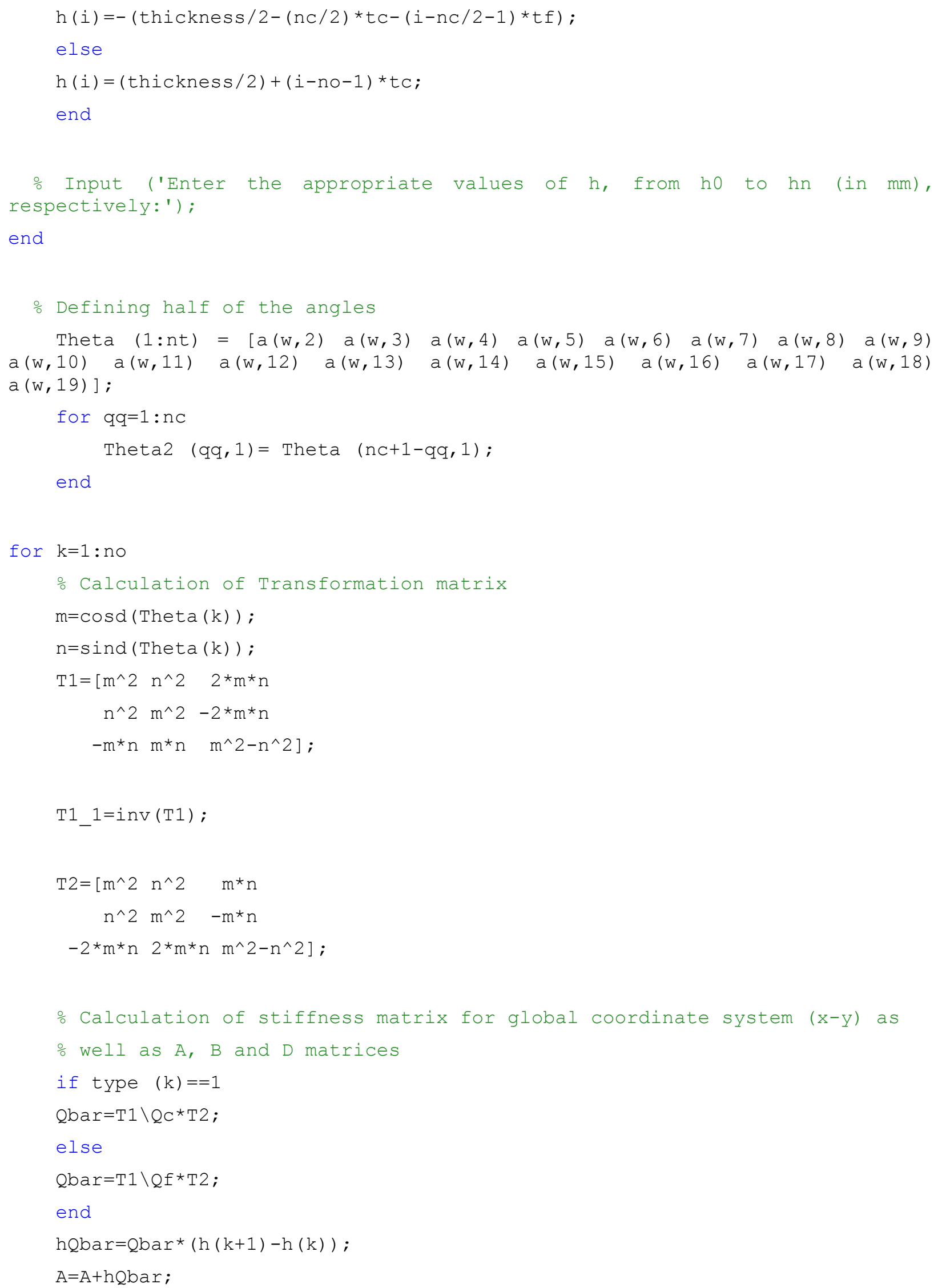




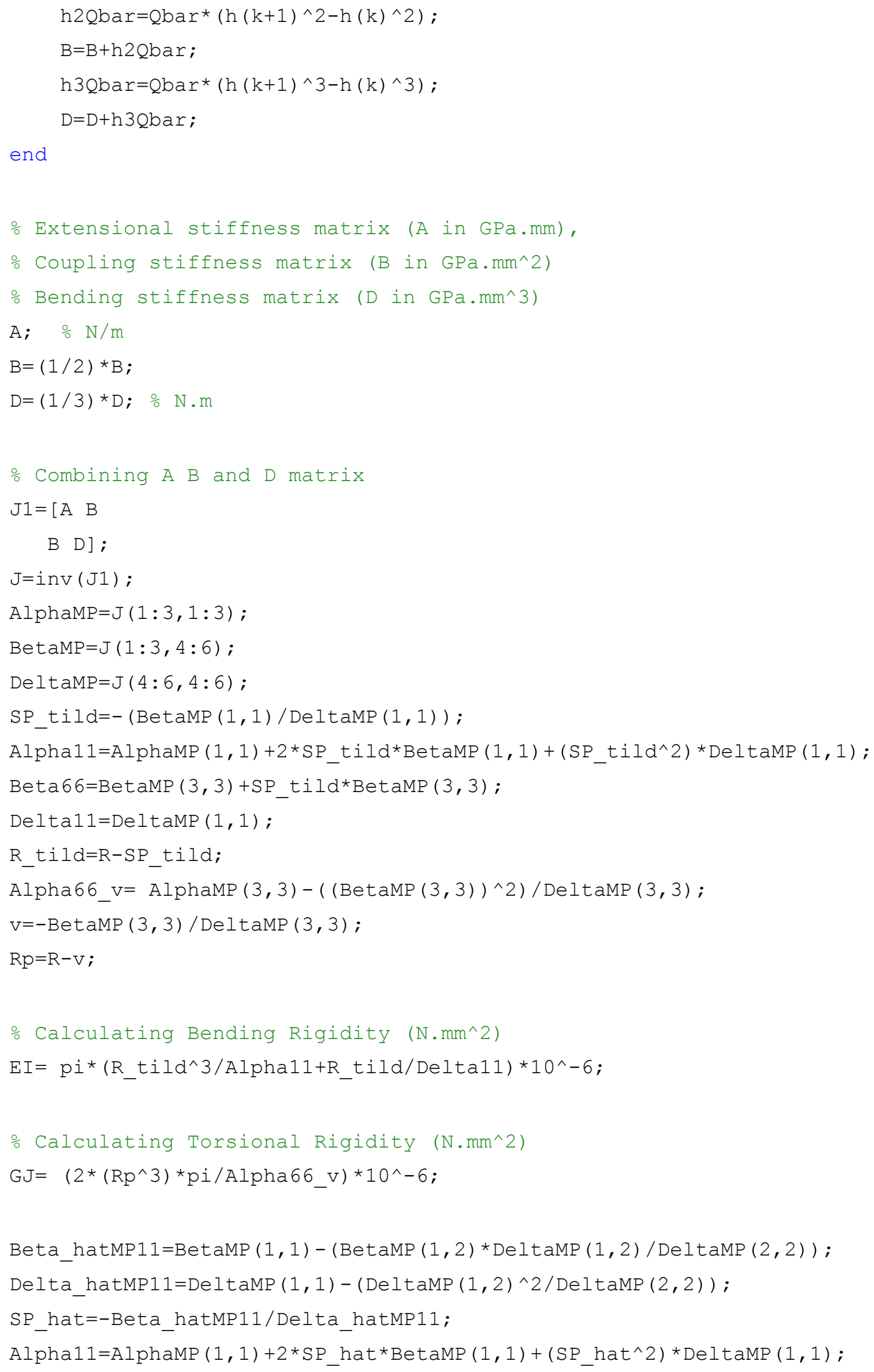




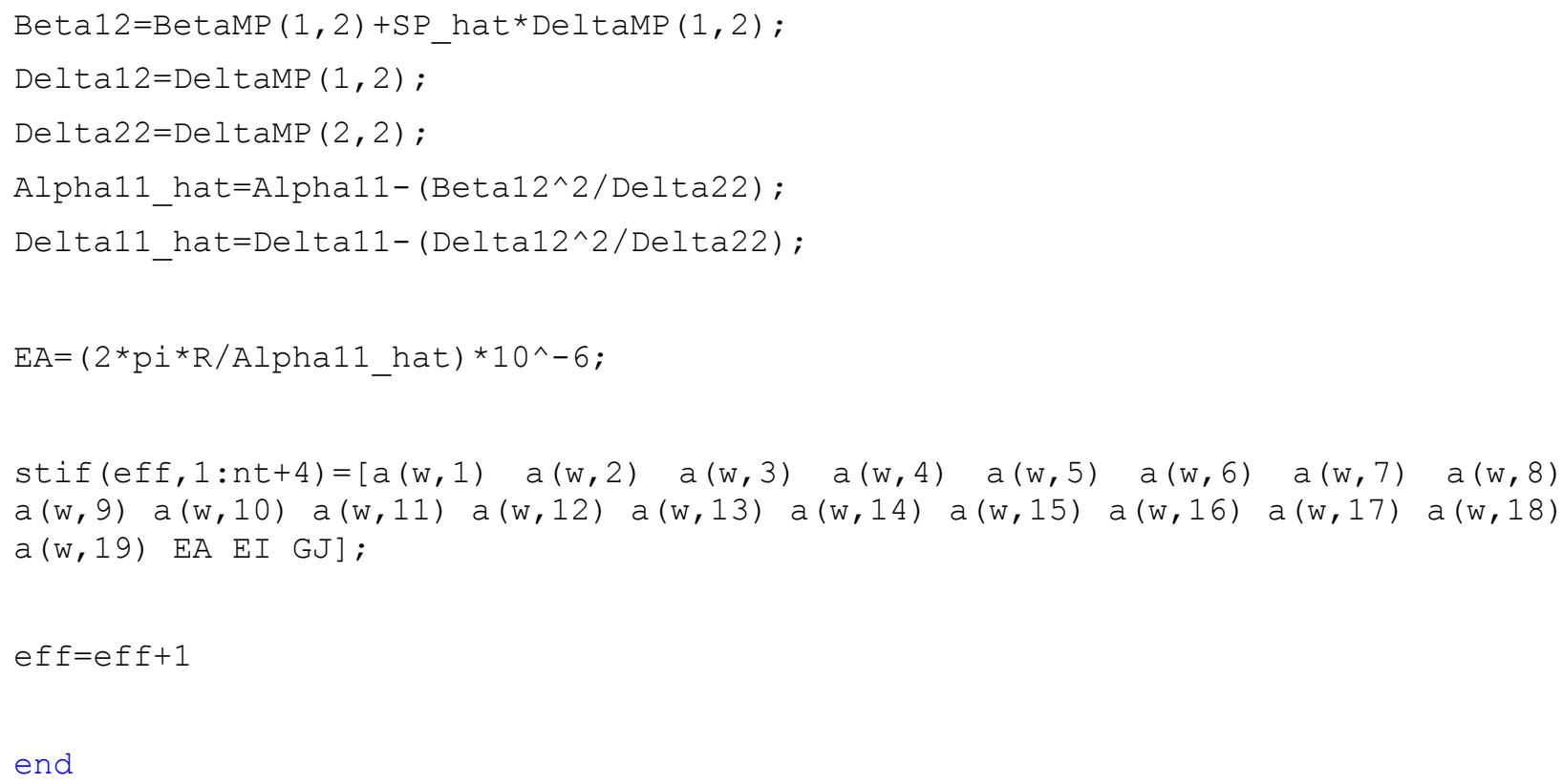




\section{REFERENCES}

[1] Montanini, R., and Filardi, V., 2010, "In vitro biomechanical evaluation of antegrade femoral nailing at early and late postoperative stages." Med Eng Phys, 32 (8), 889-897.

[2] Rockwood, C. A., Bucholz, R. W., Green, D. P., Court-Brown, C. M., Heckman, J. D., and Tornetta, P., 2010, Rockwood and Green's Fractures in Adults, Wolters Kluwer Health/Lippincott Williams \& Wilkins. Philadelphia.

[3] AAOS. Femur Shaft Fractures (Broken Thighbone). 2008.

http://orthoinfoaaosorg/topiccfm?topic=A00364.

[4] Braten, M., Terjesen, T., and Rossvoll, I., 1995, "Femoral shaft fractures treated by intramedullary nailing. A follow-up study focusing on problems related to the method." Injury, $26(6), 379-383$.

[5] Cheung, G., Zalzal, P., Bhandari, M., Spelt, J. K., and Papini, M., 2004, "Finite element analysis of a femoral retrograde intramedullary nail subject to gait loading." Med Eng Phys, 26 (2), 93-108.

[6] Frost, H. M., 1987, "Bone "mass" and the "mechanostat": a proposal." Anat Rec, 219 (1), 1-9.

[7] Bagheri, Z. Biomechanical development and analysis of a new carbonfiber/flax/epoxy plate for repairing femur fractures. 2014. Ryerson University, PhD Thesis, Toronto, Canada.

[8] Marsell, R., and Einhorn, T. A., 2011, "The biology of fracture healing." Injury, 42 (6), 551555.

[9] Augat, P., Burger, J., Schorlemmer, S., Henke, T., Peraus, M., and Claes, L., 2003, "Shear movement at the fracture site delays healing in a diaphyseal fracture model." J Orthop Res, 21 (6), 1011-1017.

[10] Epari, D. R., Kassi, J. P., Schell, H., and Duda, G. N., 2007, "Timely fracture-healing requires optimization of axial fixation stability." J Bone Joint Surg Am, 89 (7), 1575-1585.

[11] Mantripragada, V. P., Lecka-Czernik, B., Ebraheim, N. A., and Jayasuriya, A. C., 2013, "An overview of recent advances in designing orthopedic and craniofacial implants." J Biomed Mater Res A, 101 (11), 3349-3364. 
[12] Cifuentes, S. C., Frutos, E., González-Carrasco, J. L., Muñoz, M., Multigner, M., Chao, J., Benavente, R., and Lieblich, M., 2012, "Novel PLLA/magnesium composite for orthopedic applications: A proof of concept." Materials Letters, 74 (0), 239-242.

[13] Singh, V., 2014, Textbook of Anatomy Abdomen and Lower Limb, Elsevier Health Sciences APAC. New Delhi.

[14] Bontrager, K., 2013, e-Study Guide for Textbook of Radiographic Positioning and Related Anatomy, Cram101. www.justthefacts101.com

[15] Bonnick, S. L., 2009, Bone Densitometry in Clinical Practice: Application and Interpretation, Humana Press. New York.

[16] Wikipedia. Epiphysis. Last access 11-09-2015. en.wikipedia.org/wiki/Epiphysis.

[17] Cummings, S. R., Rubin, S. M., and Black, D., 1990, "The future of hip fractures in the United States. Numbers, costs, and potential effects of postmenopausal estrogen." Clin Orthop Relat Res, (252), 163-166.

[18] Singer, B. R., McLauchlan, G. J., Robinson, C. M., and Christie, J., 1998, "Epidemiology of fractures in 15,000 adults: the influence of age and gender." J Bone Joint Surg Br, 80 (2), 243248 .

[19] Court-Brown, C. M., and Caesar, B., 2006, "Epidemiology of adult fractures: A review." Injury, 37 (8), 691-697.

[20] Arneson, T. J., Melton, L. J., 3rd, Lewallen, D. G., and O'Fallon, W. M., 1988, "Epidemiology of diaphyseal and distal femoral fractures in Rochester, Minnesota, 1965-1984." Clin Orthop Relat Res, (234), 188-194.

[21] Court-Brown, C. M., Rimmer, S., Prakash, U., and McQueen, M. M., 1998, "The epidemiology of open long bone fractures." Injury, 29 (7), 529-534.

[22] Wolinsky, P. R., McCarty, E., Shyr, Y., and Johnson, K., 1999, "Reamed intramedullary nailing of the femur: 551 cases." J Trauma, 46 (3), 392-399.

[23] Egol, K. A., Koval, K. J., and Zuckerman, J. D., 2010, Handbook of Fractures, Wolters Kluwer/Lippincott Williams \& Wilkins Health. Philadelphia. 
[24] Winquist, R. A., and Hansen, S. T., Jr., 1980, "Comminuted fractures of the femoral shaft treated by intramedullary nailing." Orthop Clin North Am, 11 (3), 633-648.

[25] Winquist, R. A., Hansen, S. T., Jr., and Clawson, D. K., 1984, "Closed intramedullary nailing of femoral fractures. A report of five hundred and twenty cases." J Bone Joint Surg Am, $66(4), 529-539$.

[26] Koval, K. J., and Zuckerman, J. D., 2006, Handbook of Fractures, Lippincott Williams \& Wilkins. Philadelphia.

[27] Marsh, J. L., Slongo, T. F., Agel, J., Broderick, J. S., Creevey, W., DeCoster, T. A., Prokuski, L., Sirkin, M. S., Ziran, B., Henley, B., and Audige, L., 2007, "Fracture and dislocation classification compendium - 2007: Orthopaedic Trauma Association classification, database and outcomes committee." J Orthop Trauma, 21 (10 Suppl), S1-133.

[28] Harwood, P. J., Giannoudis, P. V., Probst, C., Krettek, C., and Pape, H. C., 2006, "The risk of local infective complications after damage control procedures for femoral shaft fracture." $\mathbf{J}$ Orthop Trauma, 20 (3), 181-189.

[29] Aro, H. T., and Chao, E. Y., 1993, "Bone-healing patterns affected by loading, fracture fragment stability, fracture type, and fracture site compression." Clin Orthop Relat Res, (293), 817.

[30] Augat, P., Simon, U., Liedert, A., and Claes, L., 2005, "Mechanics and mechano-biology of fracture healing in normal and osteoporotic bone." Osteoporos Int, 16 Suppl 2 S36-43.

[31] Wang, G., Pan, T., Peng, X., and Wang, J., 2008, "A new intramedullary nailing device for the treatment of femoral shaft fractures: a biomechanical study." Clin Biomech (Bristol, Avon), 23 (3), 305-312.

[32] Browner, B. D., 2009, Skeletal Trauma: Basic Science, Management, and Reconstruction, Saunders/Elsevier. Philadelphia.

[34] Burstein, A. H., Zika, J. M., Heiple, K. G., and Klein, L., 1975, "Contribution of collagen and mineral to the elastic-plastic properties of bone." J Bone Joint Surg Am, 57 (7), 956-961. [35] Carter, D. R., and Hayes, W. C., 1977, "The compressive behavior of bone as a two-phase porous structure." J Bone Joint Surg Am, 59 (7), 954-962. 
[36] Ascenzi, A., and Bonucci, E., 1967, "The tensile properties of single osteons." Anat Rec, 158 (4), 375-386.

[37] Ascenzi, A., and Bonucci, E., 1968, "The compressive properties of single osteons." Anat Rec, 161 (3), 377-391.

[38] Ascenzi, A., and Bonucci, E., 1972, "The shearing properties of single osteons." Anat Rec, $172(3), 499-510$.

[39] Evans, F. G., and Vincentelli, R., 1969, "Relation of collagen fiber orientation to some mechanical properties of human cortical bone." J Biomech, 2 (1), 63-71.

[40] Bartley, M. H., Jr., Arnold, J. S., Haslam, R. K., and Jee, W. S., 1966, "The relationship of bone strength and bone quantity in health, disease, and aging." J Gerontol, 21 (4), 517-521.

[41] Burstein, A. H., Reilly, D. T., and Martens, M., 1976, "Aging of bone tissue: mechanical properties." J Bone Joint Surg Am, 58 (1), 82-86.

[42] Perren, S. M., 1979, "Physical and biological aspects of fracture healing with special reference to internal fixation." Clin Orthop Relat Res, (138), 175-196.

[43] Aro, H. T., Wipperman, B. W., Hodgson, S. F., Wahner, H. W., Lewallen, D. G., and Chao, E. Y. S., 1989, "Prediction of properties of fracture callus by measurement of mineral density using micro-bone densitometry." Journal of Bone and Joint Surgery - Series A, 71 (7), 10201030.

[44] White Iii, A. A., Panjabi, M. M., and Southwick, W. O., 1977, "The four biomechanical stages of fracture repair." Journal of Bone and Joint Surgery - Series A, 59 (2), 188-192.

[45] Lieberman, J. R., and Friedlaender, G. E., 2007, Bone Regeneration and Repair: Biology and Clinical Applications, Humana Press. Danvers.

[46] White, A. A., 3rd, Panjabi, M. M., and Southwick, W. O., 1977, "Effects of compression and cyclical loading on fracture healing--a quantitative biomechanical study." J Biomech, 10 (4), 233-239.

[47] Woodard, P. L., Self, J., Calhoun, J., Tencer, A. F., and Evans, E. B., 1987, "The effect of implant axial and torsional stiffness on fracture healing." J Orthop Trauma, 1 (4), 331-340. 
[48] Poitout, D. G., 2004, Biomechanics and Biomaterials in Orthopedics, Springer-Verlag. London.

[49] Mow, V. C., and Huiskes, R., 2005, Basic Orthopaedic Biomechanics \& Mechano-biology, Lippincott Williams \& Wilkins. Philadelphia.

[50] Sarmiento, A., McKellop, H. A., Llinas, A., Park, S. H., Lu, B., Stetson, W., and Rao, R., 1996, "Effect of loading and fracture motions on diaphyseal tibial fractures." J Orthop Res, 14 (1), 80-84.

[51] Park, S. H., O'Connor, K., McKellop, H., and Sarmiento, A., 1998, "The influence of active shear or compressive motion on fracture-healing." J Bone Joint Surg Am, 80 (6), 868-878.

[52] Bishop, N. E., Van Rhijn, M., Tami, I., Corveleijn, R., Schneider, E., and Ito, K., 2006, "Shear does not necessarily inhibit bone healing." Clin Orthop Relat Res, (443), 307-314. [53] Eveleigh, R. J., 1995, "A review of biomechanical studies of intramedullary nails." Med Eng Phys, 17 (5), 323-331.

[54] Wolinsky, P., Tejwani, N., Richmond, J. H., Koval, K. J., Egol, K., and Stephen, D. J., 2002, "Controversies in intramedullary nailing of femoral shaft fractures." Instr Course Lect, 51 291-303.

[55] Tonino, A. J., Davidson, C. L., Klopper, P. J., and Linclau, L. A., 1976, "Protection from stress in bone and its effects. Experiments with stainless steel and plastic plates in dogs." J Bone Joint Surg Br, 58 (1), 107-113.

[56] Bougherara, H., Zdero, R., Miric, M., Shah, S., Hardisty, M., Zalzal, P., and Schemitsch, E. H., 2009, "The biomechanics of the T2 femoral nailing system: a comparison of synthetic femurs withfinite element analysis." Proc Inst Mech Eng H, 223 (3), 303-314.

[57] Foux, A., Yeadon, A. J., and Uhthoff, H. K., 1997, "Improved fracture healing with less rigid plates. A biomechanical study in dogs." Clin Orthop Relat Res, (339), 232-245.

[58] Fujihara, K., Huang, Z. M., Ramakrishna, S., Satknanantham, K., and Hamada, H., 2003, "Performance study of braided carbon/PEEK composite compression bone plates." Biomaterials, 24 (15), 2661-2667. 
[59] Perez, A., Mahar, A., Negus, C., Newton, P., and Impelluso, T., 2008, "A computational evaluation of the effect of intramedullary nail material properties on the stabilization of simulated femoral shaft fractures." Med Eng Phys, 30 (6), 755-760.

[60] Sha, M., Guo, Z., Fu, J., Li, J., Yuan, C. F., Shi, L., and Li, S. J., 2009, "The effects of nail rigidity on fracture healing in rats with osteoporosis." Acta Orthop, 80 (1), 135-138.

[61] Campbell, M., Denault, J., Yahia, L. H., and Bureau, M. N., 2008, "CF/PA12 composite femoral stems: Manufacturing and properties." Compos Part A-Appl S, 39 (5), 796-804.

[62] Jacobsson, S. A., Djerf, K., Gillquist, J., Hammerby, S., and Ivarsson, I., 1993, "A prospective comparison of Butel and PCA hip arthroplasty." J Bone Joint Surg Br, 75 (4), 624629.

[63] Jakim, I., Barlin, C., and Sweet, M. B. E., 1988, "RM isoelastic total hip arthroplasty: A review of 34 cases." J Arthroplasty, 3 (3), 191-199.

[64] Bradley, J. S., Hastings, G. W., and Johnson-Nurse, C., 1980, "Carbon fibre reinforced epoxy as a high strength, low modulus material for internal fixation plates." Biomaterials, 1 (1), $38-40$.

[65] Tayton, K., Johnson-Nurse, C., McKibbin, B., Bradley, J., and Hastings, G., 1982, "The use of semi-rigid carbon-fibre-reinforced plastic plates for fixation of human fractures. Results of preliminary trials." J Bone Joint Surg Br, 64 (1), 105-111.

[66] Woo, S. L., Akeson, W. H., Levenetz, B., Coutts, R. D., Matthews, J. V., and Amiel, D., 1974, "Potential application of graphite fiber and methyl methacrylate resin composites as internal fixation plates." J Biomed Mater Res, 8 (5), 321-338.

[67] Blackwood, D. J., Chua, A. W. C., Seah, K. H. W., Thampuran, R., and Teoh, S. H., 2000, "Corrosion behaviour of porous titanium-graphite composites designed for surgical implants." Corros Sci, 42 (3), 481-503.

[68] Boudeau, N., Liksonov, D., Barriere, T., Maslov, L., and Gelin, J. C., 2012, "Composite based on polyetheretherketone reinforced with carbon fibres, an alternative to conventional materials for femoral implant: Manufacturing process and resulting structural behaviour." Mater Design, 40 (0), 148-156. 
[69] Erkmen, E., Meriç, G., Kurt, A., Tunç, Y., and Eser, A., 2011, "Biomechanical comparison of implant retained fixed partial dentures with fiber reinforced composite versus conventional metal frameworks: A 3D FEA study." J Mech Behav Biomed Mater, 4 (1), 107-116.

[70] Hao, L., Dadbakhsh, S., Seaman, O., and Felstead, M., 2009, "Selective laser melting of a stainless steel and hydroxyapatite composite for load-bearing implant development." J Mater Process Tech, 209 (17), 5793-5801.

[71] Hastings, G. W., 1978, "Carbon fibre composites for orthopaedic implants." Composites, 9 (3), 193-197.

[72] Meriç, G., Erkmen, E., Kurt, A., Eser, A., and Çelik, G., 2010, "Biomechanical evaluation of a fiber-reinforced composite prosthesis supported by implants with and without a microthread collar design." J Dent Sci, 5 (4), 201-208.

[73] Steinberg, E. L., Rath, E., Shlaifer, A., Chechik, O., Maman, E., and Salai, M., 2013, "Carbon fiber reinforced PEEK Optima-A composite material biomechanical properties and wear/debris characteristics of CF-PEEK composites for orthopedic trauma implants." J Mech Behav Biomed Mater, 17 (0), 221-228.

[74] Zhao, D. S., Moritz, N., Laurila, P., Mattila, R., Lassila, L. V. J., Strandberg, N., Mäntylä, T., Vallittu, P. K., and Aro, H. T., 2009, "Development of a multi-component fiber-reinforced composite implant for load-sharing conditions." Med Eng Phys, 31 (4), 461-469.

[75] Zilberman, M., 2007, "Novel composite fiber structures to provide drug/protein delivery for medical implants and tissue regeneration." Acta Biomater, 3 (1), 51-57.

[76] Ramakrishna, S., Mayer, J., Wintermantel, E., and Leong, K. W., 2001, "Biomedical applications of polymer-composite materials: a review." Compos Sci Technol, 61 (9), 11891224.

[77] Suchý, T., Balík, K., Sedláček, R., Sucharda, Z., Sochor, M., Prokop, J., Beneš, J., and Křena, J., 2011, "Radiolucent composites providing high resistance against sterilization decomposition." Ceramics - Silikaty, 55 (4), 401-409.

[78] Friedrich, K., Fakirov, S., and Zhang, Z., 2005, Polymer Composites: From Nano- to Macro-Scale, Springer. New York. 
[79] Ali, M. S., French, T. A., Hastings, G. W., Rae, T., Rushton, N., Ross, E. R. S., and WynnJones, C. H., 1990, "Carbon fibre composite bone plates. Development, evaluation and early clinical experience." J Bone Joint Surg Br, 72 (4), 586-591.

[80] Howling, G. I., Ingham, E., Sakoda, H., Stewart, T. D., Fisher, J., Antonarulrajah, A., Appleyard, S., and Rand, B., 2004, "Carbon-carbon composite bearing materials in hip arthroplasty: analysis of wear and biological response to wear debris." J Mater Sci Mater Med, 15 (1), 91-98.

[81] Bagheri, Z. S., El Sawi, I., Schemitsch, E. H., Zdero, R., and Bougherara, H., 2013, "Biomechanical properties of an advanced new carbon/flax/epoxy composite material for bone plate applications." J Mech Behav Biomed Mater, 20 398-406.

[82] Terjesen, T., and Apalset, K., 1988, "The influence of different degrees of stiffness of fixation plates on experimental bone healing." J Orthop Res, 6 (2), 293-299.

[83] Kenwright, J., and Goodship, A. E., 1989, "Controlled mechanical stimulation in the treatment of tibial fractures." Clin Orthop Relat Res, (241), 36-47.

[84] Tayton, K., and Bradley, J., 1983, "How stiff should semi-rigid fixation of the human tibia be? A clue to the answer." J Bone Joint Surg Br, 65 (3), 312-315.

[85] Woo, S. L., Lothringer, K. S., Akeson, W. H., Coutts, R. D., Woo, Y. K., Simon, B. R., and Gomez, M. A., 1984, "Less rigid internal fixation plates: historical perspectives and new concepts." J Orthop Res, 1 (4), 431-449.

[86] Skirving, A. P., Day, R., Macdonald, W., and McLaren, R., 1987, "Carbon fiber reinforced plastic (CFRP) plates versus stainless steel dynamic compression plates in the treatment of fractures of the tibiae in dogs." Clin Orthop Relat Res, (224), 117-124.

[87] Huang, Z.-M., and Fujihara, K., 2005, "Stiffness and strength design of composite bone plates." Compos Sci Technol, 65 (1), 73-85.

[88] Ganesh, V. K., Ramakrishna, K., and Ghista, D., 2005, "Biomechanics of bone-fracture fixation by stiffness-graded plates in comparison with stainless-steel plates." BioMedical Engineering OnLine, 4 (1), 1-15.

[89] Veerabagu, S., Fujihara, K., Dasari, G. R., and Ramakrishna, S., 2003, "Strain distribution analysis of braided composite bone plates." Compos Sci Technol, 63 (3-4), 427-435. 
[90] Siddiqui, F. S., Shah, S., Nicayenzi, B., Schemitsch, E. H., Zdero, R., and Bougherara, H., 2014, "Biomechanical analysis using infrared thermography of a traditional metal plate versus a carbon fibre/epoxy plate for Vancouver B1 femur fractures." Proc Inst Mech Eng H, 228 (1), 107-113.

[91] Bagheri, Z. S., Tavakkoli Avval, P., Bougherara, H., Aziz, M. S., Schemitsch, E. H., and Zdero, R., 2014, "Biomechanical analysis of a new carbon fiber/flax/epoxy bone fracture plate shows less stress shielding compared to a standard clinical metal plate." J Biomech Eng, 136 (9), 091002.

[92] Kim, S.-H., Chang, S.-H., and Jung, H.-J., 2010, "The finite element analysis of a fractured tibia applied by composite bone plates considering contact conditions and time-varying properties of curing tissues." Compos Struct, 92 (9), 2109-2118.

[93] Kim, H.-J., Kim, S.-H., and Chang, S.-H., 2011, "Finite element analysis using interfragmentary strain theory for the fracture healing process to which composite bone plates are applied." Compos Struct, 93 (11), 2953-2962.

[94] Bagheri, Z. S., El Sawi, I., Bougherara, H., and Zdero, R., 2014, "Biomechanical fatigue analysis of an advanced new carbon fiber/flax/epoxy plate for bone fracture repair using conventional fatigue tests and thermography." J Mech Behav Biomed Mater, 35 27-38.

[95] Moritz, N., Strandberg, N., Zhao, D. S., Mattila, R., Paracchini, L., Vallittu, P. K., and Aro, H. T., 2014, "Mechanical properties and in vivo performance of load-bearing fiber-reinforced composite intramedullary nails with improved torsional strength." J Mech Behav Biomed Mater, $40(0), 127-139$.

[96] Morawska-Chochół, A., Chłopek, J., Szaraniec, B., Domalik-Pyzik, P., Balacha, E., Boguń, M., and Kucharski, R., 2015, "Influence of the intramedullary nail preparation method on nail's mechanical properties and degradation rate." Mater Sci Eng C, 51 99-106.

[97] Metzinger, A. J., Stuart G. R. , and Yambor, J. N., 2009, "Composite intramedullary nail" Patent number: US 2009/0088752 A1.

[98] Arazi, M., Ogun, T. C., Oktar, M. N., Memik, R., and Kutlu, A., 2001, "Early weightbearing after statically locked reamed intramedullary nailing of comminuted femoral fractures: is it a safe procedure?." J Trauma, 50 (4), 711-716. 
[99] Brumback, R. J., Toal, T. R., Jr., Murphy-Zane, M. S., Novak, V. P., and Belkoff, S. M., 1999, "Immediate weight-bearing after treatment of a comminuted fracture of the femoral shaft with a statically locked intramedullary nail." J Bone Joint Surg Am, 81 (11), 1538-1544.

[100] Shih, K. S., Hsu, C. C., and Hsu, T. P., 2012, "A biomechanical investigation of the effects of static fixation and dynamization after interlocking femoral nailing: a finite element study." J Trauma Acute Care Surg, 72 (2), E46-53.

[101] Kollár, L. P., and Springer, G. S., 2003, Mechanics of Composite Structures, Cambridge University Press. Cambridge.

[102] Agarwal, B. D., Broutman, L. J., and Chandrashekhara, K., 2006, Analysis and Performance of Fiber Composites, John Wiley \& Sons. New Jersey.

[103] Bailie, J. A., Ley, R. P., and Pasricha, A. A summary and review of composite laminate design guidelines. Technical report NASA; 1997; NAS1-19347.

[104] Dubov, A., Kim, S. Y., Shah, S., Schemitsch, E. H., Zdero, R., and Bougherara, H., 2011, "The biomechanics of plate repair of periprosthetic femur fractures near the tip of a total hip implant: the effect of cable-screw position." Proc Inst Mech Eng H, 225 (9), 857-865.

[105] Gardner, T. N., Stoll, T., Marks, L., Mishra, S., and Knothe Tate, M., 2000, "The influence of mechanical stimulus on the pattern of tissue differentiation in a long bone fracture - an FEM study." J Biomech, 33 (4), 415-425.

[106] Ebrahimi, H., Rabinovich, M., Vuleta, V., Zalcman, D., Shah, S., Dubov, A., Roy, K., Siddiqui, F. S., E, H. S., Bougherara, H., and Zdero, R., 2012, "Biomechanical properties of an intact, injured, repaired, and healed femur: an experimental and computational study." J Mech Behav Biomed Mater, 16 121-135.

[107] Holt, J. M., Gibson, C., Ho, C. Y., Information, P. U. C. f., Analysis, N. D., and Synthesis, 1999, Structural Alloys Handbook, CINDAS/Purdue University.

[108] Pacific Research Laboratories. Biomechnical Test Material. 2014. www.sawbones.com.

[109] Welsch, G., Boyer, R., and Collings, E. W., 1993, Materials Properties Handbook:

Titanium Alloys, ASM International. 
[110] Duda, G. N., Schneider, E., and Chao, E. Y., 1997, "Internal forces and moments in the femur during walking." J Biomech, 30 (9), 933-941.

[111] Duda, G. N., Heller, M., Albinger, J., Schulz, O., Schneider, E., and Claes, L., 1998, "Influence of muscle forces on femoral strain distribution." J Biomech, 31 (9), 841-846.

[112] Bitsakos, C., Kerner, J., Fisher, I., and Amis, A. A., 2005, "The effect of muscle loading on the simulation of bone remodelling in the proximal femur." J Biomech, 38 (1), 133-139.

[113] Bartel, D. L., Davy, D. T., and Keaveny, T. M., 2006, Orthopaedic biomechanics: mechanics and design in musculoskeletal systems, Pearson/Prentice Hall. New Jersey.

[114] Samiezadeh, S., Tavakkoli Avval, P., Fawaz, Z., and Bougherara, H., 2014, "Biomechanical assessment of composite versus metallic intramedullary nailing system in femoral shaft fractures: A finite element study." Clin Biomech (Bristol, Avon), 29 (7), 803-810. [115] Viceconti, M., Olsen, S., Nolte, L. P., and Burton, K., 2005, "Extracting clinically relevant data from finite element simulations." Clin Biomech (Bristol, Avon), 20 (5), 451-454.

[116] Uhthoff, H. K., Poitras, P., and Backman, D. S., 2006, "Internal plate fixation of fractures: short history and recent developments." J Orthop Sci, 11 (2), 118-126.

[117] Aro, H. T., Wahner, H. T., and Chao, E. Y., 1991, "Healing patterns of transverse and oblique osteotomies in the canine tibia under external fixation." J Orthop Trauma, 5 (3), 351364.

[118] El Sawi, I., Bougherara, H., Zitoune, R., and Fawaz, Z., 2014, "Influence of the Manufacturing Process on the Mechanical Properties of Flax/Epoxy Composites." J Biobased Mater Bio, 8 (1), 69-76.

[119] Papini, M., Zdero, R., Schemitsch, E. H., and Zalzal, P., 2007, "The biomechanics of human femurs in axial and torsional loading: comparison of finite element analysis, human cadaveric femurs, and synthetic femurs." J Biomech Eng, 129 (1), 12-19.

[120] Cristofolini, L., Viceconti, M., Cappello, A., and Toni, A., 1996, "Mechanical validation of whole bone composite femur models." J Biomech, 29 (4), 525-535.

[121] Frankel, V. H., and Nordin, M., 1980, Basic biomechanics of the skeletal system, Lea \& Febiger. Philadelphia. 
[122] Kim, C., and White, S. R., 1996, "Analysis of thick hollow composite beams under general loadings." Compos Struct, 34 (3), 263-277.

[123] Shadmehri, F., Derisi, B., and Hoa, S. V., 2011, "On bending stiffness of composite tubes." Compos Struct, 93 (9), 2173-2179.

[124] Chun, H. J., Park, M. J., and Byun, J. H., 2006, "Behaviors of CAS and CUS thick-walled channel composite beams." Int J Mod Phys B, 20 (25-27), 4016-4021.

[125] Kim, S. K., Lee, K. W., and Song, O., 2011, "Dynamic responses of composite H-beams with different elastic couplings." J Mech Sci Technol, 25 (10), 2505-2517.

[126] Samiezadeh, S., Fawaz, Z., and Bougherara, H., 2016, "Biomechanical properties of a structurally optimized carbon-fibre/epoxy intramedullary nail for femoral shaft fracture fixation." J Mech Behav Biomed Mater, 56 87-97.

[127] Librescu, L., and Song, O., 2006, Thin-Walled Composite Beams: Theory and Application, Springer. Dordrecht.

[128] Shadmehri, F., Haddadpour, H., and Kouchakzadeh, M. A., 2007, "Flexural-torsional behavior of thin-walled composite beams with closed cross-section." Thin Wall Struct, 45 (7-8), 699-705.

[129] Jolicoeur, C., and Cardou, A., 1994, "Analytical solution for bending of coaxial orthotropic cylinders." J Eng Mech, 120 (12), 2556-2574.

[130] Kim, C., and White, S. R., 1997, "Thick-walled composite beam theory including 3-d elastic effects and torsional warping." Int J Solids Struct, 34 (31-32), 4237-4259.

[131] Reddy, J. N., 1984, "A simple higher-order theory for laminated composite plates." J Appl Mech, 51 (4), 745-752.

[132] Vasiliev, V. V., and Morozov, E., 2013, Advanced Mechanics of Composite Materials and Structural Elements, Elsevier Science. Oxford.

[133] Vinson, J. R., 1993, The Behavior of Shells Composed of Isotropic and Composite Materials, Springer. Dordrecht.

[134] Heiner, A. D., 2008, "Structural properties of fourth-generation composite femurs and tibias." J Biomech, 41 (15), 3282-3284. 
[135] Samiezadeh, S., Tavakkoli Avval, P., Fawaz, Z., and Bougherara, H., 2015, "On optimization of a composite bone plate using the selective stress shielding approach." J Mech Behav Biomed Mater, 42 138-153.

[136] Tavakkoli Avval, P., Klika, V., and Bougherara, H., 2014, "Predicting Bone Remodeling in Response to Total Hip Arthroplasty: Computational Study Using Mechanobiochemical Model." J Biomech Eng, 136 (5), 051002-051002.

[137] Li, M. G., Rohrl, S. M., Wood, D. J., and Nivbrant, B., 2007, "Periprosthetic changes in bone mineral density in 5 stem designs 5 years after cemented total hip arthroplasty. No relation to stem migration." J Arthroplasty, 22 (5), 689-691.

[139] Allen, J. C., Jr., Lindsey, R. W., Hipp, J. A., Gugala, Z., Rianon, N., and LeBlanc, A., 2008, "The effect of retained intramedullary nails on tibial bone mineral density." Clin Biomech (Bristol, Avon), 23 (6), 839-843.

[140] Eyres, K. S., and Kanis, J. A., 1995, "Bone loss after tibial fracture. Evaluated by dualenergy X-ray absorptiometry." J Bone Joint Surg Br, 77 (3), 473-478.

[141] Kapp, W., Lindsey, R. W., Noble, P. C., Rudersdorf, T., and Henry, P., 2000, "Long-term residual musculoskeletal deficits after femoral shaft fractures treated with intramedullary nailing." J Trauma, 49 (3), 446-449.

[142] Kaiser, M. M., Wessel, L. M., Zachert, G., Stratmann, C., Eggert, R., Gros, N., SchulzeHessing, M., Kienast, B., and Rapp, M., 2011, "Biomechanical analysis of a synthetic femur spiral fracture model: Influence of different materials on the stiffness in flexible intramedullary nailing." Clin Biomech, 26 (6), 592-597.

[143] Flinck, M., von Heideken, J., Janarv, P. M., Wåtz, V., and Riad, J., 2014, "Biomechanical comparison of semi-rigid pediatric locking nail versus titanium elastic nails in a femur fracture model." J Child Orthop, 9 77-84.

[144] Green, J. K., Werner, F. W., Dhawan, R., Evans, P. J., Kelley, S., and Webster, D. A., 2005, "A biomechanical study on flexible intramedullary nails used to treat pediatric femoral fractures." J Orthop Res, 23 (6), 1315-1320.

[145] Heffernan, M. J., Gordon, J. E., Sabatini, C. S., Keeler, K. A., Lehmann, C. L., O'Donnell, J. C., Seehausen, D. A., Luhmann, S. J., and Arkader, A., 2014, "Treatment of femur fractures in 
young children: A multicenter comparison of flexible intramedullary nails to spica casting in young children aged 2 to 6 years." J Pediatr Orthoped, 125 (2), 126-129.

[146] Samiezadeh, S., Tavakkoli Avval, P., Fawaz, Z., and Bougherara, H., 2015, "An Effective Approach for Optimization of a Composite Intramedullary Nail for Treating Femoral Shaft Fractures." J Biomech Eng, 137 (12), 121001.

[147] ASTM D570-98, 2010. Standard Test Method for Water Absorption of Plastics, West Conshohocken, PA, 10.1520/D0570-98R10E01, www.astm.org.

[148] ASTM D695 - 10, 2010. Standard Test Method for Compressive Properties of Rigid Plastics, West Conshohocken, PA, 10.1520/D0695-10, www.astm.org.

[149] ASTM D3039/D3039M-14, 2008. Standard Test Method for Tensile Properties of Polymer Matrix Composite Materials, West Conshohocken, PA, 10.1520/D3039_D3039M-14, www.astm.org.

[150] ASTM E8/E8M-13a, 2013. Test Methods for Tension Testing of Metallic Materials, West Conshohocken, PA, 10.1520/E0008_E0008M-13A, www.astm.org.

[151] ASTM D7264/D7264M-07, 2015. Standard Test Method for Flexural Properties of Polymer Matrix Composite Materials, West Conshohocken, PA, 10.1520/D7264_D7264M-15, www.astm.org.

[152] ASTM E143-13, 2013. Standard Test Method for Shear Modulus at Room Temperature, West Conshohocken, PA, 10.1520/E0143-13, www.astm.org.

[153] ASTM D785-08, 2008. Standard Test Method for Rockwell Hardness of Plastics and Electrical Insulating Materials, West Conshohocken, PA, 10.1520/D0785-08, www.astm.org. [154] Montesano, J., Fawaz, Z., and Bougherara, H., 2013, "Use of infrared thermography to investigate the fatigue behavior of a carbon fiber reinforced polymer composite." Compos Struct, 97 76-83.

[155] La Rosa, G., and Risitano, A., 2000, "Thermographic methodology for rapid determination of the fatigue limit of materials and mechanical components." Int J Fatigue, 22 (1), 65-73. [156] Mahboob, Z. Damage accumulation in flax-fabric-reinforced composites: characterisation \& modelling 2014. Ryerson University, PhD Research Proposal, Toronto, Canada. 
[157] Montesano, J., Bougherara, H., and Fawaz, Z., 2014, "Application of infrared thermography for the characterization of damage in braided carbon fiber reinforced polymer matrix composites." Compos Part B-Eng, 60 137-143.

[158] Teoh, S. H., 2000, "Fatigue of biomaterials: a review." Int J Fatigue, 22 (10), 825-837. [159] Deligianni, D. D., Katsala, N., Ladas, S., Sotiropoulou, D., Amedee, J., and Missirlis, Y. F., 2001, "Effect of surface roughness of the titanium alloy Ti-6Al-4V on human bone marrow cell response and on protein adsorption." Biomaterials, 22 (11), 1241-1251.

[160] Ponsonnet, L., Reybier, K., Jaffrezic, N., Comte, V., Lagneau, C., Lissac, M., and Martelet, C., 2003, "Relationship between surface properties (roughness, wettability) of titanium and titanium alloys and cell behaviour." Mater Sci Eng C, 23 (4), 551-560.

[161] Zweymuller, K. A., Lintner, F. K., and Semlitsch, M. F., 1988, "Biologic fixation of a press-fit titanium hip joint endoprosthesis." Clin Orthop Relat Res, (235), 195-206.

[162] Egol, K. A., Chang, E. Y., Cvitkovic, J., Kummer, F. J., and Koval, K. J., 2004, "Mismatch of current intramedullary nails with the anterior bow of the femur." J Orthop Trauma, 18 (7), 410-415.

[163] Bergmann, G., Deuretzbacher, G., Heller, M., Graichen, F., Rohlmann, A., Strauss, J., and Duda, G. N., 2001, "Hip contact forces and gait patterns from routine activities." J Biomech, 34 (7), 859-871.

[164] Morlock, M., Schneider, E., Bluhm, A., Vollmer, M., Bergmann, G., Muller, V., and Honl, M., 2001, "Duration and frequency of every day activities in total hip patients." J Biomech, 34 (7), 873-881.

[165] Utvag, S. E., and Reikeras, O., 1998, "Effects of nail rigidity on fracture healing. Strength and mineralisation in rat femoral bone." Arch Orthop Trauma Surg, 118 (1-2), 7-13. 